\title{
Experimental and numerical studies on the proposed application of hollow electron beam collimation for the LHC at CERN
}

\author{
Vince Moens \\ vince.moens@epfl.ch \\ École Polytechnique Fédérale de Lausanne (EPFL)
}

Supervisor at Fermilab:

Dr. Giulio Stancari

Fermilab, Illinois, USA

Supervisor at CERN:

Dr. Stefano Redaelli

CERN, Geneva, Switzerland

Thesis Director at EPFL:

Prof. Dr. Leonid Rivkin

EPFL, Lausanne, Switzerland

Tuesday $10^{\text {th }}$ September, 2013
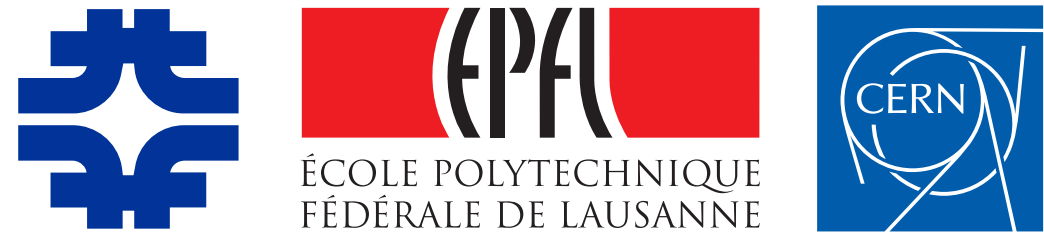


\section{Abstract}

This thesis work was carried out in the framework of the U.S. LHC Accelerator Research Program (USLARP), a collaboration between the European Organization for Nuclear Research (CERN) and the U.S. Department of Energy. The first half of the work was completed at Fermilab (USA), the location of the Tevatron, a proton-antiproton collider and the second largest particle collider in the world. The second half was completed at $C E R N$ (Switzerland), the location of the largest proton collider in the world (Large Hadron Collider ( $L H C$ )).

This thesis characterizes a Hollow Electron Beam (HEB) for possible usage at the $L H C$ to enhance its collimation through Hollow Electron Beam Lenses (HEBLs). Collimation is a long established principle in high energy particle accelerators. Hollow Electron Beam Collimation (HEBC) aims to enhance current collimation systems by controlling diffusion of primary halo particles into the limiting aperture. It works on the principle of a transverse radial electric field that kicks the primary halo particles outwards upon each pass in a multi-pass system. The transverse field is produced by a $H E B$ that is coaxially aligned with the accelerator beam, producing a negligible electric field in the center and a strong transverse electric field at amplitudes higher than the inner radius of the electron beam. Ideally, halo particles are affected without perturbation of the beam core. One of the main advantages of this system is to decrease the dependence on instantaneous loss spikes and beam jitter. A solid experimental basis of $H E B C$ was accumulated at the Tevatron. The application of this technique at the LHC is now under investigation.

The aim of this thesis is to present a preliminary report to support a future optimal conceptual design report. It characterizes the available hardware in order to facilitate the design of a Hollow Electron Gun (HEG) for the LHC, characterizes the effect on beam diffusion by determining the transverse electric fields of the electron beam and initiates 3D simulations in order to determine the effect of the $H E B L$ on the beam core. Experiments were conducted in the Tevatron Electron Lens Test Stand (TELTS) at Fermi National Accelerator Lab (Fermilab) (USA) in the lower Linear Accelerator (LINAC) gallery. 


\section{Acknowledgements}

Throughout my master thesis I have been able to count on the support of a number of professors, teaching assistants and fellow students.

First of all I would like to thank my thesis director Prof. Leonid Rivkin. It is due to his lecture on accelerator physics and his guidance throughout both of my semester thesis that I received the opportunity to complete my thesis at Fermilab. He has been an exceptional source of support and knowledge throughout all of my studies at the EPFL. Thank you for everything.

Secondly I would like to thank my supervisor at Fermilab, Dr. Giulio Stancari. He was a source of inspiration and knowledge for the experimental measurements and the theoretical deductions. His door was always open, whether it be for a friendly chat or a series of questions on the various aspects of Hollow Electron Beam Lenses (HEBLs). I am very grateful for everything he has done for me during my stay at Fermilab.

Thirdly I would like to thank my supervisor at CERN, Dr. Stefano Redaelli. He has been instrumental in organizing this master thesis and giving me valuable knowledge and insight into the operations of the LHC. He has acted as my supervisor for more than a year and I would like to thank him for everything I was able to learn from him.

Additionally I would like to thank Dr. Alexander Valishev, Dr. Moses Chung and Dr. Valentina Previtali. As fellow members of the team at Fermilab, they were always available in order to discuss aspects of the research or help me find my way around the new labs and new infrastructures. I would especially like to thank Dr. Moses Chung for his support on the WARP simulations.

The computer simulations on the Fermilab Accelerator Simulations Cluster would not have been possible without Dr. Eric Stern and Dr. Amitoj Singh. Furthermore I would like to thank Dr. David Grote from the Lawrence Berkeley National Laboratory. As one of the authors of WARP, he was an instrumental source of knowledge and technical support.

Of course all of this would not be possible without the support of ones family and I would thus like to thank my parents, Helena Van Swygenhoven and Jan Moens. Additionally I would like to thank Yasmin Al-Samarrai for her moral support. Lastly I would like to thank all my new friends at Fermilab and in Naperville for the awesome time we spent together. 


\section{Contents}

Acronyms

$\begin{array}{lll}\text { List of Figures } & \text { VII }\end{array}$

$\begin{array}{lll}\text { List of Tables } & \text { VIII }\end{array}$

1 Introduction $\quad 1$

2 Particle Accelerators and Collimation $\quad 3$

2.1 Accelerators and Particle Collimation . . . . . . . . . . . . . . . . . . 3

2.2 Beam Collimation Systems . . . . . . . . . . . . . . . . . . . . . . 4

2.2.1 Tevatron Collimation System . . . . . . . . . . . . . . . . . 4

2.2 .2 LHC Collimation System _ . . . . . . . . . . . . . . . . . . 5

2.3 Hollow Electron Beam Collimation $(\mathrm{HEBC}) \ldots \ldots \ldots \ldots$

2.3 .1 Tevatron experience for HEBC at LHC . . . . . . . . . . . . . . . . 8

3 Tevatron Electron Lenses and Guns $\quad 9$

3.1 Tevatron Electron Lenses _. . . . . . . . . . . . . . . . . . . . . . 9

3.1.1 Tevatron Electron Lens 2 Design Considerations _ . . . . . . . . . . . . . 9

3.1.2 Tevatron Electron Lens Test Stand Design Considerations . . . . . . . . . . . . . 10

3.1.3 Straight Hollow Electron Beam Lens Design . . . . . . . . . . . . . . . . . 10

3.2 Design of the Electron Guns . . . . . . . . . . . . . . . . . . . . . . 11

$3.2 .1 \quad 0.6$ Inch Hollow Electron Gun . . . . . . . . . . . . . . . . . . . . 11

3.2 .21 Inch Hollow Electron Gun . . . . . . . . . . . . . . . . . . . . 12

4 Thermionic Emission $\quad 15$

4.1 Temperature Limited Emission . . . . . . . . . . . . . . . . . . 15

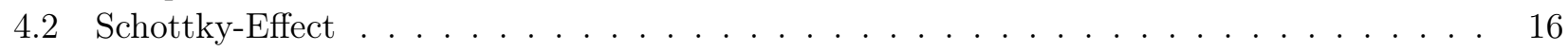

4.3 Space Charge Limited Emission . . . . . . . . . . . . . . . . . . . . . . 16

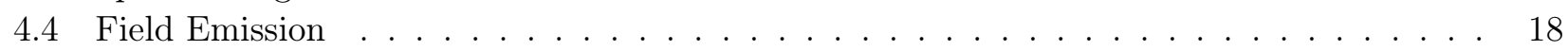

4.5 Summary of Thermionic Emission . . . . . . . . . . . . . . . . . . . . 19

51 Inch Hollow Electron Gun Characterization $\quad 20$

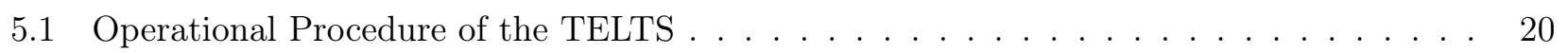

5.2 Transport Measurements . . . . . . . . . . . . . . . . . . . . 21

5.3 Yield Measurements . . . . . . . . . . . . . . . . . . . . . . . . 22

5.3 .1 Global Perveance . . . . . . . . . . . . . . . . . . . . . . . . . . . . . . . . 23

5.3 .2 Global Perveance from SAM Simulations . . . . . . . . . . . . . . . . . . . . 23

5.3 .3 Local Perveance . . . . . . . . . . . . . . . . . . . . . . . . 24

5.3.4 Dependence of Perveance on Filament Current . . . . . . . . . . . . . . . . 26

5.3.5 Generalized Perveance . . . . . . . . . . . . . . . . . . . . 28

5.4 Profile Measurements . . . . . . . . . . . . . . . . . . . . . . . . . . . . . . 29 
5.4 .1 Transverse Profile Measurement . . . . . . . . . . . . . . . . . . . . . . 29

5.4 .2 Beam Evolution . . . . . . . . . . . . . . . . . . . . 34

5.4 .3 Beam Scaling . . . . . . . . . . . . . . . . . . . . . . . 36

6 Transverse Fields of Measured Profiles $\quad 41$

6.1 Transverse Field of Hollow Electron Beams . . . . . . . . . . . . . . . . . . . . 41

6.2 Emittance Growth . . . . . . . . . . . . . . . . . . . . . . . . . 42

6.3 Method and Script . . . . . . . . . . . . . . . . . . . . . . . 43

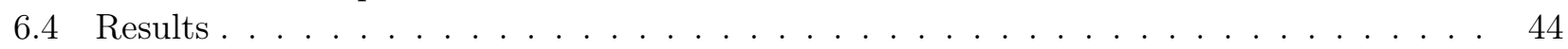

6.4 Field Measurements . . . . . . . . . . . . . . . . . . . . 44

6.4 .2 Upper Limit for Emittance growth . . . . . . . . . . . . . . . . . . . 45

7 Numerical Simulations of Electron Beam Dynamics $\quad 48$

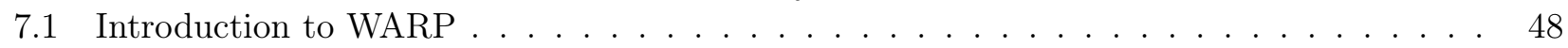

7.2 General Structure of the WARP Script . . . . . . . . . . . . . . . . . . . 49

7.3 Gun Injection . . . . . . . . . . . . . . . . . . . . . . . . . . . 51

7.4 Profile Injection . . . . . . . . . . . . . . . . . . . . . 53

7.5 Directions for further studies on 3D WARP simulations $\ldots \ldots \ldots \ldots$

8 Conclusion $\quad 56$

$\begin{array}{lc}\text { Works Cited } & 58\end{array}$

$\begin{array}{lr}\text { Appendices } & 61\end{array}$

Appendix A LHC and Tevatron Parameters $\quad$ A-1

Appendix B Measured Profiles $\quad$ B-1

B.1 List of measured profiles . . . . . . . . . . . . . . . . . B-1

Appendix C Codes $\quad$ C-1

C.1 Profile Measurement . . . . . . . . . . . . . . . . . . . . . . . . . C-1

C.2 Transverse Field Measurement . . . . . . . . . . . . . . . . . . . . . . . C-12

C.3 Numerical WARP simulations . . . . . . . . . . . . . . . . . . . . C-15

Appendix D Dimensions $\quad$ D-1

D.1 Electron Guns . . . . . . . . . . . . . . . . . . . . . . . . . D-1

D.2 Electron Lenses . . . . . . . . . . . . . . . . . . . . . . D-2

Appendix E Maps $\quad$ E-1

E.1 Map of LHC Collimators . . . . . . . . . . . . . . . . . . . . . E-1

E.2 Map of Tevatron . . . . . . . . . . . . . . . . . . . . . . E-2 


\section{Acronyms}

\begin{tabular}{|c|c|c|}
\hline Notation & Description & Page List \\
\hline ACL & Accelerator Command Language & $29-31$ \\
\hline ACNET & Fermilab Accelerator Control System & $20,29,30$ \\
\hline BNL & Brookhaven National Laboratory & 6 \\
\hline CERN & European Organization for Nuclear Research & $\mathrm{I}, 1,5,56,57$ \\
\hline CGM & Computer Graphics Metafile & 49 \\
\hline $\mathrm{COM}$ & Center Of Mass & 4 \\
\hline DTL & Drift Tube LINAC & 3 \\
\hline EGR & Emittance Growth Rate & 44,47 \\
\hline Fermilab & Fermi National Accelerator Lab & $\mathrm{I}, 1,4,9,12,23,48$ \\
\hline GNU & GNU's Not Unix & 31 \\
\hline HEB & Hollow Electron Beam & $\begin{array}{l}\mathrm{I}, 1,6,7,14,15,20,33,40 \\
41,47,55-57\end{array}$ \\
\hline HEBC & Hollow Electron Beam Collimation & $\mathrm{I}, 1,3,7-10,20,57$ \\
\hline HEBL & Hollow Electron Beam Lens & $\begin{array}{l}\text { I, II, } 1,2,7,8,11,18,20,29, \\
41,43,48,50,56,57\end{array}$ \\
\hline HEG & Hollow Electron Gun & $\mathrm{I}, 1,8,42,56,57$ \\
\hline HG06 & 0.6 inch Hollow Electron Gun & $1,8,9,11-14,22,56$ \\
\hline HG1b & 1 inch Hollow Electron Gun & $1,9,11-15,21,22,25,49,56$ \\
\hline HL-LHC & High Luminosity LHC & 5 \\
\hline IR & Interaction Region & 5,14 \\
\hline LHC & Large Hadron Collider & $\begin{array}{l}\mathrm{I}, 1,2,4,5,8,9,11,14,41 \\
55-57\end{array}$ \\
\hline LINAC & Linear Accelerator & $\mathrm{I}, 4$ \\
\hline $\mathrm{PIC}$ & Particle-In-Cell & $48,49,57$ \\
\hline RHIC & Relativistic Heavy Ion Collider & 6 \\
\hline RMS & Root Mean Square & $5,43,44$ \\
\hline SCLER & Space Charge Limited Emission Region & $15,16,18,19,23,24,26$ \\
\hline
\end{tabular}




\section{Notation Description}

TEL1 Tevatron Electron Lens 1

TEL2 Tevatron Electron Lens 2

TELTS Tevatron Electron Lens Test Stand

TEV Accelerator Simulations Cluster

TLER Temperature Limited Emission Region

US-LARP U.S. LHC Accelerator Research Program

VLHC Very Large Hadron Collider

\section{Page List}

$6,8,9$

$1,6,8,9,11,48,55$

I, 9-11, 20, 24, 29, 31, 43, 54, 56,57

$48,52,54$

$15-19,24,26$

I, 1

5 


\section{List of Figures}

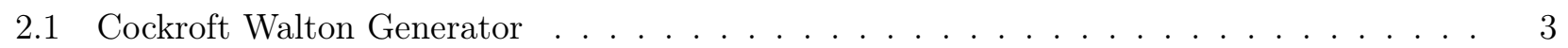

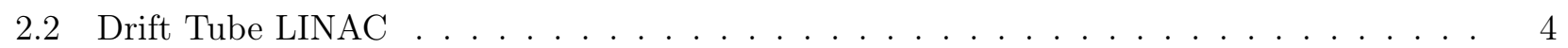

2.3 Ernest Lawrence and his cyclotron . . . . . . . . . . . . . . . . . . . . 4

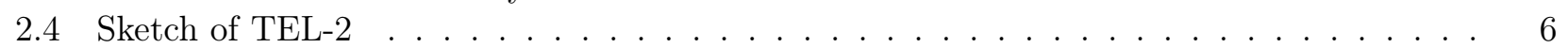

2.5 Radial electric field of Hollow Electron Beam . . . . . . . . . . . . . . . 7

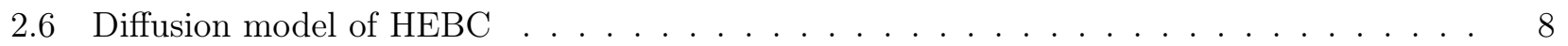

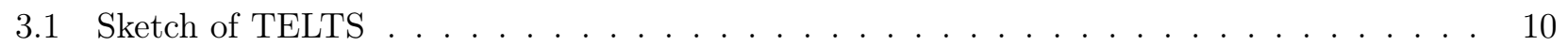

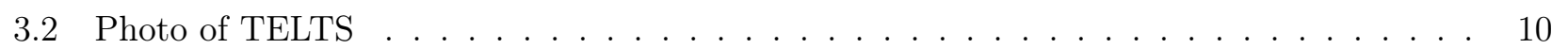

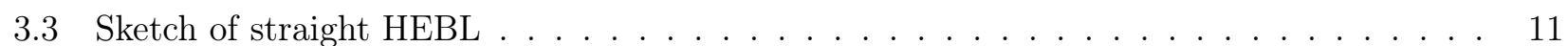

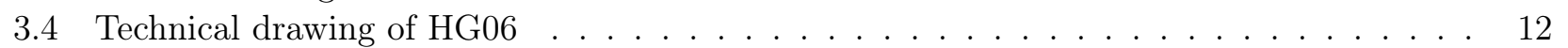

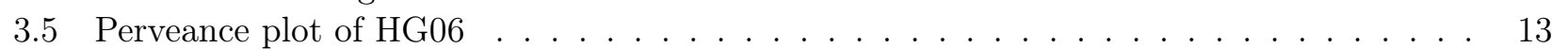

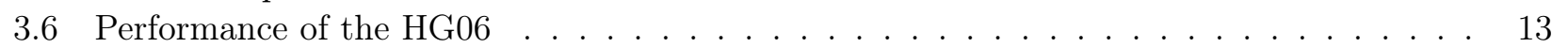

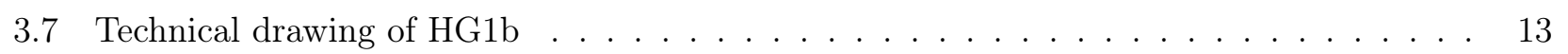

4.1 Energy levels in a cathode . . . . . . . . . . . . . . . . . . . 15

4.2 Space charge reduction of potential at cathode $\ldots \ldots \ldots \ldots \ldots$

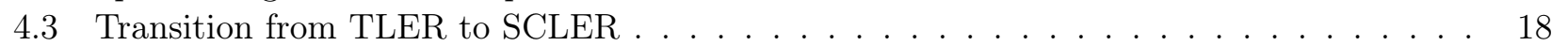

4.4 Field emission . . . . . . . . . . . . . . . . . . . . . . . . . . . . . . . 19

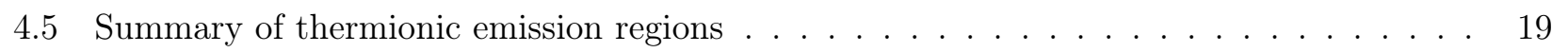

5.1 Cathode temperature vs. filament resistance and current . . . . . . . . . . . . . . . 21

5.2 Asymmetric profiles during May conditioning . . . . . . . . . . . . . . . . 22

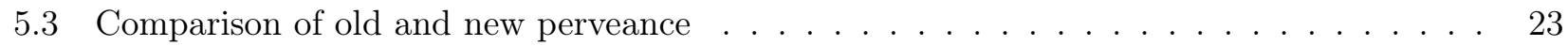

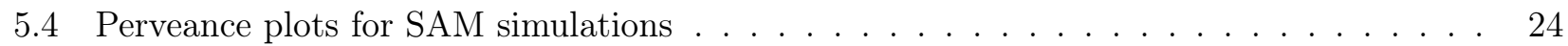

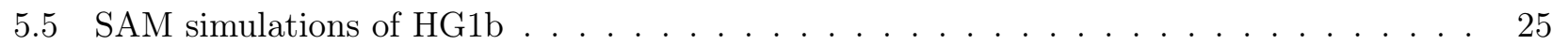

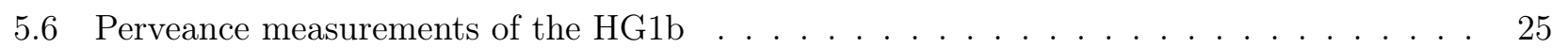

5.7 Number of ions formed per $\mathrm{cm}$ per mbar for various gases . . . . . . . . . . . . . 26

5.8 Temperature dependence of yield and perveance of HG1b f . . . . . . . . . . . . 27

5.9 Close up of temperature dependence of yield and perveance of HG1b . . . . . . . . . 28

5.10 Profiles measured after transport improvement . . . . . . . . . . . . . . . 32

5.11 Profiles measured before transport improvement . . . . . . . . . . . . . . . . 33

5.12 Transverse beam profile at $\mathrm{B}=1-4-1 \mathrm{kG}, \mathrm{V}=500 \mathrm{~V}$ and $\mathrm{I}_{\text {peak }}=73 \mathrm{~mA} \ldots \ldots . \ldots 34$

5.13 Transverse beam profile evolution at $\mathrm{B}=3.2 \mathrm{kG} \ldots \ldots \ldots \ldots$

5.14 Transverse beam profile evolution at $V=2 \mathrm{kV} \ldots \ldots \ldots \ldots \ldots \ldots$

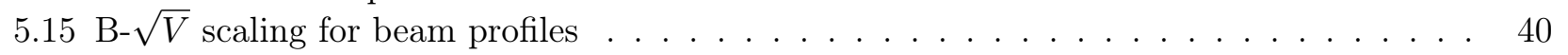

6.1 Charge density, electric potential and electric field at $\mathrm{B}=1-4-1 \mathrm{kG}$ and $\mathrm{V}=500 \mathrm{~V}$. . . . . 46

6.2 Charge density, electric potential and electric field at $\mathrm{B}=4 \mathrm{kG}$ and $\mathrm{V}=8 \mathrm{kV}$. . . . . . 47

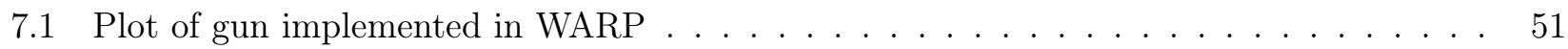

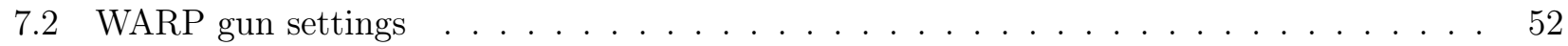


7.3 Electrostatic potential and fields in WARP gun . . . . . . . . . . . . . 52

7.4 Full electron beam in WARP from gun injection . . . . . . . . . . . . . . 53

7.5 Particle distribution of measured profile at $\mathrm{B}=1-4-1 \mathrm{kG}$ and $\mathrm{V}=500 \mathrm{~V} \ldots \ldots . \ldots 53$

7.6 Full electron beam in WARP from profile injection . . . . . . . . . . . . 54

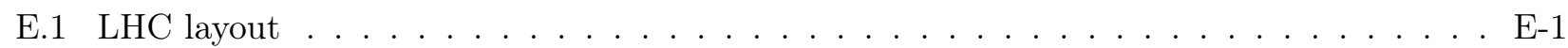

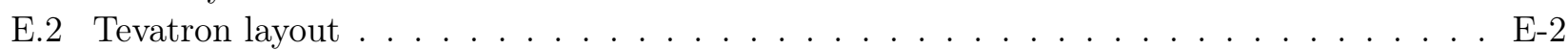

\section{List of Tables}

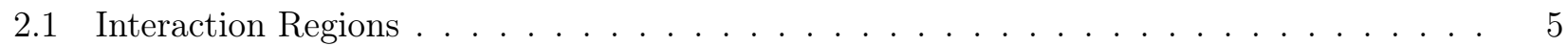

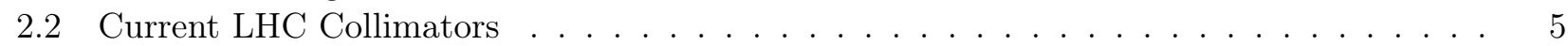

3.1 Design parameters for TEL $2 \ldots \ldots \ldots \ldots \ldots \ldots \ldots$

3.2 Design parameters for HG1b in a electron lens . . . . . . . . . . . . . . . . . 12

5.1 Pre and post transmission improvement solenoidal magnet settings . . . . . . . . . . . 22

5.2 Global perveance measurements . . . . . . . . . . . . . . . . . . . . . 23

5.3 SAM perveance . . . . . . . . . . . . . . . . . . . . . . . 24

5.4 Variables for transverse profiles . . . . . . . . . . . . . . . . . . . . . . 29

5.5 Corrector settings in beam tube and $\mathrm{COM}$ of profiles . . . . . . . . . . . . 30

5.6 Rotation angles of particles halfway between outer and inner cathode radius . . . . . . 36

5.7 Physical quantities for dimensional analysis of $B-\sqrt{V}$ scaling law . . . . . . . . . . 39

6.1 LHC emission growth rates . . . . . . . . . . . . . . . . . . . . 45

6.2 Transverse profile induced emittance growth rates . . . . . . . . . . . . . . . 45

A.1 Important parameters of Tevatron and LHC . . . . . . . . . . . . . . . A-1

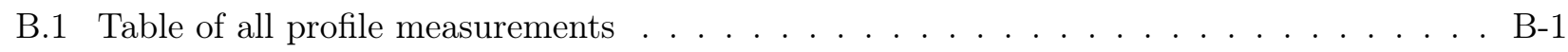




\section{Chapter 1}

\section{Introduction}

This work was completed in the framework of the U.S. Department of Energy collaboration with the Large Hadron Collider (LHC), the U.S. LHC Accelerator Research Program (US-LARP). The experiments were completed between February and May 2013 at Fermi National Accelerator Lab (Fermilab) in Illinois, USA. Simulations were done at the European Organization for Nuclear Research (CERN) in Switzerland in June and July 2013. The work incorporates aspects of the Tevatron, a proton and antiproton collider, and the LHC, a proton-proton collider.

This thesis addresses the characterization of a new 1 inch Hollow Electron Gun (HG1b) tested at Fermilab for $L H C$ collimation purposes in the framework of Hollow Electron Beam Collimation (HEBC). Considering successful soft collimation experiments using a 0.6 inch Hollow Electron Gun (HG06) in the Tevatron Electron Lens 2 (TEL2) at the Tevatron, this thesis outlines important design parameters for a Hollow Electron Gun (HEG) and an electron lens for the LHC. It measures the transmission, yield and transverse profiles of the new $H G 1 b$, which fits the general $L H C$ requirements. Furthermore it determines the transverse electric fields and the beam evolution of the Hollow Electron Beam (HEB). Additionally upper limits for the emittance growth of the proton core due to the $H E B$ are determined.

The first Tevatron electron lens was installed in 2001. It was used on a daily basis for abort gap cleaning for a period of 10 years until the recent shutdown of the Tevatron in 2011. A second Tevatron electron lens was installed in 2006 as a backup for the first lens. It was mainly used for research on beambeam force compensation and space charge compensation [1]. More recently, the lens has been tested as a soft collimator by installing a $H E G$. Considering the 10 years of prolonged stable operation at the Tevatron, electron lenses have established themselves as a mature technology for beam manipulation.

$H E B C$ is designed to be used together with current collimation systems in order to control the diffusion of halo particles into the limiting aperture through transverse electric fields. The proton core at the same time remains unaffected due to the negligible electric field that is found in a cylindrical hollow electron beam.

$H E B C$ studies have been part of US-LARP since 2009. There is a strong interest to implement two electron lenses in the $L H C$. The aim is to provide a solid basis for the design of those lenses, in case these are needed when the $L H C$ reaches nominal energy. The TEL2 is now available for use at the $L H C$, but constraints such as cryogenics and a long installation time make this a difficult task. Therefore the plan is to design two new electron lenses, optimized specifically for operation at $7 \mathrm{TeV}$, with improved integration possibilities and instrumentation [2, S.27-28].

The CERN strategy for the Hollow Electron Beam Lens (HEBL) was presented at the 20th USLARP collaboration meeting in Napa Valley, CA, USA, in April 2013, it was agreed that Fermilab would work on an optimum conceptual design for an implementation in the LHC. This study would entail a discussion of the hardware parameters and optimal design parameters for the LHC, the effect of the $H E B L$ on beam dynamics and beam core (luminosity) and the enhancement of collimation performance. The effect on beam dynamics was addressed in [3]. This recent paper shows that with an AC beam mode operation, a scraping of $75 \%$ of the halo particles was possible in $20 \mathrm{~s}[3]$.

This thesis will address the hardware and optimal design parameters as well as the effects of $H E B C$ 
on beam dynamics and the beam core. This is done by discussing current Tevatron hardware, measuring the yield and transverse profiles of the electron beam and determining the transverse electric fields of the electron beam. Additionally this thesis initiates a 3D simulation using WARP which will be used in order to obtain a 3D non-linear kick model for implementation in Lifetrack or SixTrack. This will allow the integration of proton trajectories in the $L H C$ passing through the $H E B L$ and thus a analysis of the effect of the electron lens on beam halo diffusion and on the beam core. An overarching aim of this thesis is to present a first study that will support a future optimal conceptual design report. 


\section{Chapter 2}

\section{Particle Accelerators and Collimation}

This chapter introduces particle collimation as a necessity for current high energy particle accelerators. It discusses the limitations of classical collimation systems and elaborates on Hollow Electron Beam Collimation (HEBC).

\subsection{Accelerators and Particle Collimation}

The origin of particle accelerators can be traced back to Rutherford's 1910 scattering experiment of $\alpha$ particles on gold foil at Cavendish at Cambridge University in England. In 1927, Ernest Rutherford addressed the Royal Society of London, expressing his desire for the development of a device that could continuously produce $\alpha$ and $\beta$ particles at energies much larger than their natural decay energies. His goal was to disintegrate nuclei with binding energies that exceed that of nitrogen [4]. In 1930, the first particle accelerator was conceived by John D. Cockroft and E.T.S Walton at the laboratory [5]. Since it was yet impossible to accelerate particles such as $\alpha$ particles to energies beyond their radioactive equivalents, Cockroft and Walton decided to work with lighter particles, accelerating protons through a linear discharge tube with a potential of $200 \mathrm{kV}$. Lacking results with such "low energy" particles, they conceived a voltage multiplier, called the Cockroft-Walton generator (See fig. 2.1), allowing them to accelerate protons to $800 \mathrm{keV}$, which subsequently allowed them to force the decay of Lithium into two $\alpha$ particles, using protons

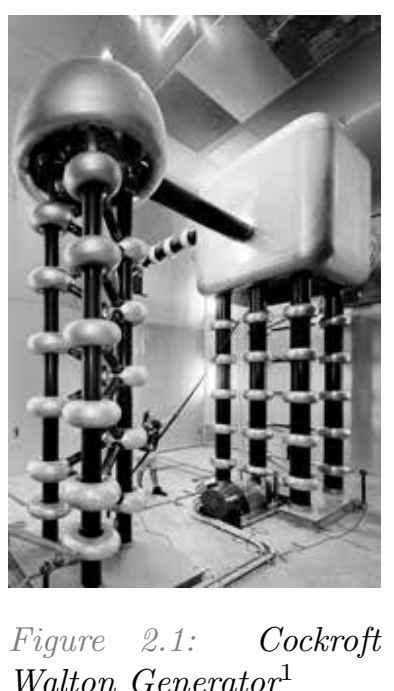
at $500 \mathrm{keV}$.

In 1931, Van der Graaf and Wideröe followed suit. Especially Rolf Wideröe's design, the Drift Tube LINAC (DTL), was a cornerstone for the development of today's particle accelerators. It accelerated particles through axially aligned drift tubes, separated by strong electromagnetic fields. By applying an AC current, he could tune the frequency of the field, such that the particles would be boosted upon every exit of a drift tube (see fig. 2.2). This design removed the need to store huge electric charges, which limited Cockroft and Van der Graaf's designs. While Wideröe's design was limited to around $50 \mathrm{keV}$ of energy, it was his design that inspired Ernest Lawrence to develop the cyclotron, a device that is widely known as the forerunner of particle accelerators (see fig. 2.3). In effect, the cyclotron is equivalent to winding Wideröe's design around and around itself to create a magnetically confined beam orbit with an alternating electric field.

In recent years, the spectrum of different applications of particle accelerators has widened. Leadingedge accelerators are driven by particle physics requirements (beam energy, high luminosity and intensity), but the majority of operating accelerators serve other purposes such as chemical or material research and medical treatments. For a history on particle accelerators, see [6].

\footnotetext{
${ }^{1}$ http://www.visualphotos.com/photo/1x6033020/cockroft-walton_generator_fermilab_a090057.jpg

${ }^{2}$ http://www.fnal.gov/pub/science/experiments/energy/images/95-1039-small.jpg
} 
Today's state of the art accelerators reach energies that are approximately $10^{5}$ times higher. The highest to date recorded acceleration energy was observed at the Large Hadron Collider ( $L H C$ ) with a value of $4 \mathrm{TeV}$, giving $8 \mathrm{TeV}$ in the Center Of Mass (COM) frame. It is designed to hold a stored energy of $\approx 360 \mathrm{MJ}$ for each beam [7]. Such energies require the use of superconducting magnets to steer the beam, resulting in the need for strong collimation. Even under good stable operational conditions, particles will leave the optimal design path of the accelerator due to beam-gas interactions, intra-beam scattering, beam-beam interactions, RF noises, ground noises and resonances due to imperfections in the accelerator elements [8]. The main process of intrabeam interactions results in a slow diffusion of protons from the axial path and a subsequent growth of the emittance. These particles together form the halo, a set of off-axis particles whose betatron amplitudes increase gradually until they are caught by collimators or other limiting apertures.

The beta function is the envelope around all particle trajectories in the accelerator. The betatron amplitude is the transverse amplitude with respect to the nominal beam orbit of individual particles in the accelerator. The collision of particles with the limiting aperture causes hadronic and electromagnetic showers, which can negatively affect accelerator components and detectors with different severities.

Collimation refers to the spatial alignment and the reduction of the spatial cross-section of a beam halo. It is the removal of the beam halo, in order to protect components against excessive irradiation, prevent quenches in the superconductors, minimize background noise in the detectors and reduce the radiation on personnel and the environment [9, 10, p. 3, p. 297]. Collimators can act as diagnostic tool for accelerator admittances, beam vibrations and

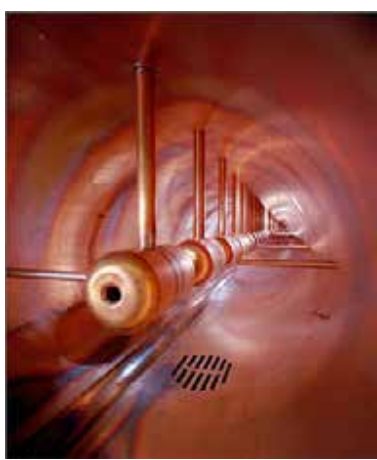

Figure 2.2: Drift Tubes in the LINAC facility at Fermilab ${ }^{2}$

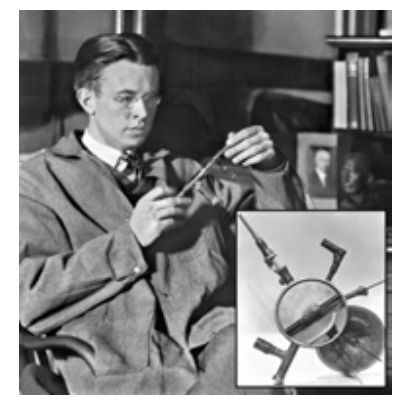

Figure 2.3: Ernest Lawrence with his cyclotron design ${ }^{3}$ diffusion rates [11].

\subsection{Beam Collimation Systems}

Collimation of beam halo particles is achieved by limiting the physical aperture of the accelerator beam using solid blocks of absorbing material. Through scattering, a small fraction of halo particles can escape these blocks. We call such particles out-scattered particles. The cleaning inefficiency, the leakage of halo protons to sensitive equipment, of the collimation system is improved by using several collimation stages. Such a system consists of primary collimators, whose purpose is to intercept the primary beam halo through multiple Coulomb scattering. Secondary collimators are used to intercept the secondary halo particles which are out-scattered from the primary collimators at higher amplitudes [12]. The LHC uses a complex four stage collimation system. A list of the current $L H C$ collimators is given in tab. 2.2.

\subsubsection{Tevatron Collimation System}

Tevatron had two Collider Runs. Inbetween the two runs, the collimation system of the Tevatron was upgraded [9]. During Collider Run I, the Tevatron used a single stage collimation system that consisted of $1 \mathrm{~m}$ long solid absorbers which allowed the Fermi National Accelerator Lab (Fermilab) to raise the Tevatron efficiency of the fast resonant extraction system by a factor 5 [13].Using this system at Tevatron, one obtained a low cleaning efficiency close to 0.5 [13]. This system was insufficient and caused operational limitations, such that in Collider Run II, a two-stage automated collimation system was implemented [9]. At Tevatron collimation is a discrete process in which the beam halo is regularly removed. The new design required that the entire halo removal could be conducted in approximately $5 \mathrm{~min}$. The system incorporated four primary collimators (targets) and eight new $1.5 \mathrm{~m}$ long secondary

\footnotetext{
${ }^{3}$ http://newscenter. lbl.gov/wp-content/uploads/lawrence-cyclotron.jpg
} 


\begin{tabular}{|l|l||l|}
\hline $\begin{array}{l}\text { Interaction } \\
\text { Regions }\end{array}$ & $\begin{array}{l}\text { Purpose } \\
\text { Purpose }\end{array}$ & $\begin{array}{l}\text { Collimator } \\
\text { Types }\end{array}$ \\
\hline \hline IR1 & ATLAS experiment & TCT, TCL \\
IR2 & $\begin{array}{l}\text { ALICE experiment } \\
\text { \& Injection Beam 1 } \\
\text { TCLI, TCT, TDI }\end{array}$ & \\
IR3 & Momentum cleaning & TCP, TCSG, TCLA \\
IR4 & RF cavities & - \\
IR5 & CMS experiment & TCT, TCL \\
IR6 & Beam Dump & TCSG, TCDQA \\
IR7 & Betatron Cleaning & TCP, TCSG, TCLA \\
IR8 & LHC-b experiment & TCLI, TCT, TDI \\
& \& Injection Beam 2 & \\
\hline
\end{tabular}

Table 2.1: The 8 interaction regions, their purpose and collimator types.

\begin{tabular}{|l|c|c|c|c|}
\hline \hline Functional type & Name & Plane & Num. & Material \\
\hline \hline Primary IR3 & TCP & $\mathrm{H}$ & 2 & CFC \\
Secondary IR3 & TCSG & $\mathrm{H}$ & 8 & CFC \\
Absorbers IR3 & TCLA & $\mathrm{H}, \mathrm{V}$ & 8 & W \\
Primary IR7 & TCP & H,V,S & 6 & CFC \\
Secondary IR7 & TCSG & H,V,S & 22 & CFC \\
Absorbers IR7 & TCLA & H,V & 10 & W \\
Tertiary IR1/2/5/8 & TCT & H,V & 16 & W/Cu \\
Physics debris absor. & TCL & H & 4 & Cu \\
Dump protection & TCSG & H & 2 & CFC \\
& TCDQ & H & 2 & C \\
Inj. prot. (lines) & TCDI & H,V & 13 & CFC \\
Inj. prot. (ring) & TDI & V & 2 & C \\
& TCLI & V & 4 & CFC \\
& TCDD & V & 1 & CFC \\
\hline \hline
\end{tabular}

Table 2.2: Current LHC Collimators. For a complete list of Acronyms, visit the Acronym website of the $L H C^{4}$.

collimators. The primary collimators are $5 \mathrm{~mm}$ tungsten plates, placed at $5 \sigma$. $\sigma$ is the standard deviation of the Gaussian distribution of the accelerator beam. They create a Root Mean Square (RMS) transverse kick of $17 \mu \mathrm{rad}$ at $980 \mathrm{GeV}$ [11]. The secondary collimators are $1.5 \mathrm{~mm}$ steel blocks placed at $6 \sigma$ [9]. Additionally a new control system was implemented that would allow the fast processing of beam loss monitor data and a fast beam intensity feedback control, in which the full travel of the collimator took $15 \mathrm{sec}$ [9]. Next to this major upgrade, the system was upgraded on a regular basis, including the installation of a tertiary collimator in 2003.

Measurements have shown that the cleaning inefficiency was 0.001 . At the Tevatron collimation is primarily used for background reduction in physics, which was of the order of $6.7 \times 10^{-3}$, defined as the ratio of background noise with and without collimation $[9$, p. 7]. The estimated evolution of beam losses due to $\bar{p} p$ collisions, losses in the RF-bucket and beam-gas scattering are reviewed in [13, p. 14].

\subsubsection{LHC Collimation System}

Particle accelerators such as the $L H C$, with a nominal stored energy of $360 \mathrm{MJ}$ require more collimation systems. At the $L H C$, the beam is continually cleaned. Even small losses in the $L H C$ of the order of $4 \times 10^{7}$ protons, could cause quenches in the superconducting magnets through a deposition of energy in the order of $30 \mathrm{~mJ} \mathrm{~cm}^{-3}$ [14].

The beam cleaning system at the LHC requires a cleaning efficiency of $99.998 \%$. The $L H C$ has two cleaning regions in Interaction Region (IR)3 and IR7 and several additional collimators in the other sections, with a total of 100 collimators, absorbers and similar devices [7]. There are 8 IRs in the LHC with different purposes. The IRs and their purposes are listed in table 2.1. The cleaning insertions have primary (TCP), secondary (TCS) and absorber (TCLA) collimators. Tertiary collimators (horizontal TCTH and vertical TCTV) are placed upstream of the experiments in IR1, IR2, IR5, and IR8. The machine is protected in IR6 against beam dump failures through dump protection devices (TCS6 and TCDQ) [7].

\subsection{Hollow Electron Beam Collimation (HEBC)}

Considering projects such as the High Luminosity LHC (HL-LHC) at European Organization for Nuclear Research (CERN), which aims at increasing the luminosity of the $L H C$ by a factor 10, or the Very Large Hadron Collider (VLHC), designed to operate at $20 \times 20 \mathrm{TeV}$ in Stage 1 and $88 \times 88 \mathrm{TeV}$ in Stage 2 [15], new innovative collimation techniques are required.

Some of the limitations of classical collimators is that they can not operate arbitrarily close to the beam orbit, due to impedance effects and instantaneous loss rates [11]. Additionally, beam loss rates

\footnotetext{
${ }^{4}$ http://Ihccwg.web.cern.ch/lhccwg/Bibliography/UsefulAcronyms.htm
} 

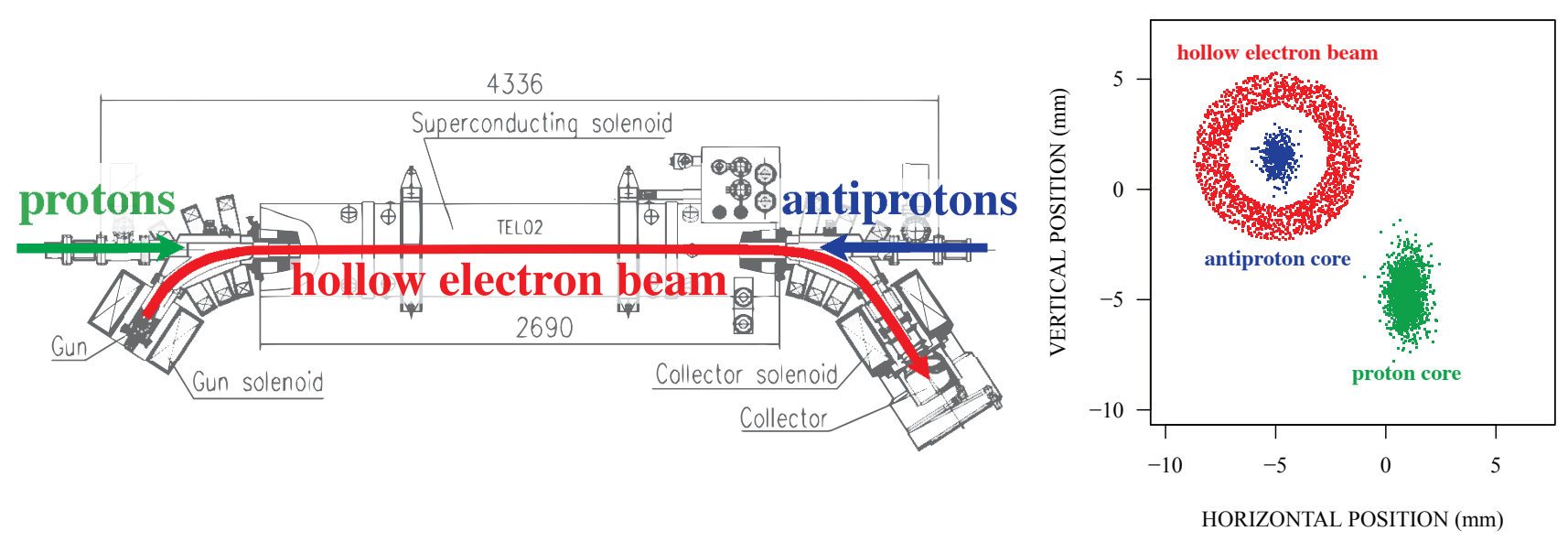

Figure 2.4: Technical Drawing of TEL2 Setup. Source: [8]

increase dramatically when moving collimator beam jaws inwards. Furthermore, classical collimators are affected by beam jitter, oscillations of the accelerator due to mechanical noise or ground motion [8]. Even with active stabilization, periodic bursts at the beam aperture are caused.

Electron lenses employ transverse fields of electron beams to interact with high energy bunches. An electron gun is used to produce an electron beam that is steered into the accelerator beam tube. It is aligned with the proton beam orbit, overlapping over a given length $L_{e}$, after which it is extracted from the accelerator beam tube into a collector. The electron beam is magnetically confined through solenoids. The electron gun and therefore the beam can be pulsed. All current electron lenses have the electron gun and the collector positioned outside the accelerator beam tube. A sketch of the Tevatron Electron Lens 2 (TEL2) is given in fig. 2.4.

An electron lens can produce different transverse profiles. For example, a Gaussian profile was used for non-linear beam-beam force compensation in the Tevatron and a flat-top profile was used for bunch by bunch tune correction. Further profiles are explained in [16]. An important advantage of the electron lens is that the electron beam current can be adjusted between individual bunches at the Tevatron, allowing single bunch manipulations. A normal figure of merit for electron lenses with Gaussian profiles is the shift of the accelerator beam tune due to the lens. A perfectly steered round electron beam, shifts the betatron tune by [17]

$$
\mathrm{d} Q_{x, y}= \pm \frac{\beta_{x, y} L_{e} r_{p}}{2 \gamma e c} j_{e}\left(\frac{1 \mp \beta_{e}}{\beta_{e}}\right)
$$

where $\beta_{e}=v / c$ is the electron beam velocity, $\gamma$ the Lorentz factor and $r_{p}=e^{2} / m c^{2}=1.53 \times 10^{-16}$ is the classical proton radius. The plus sign represents focusing for protons and defocussing for anti-protons.

There are two electron lenses installed in the Tevatron, Tevatron Electron Lens 1 (TEL1) and TEL2. Both operate around $10 \mathrm{keV}$ and at a couple of amperes. They can produce a tune shift of $\mathrm{d} Q_{x, y}^{\max } \approx 0.008$ [16]. They were installed for research on beam-beam compensation [17, 16]. TEL1 was installed in 2001. It has since been used in normal operation procedure for abort gap cleaning [18]. TEL2 was installed in 2006 as a backup for TEL1. Beam-beam compensation was observed, but was not needed after the installation of electron cooling for antiprotons in the recycler ring. Due to the reliability of TEL1, TEL2 was rarely needed for normal operations and thus used for further experiments on beam-beam compensation with different electron guns $[19,11]$. Electron lenses for beam-beam compensation are currently being comissioned at the Relativistic Heavy Ion Collider (RHIC) at Brookhaven National Laboratory (BNL) [20].

With an appropriate gun design, Hollow Electron Beams (HEBs) can be produced. Such a beam can be used for enhacning collimation. The $H E B$ is aligned coaxially with the proton beam, such that the proton beam core travels through the negligible electric field in the hollow space of the $H E B$. Halo particles of the proton beam at transverse amplitudes bigger than the inner radius $R_{i}$ experience near- 


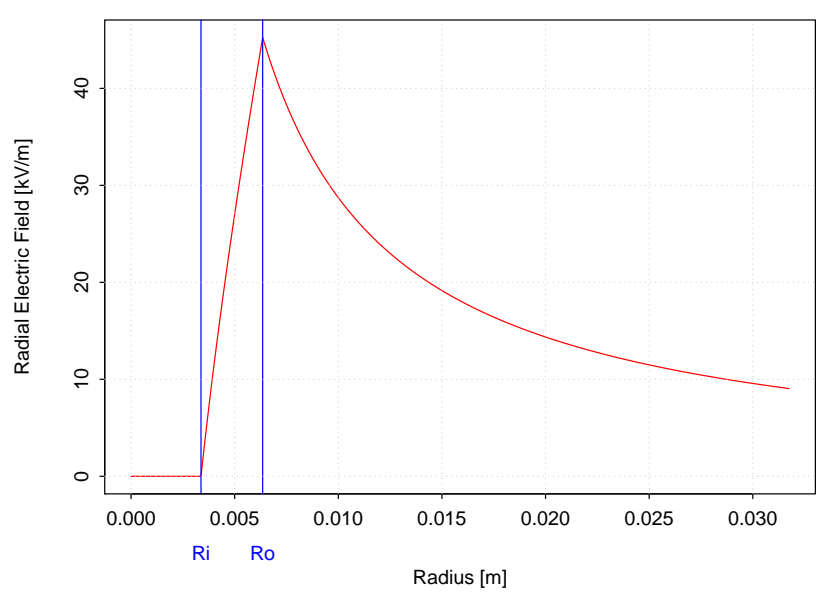

(a) $B=4 T$ and $V=500 \mathrm{~V}$

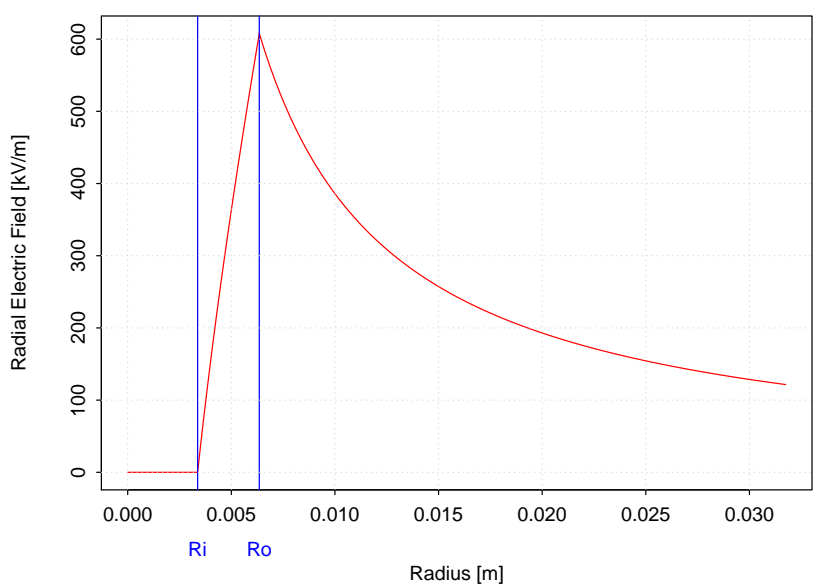

(b) $B=4 T$ and $V=8 k V$

Figure 2.5: Radial electric field for profiles corresponding to $V=500 \mathrm{~V}$ and $8 \mathrm{kV}, I=0.073 \mathrm{~A}$ and $3.88 \mathrm{~A}, R_{i}=3.4 \mathrm{~mm}$ and $R_{o}=6.4 \mathrm{~mm}$.

linear transverse radial kicks up to the outer radius $R_{o}$. Particles above $R_{o}$ experience a non-linear decay of the transverse kicks with respect to amplitude due to the Biot-Savart decay of the transverse electric field of the $H E B$ above its outer radius. A typical electric field profile is shown in fig. 2.5. Its derivation is discussed in chap. 6.

Assuming a cylindrical symmetry of the current density profile, transverse kicks are very controllable and given by [11]:

$$
\Theta=\frac{1}{4 \pi \varepsilon_{0}} \frac{2 I_{r} L\left(1 \pm \beta_{e} \beta_{p}\right)}{r \beta_{e} \beta_{p} c^{2}(B \rho)_{p}}
$$

where $\beta_{e} c$ is the electron velocity, $\beta_{p} c$ is the particle velocity, $I_{r}$ is the enclosed electron current, $L$ is the interaction length of theHollow Electron Beam Lens (HEBL)and $r$ is the distance from the axis of the beam. The + applies when the magnetic and electric forces have the same direction. For typical Tevatron parameters, the transverse kick given to $980 \mathrm{GeV}$ particles in Tevatron is $0.2 \mu \mathrm{rad}$.

The magnetic fields in the gun, collector and main solenoid can be varied individually. This allows the operator to compress the $H E B$ in the main solenoid. The compression follows the scaling law $\sqrt{B_{\text {main }} / B_{\text {gun }}}$ and the decompression law $\sqrt{B_{\text {coll }} / B_{\text {main }}}$. The magnetic field in each solenoid has an upper limit of $6 \mathrm{~T}$, which is easily attainable by superconducting magnets. It has a lower limit of $\approx 0.1 \mathrm{~T}$ due to scalloping of the beam. Scalloping refers to transverse oscillations in the beam due to transverse electric fields. Scalloping is reduced by magnetically confining the particles stronger. When increasing the $H E B$ size, it becomes necessary to compress the beams. This is shown in sec. 5.2.

The basic concept of the $H E B C$ is that $R_{i}$ is chosen to be smaller than the half gap of the primary collimators (TCP). It then causes the diffusion of particles into the limiting transverse aperture between $R_{i}$ and the transverse aperture. A sketch is shown in fig. 2.6. The proton beam is represented through the blue curve with long tails representing the halo. The diffusion coefficient is represented by the green curve. $H E B C$ allows the cleaning of the long tails of the transverse proton beam distribution. This makes the system less dependent on loss spikes such as those caused by beam jitter [8]. It is important to note that $H E B C$ requires that the current collimation system is kept in place.

Various effects can reduce the effectiveness of $H E B C$. Important requirements are: a sufficient beam current to produce significant transverse kicks, a negligible electric field in the center to reduce emittance growth and a symmetric beam distribution. Beam current should be above $1 \mathrm{~A}$, as was seen from experiments at the Tevatron [11]. The electric field is strongly affected by radial symmetry of the $H E B$. Furthermore bends in the electron lenses can adversely affect the electron beam distribution and the proton core as it pierces through the $H E B$ wall in the bend (see fig. 2.4.) The effectiveness of $H E B C$ depends furthermore on the time structures of the pulsed electron beam. Valentina Previtali recently 

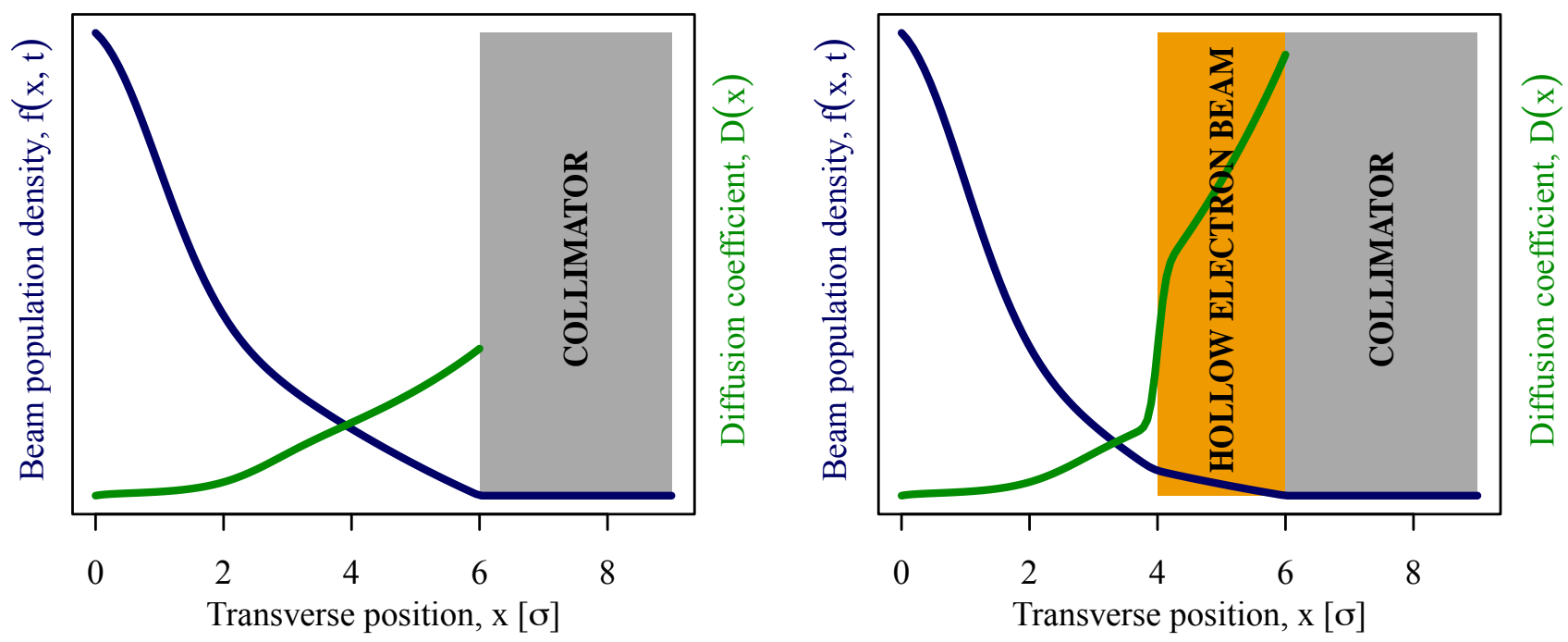

Figure 2.6: Schematic representation of the effect of the HEBC on the diffusion model of collimation. Source: [8].

completed a study of 3 different time structures; DC current, AC current and a "diffusive" current. These are possible, because the cathode has a rise-time short enough to allow turn by turn modulation in the $L H C$. Her studies show that the AC beam mode is the most efficient with a scraping of $75 \%$ of halo particles in less than $20 \mathrm{~s}$. For more details refer to [21].

\subsubsection{Tevatron experience for $\mathrm{HEBC}$ at $\mathrm{LHC}$}

Tevatron experiments from October 2010 to September 2011 gave an experimental foundation for the $H E B C$ concept in the context of beam studies for the LHC collimation purposes. They were done using a 0.6 inch Hollow Electron Gun (HG06) in the TEL2. The experiments showed that HEBLs are compatible with collider operations and that the alignment of the electron and proton beam is reliable and reproducible. It could be shown that the effects on the beam core were negligible, while a smooth halo removal was observed. Furthermore the loss-rate fluctuations due to beam jitter were reduced. Overall a transverse diffusion of the beam halo was observed. These achievements of the TEL2 at the Tevatron are explained in more detail in [11, 22, 8]. After 10 years of stable Tevatron electron lens operation in the Tevatron (TEL1 and TEL2), it is clear that the technology is reliable and thus ready for operation in the LHC. The $H E B L s$ which were used for $H E B C$ and its characterization are discussed in sec. 3.1. Furthermore a conceptual design of a $H E B L$ where the Hollow Electron Gun (HEG) and the collector are coaxially aligned with the accelerator beam is discussed. HEGs are an important part of the HEBLs and are discussed in sec. 3.2. 


\section{Chapter 3}

\section{Tevatron Electron Lenses and Guns}

The Tevatron Electron Lens 2 (TEL2), installed in 2006, was used as a backup for Tevatron Electron Lens 1 (TEL1) for abort gap cleaning and for studies on beam-beam compensation and Hollow Electron Beam Collimation (HEBC). The HEBC studies at the Tevatron were done using a 0.6 inch Hollow Electron Gun (HG06). Since 2012 a new 1 inch Hollow Electron Gun (HG1b) has been used in the Tevatron Electron Lens Test Stand (TELTS), which is used for yield and transverse profile measurements. It was designed specifically for the Large Hadron Collider ( $L H C$ ). This chapter discusses the electron lenses and electron guns that have been used for the development of HEBC at Fermi National Accelerator Lab (Fermilab) in the context of collimation studies for the LHC. It furthermore outlines design parameters for the $H E B C$ studies at Tevatron and proposes design parameters for the use of the $H G 1 b$ at the LHC at CERN.

\subsection{Tevatron Electron Lenses}

\subsubsection{Tevatron Electron Lens 2 Design Considerations}

The Tevatron Electron Lens 2 (TEL2) is one of two electron lenses installed in the Tevatron. It was used for beam-beam force compensation and for experimentation with $H E B C$. A sketch of the TEL2 is shown in fig. 2.4. Unlike the LHC, the Tevatron circulates a proton and an antiproton beam in opposite directions in the same vacuum pipe. An electron gun, situated left next to the accelerator beam tube, produces an electron beam that is sent on an arc through 3 short solenoids into the accelerator beam tube. After traveling a

\begin{tabular}{|l|l|}
\hline \hline$I_{\max } \pm(\Delta I / I)_{\max }$ & $1.2 \mathrm{~A} \pm 2 \%$ \\
$V_{\max }$ & $5 \mathrm{kV}$ \\
$R_{1} / R_{2}$ & $4.5 \mathrm{~mm} / 7.5 \mathrm{~mm}=0.6$ \\
$R_{1 \min }$ & $0.58 \mathrm{~mm}$ \\
$\Delta t_{\min }$ & $250 \mathrm{~ns}$ \\
\hline \hline
\end{tabular}

Table 3.1: Design parameters of TEL2 in Tevatron with HG06: maximum electron beam current $I_{\max }$ and current jitter $(\Delta I / I)_{\max }$, maximum extraction voltage $V_{\max }$, ratio between inner and outer cathode radius $R_{1} / R_{2}$, minimum achievable inner radius for the electron beam $R_{1 \text { min }}$ and cathode modulator rising time $\Delta t_{\min }$ [21] distance of $2 \mathrm{~m}$, during which the electron beam interacts with the (anti-)proton beam, the electrons are again extracted from the accelerator beam tube using 3 more solenoids [16]. The lens operates with a few amperes and at an energy up to $10 \mathrm{keV}$ [16]. The gun, the interaction region and the collector have 3 separate solenoids, used for the magnetic confinement of the electron beam. Their magnetic field strengths are designated $B_{\text {gun }}, B_{\text {main }}$ and $B_{\text {coll }}$ respectively. Inside the main solenoid, 6 superconducting dipole correctors are found. They are used to align the beam with the (anti-)proton beam. This requires five degrees of freedom, three for the axial position of the electron beam upstream of the main solenoid and two for its angle.

Since the shutdown of the Tevatron at Fermilab, the TEL2's hardware has become available for use. Its efficiency as a scraper was proven through extended tests at the Tevatron collider, driving the halo of a $980 \mathrm{GeV}$ anti-proton beam onto the collimators[23]. The TEL2 (using the HG06) was used for $H E B C$ at currents up to 1.2 A. TEL2 parameters used in the Tevatron are given in tab. 3.1. 


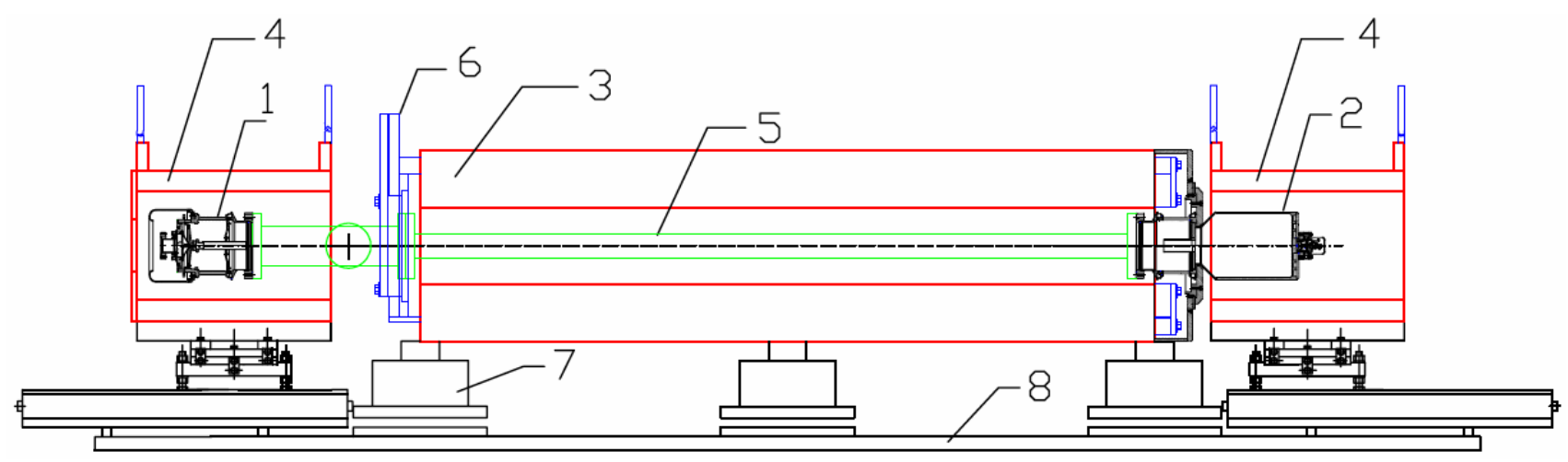

Figure 3.1: Sketch of TELTS. [24]

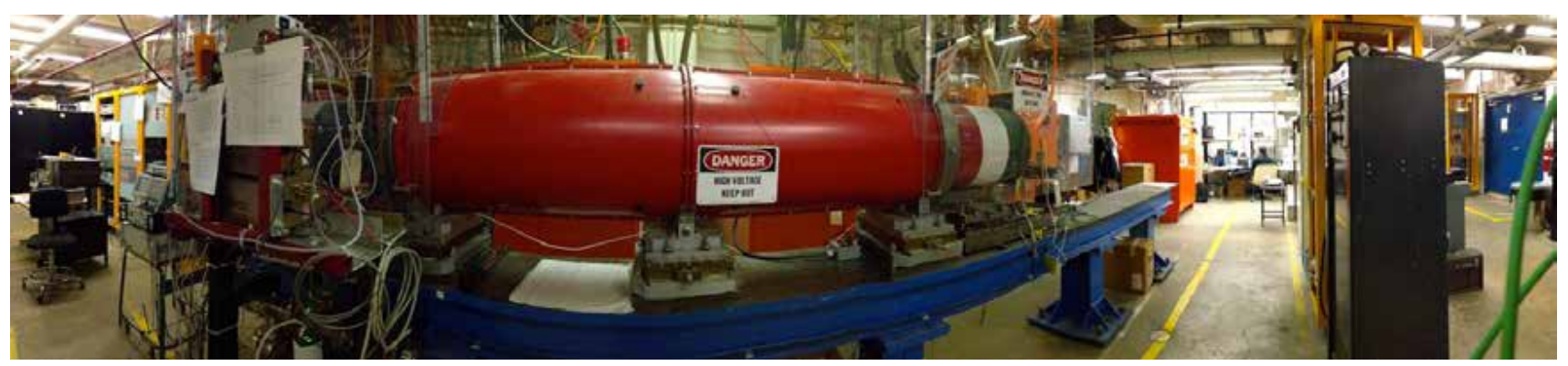

Figure 3.2: Photo of TELTS. Source: Valentina Previtali

Significant scraping could be observed at this current [11]. Similar currents are thus desired for new design considerations. For further information on the scraping studies refer to [23, 8, 11].

\subsubsection{Tevatron Electron Lens Test Stand Design Considerations}

While $H E B C$ tests with a proton beam in an accelerator are crucial, they are not intended for the characterization of the electron beam parameters. For the study of the transverse profile, the Tevatron Electron Lens Test Stand (TELTS) is used. A sketch is shown in fig. 3.1 and a panoramic photo in fig. 3.2. The TELTS is a linear test bench, where the pulsed electron gun (1) sends an electron beam downstream through a straight beam tube (5) with pickup electrodes to a water cooled collector (2). The whole beam tube is in a vacuum and is surrounded by 3 solenoids. The gun is placed in a gun solenoid (4) which is followed by a short drift space and the main solenoid (3). The collector (2) at the end of the drift tube is placed in the collector solenoid (4). Magnetic correctors (6) are used to control the beam inside the beam tube. The device is supported on a test bench (8) [24].

The distance between the gun cathode and the collector downstream is $2.86 \mathrm{~m}$. Thereof $35 \mathrm{~cm}$ are in the gun solenoid, $23 \mathrm{~cm}$ in the first drift space, $192 \mathrm{~cm}$ in the main solenoid, $15 \mathrm{~cm}$ in the second drift space and the remaining $21 \mathrm{~cm}$ in the collector solenoid. The magnetic fields in the 3 solenoids can be set independently. The gun and collector solenoid are $50 \mathrm{~cm}$ long. The inner diameter of the two short solenoids is $28 \mathrm{~cm}$ and the main solenoid is $20 \mathrm{~cm} \mathrm{[24].}$

For detailed information on the power supply, the water cooling system and the vacuum system, please refer to the Electron Lens Test Stand Wiki page ${ }^{1}$. The TELTS was used for all measurements in chap. 5 and implemented in the simulations in chap. 7.

\subsubsection{Straight Hollow Electron Beam Lens Design}

The straight hollow electron beam lens design eliminates the bends required to steer the electron beam into the accelerator beam tube in the usual electron lenses. It is thus no longer necessary for the proton

\footnotetext{
${ }^{1}$ https://cdcvs.fnal.gov/redmine/projects/elens/wiki/Test_Stand
} 
beam to pass through the hollow electron beam edge when entering the Hollow Electron Beam Lens $(H E B L)$. A sketch of such a device is shown in fig. 3.3. No such device has been built to date. In the straight hollow electron lens design, the electron gun itself is hollow, not just the cathode, and is coaxially aligned with the hadron beam of the accelerator. The electron beam is coaxial to the proton beam, focusing shortly before the main solenoid and expanding again just after. It is extracted by being radially deflected at the electron collector.

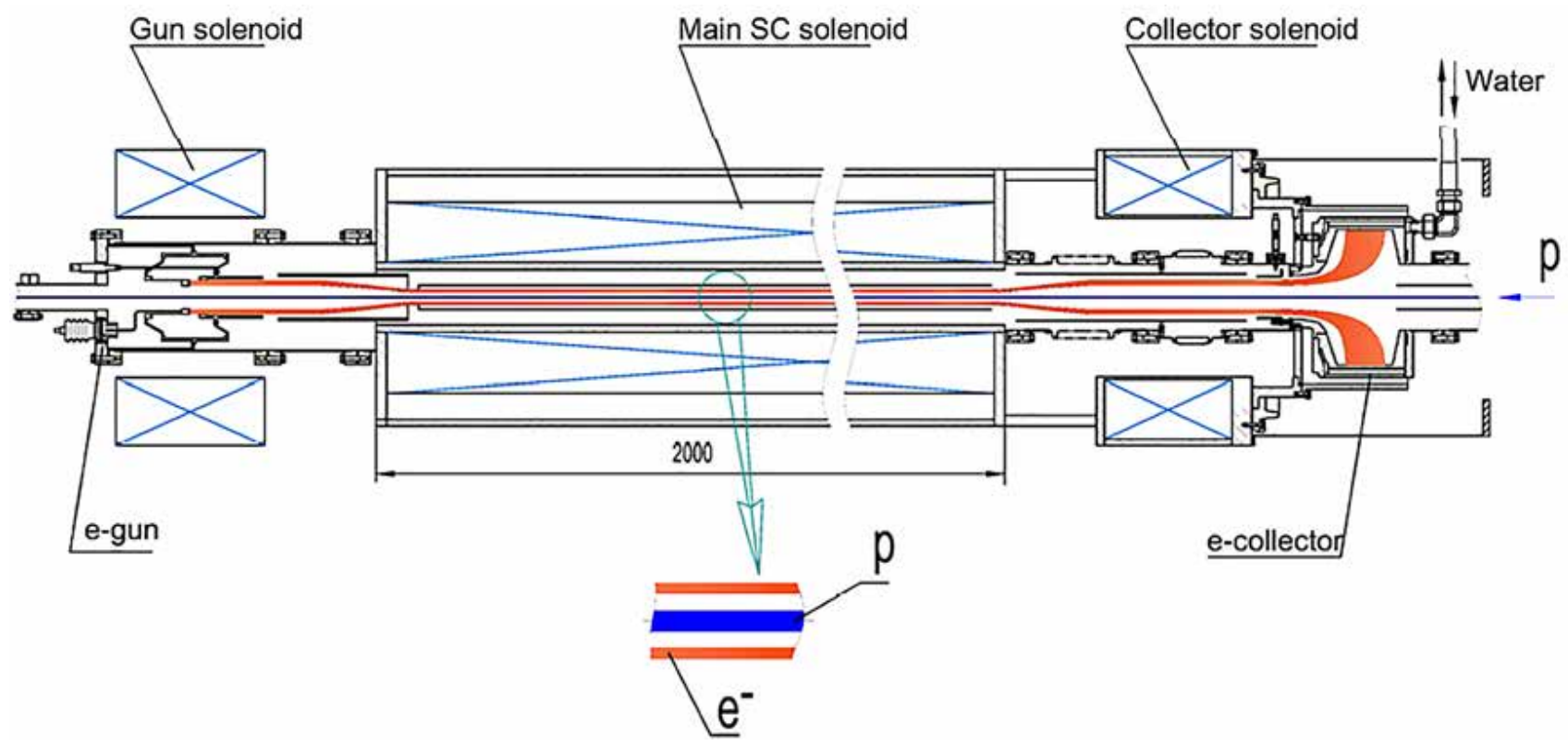

Figure 3.3: Sketch of straight HEBL setup. Source: Gennady Kuznetsov (Fermilab)

\subsection{Design of the Electron Guns}

An electron gun emits free electrons with a specific energy spectrum. The most common application is the cathode ray tube used in old television sets. Among many different electron sources, the simplest is the planar diode, consisting of two oppositely charged electrodes. The cathode emits the electrons, which are accelerated to the anode via a potential difference. The anode contains a hole or a mesh type structure to allow the electrons to pass and continue downstream of the electron gun [25, p. 7].

The most vital part of the $H E B L$ is the electron gun. Two guns were produced for the TEL2 and TELTS. First a HG06, which was used in the TEL2 setup. Second a HG1b was acquired for testing in the TELTS and subsequent installation in the $L H C$.

Both cathodes are made out of an $80 \%$ density porous tungsten matrix, impregnated with 3BaO$1 \mathrm{CaO}-1 \mathrm{AL}_{2} \mathrm{O}_{3}$. At a cathode temperature of about $2500 \mathrm{~K}\left(k_{B} T \approx 0.2 \mathrm{eV}\right)$, the work function of pure tungsten is $4.5 \mathrm{eV}$ and it produces a current density of about $0.5 \mathrm{~A} \mathrm{~cm}^{-2}$ [25, p. 9]. The work function is the minimum energy required to remove an electron from a solid to a point immediately outside the solid surface in vacuum. Using tungsten cathodes which are impregnated with barium or strontium oxides, considerably higher current densities of 10 to $20 \mathrm{~A} \mathrm{~cm}^{-2}$ can be achieved. Such cathodes are typically operated at $1400 \mathrm{~K}\left(k_{B} T \approx 0.12 \mathrm{eV}\right)$ and have a work function of $1.6 \mathrm{eV}$ [25, p. 9]. Pure barium cathodes are not used due to their low melting point of $725^{\circ} \mathrm{C}$.

\subsection{Inch Hollow Electron Gun}

HG06 was manufactured by Hi-Tech Manufacturing, LLC (Shiller Park, IL, U.S.A). The model number is 101507. The cathode is commercially available from Heat Wave Labs, Inc. (Watsonville, CA, U.S.A) [26]. It has an outer diameter of $15.24 \mathrm{~mm}(0.6 \mathrm{in})$ and a radius of curvature of $10 \mathrm{~mm}$ [26]. A $9 \mathrm{~mm}$ hole was drilled into the center of the cathode [26]. The gun is equipped with two control electrodes, 


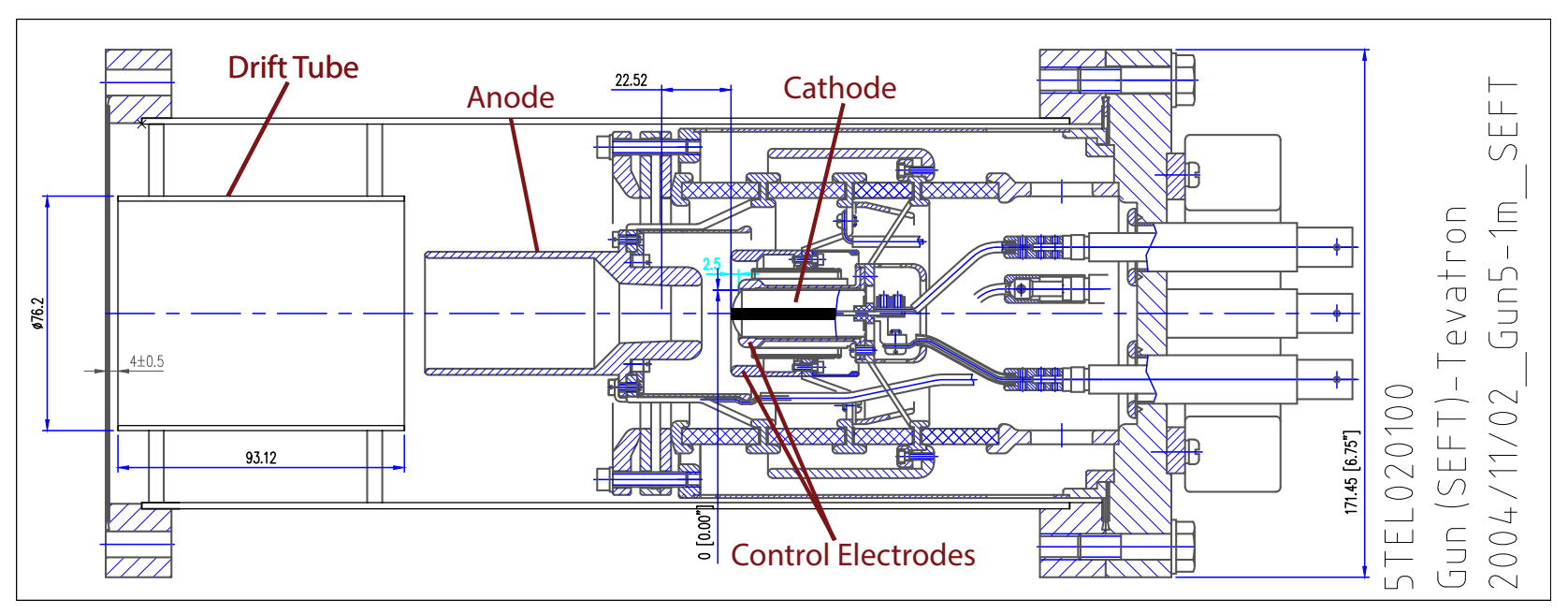

Figure 3.4: Technical Drawing of HG06. Source: Gennady Kuznetsov (Fermilab)

situated $0.28 \mathrm{~mm}$ and $9.38 \mathrm{~mm}$ from the cathode. The anode is $9.84 \mathrm{~mm}$ downstream of the cathode and has an aperture of $9 \mathrm{~mm}$ at the front. Downstream of the anode is a drift pipe with an aperture of $36.5 \mathrm{~mm}$ and a length of $93.1 \mathrm{~mm}$. Fig. 3.4 gives a technical drawing of the gun.

This gun was used for the Tevatron scraping experiments [23]. Through magnetic compression it could be scaled to a minimal inner radius of the electron beam of $0.58 \mathrm{~mm}$. The gun has a perveance of $3.20(7) \times 10^{-6}$ perv (see fig. 3.5) and an optimal operating temperature above $1100^{\circ} \mathrm{C}$ as seen in fig. 3.6. The unit perv is equivalent to the SI units $\mathrm{A} \mathrm{V}^{-\frac{3}{2}}$. Perveance is defined as the scaling factor between the beam current, $I$, and the cathode potential to the power of $1.5, V_{a}^{1.5}$. The calculated current density distribution is negligible between $0<r<4.5 \mathrm{~mm}$, rising sharply at the inner edge, and falling gradually back to 0 at the outer edge [26].

\subsubsection{Inch Hollow Electron Gun}

Given a maximum current of the HGO6 in the Tevatron of 1.2 A, a new gun was designed specifically for LHC parameters. The diameter of the cathode was increased to 1 inch in order to allow higher currents. HG1b, custom designed by Fermilab and built by Heat Wave Labs Inc., is made out of the same material as HG06. It has an outer diameter of $25.4 \mathrm{~mm}$ (1 inch), an inner diameter of $13.5 \mathrm{~mm}$ and a radius of curvature of $10.0 \mathrm{~mm}$ between the outer and inner radius. The gun is equipped with two control electrodes, situated $0.4 \mathrm{~mm}$ and $7.8 \mathrm{~mm}$ from the cathode. The

\begin{tabular}{|l|l|}
\hline$I_{\max }$ & $\geq 3.88 \mathrm{~A}$ \\
$V_{\text {sug }}$ & $3-4 \mathrm{kV}$ \\
$R_{1} / R_{2}$ & $6.75 \mathrm{~mm} / 12.7 \mathrm{~mm}=0.53$ \\
$f_{\text {comp }}$ & 3.4 \\
$B_{\text {sug }}$ & $0.43-5-0.43 \mathrm{~T}$ \\
\hline
\end{tabular}

Table 3.2: Design parameters for the e-lens installed in the Tevatron: maximum electron beam current $I_{\max }$, suggested voltage $V_{\text {sug }}$, ratio between inner and outer cathode radius $R_{1} / R_{2}$, compression required to place inner beam radius at $4 \sigma f_{\text {comp }}$ and a suggested magnetic field at the $\mathrm{LHC}$. anode is $9.8 \mathrm{~mm}$ downstream of the cathode and has an aperture of $14.3 \mathrm{~mm}$ upstream. Downstream of the anode is a drift pipe with an aperture of $36.5 \mathrm{~mm}$ and a length of $93.0 \mathrm{~mm}$. Fig. 3.7 gives a technical drawing of the gun.

The new gun can use higher magnetic fields up to $6 \mathrm{~T}$ in the beam solenoid, to improve transport of the electrons through the lens, by reducing space charge expansion and taking advantage of the $\mathbf{E}$ $\times \mathbf{B}$ drift on the electrons. One should note that design plans place the inner radius of the Hollow Electron Beam (HEB) at $\approx 4 \sigma$ of the $L H C$ proton beam. The proton beam $\sigma$ was estimated in sec. 6.3 to be $\approx 0.5 \mathrm{~mm}$ at the possible installation site of Interaction Region (IR) 4. Positioning the beam at $4 \sigma$ requires a magnetic compression of the electron beam inner radius from $6.75 \mathrm{~mm}$ to $2.0 \mathrm{~mm}$ by 


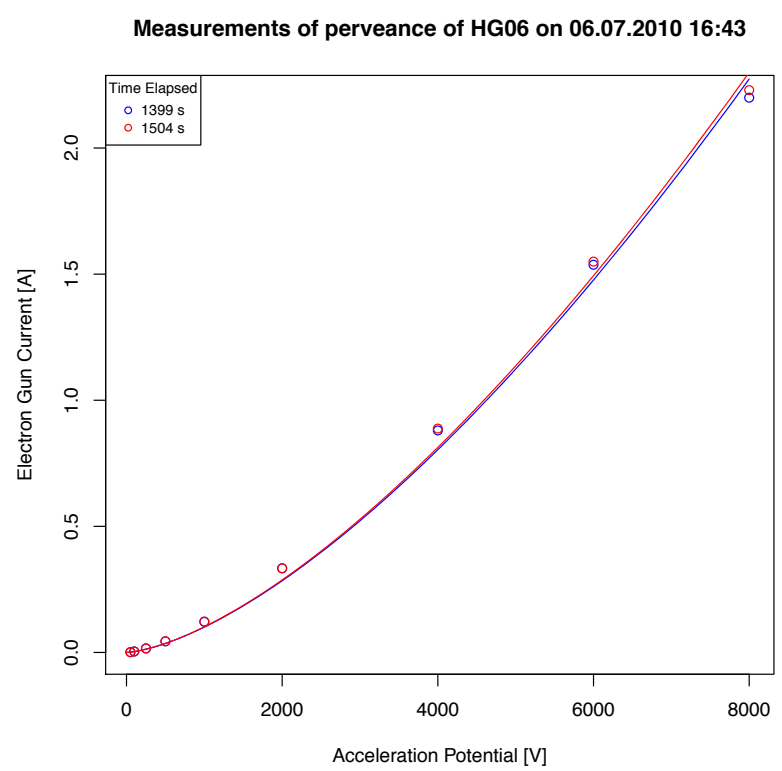

\begin{tabular}{|l|r|}
\hline Time Elapsed $[\mathrm{s}]$ & Perveance $\left[\times 10^{-6} \mathrm{~A} / \mathrm{v}^{3 / 2}\right]$ \\
1399 & $3.178 \pm 0.053$ \\
1504 & $3.214 \pm 0.051$ \\
\hline \hline Expectation & $3.196 \pm 0.074$ \\
\hline
\end{tabular}

Figure 3.5: Perveance of HG06 on 06.0\%.2010. The perveance is $(3.20 \pm 0.07) \times 10^{-6}$. Time elapsed is measured since heating of the cathode.

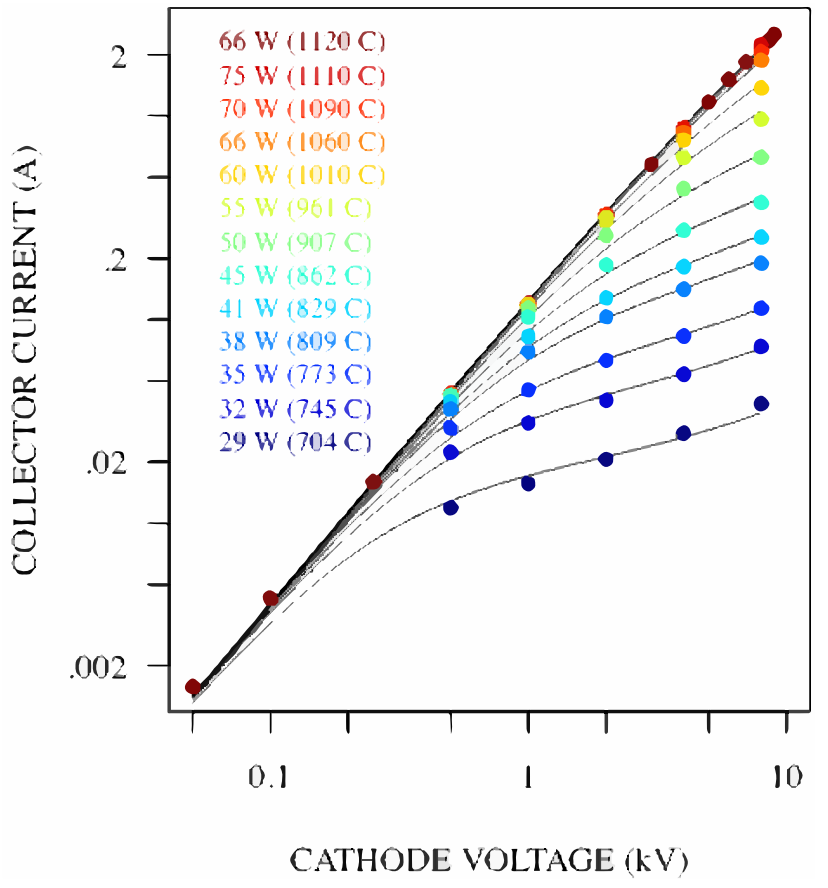

Figure 3.6: Performance of the HG06 gun with respect to cathode voltage and filament power. Source: [26]

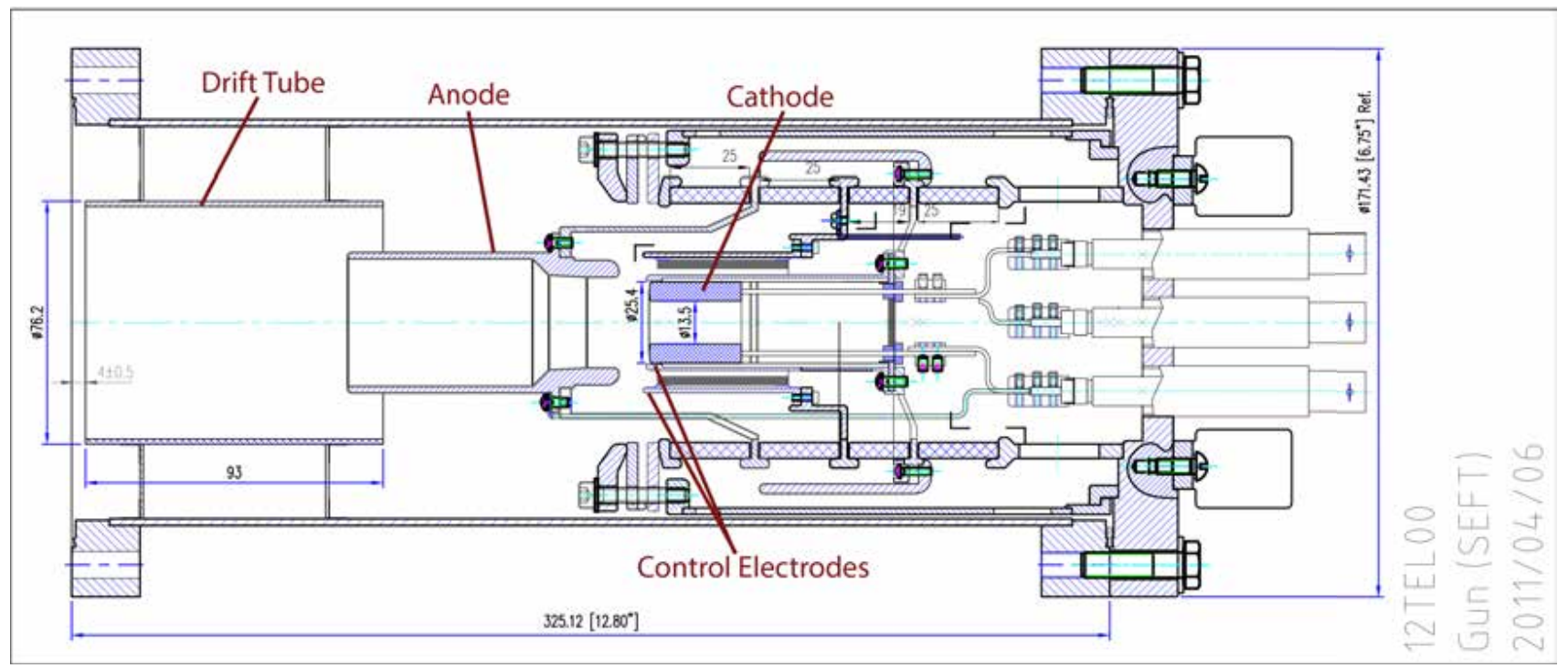

Figure 3.7: Technical Drawing of HG1b. The center of the drawing shows the cathode with the two electrodes around it and the anode and the drift pipe to its left. Source: Alexander Didenko (Fermilab contractor) 
a factor of 3.4 in the main solenoid. Given that the magnetic compression scales as $\sqrt{B_{\text {main }} / B_{\text {gun }}}$ a ratio between the magnetic field in the gun solenoid and main solenoid of $\approx 11.5$ is recommended. Given an upper limit of the magnetic field in the LHC of $6 \mathrm{~T}$, a possible magnetic configuration of the electron lens at the LHC is $0.43-5-0.43 \mathrm{~T}$. A high main solenoid magnetic field of $5 \mathrm{~T}$ is chosen for its magnetic rigidity. Given this compression, the outer beam radius would be at $\approx 3.7 \mathrm{~mm}$ or $7.4 \sigma$. Since primary collimators are placed at $6 \sigma$ [7], a large portion of the beam is shielded by the primary collimators. It might thus be possible to retract primary collimators up to $7.4 \sigma$, reducing the impedance on the proton beam. In order to obtain a similar scraping effect as was seen at the Tevatron, a cathode voltage of 3-4 kV is recommended, which provides a beam current of 1-1.5 A as can be seen in appendix tab. B.1. Furthermore, tab. 3.2 provides a list of suggested design parameters for the usage of the $H G 1 b$ at the LHC.

This gun is the focus of this thesis and was used for all subsequent measurements. HG06 was mentioned for comparison purposes and due to its significance in the Tevatron scraping experiments. 


\section{Chapter 4}

\section{Thermionic Emission}

This chapter introduces the concept of thermionic emission in Temperature Limited Emission Region (TLER) and Space Charge Limited Emission Region (SCLER). Thermionic emission governs the emission of particles from the cathode in the 1 inch Hollow Electron Gun (HG1b) and thus is directly responsible for the production of the Hollow Electron Beam (HEB). There are three main emission regimes, TLER where the beam current strongly depends on the cathode temperature, $S C L E R$ where the beam current is independent of the cathode temperature and depends on the cathode potential through Child-Langmuir law and Field Emission Regime. The latter does not apply to our guns, since it only arises at very high electric fields of $\approx 10^{10} \mathrm{~V} \mathrm{~m}^{-1}$. Another important parameter for cathode emission is secondary emission, which is induced through the passing of a primary incident particle with sufficient energy through a material. For linear beam tubes only thermionic emission is considered [27, p.39].

\subsection{Temperature Limited Emission}

With increasing temperature the probability of electrons escaping the cathode surface increases. Thermionic emission refers to the process of emitting electrons from a surface by heating that surface. The minimum energy required for an electron to escape an emitter is the sum of the Fermi energy and the work function, $E_{0}+e \varphi[27$, p. 42]. The Fermi energy is the energy difference between the highest and lowest occupied single-particle states in a quantum system of non-interacting fermions at absolute zero temperature. The work function is the energy difference between the top of the conduction band in the cathode and the vacuum level adjacent to the cathode [27]. The minimum energy required to escape an emitter is schematically shown in fig. 4.1.

Electrons obey the Fermi-Dirac distribution, meaning that each energy state may only be occupied by

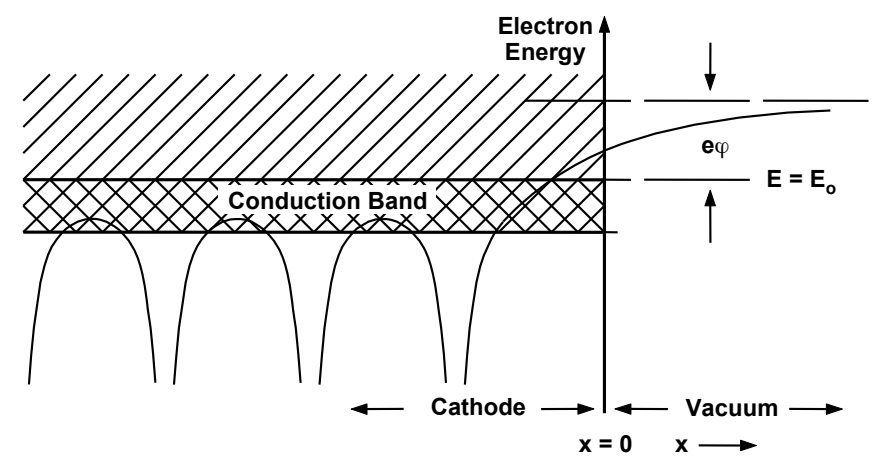

Figure 4.1: Scheme of the energy levels in a cathode. At higher energy, the disjunct energy bands merge to form the conduction band just below the Fermi Energy $E_{0}$. Particles require at least $E_{0}+e \varphi$ to escape the cathode. [27, p. 42] 
one single electron. Electrons emitted from the thermionic cathode are part of the Maxwellian tail of the Fermi-Dirac distribution [25, p. 8]. Their current density is described by the Richardson-Dushman equation:

$$
J_{\text {therm }}=A_{0} T^{2} e^{-\frac{\varphi}{k_{b} T}}
$$

where $T$ is the cathode temperature, $\varphi$ is the cathode work function and $k_{b}$ is the Boltzmann constant. $A_{0}=\frac{4 \pi e m_{e} k_{b}^{2}}{h^{3}}=1.2 \times 10^{6} \mathrm{~A} \mathrm{~m}^{-2} K^{-2}$ is a characteristic constant, where $e$ is the elementary charge, $m_{e}$ is the mass of the electron and $h$ is Planck's constant. The regime in which the emission has the above temperature dependence is called Temperature Limited Emission Regime (TLER). The beam current density is exponentially dependent on the temperature of the cathode.

Given eq. 4.1, when designing a cathode, one tries to minimize the work function in order to obtain a higher yield and possibly be able to operate at lower temperatures. The work function of our cathode and its operating temperature were discussed in sec. 3.2.

\subsection{Schottky-Effect}

Richardson-Dushman provides a simple relationship between the temperature, work function and current density, but it does not consider electric fields at the surface of the cathode. A potential difference between the anode and cathode causes charges to diffuse inside the cathode in order to return the electric field inside the conductor to equilibrium. This has the effect of lowering the effective work function by:

$$
\Delta \varphi=\left(\frac{e E_{a}}{4 \pi \varepsilon_{0}}\right) .
$$

$E_{a}$ is the applied electric field, $e$ is the electron charge and $\varepsilon_{0}$ is the electric permittivity of free space. We refer to the effective work function, since the work function is usually an intrinsic quality of the surface and material. This effect is called the Schottky Effect [27, p. 46]

Incorporating the Schottky Effect into the Richardson-Dushman equation provides the improved emission equation:

$$
J=A_{0} T^{2} e^{-\frac{e}{k_{b} T}\left[\varphi-\left(\frac{e E_{a}}{4 \pi \varepsilon_{0}}\right)^{1 / 2}\right]}=J_{0} e^{\frac{e}{k_{b} T}\left(\frac{e E_{a}}{4 \pi \varepsilon_{0}}\right)^{1 / 2}}
$$

Here $J_{0}$ is the Richardson-Dushman current density. It is used to compare the performance of different cathodes [27, p. 47].

\subsection{Space Charge Limited Emission}

Given an emitting cathode, a substantial electron cloud can build-up near the cathode. The potential outside the cathode is thus reduced by the negative charge presence of the electrons. This effect is shown in fig. 4.2. The amount of space charge at the cathode is increased when increasing the temperature in the TLER and decreased when increasing the cathode potential in the SCLER.

The electron emission rate is limited by the point where the space charge is sufficiently large to depress the potential at the cathode below zero, as is the case for (b) in fig. 4.2b. Consequently emitted electrons need to overcome a potential well, allowing only energetic electrons to be emitted from the cathode. Low energy electrons are returned to the cathode by the space charge. This causes the SCLER to be lower than the saturated emission rate, which is found exactly when the potential, and therefore the electric field, at the cathode is 0 . The saturated emission rate is equal to the Richardson-Dushman emission rate $J_{0}$, mentioned in sec. 4.2. If the potential difference between the diode increases, more electrons are emitted and in turn the potential at the cathode is decreased. If the potential difference decreases, less electrons are emitted, reducing the space charge effect and thus increasing the potential at the cathode. This effect is called the Space Charge Limited Emission Region (SCLER). During 


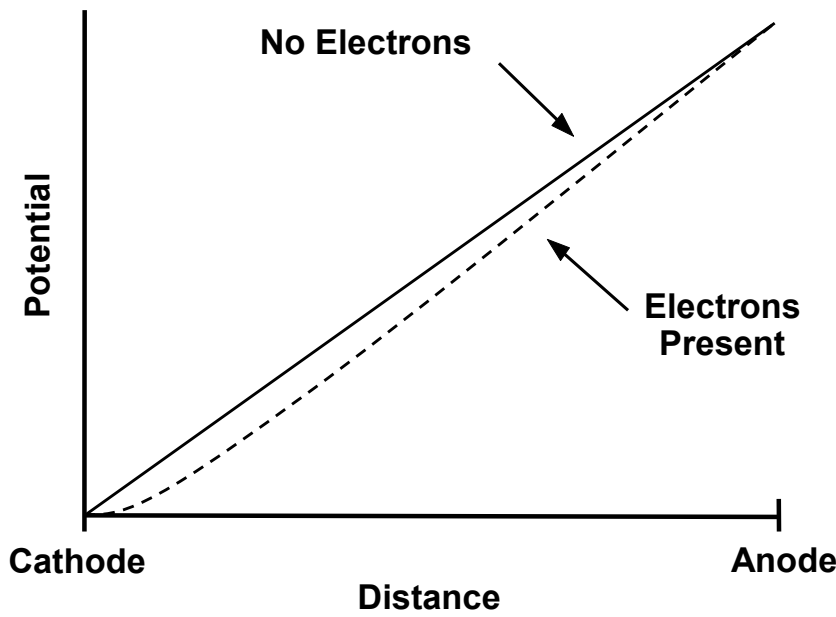

(a) If no space charge is present, the potential between a planar diode changes linearly. Because of the space charge, the gradient of the potential changes shape to that of the dashed line. Source: [27, p. 49].

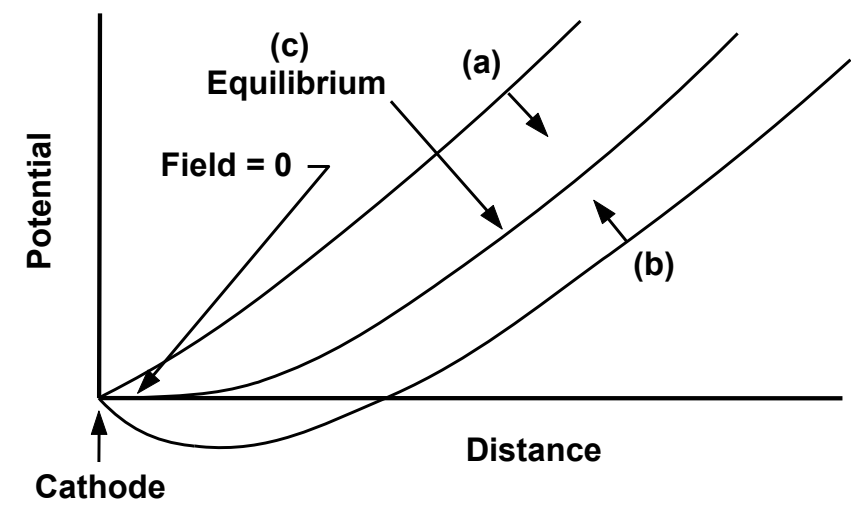

(b) As the electron emission rate is increased, the reduction of the potential continues until it reaches case (b) where the potential is negative just outside the cathode, pushing electrons back into the cathode. Source: [27, p. 501

Figure 4.2: Schemes showing the effect of space charge build-up on the potential outside a cathode.

this emission regime, cathode temperature and surface effects no longer play a role in the emission rate $[27$, p. 50]. This emission regime can be reached by increasing the cathode temperature in the TLER, thus increasing the space charge build-up at the cathode. This transition is depicted in figure $4.3 \mathrm{~b}$. The space charge limited current density is given by [25, p. 10]:

$$
J=1.67 \times 10^{-3}\left(\frac{q}{m c^{2}}\right)^{1 / 2} \frac{V_{a}^{3 / 2}}{d^{2}} \quad\left[A / m^{2}\right]
$$

where $V_{a}$ is the potential difference between anode and cathode, $d$ is the spacing between the cathode and the anode, $q$ and $m$ are the particle charge and mass and $c$ is the speed of light. All constants are given in MKS units [27, p. 51]. From eq. 4.4 the beam current of a cylindrical hollow thermionic dispenser cathode can be determined [25, p. 10].

$$
I_{\text {beam }}=1.67 \times 10^{-3} \pi\left(\frac{q}{m c^{2}}\right)^{1 / 2} \frac{V_{a}^{3 / 2}}{d^{2}}\left(r_{\text {ext }}^{2}-r_{\text {int }}^{2}\right) \quad[A]
$$

where $r_{\text {ext }}$ is the outer radius and $r_{i n t}$ the inner radius of the cathode. This law is called the ChildLangmuir law, from which a quantity, dependent on the electron gun geometry, called the perveance is defined as the ratio (distance $d$ is difficult to determine for complex diodes):

$$
P=\frac{I}{V_{a}^{3 / 2}}=1.67 \times 10^{-3} \pi\left(\frac{q}{m c^{2}}\right)^{1 / 2} \frac{r_{e x t}^{2}-r_{i n t}^{2}}{d^{2}} \quad\left[\frac{A}{V^{3 / 2}}\right]
$$

For the purpose of this thesis the unit perv is defined in SI units as $\mathrm{A} \mathrm{V}^{-\frac{3}{2}}$.

If $V_{a}$ exceeds a certain level, the space charge condition can not be upheld and the beam enters the temperature limited region, diverging from the Child-Langmuir law. This is depicted in fig. 4.3a.

Considering residual gases in the drift tubes and relativistic effects, the concept of a generalized perveance is introduced [25, p. 196].

$$
K=\frac{I}{I_{0}} \frac{2}{(\beta \gamma)^{3}}\left(1-\gamma^{2} f_{e}\right)
$$

where $I_{0}=\frac{4 \pi \varepsilon_{0} m c^{3}}{q}=17 k A$ is the characteristic current, $I$ is the beam current, $f_{e}$ is the neutralization factor and $\beta$ and $\gamma$ are the relativistic factors. Unlike the perveance, the generalized perveance is a 


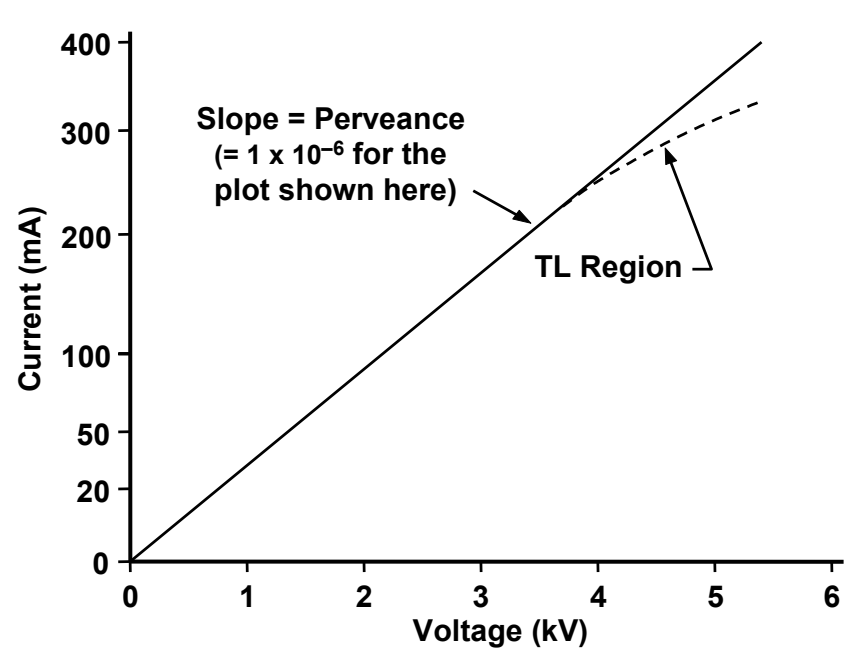

(a)In general the current density is given by the child langmuir law (see eq. 4.6 ). At high potential differences, the space charge condition is broken and the beam enters the Temperature Limited (TL) region again. Source: [27, p. 53]

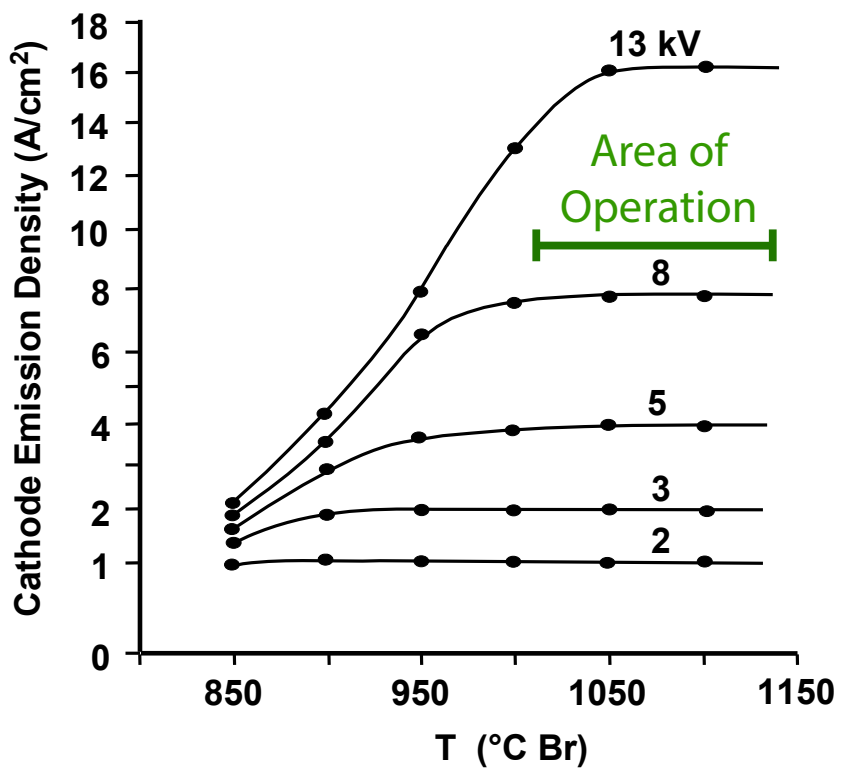

(b) With increasing temperature, the emission becomes temperature independent. The higher the cathode potential, the higher the temperature at which this transition occurs. Source: [27, p. 53]

\section{Figure 4.3: Transition from TLER to SCLER}

dimensionless quantity. Given that $\beta=\frac{v}{c}$ and $v=\left(\frac{2 q V}{m}\right)^{\frac{1}{2}}$, we can relate to $P$ by

$$
K=P \times\left[\frac{\left(1-\gamma^{2} f_{e}\right)}{4 \pi \varepsilon_{0} \gamma^{3}(2 q / m)^{1 / 2}}\right]
$$

Considering a non-relativistic situation equation 4.8 can be simplified to:

$$
\frac{K}{\left(1-f_{e}\right)}=P \times\left[\frac{m^{1 / 2}}{4 \pi \varepsilon_{0}(2 q)^{1 / 2}}\right]=P \times 1.515 \times 10^{4}
$$

Given a neutralization free beam, the correlation is $K=1.515 \times 10^{4} P[25]$.

\subsection{Field Emission}

For the sake of completion, we also introduce field emission. At electric fields much higher than that of the $S C L E R, \approx 10^{9}-10^{10} \mathrm{Vm}^{-1}$, electron emission increases rapidly, due to a low work function. Quantum mechanics thus dictates that even particles with insufficient kinetic energy to overcome the barrier by classical theory, have a non-zero probability of passing through the barrier due to the tunneling effect. Such an electron emission is called field emission. This process is schematically shown in fig. 4.4. This type of emission does not play a role in our Hollow Electron Beam Lenses (HEBLs), since we deal with a potential difference of $10^{3}-10^{4}$ over $1 \mathrm{~cm}$ and thus electric fields of $\approx 10^{5}-10^{6} \mathrm{~V} \mathrm{~m}^{-1}$. 


\subsection{Summary of Thermionic Emission}

In summary, at very low temperatures, the cathode operates in the TLER. When the temperature is high enough, a substantial space charge builds up and the emission regime transitions into $S C L E R$. This is depicted in figure 4.3. The optimal point of operation is given by the green bar and is only considered for voltages up to $8 \mathrm{kV}$. Figure 4.5 gives a summary of the various emission regions and the respective potential fields needed to achieve them. The emission from the thermionic cathode first enters the $S C L E R$, rising with voltage, until the cathode potential is strong enough in order to transport away the space charge. At this point it enters the TLER. Ultimately when the voltage is high enough, field emission takes place, since particles have a non-vanishing probability of tunneling through the work function. The optimal area of operation is again indicated

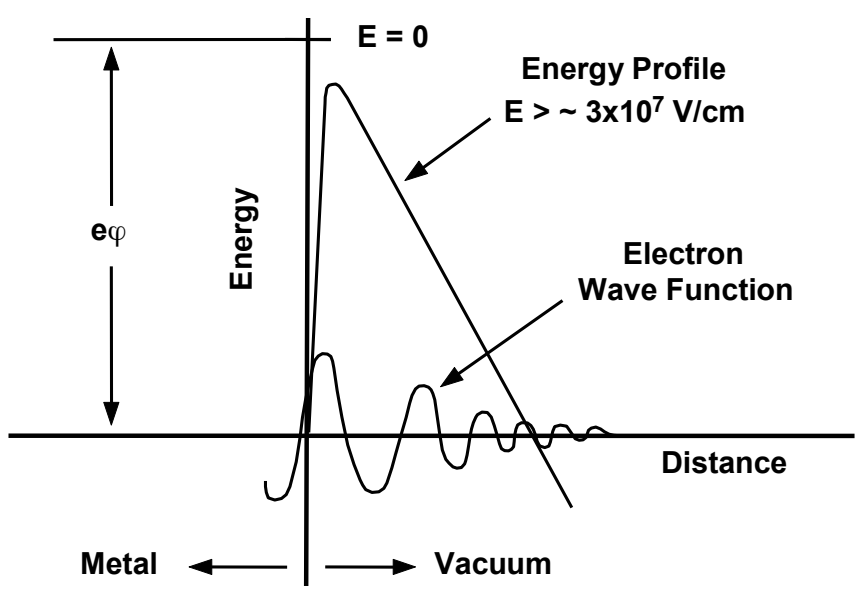

Figure 4.4: An electron with a wave function whose amplitude is lower than the potential barrier at the surface of the cathode, can tunnel through the barrier given a very strong electric field [27, p. 48]. by the green bar.

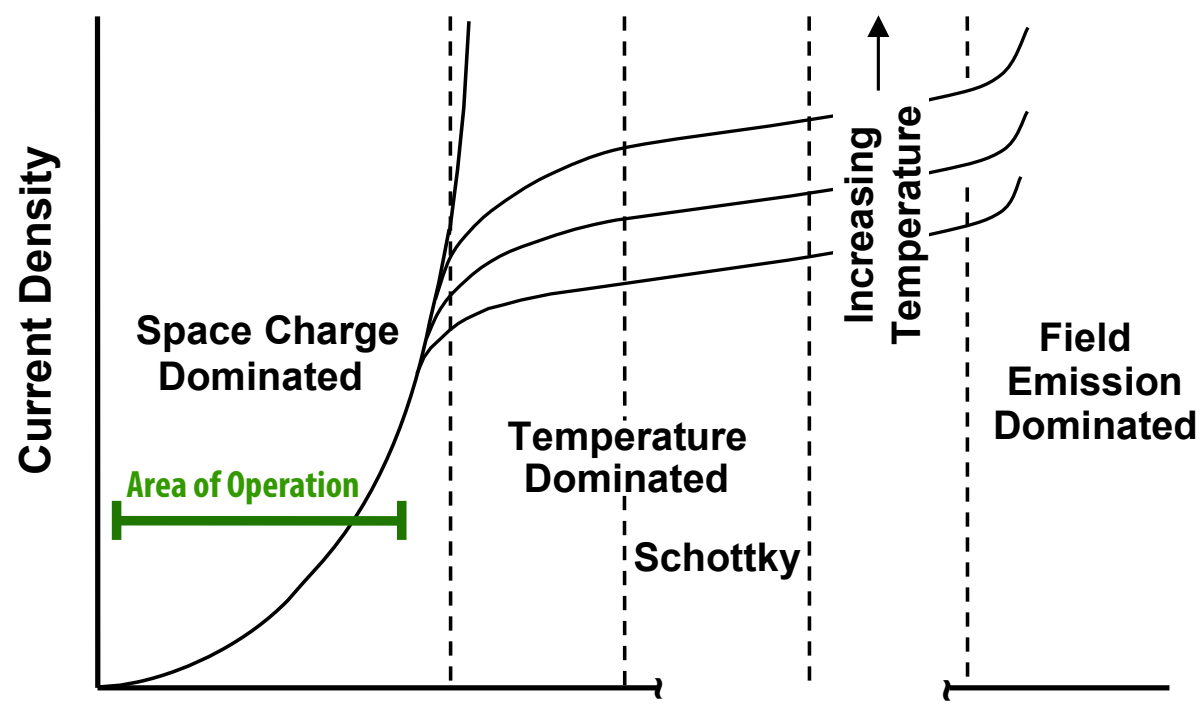

Anode Voltage

Figure 4.5: Summary of the various thermionic emission regions with increasing anode voltage. The optimal area of operation is indicated by the green bar. Source: [27, p. 55] 


\section{Chapter 5}

\section{Inch Hollow Electron Gun Characterization}

Having discussed the necessary theory, explained the terms Hollow Electron Beam Collimation (HEBC) and Hollow Electron Beam Lens (HEBL) and described the relevant electron lenses and guns, the measurements at the Tevatron Electron Lens Test Stand (TELTS) are presented. First transport measurements are discussed, these measurements describe the amount of beam that is transmitted through the main solenoid and arrives at the collectors. Then the yield measurements are discussed, a discussion of the amount of current produced by the gun. Lastly the method of transverse current distribution profile measurement is discussed and the evolution of the profiles through the beam tube with respect to the magnetic field and the cathode potential is presented.

Transport and yield measurements are done using a Tektronix TDS3034B oscilloscope ${ }^{1}$. The cathode and collector current are read using 3 induction coils, 2 dedicated to the collector current which shows a systematic error of $\approx 1 \%$. Since all induction coils are the same, this systematic error can be transferred to the cathode current readout. Additionally a statistical error on the oscillator readout of a fraction of a percent is observed $\approx 0.5 \%$. Profile measurements are made through the average current in a pinhole in the collector. The transverse profile of the beam is scanned by moving the beam over the pinhole using 6 horizontal and vertical magnetic correctors in the beam tube. Profile measurements are explained in detail in sec. 5.4. The transverse fields are calculated using a simulation code called WARP. This is discussed in detail in chap. 6.

\subsection{Operational Procedure of the TELTS}

All measurements throughout this thesis were made in the TELTS. Turning on the test stand can take 1-2 days, due to the long time it takes to heat the cathode and re-establish the vacuum. Before any parameters are changed on the TELTS, the vacuum in the beam tube is measured. The vacuum must remain below $10^{-7}$ mbar. Next the cathode is heated through a filament. The filament current is gradually increased with long pauses inbetween, so that the vacuum can recuperate. It is desirable to leave the cathode at a lower temperature than the operational temperature of $400{ }^{\circ} \mathrm{C}$ in order to evaporate all liquid constituents from the surface. The filament current is then increased to an operational temperature of $9.25 \mathrm{~A}$. Later on we will explain why this should be lowered to $8.5 \mathrm{~A}$. This process can take several days. It is important to keep an eye on the vacuum through the Fermilab Accelerator Control System (ACNET).

For future reference, we also mention the cathode temperature dependence on the filament current used to heat it. The scale of temperature to filament resistance is given in [28] as a linear local approximation:

$$
T=\beta R+T_{0}
$$

\footnotetext{
${ }^{1}$ http://www.tequipment.net/TektronixTDS3034B.asp
} 


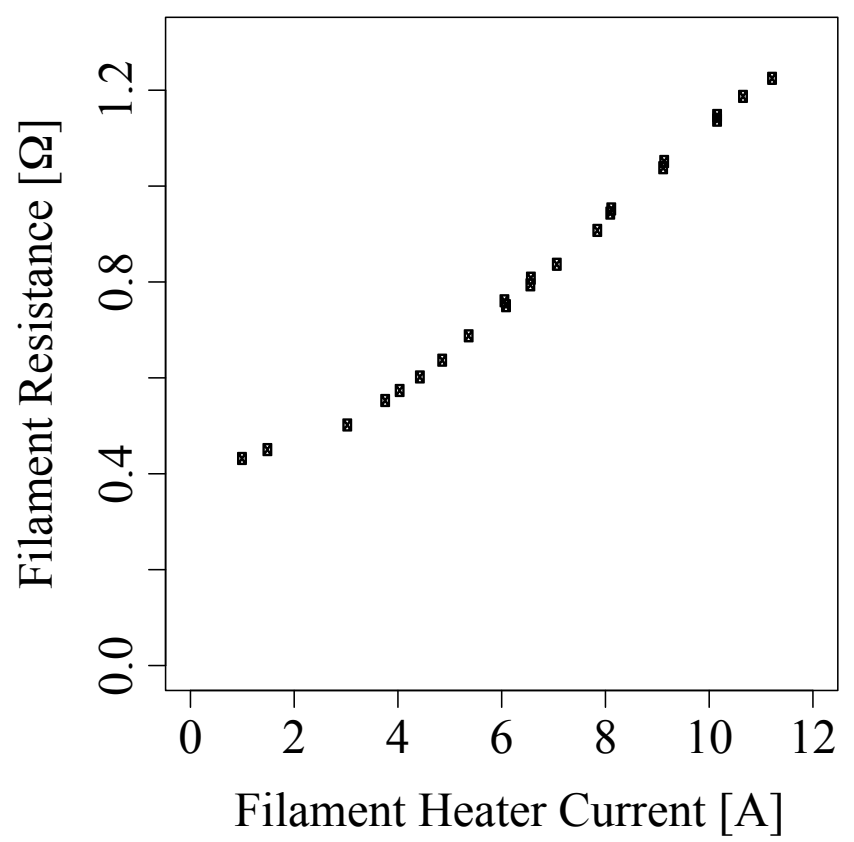

(a)R vs. I

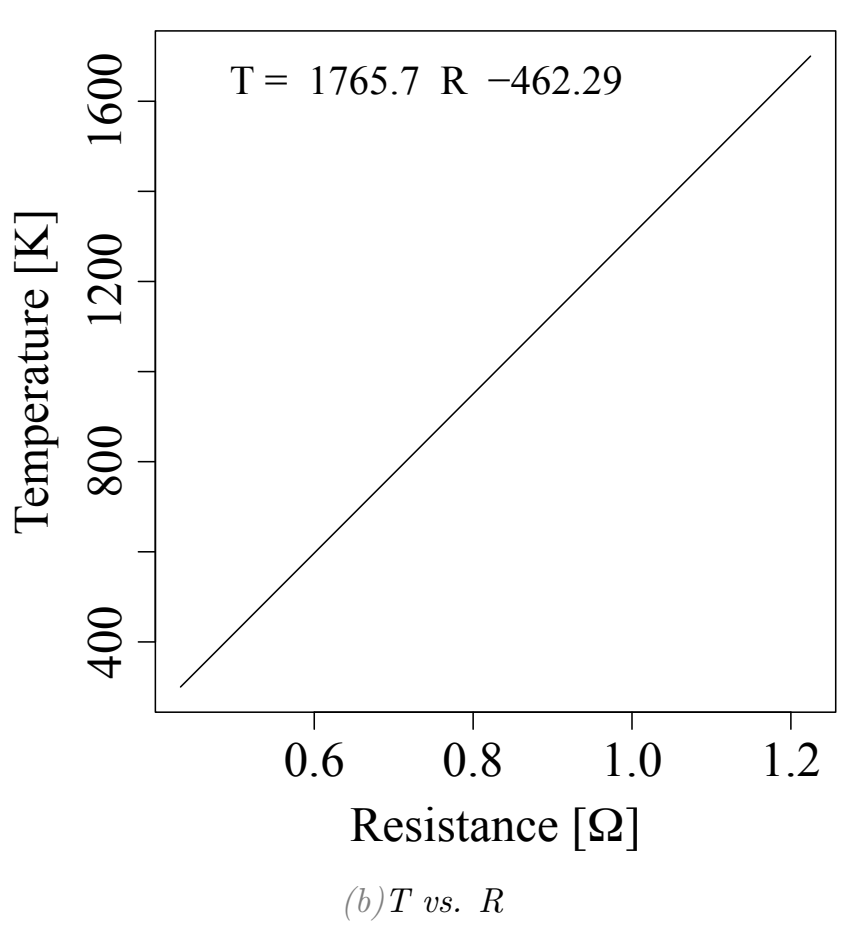

Figure 5.1: Cathode temperature in terms of filament resistance and filament current. [28]

where $\beta=1765.7 \mathrm{~K} \Omega^{-1}$ and $T_{0}=-462.29{ }^{\circ} \mathrm{C}$ and $\mathrm{R}$ is the filament resistance. The relation of filament resistance vs. filament current is given in figure 5.1a and the relation of cathode temperature to filament resistance in $5.1 \mathrm{~b}$.

Next the Tektronix TDS3034B oscilloscope is turned on. The gun, main and collector solenoids are set to the necessary magnetic fields. The main solenoid uses 2 power supplies that are linked to each other. It is thus sufficient to set the current on one of the power supplies to half that which is used to drive the main solenoid. The other power supply is set automatically. Having set the magnetic fields, the cathode voltage is set and a DEI PVX-4110 10-kV modulator is turned on in order to pulse the electron beam. The pulse width and repetition rate are set. The pulse width is usually $8 \mu \mathrm{s}$ and the repetition rate is set such that an appreciable signal is obtained.

During measurements, the pulse from the modulator is measured using 2 induction toroids at the collector, 1 induction toroid at the cathode and the pinhole in the collector of the TELTS. The data is recorded through a Programmable Logic Controller (PLC) interface for profile measurements. Beam current measurements are done using the oscilloscope.

\subsection{Transport Measurements}

Transport refers to the migration of particles or energy within a host medium, considering effects such as absorption, emission and scattering. Here the medium is a combination of beam plasma, neutralized particles and a confining magnetic field. We define transmission $T$ as the ratio of beam current at the collector to the cathode:

$$
T=\frac{I_{\text {collector }}}{I_{\text {cathode }}}
$$

This measure should ideally be $100 \%$. The new 1 inch Hollow Electron Gun (HG1b) is the largest gun that was ever used in the test stand. After a month shut-down of the e-gun in April, profiles taken on 02.05.2013 showed significant asymmetries (see fig. 5.2). Bad conditioning of the cathode surface was suspected. Following 11 additional days of conditioning at $9.25 \mathrm{~A}$ and $10.0 \mathrm{~A}$, the profiles only improved slightly. 


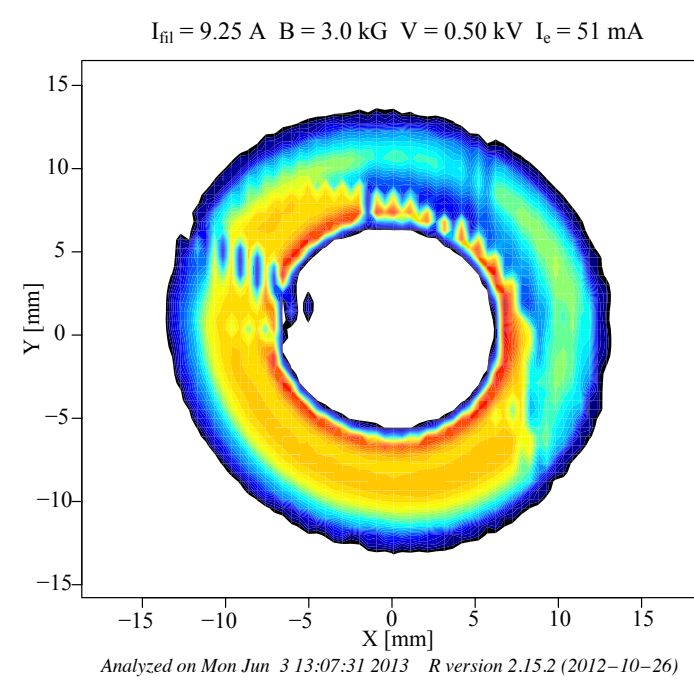

(a)02.05.2013
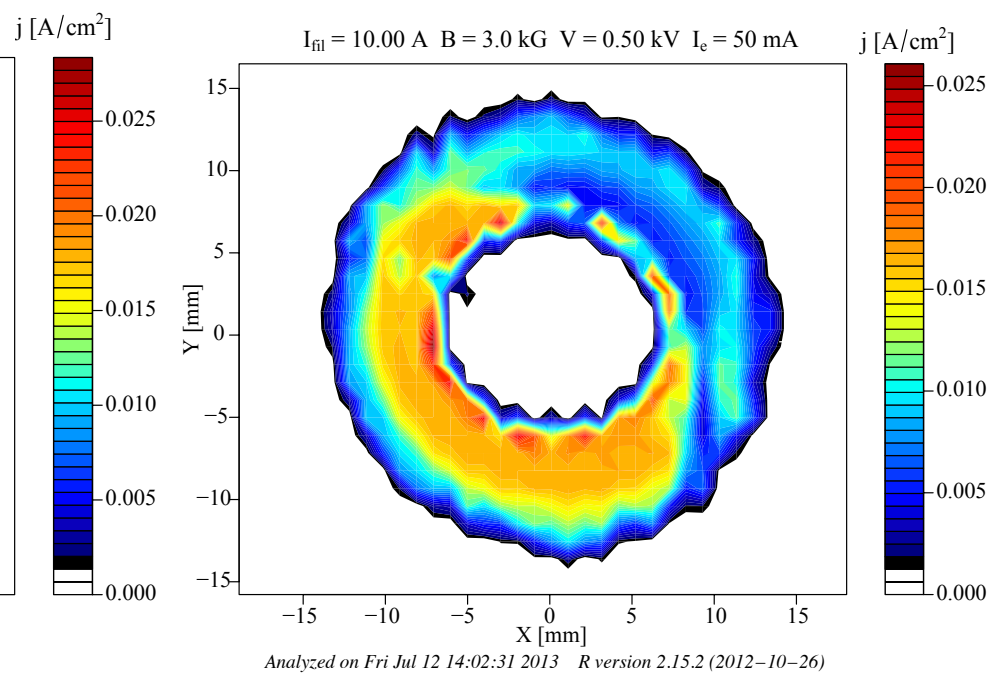

(b) 13.05 .2013

Figure 5.2: The figures show the normalized current density in $\mathrm{Acm}^{-2}$ of the measured profiles in the transverse plane. Current densities below $6.25 \times 10^{-4} \mathrm{Acm}^{-2}$ are negligible and ignored. Profile (a) shows the electron beam distribution on 02.05.2013. It is strongly asymmetric. After 11 more days of conditioning, the asymmetries have not improved as seen in (b). The production of these plots is explained in detail in section 5.4

During the follow-up a discrepancy between collector and cathode current was noted. The cathode and collector current were $68.6 \mathrm{~mA}$ and $50.6 \mathrm{~mA}$ respectively, giving a transmission of $73.8 \%$. The cathode potential was $500 \mathrm{~V}$ and the magnetic field was $0.3 \mathrm{~T}$ for this measurement. Causes for the low transmission can either be particle-particle interactions, such as neutralization, or scraping at the beam pipe. To lower the effect of scraping, the beam can be compressed in the main solenoid, and to reduce neutralization, a better vacuum could be provided.

Trying several combinations of $B_{\text {gun }}, B_{\text {main }}, B_{\text {coll }}$, it was found that transport is best with $B_{\text {gun }}=$ $B_{\text {collector }}=0.25 B_{\text {main }}$. This provides a transport of $\approx 100 \%$. Table 5.1 shows the improved solenoid settings that were used. Using the older 0.6 inch Hollow Electron Gun (HG06), such transmission issues were not observed and the gun could be used under equal magnetic fields $\left(B_{\text {gun }}=B_{\text {main }}=B_{\text {coll }}\right)$. Because the improvement in transmission for the $H G 1 b$ was achieved by compressing the beam, the most likely cause of the losses of current using equal magnetic fields is beam scraping.

\subsection{Yield Measurements}

Yield measurements are made by increasing the potential difference between the cathode and anode in regular steps and using the Tektronix oscilloscope to measure the yield at the collector. The oscilloscope averages results taken during a few seconds, reducing the statistical error significantly to a fraction of a percent. The systematic error is given by about $1 \%$.

The yield at the collector is defined by the acceleration potential $V_{a}$, the perveance $P$ and the transmission in the beam tube $T$ as:

$$
Y=P * V_{a}^{3 / 2} * T
$$




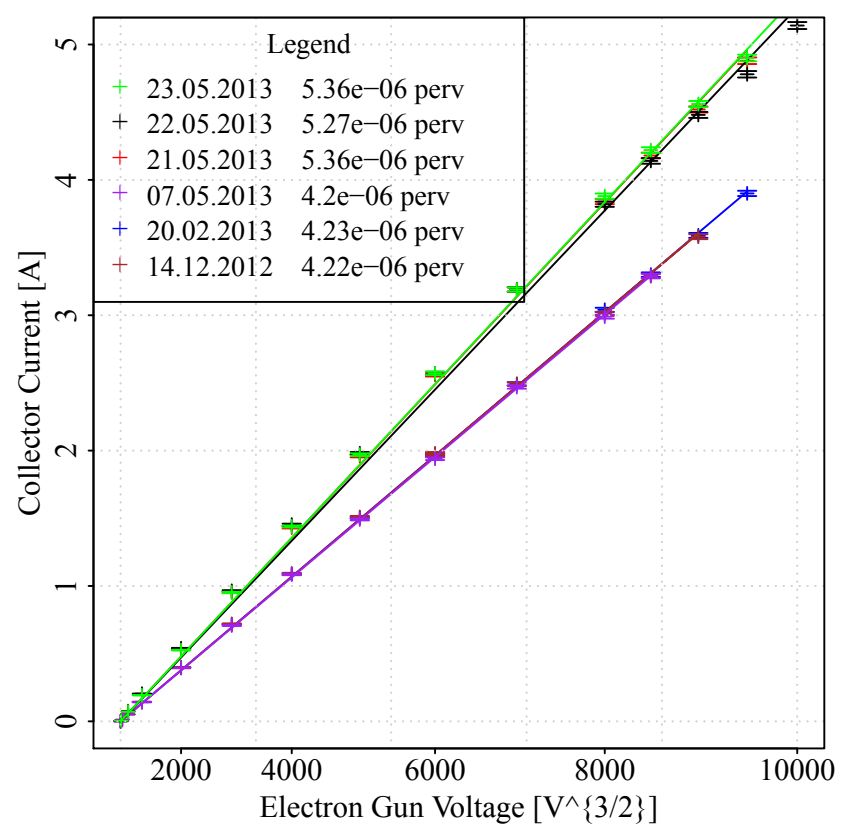

Figure 5.3: Effect of transport improvement on the yield of the test-bench. The yield follows the Child-Langmuir law and measurements are thus taken in SCLER. The statistical error is estimated at 0.5\%. Systematic errors are not considered, because they affect each measuring point equally.

\begin{tabular}{|r|r|}
\hline \hline Measurement Date & Perveance $\left[\times 10^{-6} \mathrm{~A} / \mathrm{v}^{3 / 2}\right]$ \\
\hline \hline $23.05 .201315: 02$ & $5.36 \pm 0.03$ \\
22.05 .2013 & $5.27 \pm 0.04$ \\
21.05 .2013 & $5.35 \pm 0.03$ \\
\hline \hline Expectation & $5.33 \pm 0.14$ \\
\hline \hline 07.05 .2013 & $4.20 \pm 0.01$ \\
20.02 .2013 & $4.23 \pm 0.01$ \\
14.12 .2012 & $4.22 \pm 0.01$ \\
\hline \hline Expectation & $4.22 \pm 0.03$ \\
\hline \hline
\end{tabular}

Table 5.2: Measured global perveances and their expectation values. The new measurements are $\approx 26 \%$ higher than the old perveance measurements, prior to May 2013. Error estimates are given through the statistical uncertainty and calculated by the fit.

We define the global perveance to be the fit between all data points of the peak collector current

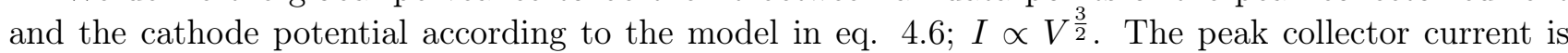
the highest current obtained from the pulsed beam shape. The pulse shape is shown in [28, p. 13]. This is an overall average which is independent of the cathode potential. In addition to the global perveance we define a quantity called the local perveance which describes the fraction of $\mathrm{I}_{\text {peak }} / \mathrm{V}^{\frac{3}{2}}$ for each measurement point.

\subsubsection{Global Perveance}

Fig. 5.3 compares the global perveance of measurements made after the transport improvements ${ }^{2}$, $5.33 \pm 0.14 \mu$ perv, and those made before the improvement, $4.22 \pm 0.03 \mu$ perv. Measurements prior to the transport improvement confirm measurements done earlier by Siqi Li [28]. Li obtained a perveance of $4.13 \pm 0.01 \mu$ perv in February 2012 and $4.15 \pm 0.02 \mu$ perv during the measurements of June 2012. The gun is relatively stable over long time periods (see fig. 5.3). All global perveance measurements after the asymmetric profiles, experienced beginning of May 2013, seem to show a slight deviation from the Child-Langmuir law. The significance of the transmission improvement is apparent through a yield increase of $\approx 26 \%$.

\subsubsection{Global Perveance from SAM Simulations}

SAM is a code developed at Budker Institute of Nuclear Physics for simulations of stationary axialsymmetric electron optical systems and electron guns. The code distributes a surface charge across electrodes and dielectrics, which are simulated using line and arc segments. A curved mesh is used to describe the space charge density which is constant within one cell. Further information on this code can be found in [29].

\footnotetext{
${ }^{2}$ Measurements continued by: G. Stancari, A. Valishev and M. Chung during the days after my departure from Fermialb.
} 


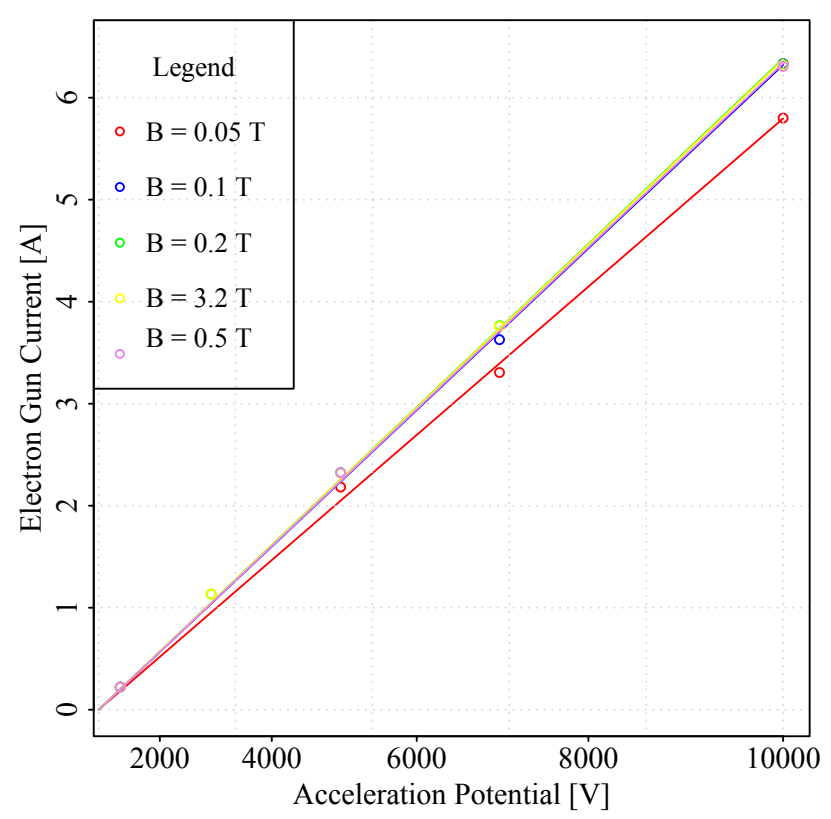

Figure 5.4: Perveance plots for the SAM simulations at 5 different solenoidal field strengths. [30]

\begin{tabular}{|r|r|}
\hline \hline Magnetic Field [T] & Perveance $\left[\times 10^{-6} \mathrm{~A} / \mathrm{v}^{3 / 2}\right]$ \\
\hline 0.05 & $5.796 \pm 0.079$ \\
0.1 & $6.322 \pm 0.057$ \\
0.2 & $6.381 \pm 0.051$ \\
0.32 & $6.371 \pm 0.051$ \\
0.5 & $6.337 \pm 0.060$ \\
\hline \hline Expectation & $6.321 \pm 0.110$ \\
\hline \hline
\end{tabular}

Table 5.3: SAM simulated perveance and expectation value of $6.3(1) \times 10^{-6} \mathrm{AV}^{-\frac{3}{2}}$.

In January 2012, using SAM, Leonid Vorobiev at Fermi National Accelerator Lab (Fermilab) simulated the electron gun current for a given acceleration potential at a magnetic field of 0.05, 0.1, 0.2, 0.32 and $0.5 \mathrm{~T}$ in the gun solenoid. From this, we calculated the global perveance of the electron gun, depicted in fig. 5.4 and table 5.3. Our measurements were within $15 \%$ of the SAM simulations and satisfactory.

The expectation value of $6.3(1) \times 10^{-6}$ perv includes all magnetic fields except that of $0.05 \mathrm{~T}$, which is insufficient to force the electrons to go on a straight path. The transverse electric fields between the cathode and anode cause the particles to undergo strong transverse oscillations, called scalloping [27, p. 138]. This results in the partial scraping of the electron beam and reduces the yield. The scalloping can be observed in fig. 5.5, where (a) is the electron beam at a solenoidal field of $0.05 \mathrm{~T}$ and (b) is at $0.32 \mathrm{~T}$. In the stronger field, the magnetic confining force is too strong to allow scalloping.

\subsubsection{Local Perveance}

Following the improvement of the transport mentioned in sec. 5.2, yield measurements were taken on 14.05.2013. Fig. 5.6a shows the electron gun peak current as a function of the cathode potential. Fitting a line to all data points (red and blue) provides us with a global perveance of $5.12 \pm 0.03 \mu$ perv. Without a discrepancy from the Child-Langmuir law, this graph should portray a straight line. This is the case up to $4000 \mathrm{~V}$. At higher voltages a gradual deviation from the Child-Langmuir law can be observed. A fit of the data up to $4000 \mathrm{~V}$ provides us with a perveance of $5.70 \pm 0.02 \mu$ perv, $\approx 90 \%$ of the SAM simulations perveance. The discrepancy between the Child-Langmuir law and the measurement is portrayed through a local perveance plot with respect to the cathode potential in fig. 5.6b.

The yield is measured at the end of the beam tube. Therefore it can be negatively affected by neutralization of the beam by residual gases in the beam tube, scraping of the beam at the beam tube edge and a departure from the SCLER to the Temperature Limited Emission Region (TLER).

When the potential is high enough, space charge effects occur further downstream, decreasing the space charge close to the gun. Consequently the SCLER cannot be sustained. Under these conditions, the emission returns to the TLER. This effect is shown in fig. 4.5 and 4.3a. Sec. 5.3.4 tells us that the voltage at which the $S C L E R$ transitions into the TLER, rises with increasing filament current. Seeing that this break down has not occurred for a filament current of $8.25 \mathrm{~A}$ in fig. 5.8c, the drop in yield 
SAM_U4.00 24-01-2012 11:56 b_05kg_135710kv__an_23_2012

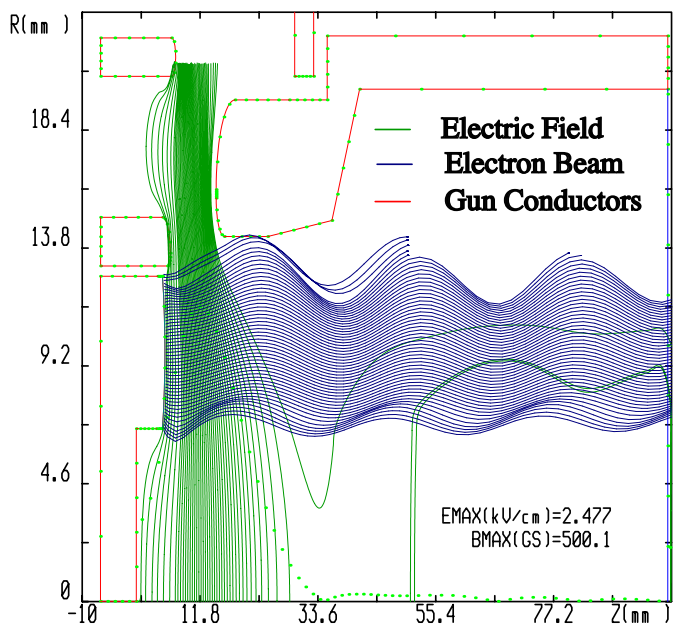

(a) SAM simulation of HG1b gun: $B=0.05 T$, $V=5 \mathrm{kV}$
SAM_U4.00 10-01-2012 12:00 jan_09_2012_5kv_ad J

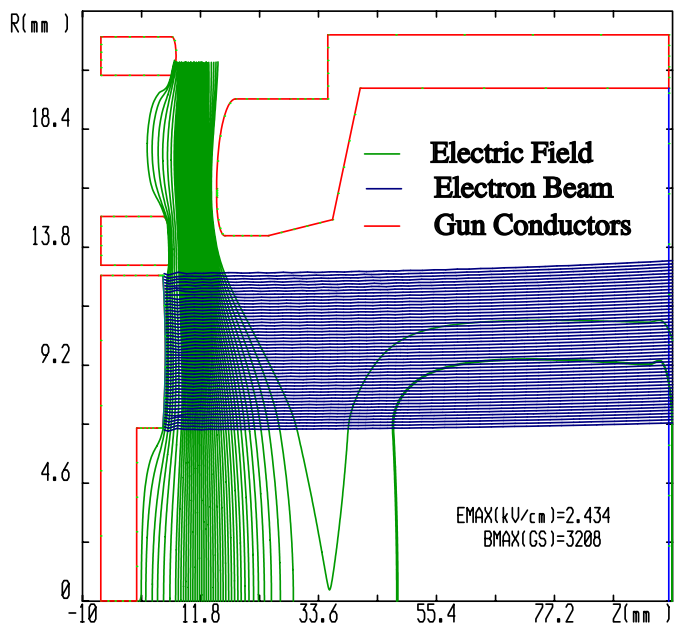

(b) SAM simulation of HG1b gun: $B=0.32 T$, $V=5 \mathrm{kV}$

Figure 5.5: SAM simulations of HG1b from January 2012 [30]. The blue lines represent the electron trajectories while the green lines represent the electric field at the cathode.
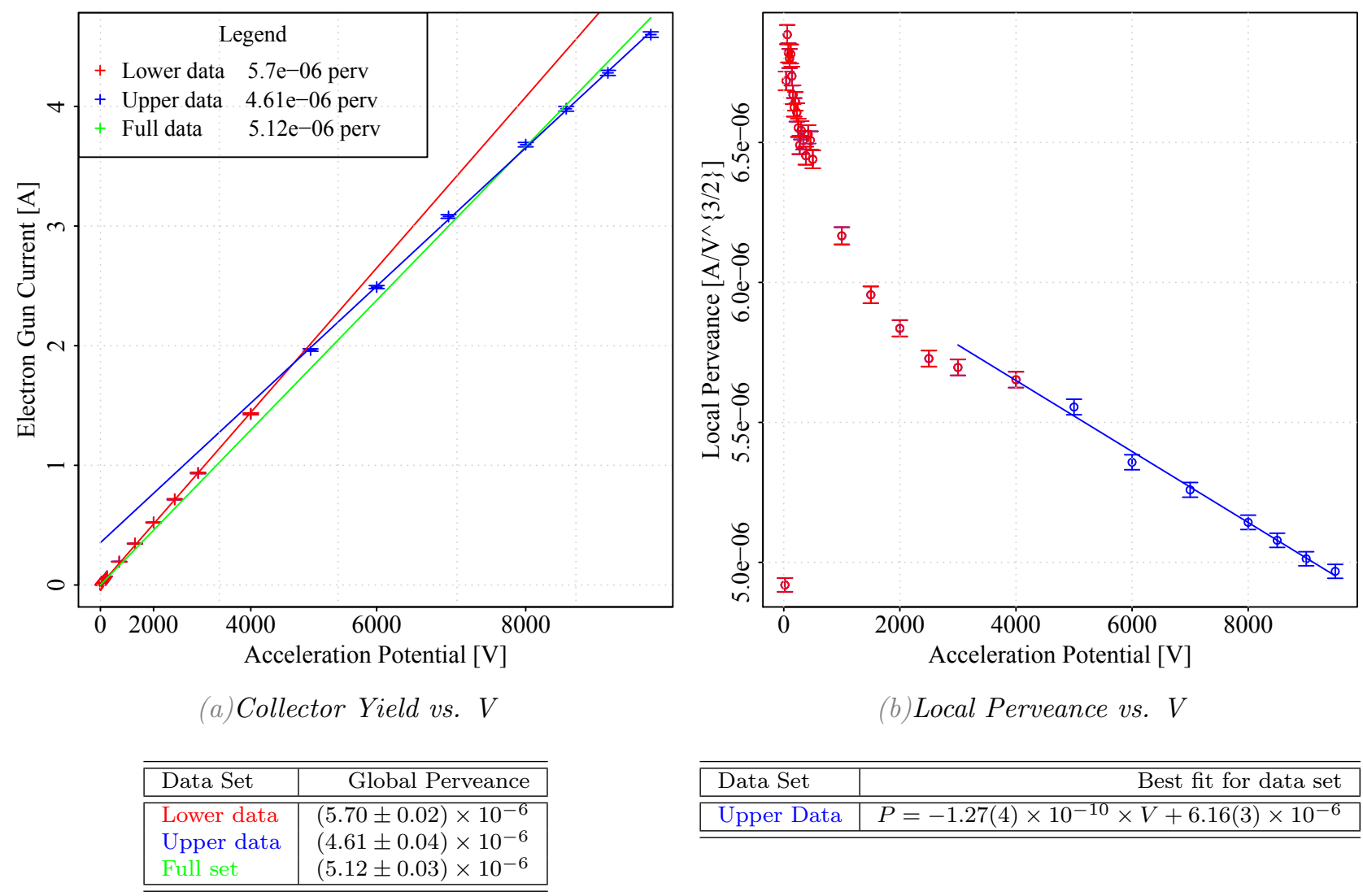

(b)Local Perveance vs. V

\begin{tabular}{|l|r|}
\hline \hline Data Set & Best fit for data set \\
\hline \hline Upper Data & $P=-1.27(4) \times 10^{-10} \times V+6.16(3) \times 10^{-6}$ \\
\hline
\end{tabular}

Figure 5.6: Global (a) and local (b) perveance measurements of the HG1b. A discrepancy was seen in fig. (a) where the perveance departed from the Child Langmuir law at $4 \mathrm{kV}$. This is most likely due to beam scraping as explained in the text. Fig. (b) shows that the change of local perveance with voltage due to this discrepancy is linear. 
seen in fig. 5.6a is probably not due to the transition into the TLER.

Neutralization refers to the absorption of electrons by background gases. There are many reasons for background gases [27, p. 173]. In the TELTS, the predominant background gas is $\mathrm{Hy}-$ drogen at $1 \times 10^{-8}$ mbar. Fig. 5.7 shows the number of ions of various gases formed per $\mathrm{cm} \times \mathrm{mbar}$ for electron-gas interactions. As can be seen, the cross section drops to values under unity when the electron energy exceeds $10^{3} \mathrm{eV}$. Assuming a pressure of $1 \times 10^{-8}$ mbar, at $5000 \mathrm{~V}$ only $5 \times 10^{-8}$ ions are formed per $\mathrm{cm}$. We can thus safely assume that neutralization only plays a role for low energy electrons, below $4000 \mathrm{~V}$. This was also confirmed in Siqi's paper [28].

The sudden change of slope between $4 \mathrm{kV}$ and $5 \mathrm{kV}$ in fig. $5.6 \mathrm{a}$ is thus likely explained by the scraping of the beam at the beam tube with a radius of $3 \mathrm{~cm}$. The effect thereof seems to be a linear change of perveance with voltage after $4 \mathrm{kV}$

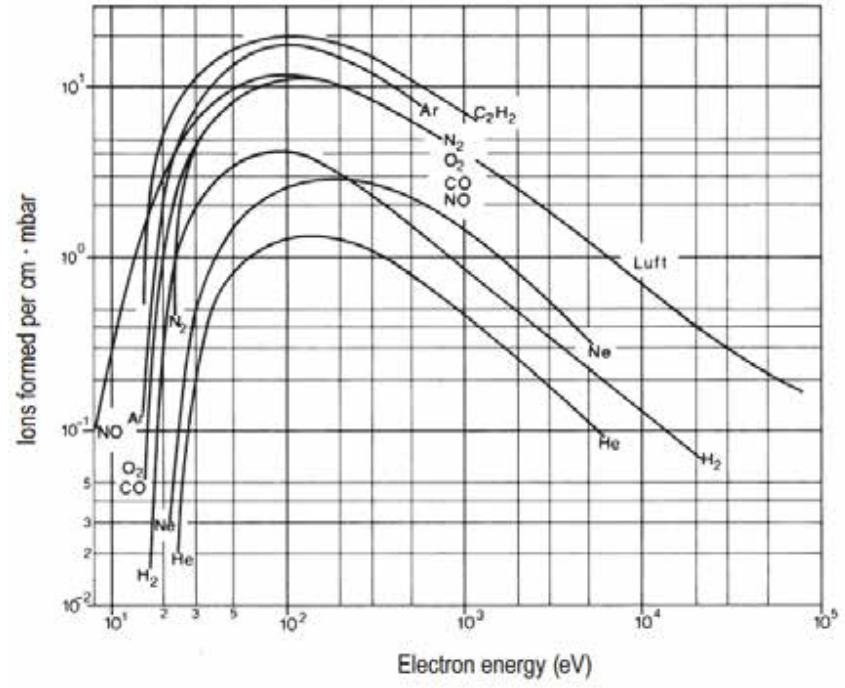

Figure 5.7: Number of ions formed per cm per mbar for various gases. Source: [31, p. 104] with a slope of $-1.27(4) \times 10^{-10}$ perv $\mathrm{V}^{-1}$, as seen in fig. 5.6b. The linear change could be explained by the beam reaching a transverse beam size at $V_{a}=4 \mathrm{kV}$ such that the beam starts scraping on the beam tube edge. As the acceleration potential $V_{a}$ is increased, more current is produced and the scraping increases, further lowering the perveance.

Assuming that the decrease in yield is mostly due to scraping, the fit for the whole data set (green) in fig. 5.6a is the best assumption for the yield at the collector. This gives a perveance of $(5.12 \pm 0.03) \mu$ perv The fit for the set of lower data best describes the perveance of the gun itself, which excludes losses downstream of the gun.

\subsubsection{Dependence of Perveance on Filament Current}

This section discusses the filament current dependence of the perveance. A scaling law between filament current and temperature of the cathode is given in eq. 5.1.

Additional measurements were made at a magnet setting of 1-4-1 kG $\left(B_{\text {gun }}-B_{\text {main }}-B_{\text {coll }}\right)$ for linearly changing filament currents from $6.25 \mathrm{~A}$ to $9.75 \mathrm{~A}$ in steps of $0.5 \mathrm{~A}$. Using this data, we expect to be able to validate our current measuring point of $9.25 \mathrm{~A}$ and possibly deduce a lower filament operating current, without a significant loss in yield due to the temperature-limited regime. The cathode yield was acquired at a repetition rate of $4 \mathrm{~Hz}$. Fig. 5.8 shows the measurement results.

Fig. 5.8a shows the transition from SCLER to TLER and the voltage at which this transition occurs. In the TLER, the emission current is independent from the cathode potential, when the emission has entered the TLER completely. The transition does not take place below $10 \mathrm{kV}$ for a filament current between $8.25 \mathrm{~A}$ and $9.75 \mathrm{~A}$. Interestingly, the emission current seems to drop again at higher cathode potentials as one surpasses $8.75 \mathrm{~A}$ of filament current. This effect is analyzed more closely in fig. 5.9a.

Fig. 5.8b shows the electron gun current as a function of the filament current. Considering measurements on the full potential voltage scope $(0.1-10 \mathrm{kV})$, the maximum "knee current" is at $8.25 \mathrm{~A}$. The "knee current" is given by the filament current at which the transition into the SCLER takes place. If we consider a safety filament current increment of $0.5 \mathrm{~A}$, an operating filament current of at least $8.75 \mathrm{~A}$ is recommended. If one wishes to only measure profiles with a cathode potential of up to $7 \mathrm{kV}$, this recommended filament operating current can be lowered to $8.25 \mathrm{~A}$ and for up to $4 \mathrm{kV}$ to $7.75 \mathrm{~A}$. The filament current increment is used, because the yield tends to fall with time as described in [27, p. 73]. By taking a safety filament current increment, we guarantee that the current density produced by the cathode does not fall and measured profiles are consistent. 

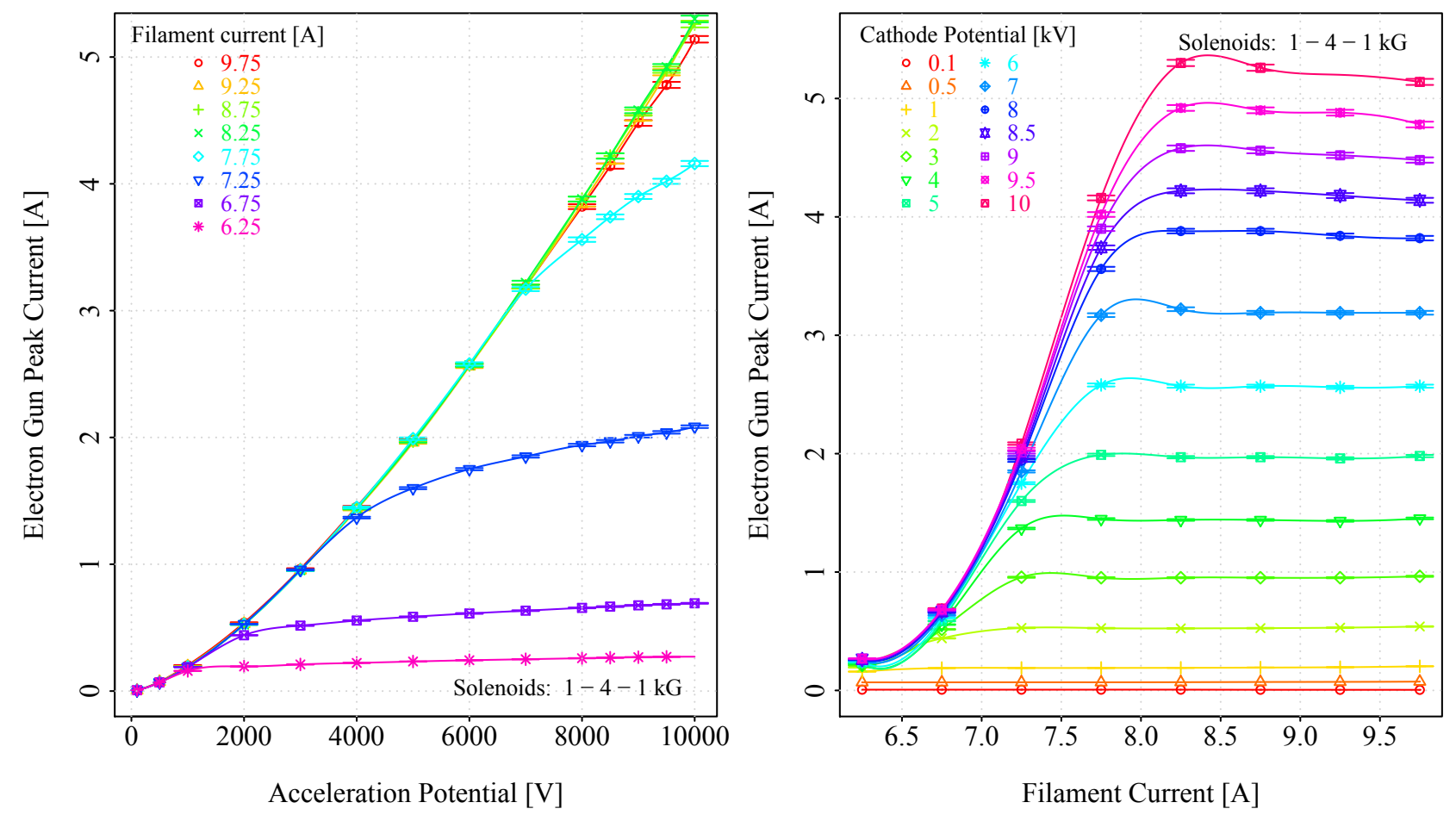

(a)Peak current vs. cathode potential for various filament currents.

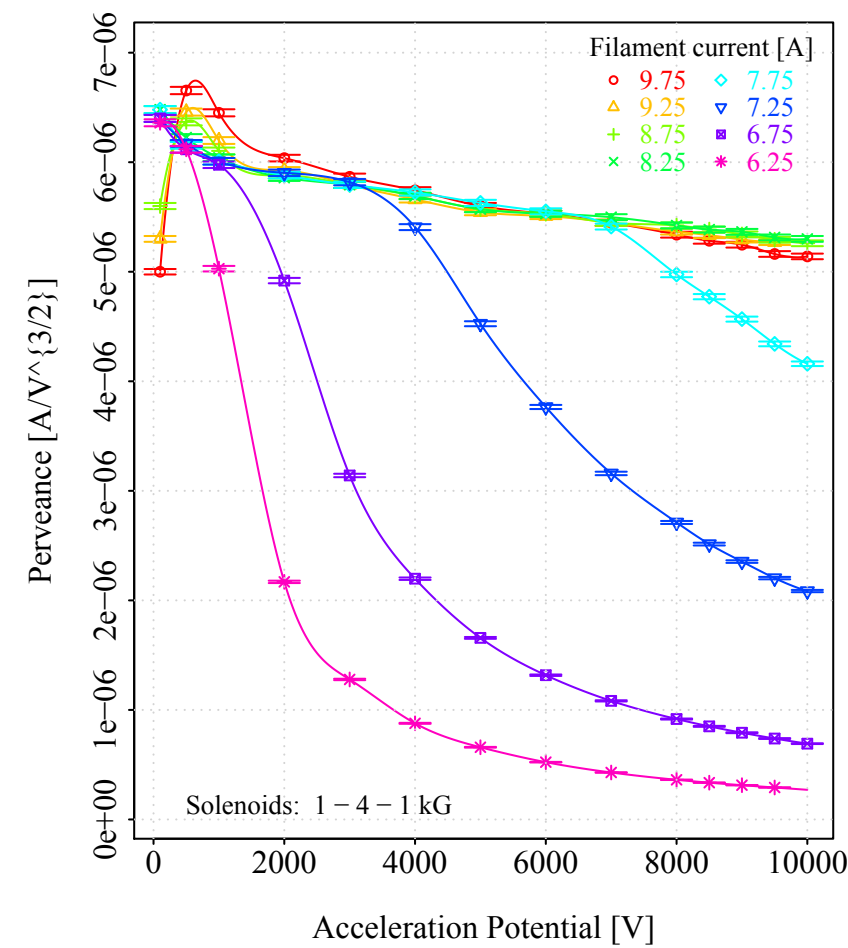

(b)Peak current vs. filament current for various cathode potentials.

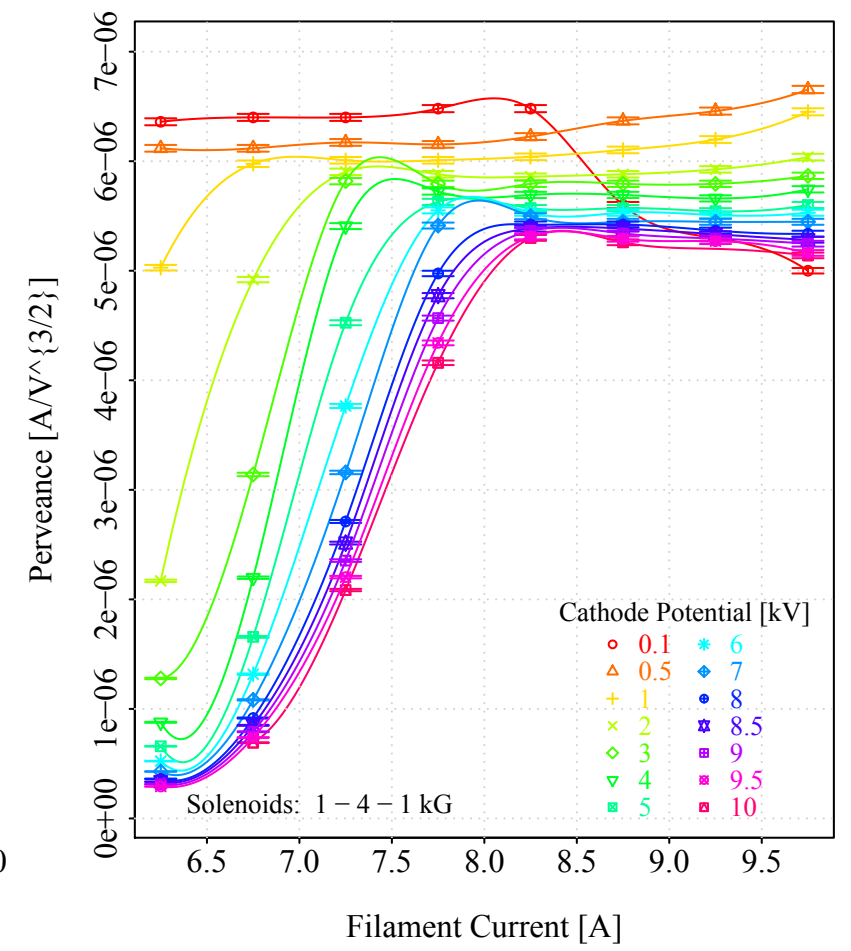

(c)Perveance vs. cathode potential for various filament (d)Perveance vs. filament current for various cathode currents. potentials.

Figure 5.8: Electron gun peak current (top graphs) and perveance (bottom graphs) as a function of acceleration potential and filament current. All measurements were taken at 1-4-1 kG at a repetition rate of $4 \mathrm{~Hz}$. 


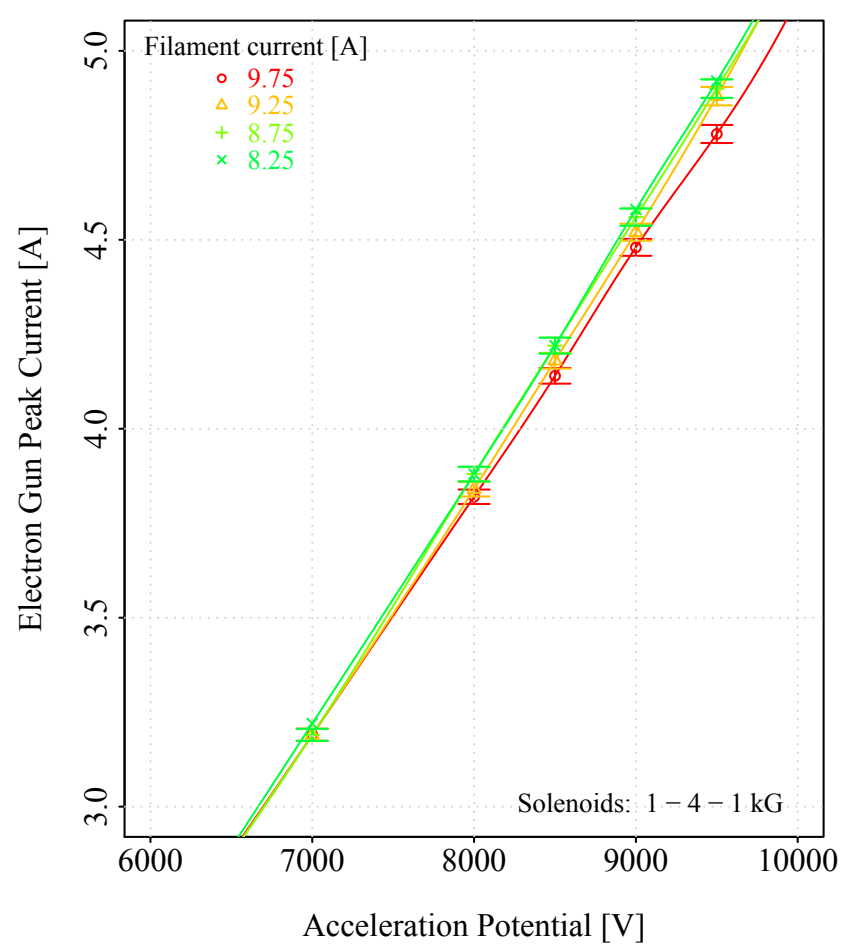

(a)Close-up of peak current vs. cathode potential for the highest filament currents.

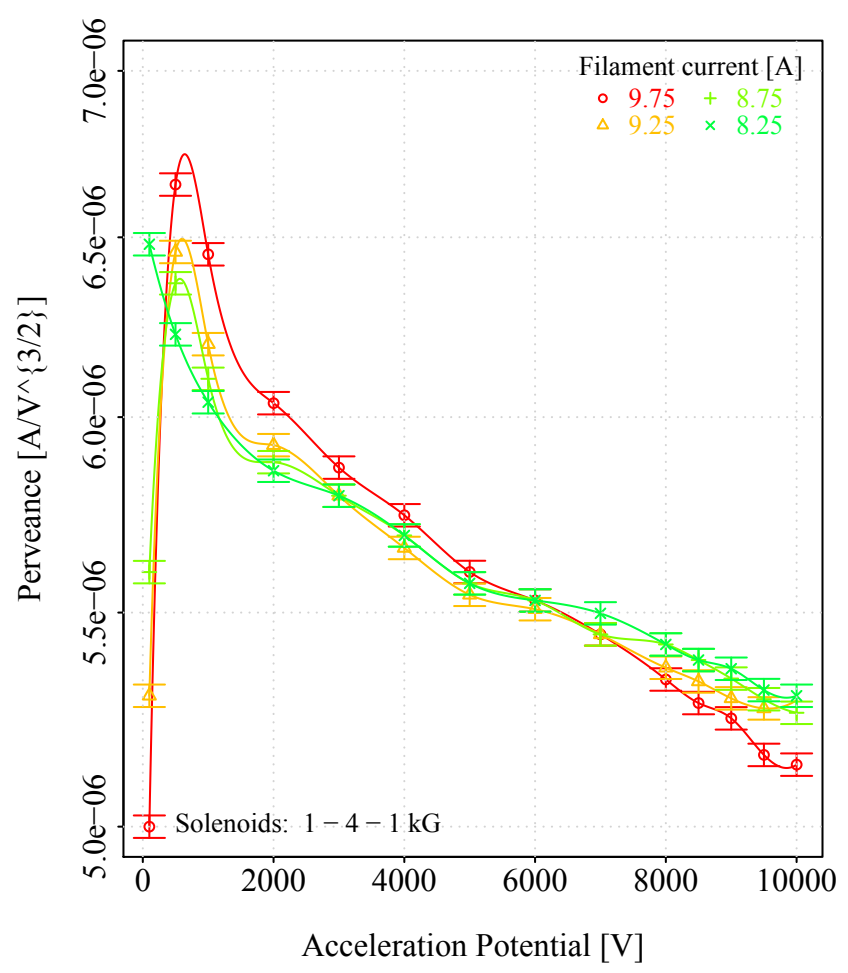

(b)Close-up of perveance vs. cathode potential for various filament currents.

Figure 5.9: Close up of the cathode-potential dependence of the electron gun peak current and the perveance. The left plot shows that the yield is actually lower for the higher temperature cathode with a filament current of 9.75 A. The right plot, allows one to deduce what temperature to use at given cathode voltages. Below $6 \mathrm{kV}$, the yield is greatest for the highest filament current of $9.25 \mathrm{~A}$. Above $6 \mathrm{kV}$, the yield is highest for a filament current of 8 to $9 \mathrm{~A}$.

Fig. 5.8c, similar to fig. 5.8a, shows the transition into the TLER and the drop in perveance. This plot confirms that the local perveance drops slightly at high cathode potentials when exceeding $8.75 \mathrm{~A}$ filament current and that the cathode should be operated above 8 A. Fig. 5.8d shows the transition of the perveance from TLER to SCLER.

Fig. 5.9 shows that above a given filament current, the yield is lower than for smaller filament currents. The perveance at $9.75 \mathrm{~A}$ falls below that of $8.75 \mathrm{~A}$ and $8.25 \mathrm{~A}$ at $6 \mathrm{kV}$ and the perveance at $9.25 \mathrm{~A}$ falls below that of $8.75 \mathrm{~A}$ and $8.25 \mathrm{~A}$ at $3 \mathrm{kV}$ as seen in fig. $5.9 \mathrm{~b}$. Running the cathode at a too high temperature will deplete the $\mathrm{BaO}$ in the porous tungsten faster and decrease the lifetime of the cathode [27, p. 63].

The above analysis proposes that one operates the cathode not at $9.25 \mathrm{~A}$, as done up to now, but at a lower filament current of $\approx 8.5 \mathrm{~A}$.

\subsubsection{Generalized Perveance}

The generalized perveance provides a dimensionless unit used to compare different cathodes (sec. 4.3). In order to calculate this quantity, we first need to assert that we can operate in non-relativistic assumptions. Given the simple relation $\gamma=\frac{E}{E_{0}}$ one can derive a relation for the velocity of a particle having passed the anode.

$$
\sqrt{\frac{1}{1-\beta^{2}}}=\gamma=\frac{E_{0}+q V_{a}}{E_{0}} \Rightarrow \beta=\sqrt{1-\left(\frac{E_{0}}{E_{0}+q V_{a}}\right)^{2}}
$$


$E_{0}$ is the rest energy of the electron, $\gamma$ and $\beta$ are relativistic parameters, $q$ is the electron charge, $V_{a}$ is the acceleration potential and $E$ is the total energy of the electron, which is given by the acceleration potential. The electron velocity is given by $\beta c$, where $c$ is the speed of light. Using conservative values for $V_{a}$ of $8000 \mathrm{~V}$, which is the maximum we have measured, we obtain a $\beta$ of 0.17 and can thus proceed by calculating the general perveance in a non-relativistic manner.

In order to determine the general perveance (see eq. 4.9), we would plot the right hand side of that equation vs. the voltage and see that the right hand side equals the general perveance when the neutralization is 0 . Sigi Li's paper [28, p. 15] states that the neutralization approaches 0 at very high acceleration potentials. An upper limit for the generalized perveance is determined by utilizing the local perveances at $10 \mathrm{kV}$ from fig. $5.9 \mathrm{~b}$, where neutralization is clearly 0 . Averaging these values gives a local perveance of $5.14(5) \times 10^{-6}$ perv. Using eq. 4.9 the generalized perveance is $7.93(7) \times 10^{-2}$. This quantity will be important when comparing the measurements to the numerical simulations in chap. 7 .

\subsection{Profile Measurements}

Transverse profiles are measured in order to analyze the transverse beam distribution and any azimuthal asymmetries in the transverse profile. They are subsequently used to determine the transverse electric fields of the measured profiles and an upper limit for the emittance growth due to the HEBL.

\subsubsection{Transverse Profile Measurement}

Transverse profile measurements are acquired using 6 horizontal and 6 vertical beam correctors that deflect the beam in the transverse plane. A $0.2 \mathrm{~mm}$ pinhole at the center of the collector measures the current density as the beam is moved along a grid, defined in the acquisition script (ACL). The readout is then saved as a text file and processed by a R Script. This chapter discusses the profile measurement method and the profile evolution as it travels through the beam tube. Additionally a scaling law is deduced and discussed.

\section{ACL Script}

The full Accelerator Command Language (ACL) script is provided in appendix C.1.1.

An $A C L$ script [32] controls the TELTS through the ACNET for profile scans. After initializing the run, the script defines the list of horizontal and vertical correctors that are used and the variables that are to be read (Code Line: 18) For future reference the most important variables are listed in table 5.4. It then defines the extension of the grid over which the scan is to be performed. This grid size is given in units of ampere.

\begin{tabular}{|r|r|}
\hline \hline Variable Name & Variable Descriptor \\
\hline Z:BC1AD3 & probe current \\
Z:BC1AD5 & tube current \\
Z:BC2AD5 & diode pump vacuum \\
Z:HCORxx & horizontal corrector positions \\
Z:VCORxx & vertical corrector positions \\
\hline
\end{tabular}

Table 5.4: List of variables recorded by ACNET and saved during the transverse profile measurements. It then defines a quantity called step which gives the step size made in each direction. The total size in horizontal or vertical direction of the grid has to be an integer multiple of the step size. The variable $d t$ defines how much time is spent per measurement point. For post transmission upgrade measurements a $31 \times 31$ pixel grid with 2 s interval per pixel were used. Pre-transmission upgrade measurements used a $61 \times 61$ pixel grid with a $1 \mathrm{~s}$ interval per pixel. The change was made purely for the purpose of reducing measurement time. Table 5.5a shows the optimal grid sizes in A for the various magnetic field configurations. Additionally tab. 5.5b gives the correction parameters for the grid not being centered around $(0,0) \mathrm{A}$.

The script first runs a few safety calculations (Code Line: 55) to see that all entries are correct and physical. After this it proceeds by calculating the expected runtime. For our measurements this was usually of the order of one hour. Lastly the script defines a quantity called stepsign (Code Lines: 


\begin{tabular}{|l|l|l|}
\hline \hline $\begin{array}{l}\text { Solenoidal config- } \\
\text { uration }[\mathrm{T}]\end{array}$ & $\begin{array}{l}\text { Horizontal set- } \\
\text { tings }[\mathrm{A}]\end{array}$ & $\begin{array}{l}\text { Vertical settings } \\
{[\mathrm{A}]}\end{array}$ \\
\hline \hline $1.0-1.0-1.0$ & {$[-1.5,1.5]$} & {$[-1.5,1.5]$} \\
$1.5-1.5-1.5$ & {$[-2.25,2.25]$} & {$[-2.25,2.25]$} \\
$2.0-2.0-2.0$ & {$[-3,3]$} & {$[-3,3]$} \\
$2.5-2.5-2.5$ & {$[-4,3.5]$} & {$[-3.75,3.75]$} \\
$3.0-3.0-3.0$ & {$[-4.8,4.2]$} & {$[-4.5,4.5]$} \\
\hline \hline $0.4-1.6-0.4$ & {$[-1.8,0.6]$} & {$[-1.4,1.0]$} \\
$0.6-2.4-0.6$ & {$[-2.8,0.9]$} & {$[-2.1,1.5]$} \\
$0.8-3.2-0.8$ & {$[-3.6,1.2]$} & {$[-2.8,2.0]$} \\
$1.0-4.0-1.0$ & {$[-4.5,1.5]$} & {$[-3.5,2.5]$} \\
\hline \hline
\end{tabular}

(a) Corrector settings for grid scans.

\begin{tabular}{|c|c|c|}
\hline $\begin{array}{l}\text { Solenoid configu- } \\
\text { ration }[\mathrm{T}]\end{array}$ & $\begin{array}{ll}\text { Horiz. correctors } \\
{[\mathrm{A}]}\end{array}$ & $\begin{array}{ll}\text { Vert. } & \text { correctors } \\
{[\mathrm{A}]} & \\
\end{array}$ \\
\hline $0.4-1.6-0.4$ & -0.645 & -0.2 \\
\hline $0.6-2.4-0.6$ & -0.968 & -0.3 \\
\hline $0.8-3.2-0.8$ & -1.290 & -0.4 \\
\hline $1.0-4.0-1.0$ & -1.613 & -0.5 \\
\hline
\end{tabular}

(b) Corrector center of mass settings.

Table 5.5: The corrector settings in the beam tube and center of mass of the measured profiles for the different magnetic field configurations $\left(B_{\text {gun }}-B_{\text {main }}-B_{\text {coll }}\right.$.

70-73) which gives the direction of the scan. The script scans one row after the other in the vertical direction, changing scan direction for each row. The directionality of the scan is important and can cause artifacts in the transverse scan profile.

The script then terminates by resetting all the correctors to 0 (Code Lines: 130-132). The measured data is saved in a text file. Code 5.1 is an example of such a text file, for a measurement that was taken on 14.05.13 at a filament current of $9.25 \mathrm{~A}$, magnetic field of 0.8-3.2-0.8 kG, a cathode potential of $500 \mathrm{~V}$ and a peak current of $73 \mathrm{~mA}$.

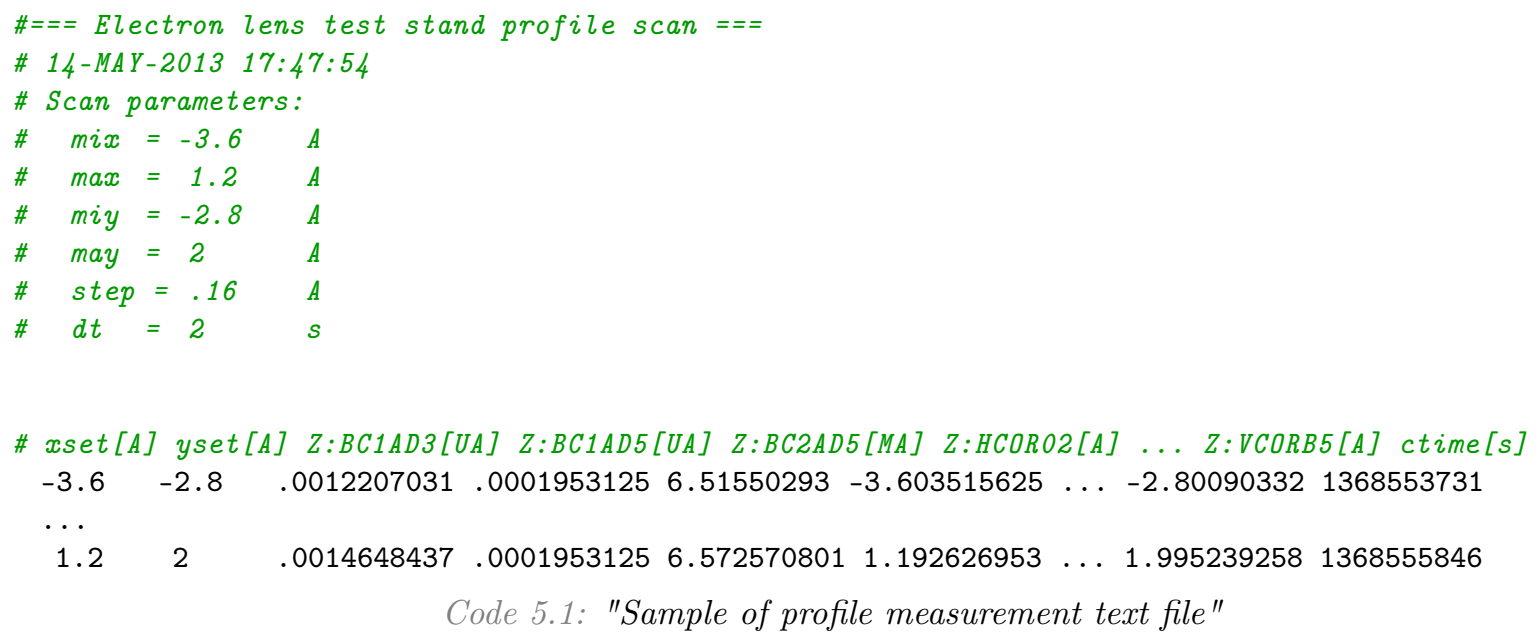

The text file first contains a preamble that lists the settings with which the $A C L$ script was run. In this case we used a $31 \times 31$ grid with a $2 \mathrm{~s}$ measurement per grid point. Following the preamble, the measurements are listed. First the $\mathrm{x}$ and $\mathrm{y}$ positions are given. Followed by the probe current, the tube current and the diode pump vacuum. It then lists the positions of all 6 horizontal and 6 vertical correctors. Lastly it provides the time stamp of the measurement.

\section{R Script}

The full $R$ Script is provided in app. C.1.2.

$\mathrm{R}$ is an open-source GNU's Not Unix (GNU) language for statistical computing and graphical output. It is based on the similar S language and environment which was developed by Bell Laboratories ${ }^{3}$.

The R script starts of with the usual initialization (Code Lines: 8-12), such as the loading of library files, defining very long vectors of all input files, their beam parameters and their transverse offsets. Furthermore some processing and plotting options are set. The script uses two types of color coding

\footnotetext{
${ }^{3}$ www.r-project.org
} 
in contour plots. One is the classical MATLAB color series with blue being the lowest value and red the highest. It was found that this color scheme provided too little contrast for the areas of interest. A second scheme named COLOR. CHART was created to provide more color contrast in the mid-range (Code Line: 175$)$.

The script calculates a few important global quantities such as the electron frequency, flight time, cyclotron and plasma frequency and evolution number (Code Lines: 321-339). These are printed to the standard output. The $\mathrm{R}$ script can also handle measurements made with previous versions of the above mentioned $A C L$ script. The new $A C L$ script provides vacuum data. The $\mathrm{R}$ script thus extracts the data if necessary. The measured data is then stored into 3 arrays: Zprobe, Ztube and Zvac (Code Lines: 421-423). The quantity that is ultimately plotted is Zprobe. The background of the measurement is calculated by averaging the fourth lowest quantile of the measurements. It is then subtracted from the measurements. A fake signal often arises in the bottom left corner. This is removed. Next the measurements are normalized and the center of mass of the profiles are calculated. At this point, the transverse grid is still given in units of amperes, which are provided to the correctors to deviate the beam. To convert them to $\mathrm{mm}$, either the calibration coefficients saved in calx.v and caly.v are used, or they can be recalculated by turning on the option CALIBRATE. CORRECTORS (Code Line: 564). This will ask the user to define the outer and inner beam by defining 8 points on each edge. Given the current deviation and the normal beam size, the calibration coefficients are calculated and printed to the standard output.

The major calculations have now been completed and the script creates several plots of the measurements (Code Line: 681). First a 1D cross-section of the beam, showing the variation of current density along the horizontal direction with the vertical position set to 0 . It then produces $2 \mathrm{D}$ contour plots (Code Line: 720). These come in two variations. One with annotations and one without. Lastly it produces a $3 \mathrm{D}$ plot of the profile (Code Line 800 ).

Having produced all the plots, the script, if the respective options are turned on, outputs; the calibrated profile and a radial plot of current density vs radius from the beam center, which is the average over all azimuthal angles, a particle plot together with a text file containing all the coordinates and if selected a polar decomposition plot (Code Line: 931).

\section{Measured Transverse Profiles}

Profiles were measured throughout the course of this thesis in the TELTS. For a list of all measured profiles, see appendix B.1. Profiles can be sorted by the magnetic fields in the three big solenoids and the cathode voltage. The cathode current is dependent on the cathode voltage through the ChildLangmuir law. In general it was observed that profiles were reproducible when measured with the same parameters. Considering space charge evolution and the angular rotation of the profiles, a chart of all profiles with respect to the magnetic field in the main solenoid and the cathode potential was made. This allows us to uniquely observe profile evolution through these two parameters. Fig. 5.10 depicts such a chart with profiles made after the transmission upgrade mentioned in sec. 5.2. The red lines indicate the expected scaling laws for a constant ratio of pinhole current to total current, which is discussed in section 5.4.3. We can compare this to the profiles that were measured before the transport upgrade, which are depicted in fig. 5.11. It is important to recollect that measurements after the transmission upgrade had a magnetic configuration of $B_{\text {gun }}=B_{\text {coll }}=0.25 B_{\text {main }}$ while measurements before the transmission upgrade had equal magnetic fields in all 3 solenoids. The lack of magnetic compression in the main solenoid has prevented the rapid space charge evolution seen in fig. 5.10 in the measurements made before the transmission upgrade shown in fig. 5.11.

The profiles that are of interest to us are those in the top left corner. Profiles below $0.5 \mathrm{kV}$ tend to become unstable, due to insufficient removal of space charge from the surface of the cathode. At the LHC, the maximum magnetic confinement will be of the order of $6 \mathrm{~T}$, which is the easy attainable magnetic field with today's superconducting magnets. Given this and the assumption that the red lines in fig. 5.10 hold, we see that the profiles in the top left corner are those that represent the profiles that will ultimately scale to LHC parameters. The cathode potential at the LHC will most likely be 


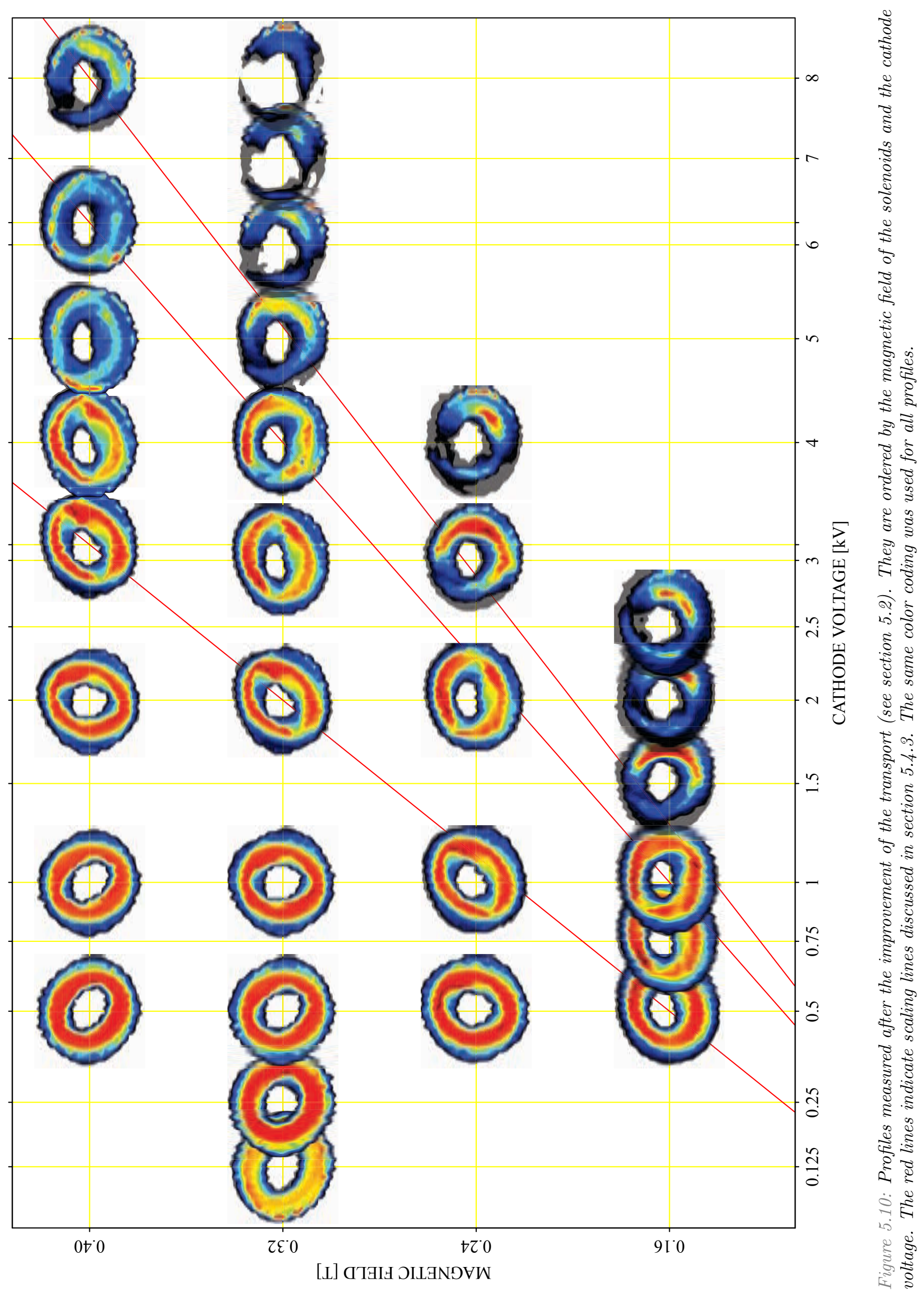




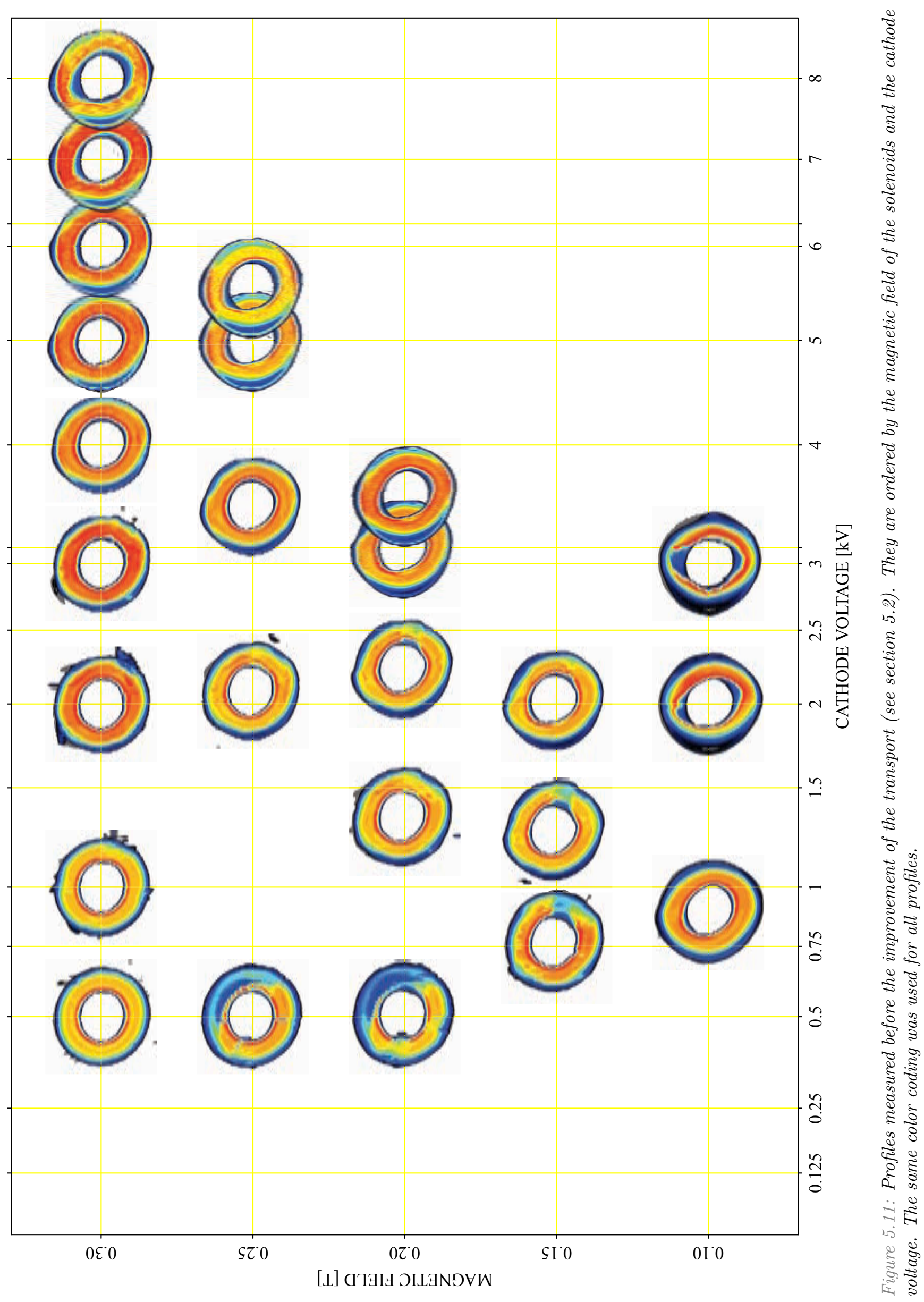




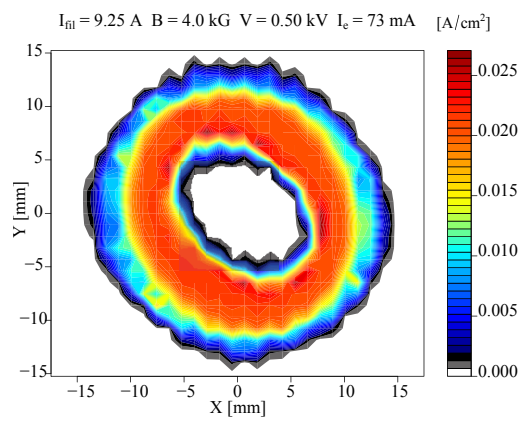

(a)2D beam profile

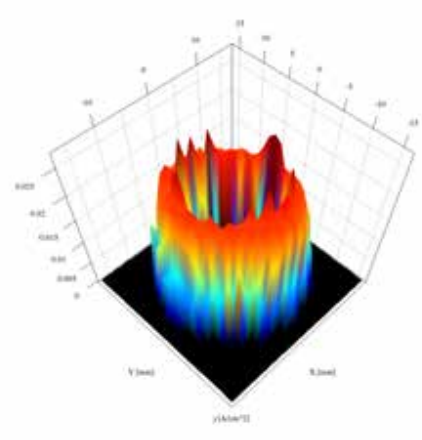

(b)3D beam profile

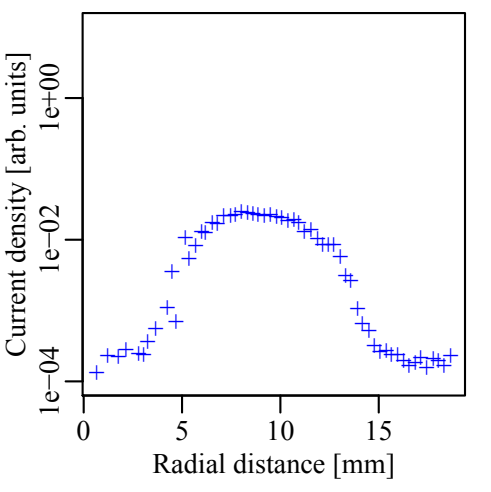

(c)Radial beam profile

Figure 5.12: Beam profile at $B=1-4-1 \mathrm{kG}, \mathrm{V}=500 \mathrm{~V}$ and $I_{\text {peak }}=73 \mathrm{~mA}$. The beam shows a good symmetry, being squeezed a little in the horizontal direction. fig. (b) shows slight slippage through two radially opposing centers. The radial profile in fig. (c) shows a smooth distribution, indicating that the beam is radially similarly distributed for all azimuthal angles.

operated around $4-5 \mathrm{kV}$. Fig. 5.12 shows the 2D, 3D and radial profile of an optimal measurement point at $\mathrm{B}=1-4-1 \mathrm{kG}$ and $\mathrm{V}=500 \mathrm{~V}$.

\subsubsection{Beam Evolution}

This section derives an equation for the angular rotation velocity of the transverse profiles. This is done as a sanity check for the evolutions observed in figures 5.10, 5.13 and 5.14.

The e-beam is essentially a non-neutral plasma that exhibits collective behavior. In order for plasmas to be in equilibrium, they always require a restoring (space charge) and a disturbing self-field force. The radial motion is given by (see: $[25$, p. 175])

$$
\ddot{r}=\frac{\omega_{p}^{2}}{2} r \quad \omega_{p}^{2}=\frac{q^{2} n}{\varepsilon_{0} \gamma^{3} m}\left(1-\gamma^{2} f_{e}\right)
$$

Here $r$ is the transverse position of the profile, $\omega_{p}$ is the plasma frequency, $q$ is the elementary charge, $n$ is the particle density, $\varepsilon_{0}$ is the permittivity of vacuum, $\gamma$ is the relativistic factor and $f_{e}$ is the neutralization factor. As discussed earlier, we can assume non-relativity and thus set $\gamma=1$.

In the case of our electron beam, the restoring force is the $E \times B$ twist, where the magnetic field $B$ is applied externally through the solenoids and the electric field $E$ is radially outward, given by the self fields of the space charge in a 2D approximation. Equilibrium between these two forces is given when the beam is matched (beam envelope on straight line). The beam is matched for a oscillation frequency $\omega$ due to the applied focusing force and the space charge force of (see: [25, p. 191]):

$$
\omega^{2}=\omega_{L}^{2}-\frac{\omega_{p}^{2}}{2}=\left(\frac{e B}{2 m_{e}}\right)^{2}-\frac{n e^{2}}{2 \varepsilon_{0} m_{e}}
$$

$\omega_{L}$ is the larmor frequency, $\frac{q B}{2 m_{e}}$. Due to the radial oscillations, particles have a non-zero transverse velocity and thus exhibit larmor oscillations in the axial magnetic field, causing the beam to rotate. The angular rotation velocity $\omega_{r}$ is given by the dioctron frequency $\omega_{D}[33$, p. 298]:

$$
\omega_{r}=\omega_{D}=\frac{\omega_{p}^{2}}{2 \omega_{c}}=\frac{n e}{2 \varepsilon_{0} B}
$$

where $\omega_{c}$ is the cyclotron frequency which is 2 times the larmor frequency. For a full cylindrical electron beam the angular rotation velocity $\omega_{r}$ is independent of the radial position of the particle because the radial electric field rises linearly with the electric field. Considering a Hollow Electron Beam (HEB) 
configuration and including neutralization $f$ and a contribution due to a rotation of the beam induced by the axial electron current and the azimuthal self-magnetic field $\beta_{z}^{2}$, we obtain the radially dependent azimuthal rotation velocity [33, p. 308]:

$$
\omega_{r}=\omega_{D}\left(1-f-\beta_{z}^{2}\right)\left[1-\left(\frac{R_{i}}{r}\right)^{2}\right], \quad \forall R_{i} \leq r \leq R_{o}
$$

where $R_{i}$ is the inner beam radius, $R_{o}$ is the outer beam radius and $r$ is the particle radius. We assume neutralization to be negligible [28, p. 15], $f=0$, and neglect self-magnetic fields, $\beta_{z}^{2}=0$. It is thus possible to deduce the angle through which the radial profile layers evolve by multiplying with the time needed to transverse the electron beam tube. The distance between the cathode and the collector $L$ is $2.86 \mathrm{~m}$ in the test stand. Assuming that the particle velocity $v_{z}$ is constant throughout the beam tube, we can give the angle of rotation by:

$$
\varphi_{r}=\frac{\omega_{D} L}{v_{z}}\left[1-\left(\frac{R_{i}}{r}\right)^{2}\right]=\frac{n_{e} e L}{2 \varepsilon_{0} B v_{z}}\left[1-\left(\frac{R_{i}}{r}\right)^{2}\right], \quad \forall R_{i} \leq r \leq R_{o}
$$

Given electrons, the number density of electrons $n_{e}$ and the transverse charge density $\rho$ of electrons are related by $n_{e}=6.24 \times 10^{18} \rho \mathrm{L}^{-1}$, where $\rho=\frac{I}{v_{z} \pi\left(R_{o}^{2}-R_{i}^{2}\right)}$. Furthermore using equation 5.4, one obtains:

$$
\begin{aligned}
\varphi_{r} & =\frac{I L}{2 \pi \varepsilon_{0} B\left(R_{0}^{2}-R_{i}^{2}\right) v_{z}^{2}}\left[1-\left(\frac{R_{i}}{r}\right)^{2}\right] \\
& =\frac{I L}{2 \pi \varepsilon_{0} B\left(R_{0}^{2}-R_{i}^{2}\right) c^{2} e V_{a}\left(\frac{\left(2 E_{0}+e V_{a}\right)}{\left(E_{0}+e V_{a}\right)^{2}}\right)}\left[1-\left(\frac{R_{i}}{r}\right)^{2}\right], \quad \forall R_{i} \leq r \leq R_{o}
\end{aligned}
$$

where $c$ is the speed of light, $e$ the electron charge and $V_{a}$ the acceleration potential between the anode and the cathode. Given that $\frac{e V_{a}}{E_{0}}<<1$, we can approximate $\frac{2 E_{0}+e V a}{\left(E_{0}+e V_{a}\right)^{2}} \approx \frac{2}{E_{0}}$ and thus write:

$$
\varphi=\frac{I E_{0} L}{2 \pi \varepsilon_{0} B\left(R_{0}^{2}-R_{i}^{2}\right) 2 c^{2} e V_{a}}\left[1-\left(\frac{R_{i}}{r}\right)^{2}\right], \quad \forall R_{i} \leq r \leq R_{o}
$$

Having a dependency upon $B, V_{a}, I$ and $r$ at this point, one can eliminate the dependency on $I$ through the Child-Langmuir law. As perveance, the measured perveance of the test stand in fig. 5.2 of $5.33 \times$ $10^{-6}$ perv was used. Furthermore $E_{0}=8.19 \times 10^{-14} \mathrm{~J}, \varepsilon_{0}=8.85 \times 10^{-12} \mathrm{~A}^{2} \mathrm{~s}^{4} \mathrm{~m}^{-3} \mathrm{~kg}^{-1}, c=3.00 \times 10^{8} \mathrm{~m} \mathrm{~s}^{-1}$ and $e=1.60 \times 10^{-19} \mathrm{C}$. The inner and outer radius must be taken as an average of the beam radii before, during and after the magnetic compression. Given a magnetic compression of a factor 2, the average beam radii are 0.63 times the cathode beam radii. This gives an average outer beam radius of $8.0 \mathrm{~mm}$ and an inner radius of $4.3 \mathrm{~mm}$. Using these values we can thus write:

$$
\begin{aligned}
\varphi & =\frac{P E_{0} L}{4 \pi \varepsilon_{0} c^{2}\left(R_{0}^{2}-R_{i}^{2}\right) e} \times \frac{\sqrt{V_{a}}}{B}\left[1-\left(\frac{R_{i}}{r}\right)^{2}\right] \\
& =2.7 \times 10^{-2} \frac{\sqrt{V_{a}}}{B}\left[1-\left(\frac{R_{i}}{r}\right)^{2}\right], \quad \forall R_{i} \leq r \leq R_{o}
\end{aligned}
$$

If we consider particles halfway between the average inner and outer cathode radius, $r=6.15 \mathrm{~mm}$, the angular rotation velocity is given by:

$$
\varphi=1.4 \times 10^{-2} \frac{\sqrt{V_{a}}}{B}, \quad \forall R_{i} \leq r \leq R_{o}
$$

Table 5.6 gives a list of all angles of rotation in radians due to the dioctron profile evolution for profiles with an acceleration potential of $\mathrm{V}_{a}=1 \mathrm{kV}$ to $8 \mathrm{kV}$ and $\mathrm{B}_{\text {main }}=0.16 \mathrm{~T}$ to $0.4 \mathrm{~T}$. We can use this equation as a sanity check for the beam evolution and beam profiles we have measured. It is important that $B$ in eq. 5.15 is the average magnetic field throughout the beam tube, which is 0.87 times the magnetic field in the main solenoid. 


\begin{tabular}{|l|r|r|r|r|r|r|r|r|}
\hline \hline Angle of rotation [ $\left[{ }^{\circ}\right]$ & \multicolumn{7}{|c|}{ Acceleration Potential [kV] } \\
\hline Magnetic Field [T] & 1 & 2 & 3 & 4 & 5 & 6 & 7 & 8 \\
\hline 0.40 & 72 & 101 & 124 & 143 & 160 & 175 & 189 & 202 \\
\hline 0.32 & 89 & 127 & 155 & 179 & 200 & 219 & 237 & 253 \\
\hline 0.24 & 120 & 169 & 207 & 239 & 267 & 292 & 316 & 337 \\
\hline 0.16 & 179 & 253 & 310 & 358 & 400 & 438 & 473 & 506 \\
\hline \hline
\end{tabular}

Table 5.6: Transverse rotation angles for particles halfway between outer and inner cathode amplitude as a dependence of acceleration potential $V_{a}$ and magnetic field strength in the main solenoid $B_{\text {main }}$

\section{Evolution as a function of $\mathrm{V}$}

The beam evolution is portrayed in fig. 5.13 for a constant magnetic field of $B=0.32 \mathrm{~T}$. With increasing cathode potential, the beam diverges from a uniform distribution $(V=500 \mathrm{~V})$ to a two-island shape $(V=3 \mathrm{kV})$ and eventually into one major peak $(V=8 \mathrm{kV})$. The radial profiles portray an average over all azimuthal angles of the beam profile. At $125 \mathrm{~V}$ and $250 \mathrm{~V}$ a clear beam edge can be seen at a radius of about $3 \mathrm{~mm}$. As the cathode potential increases, this blurs out. At low voltages, the beam seems to be very stable and axis-symmetrical. As expected, the area underneath the radial profile increases, signalizing a higher beam current. The beam profiles rotate in fig. 5.13 with a radially dependent frequency, indicated by the change in beam profile with changing V. Given that $\omega_{0} \propto \sqrt{V_{a}}$, doubling the cathode potential should make the profiles evolve 1.4 times as fast. According to table 5.6, going from $2 \mathrm{kV}$ to $3 \mathrm{kV}$ causes a rotation of $28^{\circ}$. This can be qualitatively confirmed.

\section{Evolution as a function B}

Fig. 5.14 shows the scaling of the transverse profiles with constant cathode potential. Given that $\omega_{0} \propto B^{-1}$, a change of the magnetic field from $3.2 \mathrm{kG}$ to $4 \mathrm{kG}$ means that the evolution should be slower by $20 \%$. According to table 5.6 , transitioning from $B_{\text {main }}=3.2 \mathrm{kG}$ to $2.4 \mathrm{kG}$ should make the profile turn by $42^{\circ}$. This seems to be the case.

\subsubsection{Beam Scaling}

Considering tab. 5.6 and fig. 5.10, we can assume that profiles will be frozen at magnetic fields of a few Tesla. Still we attempt a first quantitative analysis of dynamical similarity between the profiles, by finding an approximate scaling law for equal profiles with respect to $B$ and $V$.

In 1914, Mr. E. Buckingham published a paper on dimensional analysis that is still cited regularly today (see [34]). He discussed several techniques that allow one to draw fundamental, but necessary conclusions just by considering the dimensional units of equations.

Assuming the necessity to describe a system solely based on $n$ physically different quantities, a physically complete relation can be formulated as

$$
f\left(Q_{1}, Q_{2}, \ldots, Q_{n}, r^{1}, r^{2}, \ldots\right)=0
$$

where $r^{1}, r^{2}, \ldots$ signify a number of ratios between dimensionally equal quantities. Assuming that these remain constant, one can reduce the above to

$$
f\left(Q_{1}, Q_{2}, \ldots, Q_{n}\right)=0
$$

which describes the complete system and is called a physical equation. Each such equation can be expressed in the more specific form

$$
\sum M Q_{1}^{b_{1}} Q_{2}^{b_{2}} \ldots Q_{n}^{b_{n}}=0
$$



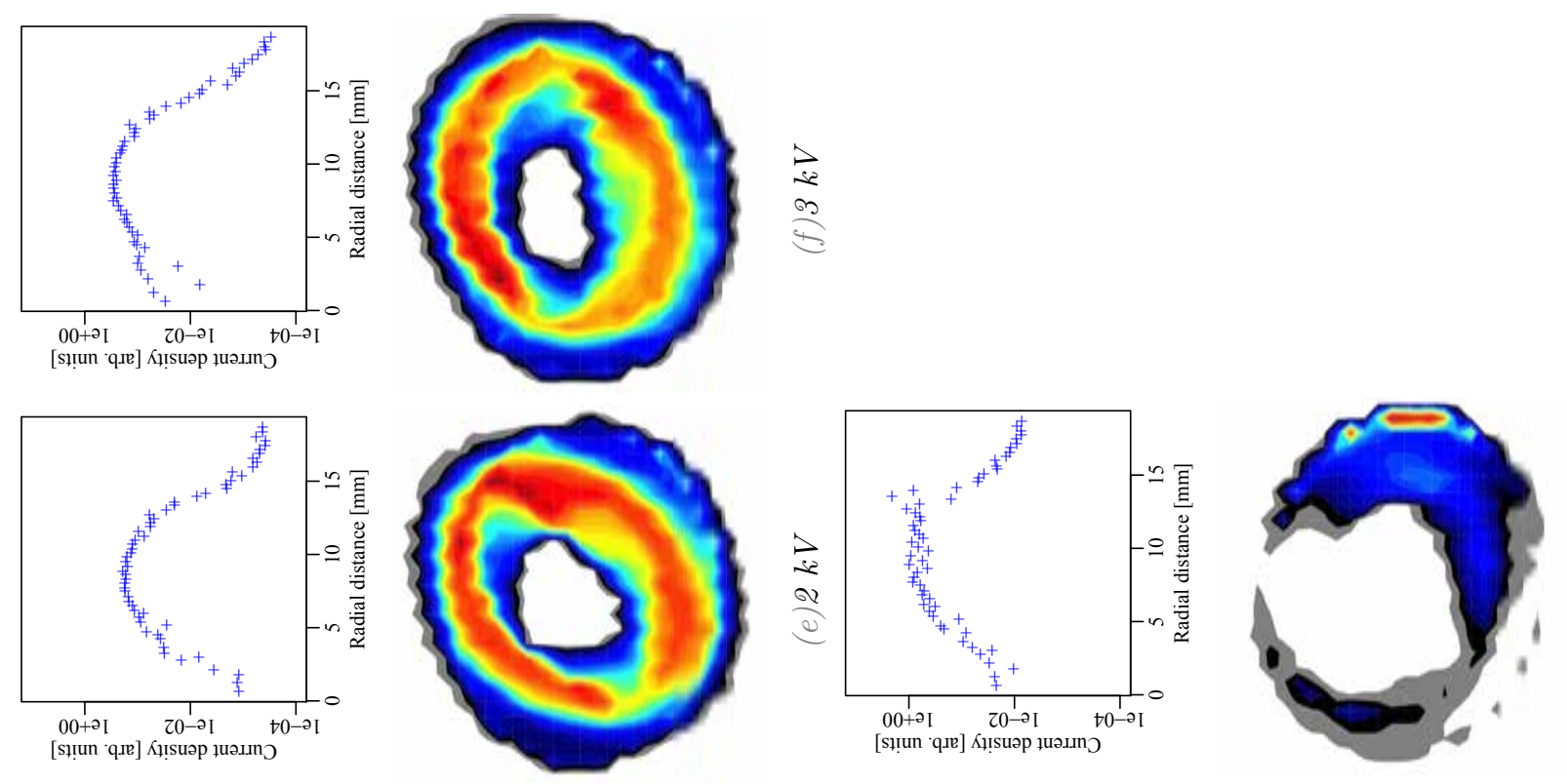

$\stackrel{\infty}{\infty}$
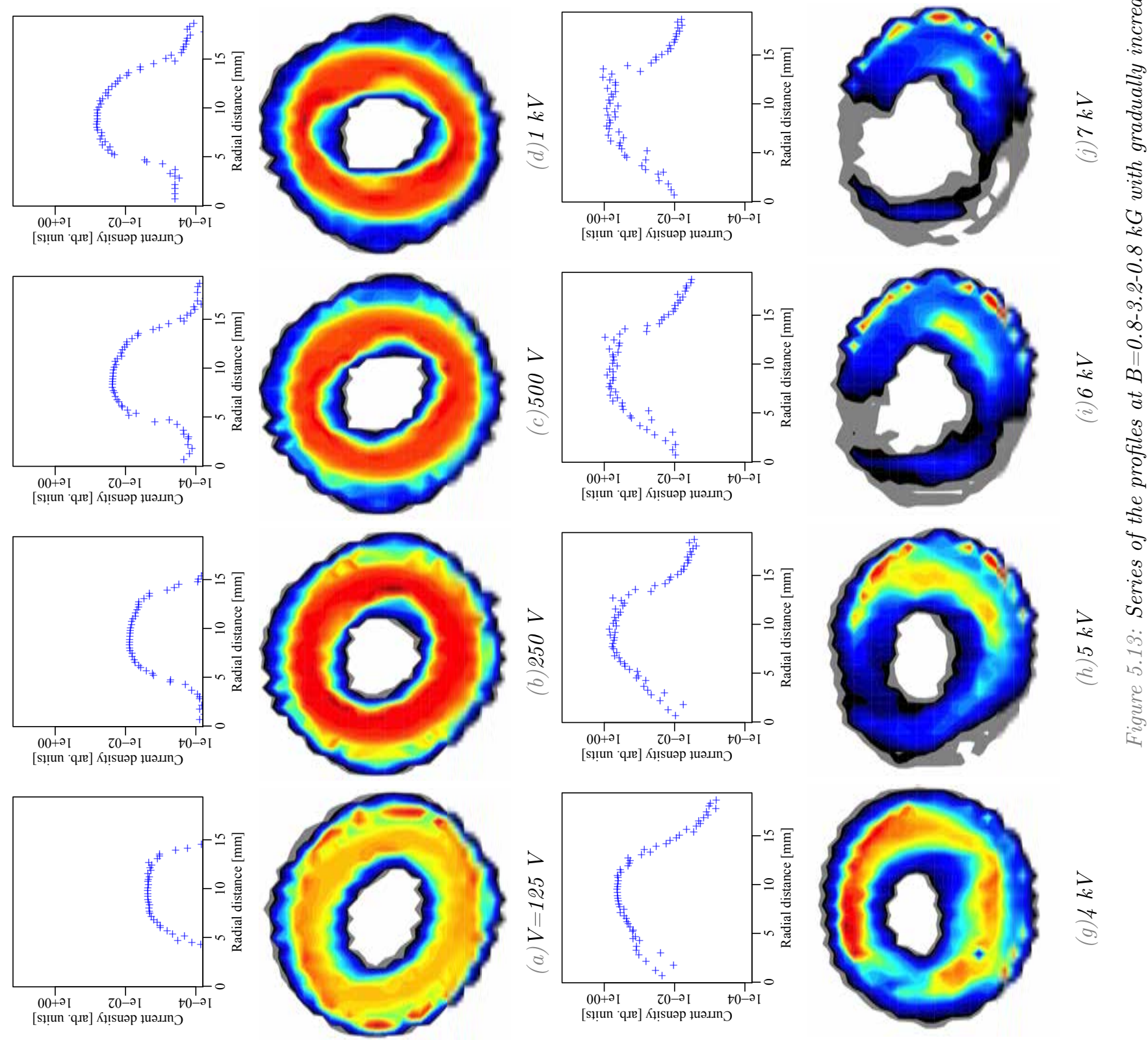

$\frac{3}{2}$
$\frac{2}{2}$ 

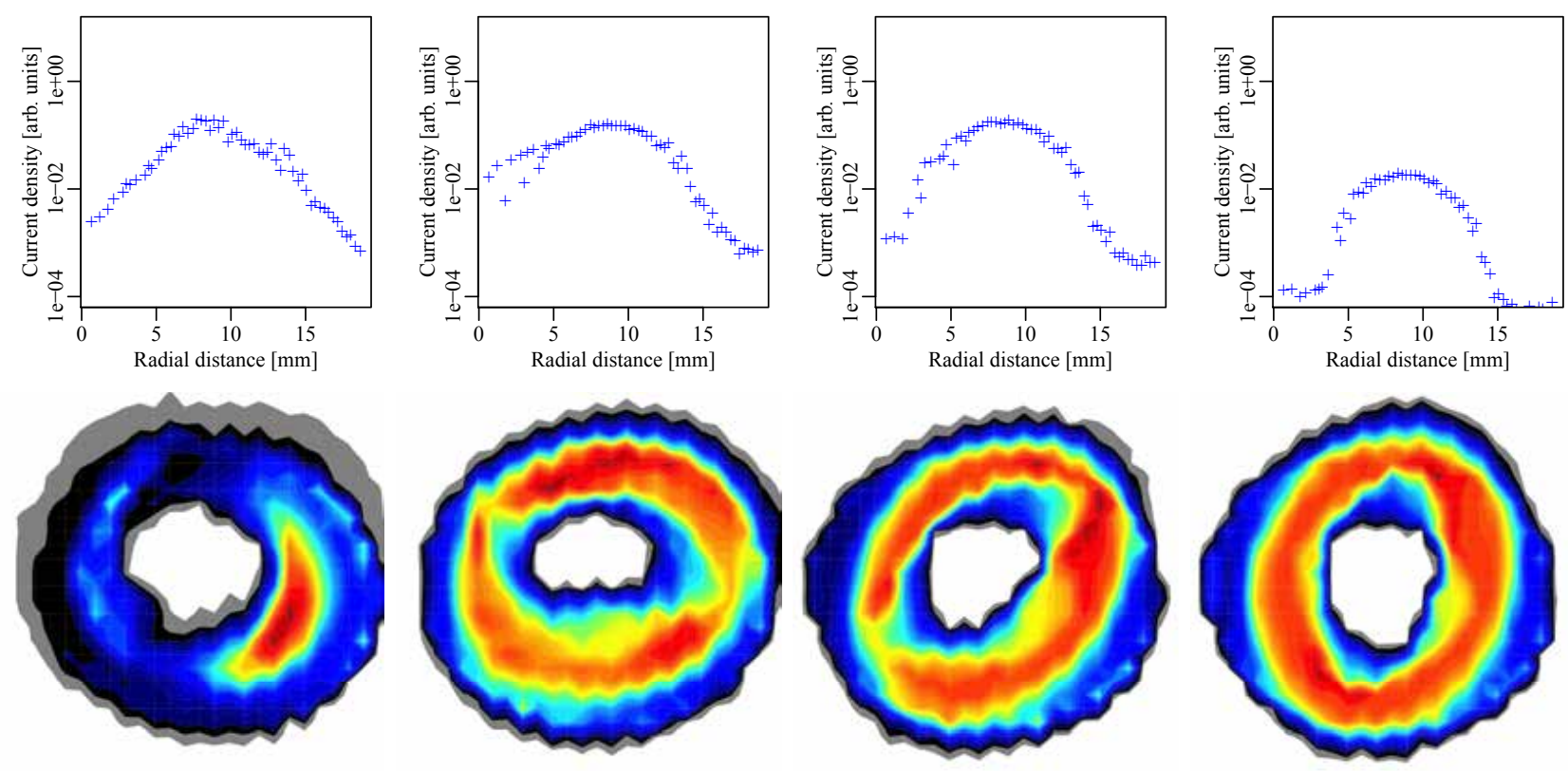

(a) $B_{\text {main }}=1.6 \mathrm{kG}$

(b) $B_{\text {main }}=2.4 \mathrm{kG}$

$V=2 k V$

(c) $B_{\text {main }}=3.2 \mathrm{kG}$
$V=2 \mathrm{kV}$

(d) $B_{\text {main }}=4 k G$ $V=2 k V$

Figure 5.14: Transverse profile series at $V=2 \mathrm{kV}$.

where terms such as logarithms and sin functions have been replaced by their respective expansions. If we now divide through by any one term, this becomes

$$
\sum M Q_{1}^{a_{1}} Q_{2}^{a_{2}} \ldots Q_{n}^{a_{n}}+1=0 .
$$

Each term in this equation must have the same dimension and therefore, one can write the dimensional equations

$$
\left[Q_{1}^{a_{1}} Q_{2}^{a_{2}} \ldots Q_{n}^{a_{n}}\right]=[1] .
$$

By defining the dimensionless products $\Pi=Q_{1}^{a_{1}}, Q_{2}^{a_{2}}, \ldots, Q_{n}^{a_{n}}$ we can rewrite eq. 5.19 as

$$
\sum \mathbb{N} \Pi+1=0
$$

Considering that $\Pi$ is dimensionless, we can rewrite eq. 5.21 as

$$
\sum \mathbb{N} \Pi_{1}^{x_{1}} \ldots \Pi_{i}^{x_{n-k}}+1=0
$$

and thus for every complete physical equation of the form 5.16 we can write a relation in terms of the $\mathrm{n}-\mathrm{k}$ dimensionless quantities $\Pi$, combined from the $\mathrm{n}$ physical quantities $Q_{1}, Q_{2}, \ldots, Q_{n}$ and the $\mathrm{k}$ fundamental units entailed in theses physical quantities, as

$$
\begin{aligned}
\Psi\left(\Pi_{1}, \Pi_{2}, \ldots, \Pi_{n-k}\right) & =0 \\
{\left[\Pi_{1}\right]=\ldots=\left[\Pi_{n-k}\right] } & =[1]
\end{aligned}
$$

Eq. 5.23 describes the system in terms of the $\mathrm{n}-\mathrm{k}$ dimensionless quantities. From this one can then formulate a scaling law.

In order to obtain a perception for the profiles at higher magnetic fields, as used at the LHC, a scaling law for a constant profile evolution was derived using a reduced dimensional analysis, where the permittivity of free space $\varepsilon_{0}$ was omitted. Constant profile evolution requires that the ratio of $I_{\text {pinhole }} I_{\text {total }}$ remains constant. Given the assumption that we operate in the space charge limited regime, we assume Child-Langmuir's law, eq. 4.6, and thus consider the following 5 independent variables 
Given 5 independent physical quantities and 4 SI units, one can define 1 dimensionless parameter that describes the system through the equation

$$
\Psi(\Pi)=R,
$$

where $R=I_{\text {pinhole }} / I_{\text {total }}$. potential $V$, the system lenoth $L$ and the electron mass $e_{m}$ allowing us to write the following according to equation 5.20:

$$
\begin{array}{r}
{\left[B^{\alpha} \times V^{\beta} \times L^{\gamma} \times e_{m}^{\zeta} \times e^{-}\right]=[1]} \\
{\left[\left(\frac{k g}{A \times s^{2}}\right)^{\alpha_{1}}\left(\frac{k g \times m^{2}}{A \times s^{3}}\right)^{\beta_{1}}(m)^{\gamma_{1}}(k g)^{\zeta_{1}}(A \times s)\right]}
\end{array}
$$

The solution of the above equation is: \begin{tabular}{l|rrrr|r} 
Equation & $\alpha$ & $\beta$ & $\gamma$ & $\zeta$ & Dimensionless quantity \\
\hline 1 & 2 & -1 & 2 & -1 & $\frac{B^{2} \times L^{2} \times e^{-}}{V \times e_{m}}$
\end{tabular}

We can thus rewrite eq. 5.25 as

$$
\begin{aligned}
\Psi\left(\frac{B^{2} \times L^{2} \times e^{-}}{V \times e_{m}}\right) & =R \\
\frac{B^{2} \times L^{2} \times e^{-}}{V \times e_{m}} & =\Psi^{-1}(R) \\
B & =\sqrt{V} \times \frac{\sqrt{e_{m}}}{L \times \sqrt{e^{-}}} \Psi^{-1}(R)
\end{aligned}
$$

Considering that $e_{m}$ and $e^{-}$are constant and we do not change the system length, the scaling law thus states that:

$$
B \propto \sqrt{V}
$$

This scaling law coincides with the formula for the angle of rotation in eq. 5.13, obtained from the dioctron frequency $\omega_{D}$. If we wish to determine the scaling for equal profiles, the angle of rotation of the different profiles must be equal. Keeping $\varphi$ constant in eq. 5.13, we obtain the same scaling law of $B \propto \sqrt{V}$.

Having discussed a possible scaling law, fig. 5.15 sorts measured profiles by their magnetic field $B$ in the main solenoid and the scaling factor. The scaling can not be replicated perfectly, but is observable to a given degree. Especially at the lowest scaling factor of $4.47 \times 10^{-3} \mathrm{~T} \mathrm{~V}^{-\frac{1}{2}}$. Here the only profile diverging from the scaling is that at $\mathrm{B}=0.4-1.6-0.4 \mathrm{kG}$ and $\mathrm{V}=500 \mathrm{~V}$. Qualitatively the scaling laws holds, but profiles break down at high cathode potentials, making it difficult to confirm the scaling law. 


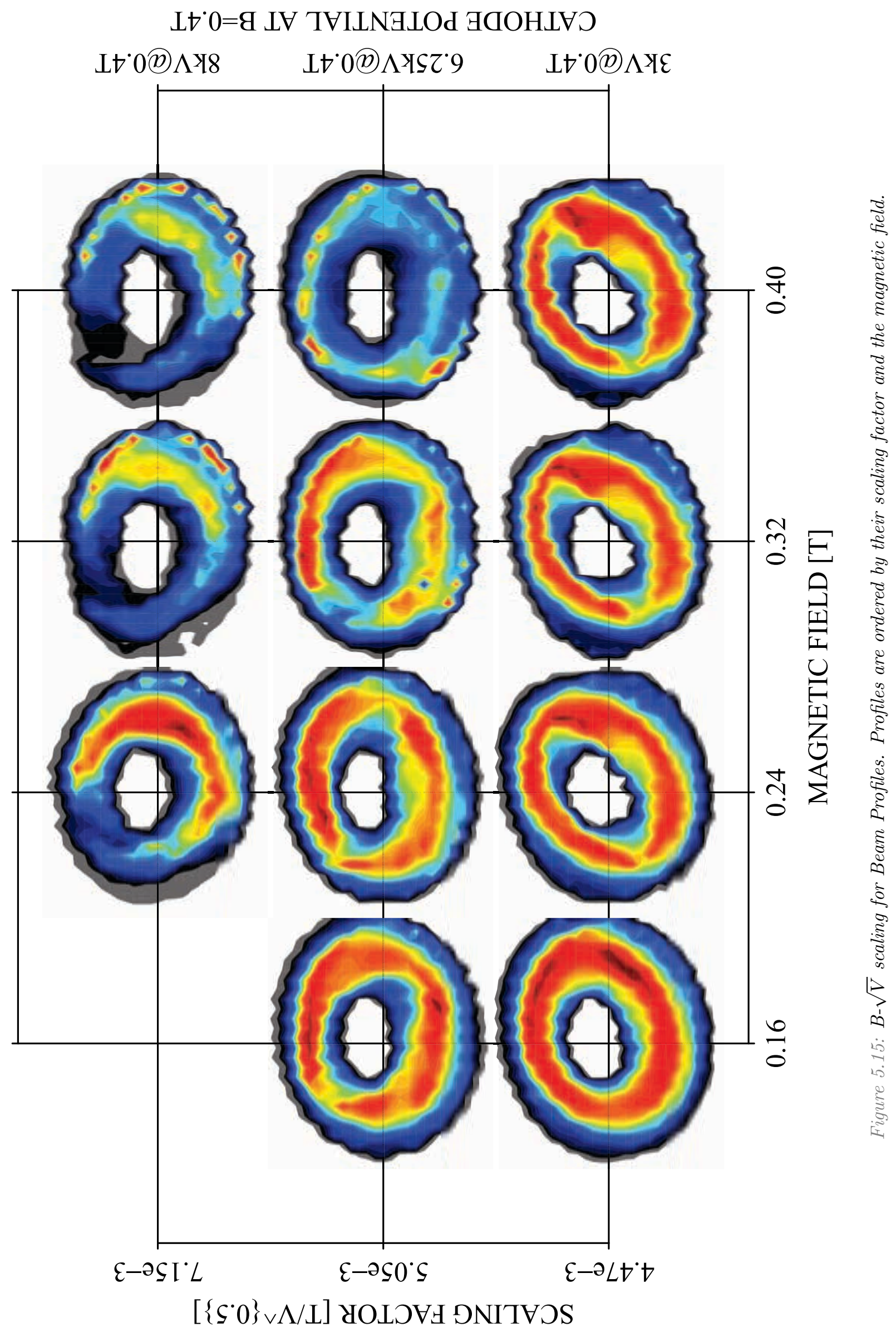




\section{Chapter 6}

\section{Transverse Fields of Measured Profiles}

This chapter discusses the transverse electric fields of the electron beam and an upper limit for the emittance growth due to the electric field of the Hollow Electron Beam (HEB).

Using WARP ${ }^{1}$ the charge density, electric potential and electric field of the measured profiles are calculated. It is important to note that WARP is only being used as a tool for the calculations. It is used in a $2 \mathrm{D}$ setup and no 3D simulations are done. Therefore the detailed discussion of WARP is left to chap. 7, where full 3D simulations are conducted. The transverse fields will be used in tracking studies to examine the effect on the proton beam core and halo. As a preliminary check, the upper limits of the emittance growth due to the transverse electric fields is calculated by assuming the E-field fluctuations in the hole act like random kicks.

WARP was developed in the pursuit of heavy-ion driven inertial confinement fusion. It has been greatly enhanced since and is suited for the simulation of a $H E B$. It contains three coordinate systems: a 3D model (WARP3d) we use in chap. 7; an axis-symmetrical model (WARPrz) and a transverse slice model (WARPxy), which is used here. For further information refer to [35, p. 9]

\subsection{Transverse Field of Hollow Electron Beams}

The field of a $H E B$, can be described using the first Maxwell equation, Gauss' Law in integral and microscopic form.

$$
\oiint_{\partial \Omega} E \cdot \mathrm{d} S=\frac{Q_{\mathrm{encl}, \Omega}}{\varepsilon_{0}}
$$

The left hand side is an integral over the electric field $E$ of the surface of a cylinder $\partial \Omega$. The electric field is assumed to be constant everywhere on the surface $\partial \Omega$. $Q_{\text {encl, } \Omega}$ is the charge enclosed by $\partial \Omega$ and $\varepsilon_{0}$ is the vacuum permittivity. In a $2 \mathrm{D}$ approximation, where the cylinder is assumed to be infinitely long, the front and back parts of the cylinder can be neglected. The right hand side, assuming a uniform line charge distribution, is written as an integral over the charge density $\rho$.

$$
E \cdot 2 \pi r L=\frac{\int_{0}^{R} \rho\left(r^{\prime}\right) 2 \pi r^{\prime} L \mathrm{~d} r^{\prime}}{\varepsilon_{0}}
$$

$r=r(\partial \Omega)$ is defined as the radius at the transverse surface of the cylinder and $L$ as the length of the beam. The electric field $E$ is singled out and some constants are eliminated.

$$
E=\frac{\int_{0}^{R} \rho\left(r^{\prime}\right) r^{\prime} \mathrm{d} r^{\prime}}{\varepsilon_{0} r}
$$

\footnotetext{
${ }^{1} \mathrm{~A}$ particle in cell code developed at Lawrence Berkley National Laboratory.
} 
The inner beam radius is $R_{i}$ and the outer $R_{o}$. Assuming a uniform charge distribution $\rho\left(r^{\prime}\right)=\rho$ in the hollow beam, 3 cases are considered.

$$
\begin{array}{ll}
r \leq R_{o} & E=0 \\
R_{i} \geq r \leq R_{o} & E(r)=\frac{\rho\left(r^{2}-R_{i}^{2}\right)}{2 \varepsilon_{0} r}=\frac{I L\left(r^{2}-R_{i}^{2}\right)}{2 \pi v_{z} \varepsilon_{0} r\left(R_{o}^{2}-R_{i}^{2}\right)} \\
R_{o} \geq r & E(r)=\frac{\rho\left(R_{o}^{2}-R_{i}^{2}\right)}{2 \pi \varepsilon_{0} r}=\frac{I L}{2 \pi v_{z} \varepsilon_{0} r}
\end{array}
$$

These 3 equations fully qualify the electric field of a hollow uniform cylindrical charge distribution. Fig. 2.5 shows the transverse electric field distributions for the profiles corresponding to $\mathrm{V}=500 \mathrm{~V}$ and $\mathrm{V}=8 \mathrm{kV}$ at $\mathrm{B}=1-4-1 \mathrm{kG}\left(B_{\text {gun }}-B_{\text {main }}-B_{\text {coll }}\right)$.

\subsection{Emittance Growth}

From the radial electric field of the beam, the energy of the protons and the length during which the protons are exposed to the electric field, an upper boundary for the additional emittance growth of the proton beam can be estimated. The purpose hereof is to obtain a rough estimate of the emittance growth of the proton beam in the Large Hadron Collider ( $L H C$ ) due to the Hollow Electron Beam Lens (HEBL).

Assuming the transverse electric field to be the only source of a transverse force on the proton particles, the following equation holds:

$$
\frac{\mathrm{d}}{\mathrm{d} t} p_{r}=F_{y}=q \vec{E}_{r}
$$

Here the subscript $r$ indicates the radial direction in the transverse plane, $p$ is the momentum $q$ the elementary charge and $\vec{E}$ the radial electric field. Assuming that the particles are on a perfect design orbit $\left(p_{r}=0\right)$, and that the electric field is constant in time, the above equation can be integrated.

$$
p_{r}=q \vec{E}_{r} t
$$

We define $\theta$ to be the inscribed angle between the total momentum and the axial momentum along $z$. Expressing $p_{r}$ in terms of the total momentum and the momentum along $z, p_{r}=p \sin (\theta)=p_{z} \tan (\theta)$ gives:

$$
\begin{aligned}
p_{z} \tan (\theta) & =q \vec{E}_{r} \\
\rightarrow \tan (\theta) & =\frac{q \vec{E}_{r} t}{p_{z}} \\
\rightarrow \tan (\theta) & =\frac{q \vec{E}_{r} L}{p_{z} v_{z}} \\
\rightarrow \tan (\theta) & =\frac{\gamma E_{0} q \vec{E}_{r} L}{c^{2} p_{z}^{2}} \\
\rightarrow \tan (\theta) & =\frac{E_{\text {tot }} q \vec{E}_{r} L}{E_{\text {tot }}^{2}-E_{0}^{2}}
\end{aligned}
$$

Given that the kick in the transverse direction is $x^{\prime}=\mathrm{d} x / \mathrm{dz}$, it follows that $x^{\prime}=\tan \theta \stackrel{!}{=} \vartheta$. Here $\gamma$ is the relativistic factor, $E_{0}$ is the rest energy of the proton, $v_{0 z}$ is the velocity along $z, q$ is the elementary charge, $L$ is the length of the Hollow Electron Gun (HEG) and $E_{\text {tot }}$ is the total energy of the proton beam. The transverse kick on a particle due to a transverse electric field is thus:

$$
\vartheta=\frac{E_{t o t} q E_{r} L}{E_{t o t}^{2}-E_{0}^{2}}
$$


A normal transfer equation for a particle in an accelerator is given by [36]:

$$
\left(\begin{array}{l}
x_{n+1} \\
x_{n+1}^{\prime}
\end{array}\right)=M\left(\begin{array}{l}
x_{n} \\
x_{n}^{\prime}
\end{array}\right)
$$

where $M$ is the transfer matrix. If the particles are not disturbed by the electron lens, the Courant Snyder invariant (see: [36, p. 105])

$$
\varepsilon=\gamma x_{n+1}^{2}+2 \alpha x_{n+1} x_{n+1}^{\prime}+\beta x_{n+1}^{\prime 2}=\gamma x_{n}^{2}+2 \alpha x_{n} x_{n}^{\prime}+\beta x_{n}^{\prime 2}
$$

is conserved. Considering the kick that is introduced by the electron lens, eq. 6.15 changes to:

$$
\left(\begin{array}{c}
\tilde{x}_{n+1} \\
\tilde{x}_{n+1}^{\prime}
\end{array}\right)=M\left(\begin{array}{l}
x_{n} \\
x_{n}^{\prime}
\end{array}\right)+\left(\begin{array}{l}
0 \\
\vartheta
\end{array}\right)
$$

Assuming that the electron lens is turned on after $n$ turns of the beam, the change of emittance per turn can be found. The emittance after turn $n+1$ is:

$$
\varepsilon=\gamma \tilde{x}_{n+1}^{2}+2 \alpha \tilde{x}_{n+1} \tilde{x}_{n+1}^{\prime}+\beta \tilde{x}_{n+1}^{\prime 2}=\gamma x_{n+1}^{2}+2 \alpha x_{n+1}\left(x_{n+1}^{\prime}+\vartheta\right)+\beta\left(x_{n+1}^{\prime}+\vartheta\right)^{2}
$$

The difference is given by

$$
\Delta \varepsilon=2 \vartheta\left(\alpha x_{n+1}+\beta x_{n+1}^{\prime}\right)+\beta \vartheta^{2}
$$

Given Floquet Transformations (see [37, p.37]), the emittance is retained, but projected onto a circle in a transformed phase space with the axis $x$ and $\alpha x+\beta x^{\prime}$. In this system, the contribution made to the emittance growth by the first term in eq. 6.19 is always orthogonal to the growth and therefore the first term can be neglected. The emittance growth can thus be given as:

$$
\Delta \varepsilon=\beta \vartheta^{2}=\beta\left(\frac{E_{t o t} q E_{r} L}{\left(E_{t o t}^{2}-E_{0}^{2}\right)}\right)^{2} \quad\left[\frac{m}{t u r n}\right]
$$

\subsection{Method and Script}

WARP is a Python package that includes Fortran ${ }^{2}$ in order to facilitate the simulation of particle beams ${ }^{3}$. Scripts are written in Python, using WARP specific syntax. The script used to calculate the transverse fields and the emittance growths (Fieldsolver_single.py) is included in appendix C.2.

After loading the appropriate Python packages and setting options, the code defines a list of all the text files containing the particle positions, produced by profile_analysis.R (see sec. 5.4.1) as well as lists containing the parameters of those profiles, such as the magnetic fields, the cathode potentials and the beam currents. The script has to be executed individually for each profile, setting the profile to be executed through the variable $\mathrm{n}$ (Code Line: 104). It is not recommended to execute several simulations in one run. Upon starting a new run, the charge distribution is not correctly deleted by WARP. One should thus quit Python and restart it between simulations or run Python in a non-interactive mode.

Having defined author information and titles for WARP, it defines the species that is to be loaded; electrons (Code Line: 123). The code then defines the lattice which consists of a drift pipe with a transverse number of mesh cells defined by $\mathrm{nx}$ and ny and a longitudinal mesh size of 1 cell (Code Line: 138-146). The drift pipe is installed as a conductor to enforce the boundary conditions of $\vec{E}_{r}=0$. Consecutively, beam parameters such as the current and the beam energy are set. With all preliminary settings completed, the script now calls the 2D WARP package and generates. The conductor is installed, the particles are loaded and the charge is deposited onto them by loadrho() (Code Line: 202). Particle coordinates are loaded into transverse coordinate vectors xinit and yinit. The particle positions are

\footnotetext{
${ }^{2}$ A programming language specifically suited for numerical computing.

${ }^{3}$ WARP is available at warp. Ibl.gov. For a Willson-Cluster specific WARP, I have compiled a zip-file with installation instructions that can be found here. Tevatron Electron Lens Test Stand (TELTS) wiki page
} 
scaled to the correct positions, in order to accommodate magnetic (de)compression. This scaling is done using $\sqrt{B_{\text {coll }} / B_{\text {main }}}$.

At this point seven types of plots are made: particle plot, charge density plot, electric potential plot, 3 transverse electric field plots and an electric field line plot along the $\mathrm{x}$ and $\mathrm{y}$-axis. Furthermore the script writes the electric fields to a file which has the name _fields.txt appended (Code Line: 262). The transverse fields have thus been completely characterized and the script starts with the determination of the upper limit for the emittance growth.

In order to discuss the emittance growth under expected operating conditions of the $H E B L$ at the LHC, the emittance growth has to be calculated with the inner radius of the electron beam set at $4 \sigma$ and $6 \sigma$ of the LHC beam. $\sigma$ is the standard deviation of the Gaussian distribution of the proton beam in the LHC.

To calculate the $\sigma$ of the LHC, we use the two relations (see [10, p. 66])

$$
\varepsilon_{R M S}=\frac{\sigma_{x}^{2}}{\beta} \quad \varepsilon_{N}=\varepsilon_{R M S} \times \gamma \beta_{c}
$$

where $\varepsilon_{N}$ is the normalized emittance, $\varepsilon_{R M S}$ is the Root Mean Square (RMS) emittance as defined by [38], $\gamma$ and $\beta_{c}$ are the relativistic parameters, $\sigma_{x}$ is the standard deviation from the beam in the $\mathrm{x}$ direction and $\beta$ is the beta-function of the beam at the point of insertion of the HEBL. We set $\beta$ to $250 \mathrm{~m}$ [39]. The normalized emittance is $3.75 \mu \mathrm{m}$ as specified in [7, p. 243]. $\sigma$ is thus given by

$$
\sigma_{x}=\sqrt{\varepsilon_{R M S} \beta}=\sqrt{\frac{\varepsilon_{N} \beta}{\gamma \beta_{c}}}=4.69 \times 10^{-4} \mathrm{~m}
$$

at $\beta=250 \mathrm{~m}, \varepsilon_{N}=3.75 \mu \mathrm{m}$ and $\gamma=4264.39$.

Given $\sigma$ and the cathode radius, the script applies a Gaussian weighting with a standard deviation of $\sigma$ to the measured electric field. This factors in the probability of a particle from the LHC proton beam interacting with a given point of the electric field produced by the $H E B L$. Considering that the transverse profile has been compressed by the magnetic field, $\sqrt{B_{\text {gun }} / B_{\text {main }}}$, the script now selects all points in the $80 \%$ ile of the cathode radius. It then takes the $R M S$ value of all those points and uses them as the transverse field in eq. 6.20. This can be done, because we assume that the electric field in the center acts like random kicks on the core protons and thus only the $R M S$ value of the electric field is significant.

At this stage the transverse field is given at the magnetically compressed cathode radius, $6.75 \times$ $10^{-3} \sqrt{B_{\text {coll }} / B_{\text {main }}}$ and stored in the variable Er_center (Code Line 326). The scaling for the radius goes as $\mathrm{R}^{-1}$ as shown in equation 6.3. The emittance is thus scaled further down to $4 \sigma$ and $6 \sigma$ and stored in the variables Er_center_4 and Er_center_6 (Code Line: 327-328).

Using eq. 6.20, we can thus calculate the emittance growth per turn. In order to obtain the emittance growth in units of $\mathrm{s}^{-1}$, we divide $\Delta \varepsilon$ by the normalized emittance and the revolution time. This quantity is denoted Emittance Growth Rate (EGR) (Code Line: 337. The transverse electric field, emittance growth per turn and $E G R$ are then saved in a table for the cathode radius, and a scaled radius of $4 \sigma$ and $6 \sigma$. It is important to note that this estimate of the emitttance growth is only a rough upper limit, because in reality multipole components will be present in the electric field that do not cause an emittance growth.

\subsection{Results}

Here the general shape of the electric field and the upper limit for the emittance growth are discussed.

\subsubsection{Field Measurements}

Fig. 6.1 shows the plots obtained from WARP for the transverse electric fields of the profile shown in fig. 5.12 at $\mathrm{V}=500 \mathrm{~V}$ and $\mathrm{B}=1-4-1 \mathrm{kG}$. Since the charge density in fig. $6.1 \mathrm{a}$ is symmetric with a slight 
TABLE VI. Initial emittances (wire scan, synch-light, average), emittance growth rates (EGR) $\gamma$, and diffusion slopes $D^{\prime}$.

\begin{tabular}{lcccccccccc}
\hline \hline & \multicolumn{4}{c}{ Emittance $[\mu \mathrm{m}]$} & \multicolumn{2}{c}{ EGR [1/s] } & \multicolumn{3}{c}{ Diffusion $[\mu \mathrm{m} / \mathrm{s}]$} \\
Plane & $\epsilon_{w s}$ & $\delta \epsilon_{w s}$ & $\epsilon_{s l}$ & $\delta \epsilon_{w s}$ & $\epsilon$ & $\delta \epsilon$ & $\gamma$ & $\delta \gamma$ & $D^{\prime}$ & $\delta D^{\prime}$ \\
\hline B1 H & 1.92 & $1.3 \times 10^{-2}$ & 2.15 & $9.6 \times 10^{-3}$ & 2.03 & 0.07 & $5.23 \times 10^{-5}$ & $1.50 \times 10^{-5}$ & $2.49 \times 10^{-8}$ & $7.4 \times 10^{-9}$ \\
B1 V & 1.36 & $2.0 \times 10^{-3}$ & 1.29 & $4.7 \times 10^{-3}$ & 1.33 & 0.02 & $1.70 \times 10^{-5}$ & $1.00 \times 10^{-5}$ & $5.27 \times 10^{-9}$ & $3.2 \times 10^{-9}$ \\
B2 H & 1.69 & $6.6 \times 10^{-3}$ & 2.43 & $1.3 \times 10^{-2}$ & 2.06 & 0.21 & $5.37 \times 10^{-5}$ & $1.90 \times 10^{-5}$ & $2.60 \times 10^{-8}$ & $9.6 \times 10^{-9}$ \\
B2 V & 1.73 & $4.6 \times 10^{-2}$ & 1.98 & $1.2 \times 10^{-2}$ & 1.85 & 0.09 & $1.10 \times 10^{-5}$ & $1.70 \times 10^{-5}$ & $4.79 \times 10^{-9}$ & $7.2 \times 10^{-9}$ \\
\hline \hline
\end{tabular}

Table 6.1: Current EGR at the LHC. Source: [39, p. 8]

\begin{tabular}{|c|c|c|c|c|c|c|c|c|c|c|}
\hline \multicolumn{2}{|c|}{$E G R\left[\times 10^{-5} \frac{1}{s}\right]$} & \multicolumn{9}{|c|}{ Cathode Voltage [kV] } \\
\hline Radius [mm] & $\mathrm{B}_{\max }[\mathrm{kG}]$ & 500 & 1000 & 2000 & 3000 & 4000 & 5000 & 6000 & 7000 & 8000 \\
\hline \multirow{4}{*}{3.325} & 4 & 0.028 & 0.076 & 0.344 & $30.2(3125 \mathrm{~V})$ & 26.6 & 25.6 & $24.5(6250 \mathrm{~V})$ & - & 691 \\
\hline & 3.2 & 0.008 & 0.029 & 9.05 & 15.1 & 22.9 & 276 & 617 & 978 & 1050 \\
\hline & 2.4 & 0.003 & 1.42 & 5.85 & 70.3 & 192 & 318 & - & - & - \\
\hline & 2.4 & 0.414 & 1.40 & 44.7 & - & - & - & - & - & - \\
\hline \multirow{4}{*}{$6 \sigma$} & 4 & 0.039 & 0.109 & 0.495 & $43.4(3125 \mathrm{~V})$ & 38.3 & 36.8 & $35.2(6250 \mathrm{~V})$ & - & 994 \\
\hline & 3.2 & 0.011 & 0.041 & 13.0 & 21.7 & 33.0 & 397 & 887 & 1410 & 1510 \\
\hline & 2.4 & 0.005 & 2.04 & 8.41 & 101 & 276 & - & - & - & - \\
\hline & 2.4 & 0.595 & 2.02 & 64.4 & - & - & - & - & - & - \\
\hline \multirow{4}{*}{$4 \sigma$} & 4 & 0.089 & 0.246 & 1.11 & $97.8(3125 \mathrm{~V})$ & 86.2 & 82.8 & $79.3(6250 \mathrm{~V})$ & 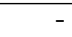 & 2240 \\
\hline & 3.2 & 0.025 & 0.093 & 29.3 & 48.7 & 74.3 & 893 & 2000 & 3170 & 3390 \\
\hline & 2.4 & 0.010 & 4.59 & 18.9 & 227 & 621 & - & - & - & - \\
\hline & 2.4 & 1.34 & 4.54 & 144 & - & - & - & - & - & - \\
\hline
\end{tabular}

Table 6.2: The EGR due to the transverse fields caused by the HEB at the given inner beam radius, magnetic field strength and cathode potential. Green cells give acceptable EGR.

elongation in the y-direction, the resulting electric potential and electric fields are symmetric around the $\mathrm{z}$-axis. The electric field in the center is $\approx 1 \mathrm{kV} \mathrm{m}^{-1}$, as shown in fig. $6.1 \mathrm{~b}$. Comparing this plot to fig. 2.5a, shows that the measured and theoretical electric fields coincide very well. This furthermore indicates that our assumptions in the theoretical deduction in section 6.1 are correct and thus the charge density is near uniform.

In order to contrast these results at a low cathode potential with higher cathode potentials, the plots for $\mathrm{V}=8 \mathrm{kV}$ and $\mathrm{B}=1-4-1 \mathrm{kG}$ are shown in fig. 6.2. The charge distribution in fig. 6.2a is clearly asymmetric. The charge is concentrated in the lower right corner, causing the electric field in the center to be of the order of $\approx 300 \mathrm{kV} \mathrm{m}^{-1}$. A small area of significantly lower electric field, $\leq 100 \mathrm{kV} \mathrm{m}^{-1}$, is apparent in the lower right corner in fig. $6.2 \mathrm{f}$. One would thus expect the proton beam to be much more perturbed at a cathode potential of $-8 \mathrm{kV}$ instead of $-500 \mathrm{~V}$.

\subsubsection{Upper Limit for Emittance growth}

As a reference for the EGR values published on 19 February 2013 in Physical Review Special Topics Accelerators and Beams are used [39, p. 8]. They are presented in tab. 6.1. One should note that the nominal emittance of the LHC of $3.75 \mu \mathrm{m}$ has not yet been reached.

Table 6.2 presents the EGR in units of $10^{-5} \mathrm{~s}^{-1}$. The quantity of $1 \times 10^{-5} \mathrm{~s}^{-1}$ was chosen as an upper limit below which the $E G R$ is acceptable, on the basis that $E G R$ values in table 6.1 are just above that threshold.

When one compares table 6.2 with fig. 5.10, we can see that the $E G R$ tends to rise above $1 \times 10^{-5} \mathrm{~s}^{-1}$ only when the charge distribution deviates strongly from an azimuthally uniform distribution. Values then tend to rise significantly.

Given that the scaling law $B \propto \sqrt{V}$ in section 5.4.3 holds to a certain degree, we are interested in profiles with low cathode potential and high magnetic field. Because the $E G R$ values are only upper boundaries, due to the presence of multipole components, no error calculation was done. 


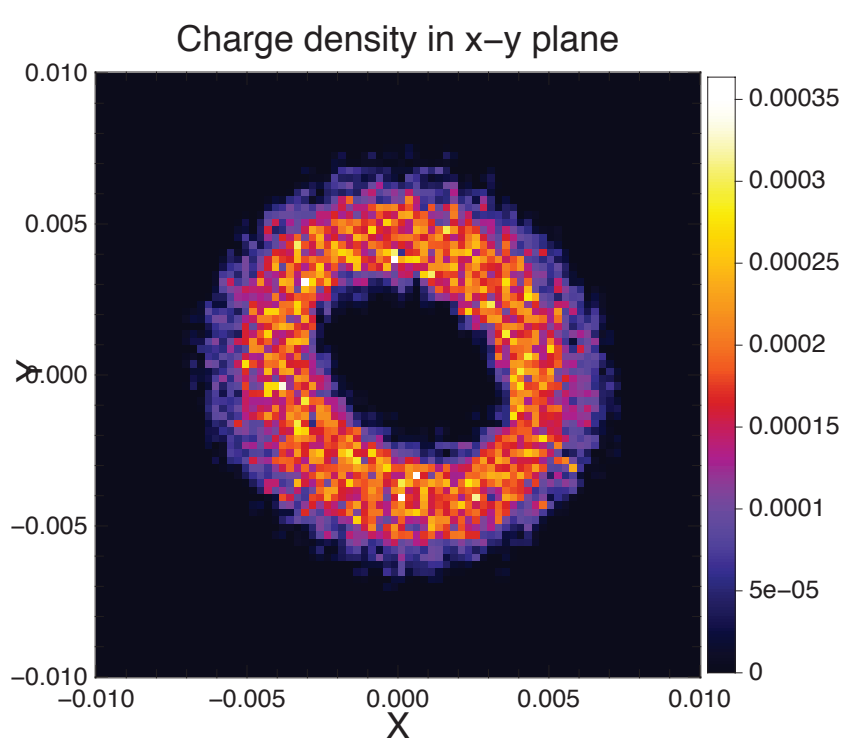

(a) Charge density

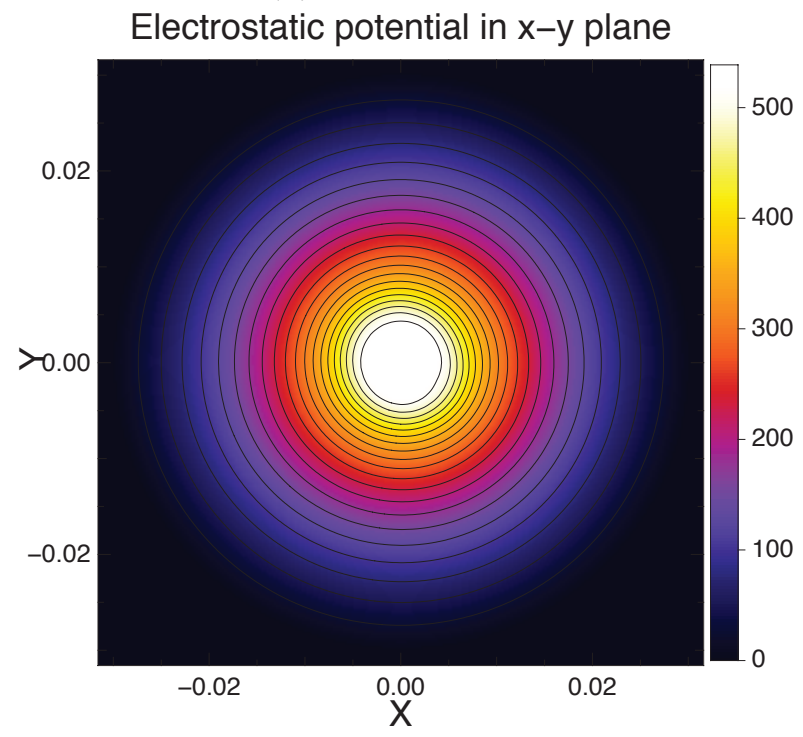

(c)Electric potential

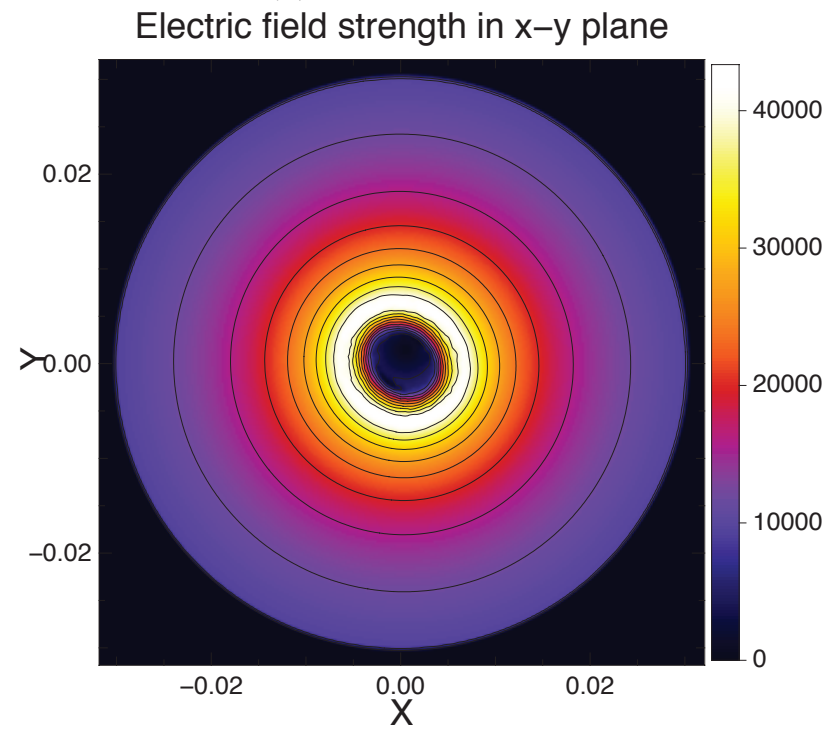

(e)Transverse electric field

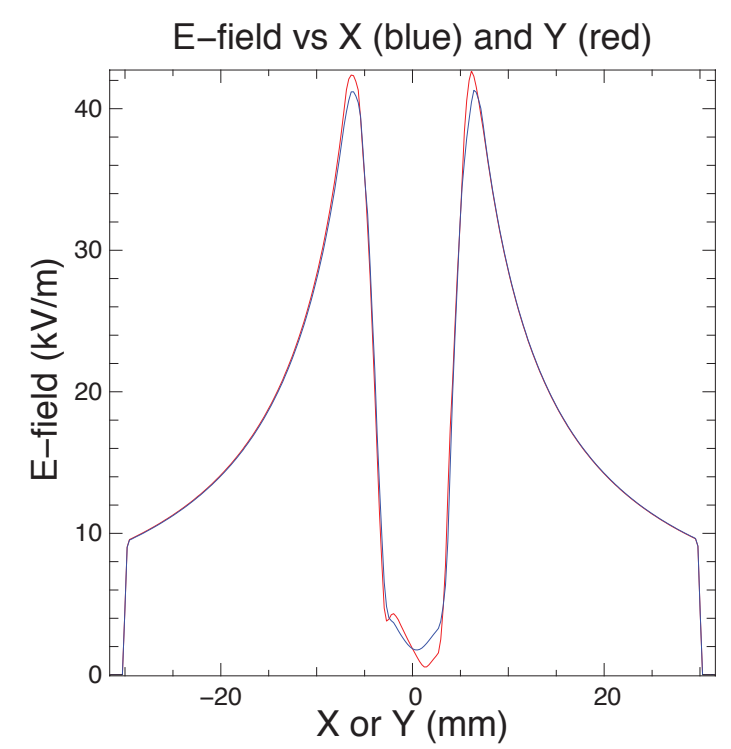

(b) Electric field Line Plot

Electrostatic potential in $\mathrm{x}-\mathrm{y}$ plane

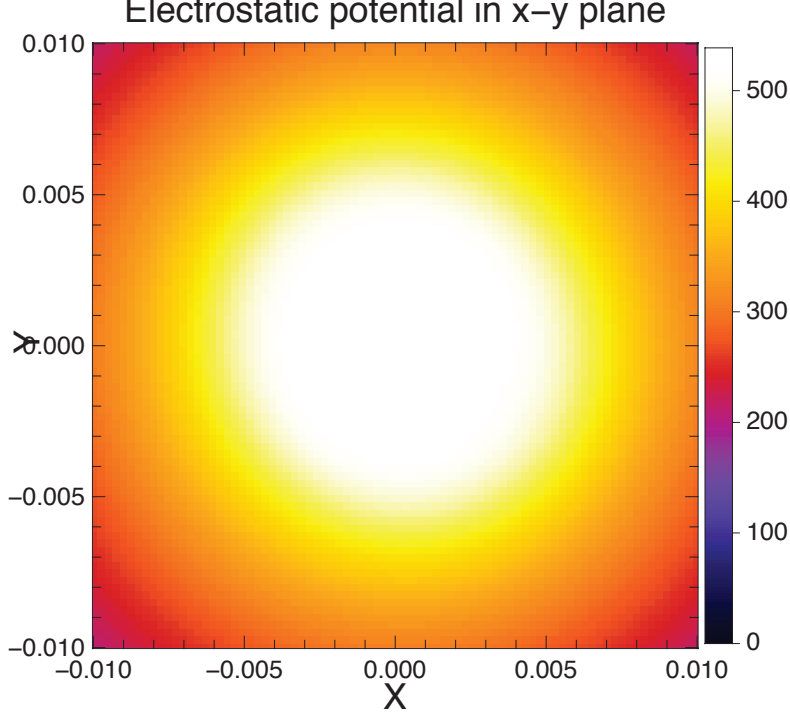

(d)Electric potential in center

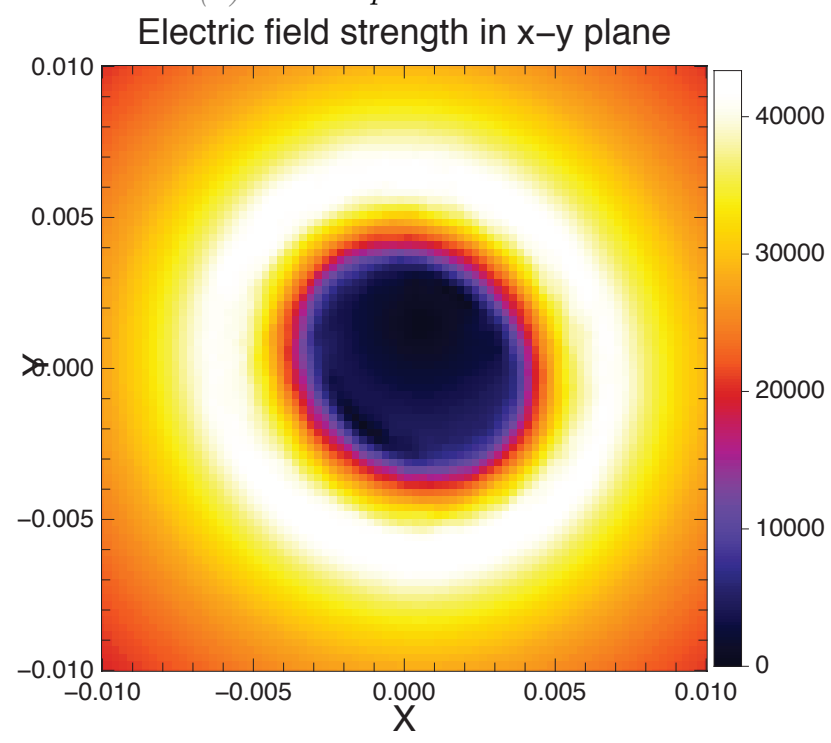

(f) Electric field in center

Figure 6.1: Charge density, electric potential and electric field at $B=1-4-1 k G$ and $V=500 \mathrm{~V}$. 


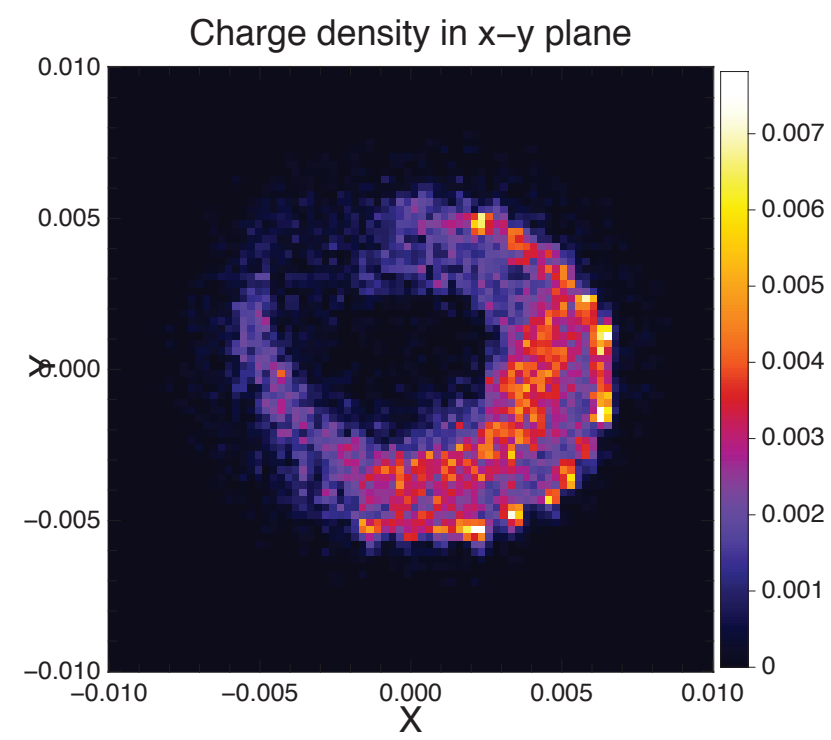

(a) Charge density

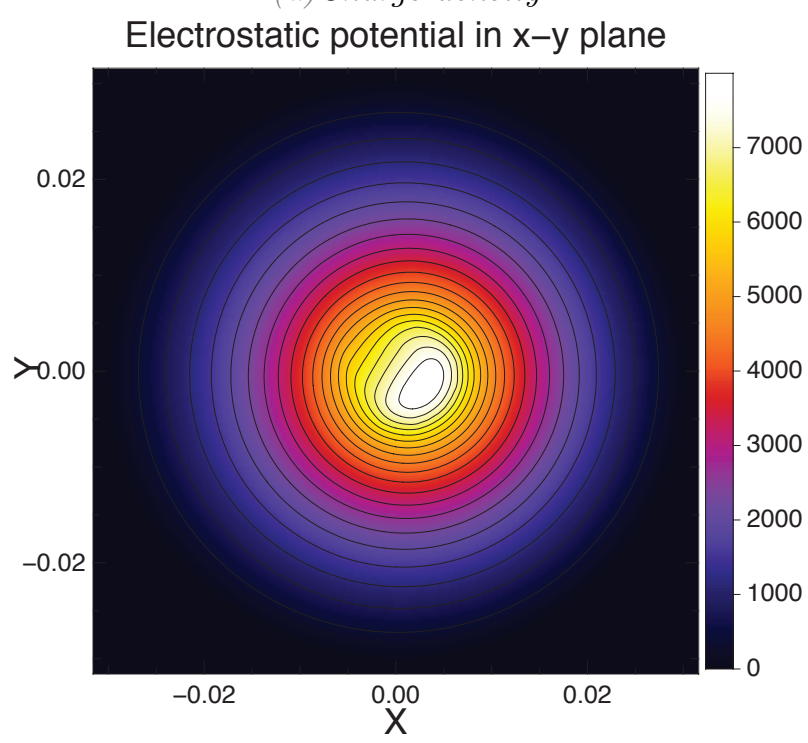

(c)Electric potential

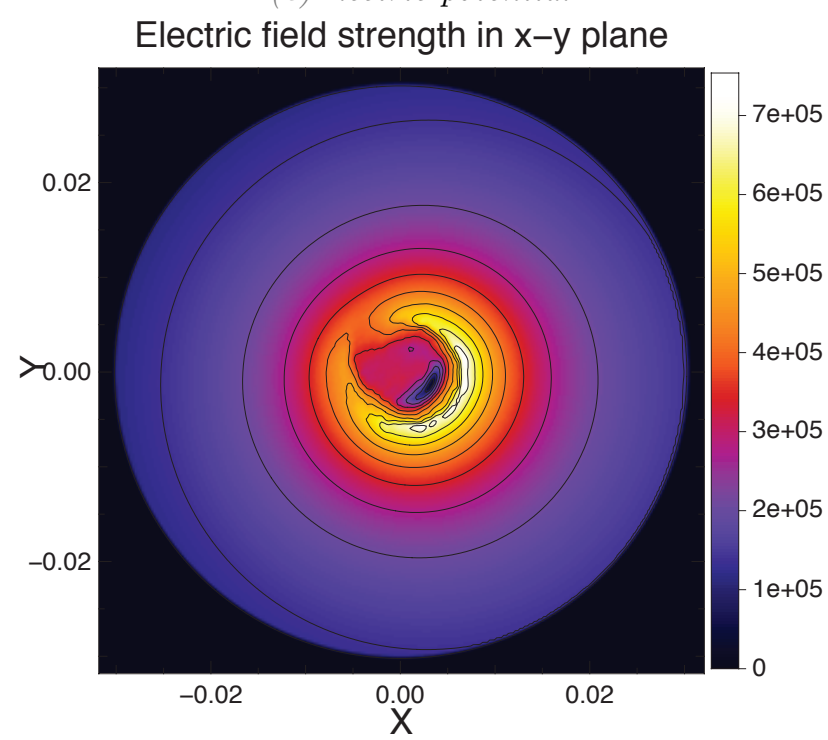

(e)Electric field in y direction

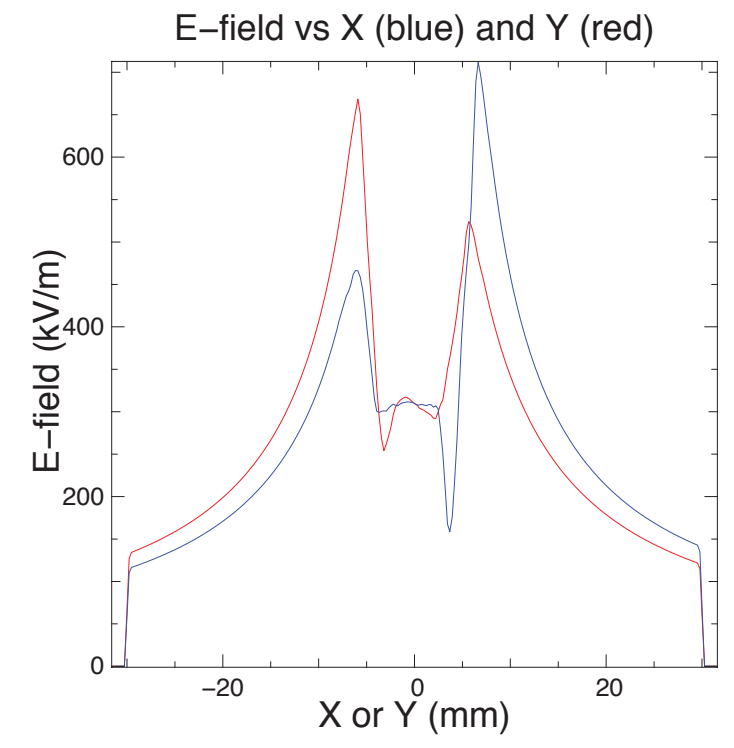

(b) Electric field in $x$ direction

Electrostatic potential in $\mathrm{x}-\mathrm{y}$ plane

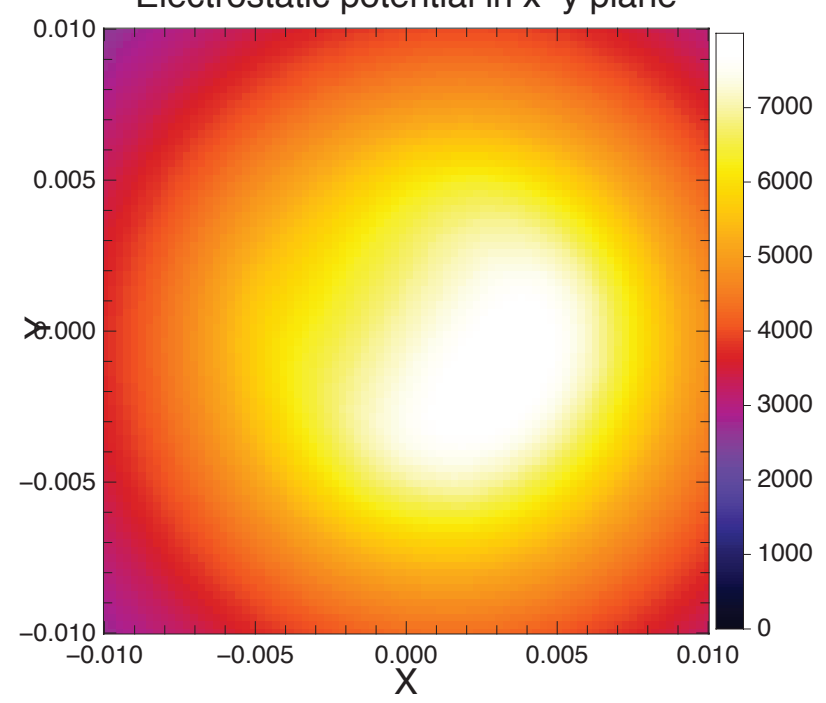

(d)Electric field Line Plot

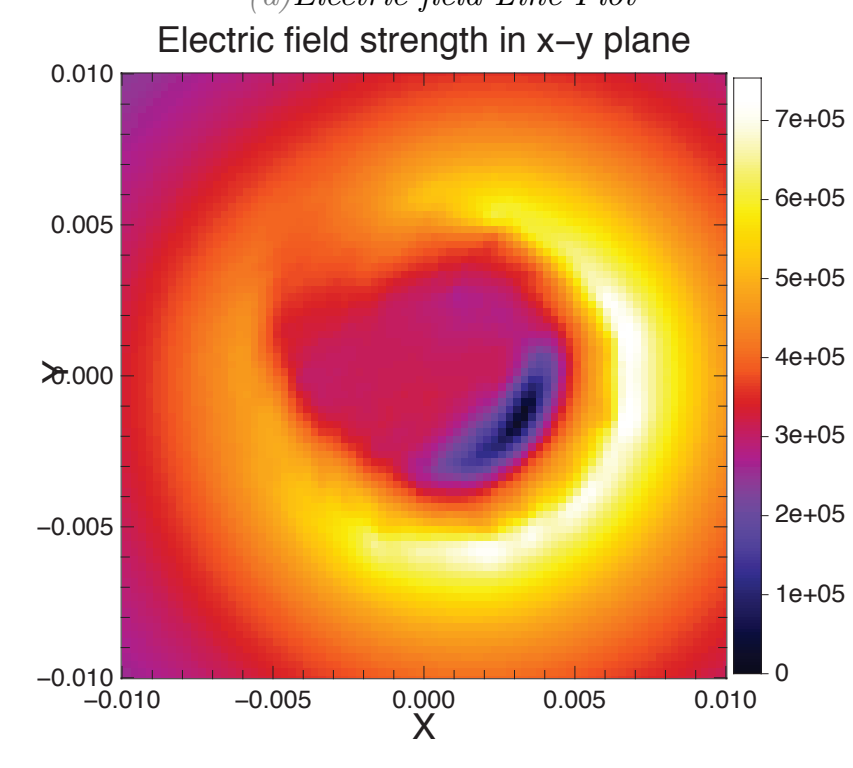

(f)Transverse electric field

Figure 6.2: Charge density, electric potential and electric field at $B=4 k G$ and $V=8 k V$. 


\section{Chapter 7}

\section{Numerical Simulations of Electron Beam Dynamics}

Having discussed all experimental measurements, the first attempt of 3D numerical simulations of the Hollow Electron Beam Lens (HEBL) through a Particle-In-Cell (PIC) code called WARP ${ }^{1}$ will be discussed. This serves as a basis for future 3D beam simulations in order to implement a non-linear map for core and halo tracking studies in SixTrack or Lifetrack. Additionally such simulations are promising for calculating the 3D magnetic and electric fields for the electron lens and for examining the effect of the bends in Tevatron Electron Lens 2 (TEL2) on the beam core. During this thesis, WARP was installed on the Accelerator Simulations Cluster $(T E V)^{2}$ and the first full simulations were completed.

WARP was developed at Lawrence Berkeley National Laboratory in California, USA, by Dave Grote $^{3}$, Jean-Luc Vay ${ }^{4}$ and Alex Friedman ${ }^{5}$. WARP is a PIC code that was designed in order to simulate high current particle beams. It runs in the highly interactive Python interpreter, using precompiled packages. The interpreter allows one to control the simulations interactively.

\subsection{Introduction to WARP}

WARP was installed on the TEV at Fermi National Accelerator Lab (Fermilab). Version-compatibility issues with Python installed on the cluster prevented the installation of WARP. Therefore Python was installed in the home directory with the necessary packages. This new installation was used to compile WARP. Installation instructions and files can be found on the Electron Lens Wiki ${ }^{6}$. Future users of WARP on the TEV may choose to link to my local installation of WARP instead of reinstalling. For this the \$PATHs must be adapted as explained in the instructions on the Wiki.

During this thesis, WARP codes were developed on local machines using a low number of iterations. Normal laptops were mostly sufficient for this. Full simulations were run on the TEV at Fermilab. The TEV cluster has 32 AMD nodes and 12 Intel nodes. Each AMD node has 32 processors and 64 GB of RAM. The WARP simulations are highly memory intensive. In order to run a full simulation, 12 nodes were used with 2 processes per node. This allowed us to use 24 parallel processors and 768 GB of memory. At lower amounts of memory, the code crashed due to heavy disk swapping. This is an issue that will have to be investigated but could not be done in the scope of this thesis.

As mentioned in chapter 6, WARP contains several basic models, including a transverse slice model (WARPxy) and an axis symmetric model (WARPrz). Models exist in 2D and 3D. Additionally, three coordinate frames are available. The lab frame in which all transverse positions are given. In this frame,

\footnotetext{
$1_{\text {warp. Ibl.gov }}$

$2_{\text {tev.fnal.gov }}$

${ }^{3}$ DPGrote@lbl.gov

4 JLVay@lbl.gov

${ }^{5}$ AFriedman@lbl.gov

${ }^{6}$ https : //cdcvs. fnal.gov/redmine/documents/619
} 
the user sets a 0-point and the axial-coordinate increases continually along the axial position. The lab frame follows bends when they are used. In closed accelerators, the lab frame axial coordinate will increase lap by lap. The beam frame and grid frame move along with the beam. The location of the beam frame is given by zbeam.

PIC codes are well known from plasma physics and are used to simulate neutral and non-neutral plasmas. In a PIC code, macromolecules are assigned to cells on a discrete mesh and advanced using the Lorentz equation of motion. Often such codes are also called "Nearest-Grid-Point Particle Mesh models". From the distribution of the macromolecules on the mesh, the charge density is calculated, using a linear interpolation of the particles on the mesh. By solving Poisson's equation, the electrostatic potential and ultimately the electric field are calculated. These calculations are done for each time step, after which the velocities and positions are updated. As explained in [40, p. 28], while positions are usually updated during each full time step, the velocities of the particles are updated every half time step. This can cause errors in diagnostics. Whenever a diagnostic is made in WARP, the isochronous leap frog scheme is used, which additionally updates the velocity at the full time step. Else, the usual leap-frog system is used, for which velocities are only updated at half time steps. For a detailed explanation of the time-step layout, refer to [35, Chap. 3].

WARP combines the power of PIC codes with the easy implementation of accelerator lattices. An extensive set of lattice elements are precompiled and included in WARP. These include dipole, quadrupole and sextupole magnets, accelerator cavities and solenoids. Additional elements can easily be implemented through surfaces of revolution. Furthermore WARP includes several diagnostics packages and allows one to either use a set of precompiled plotting methods or develop individual plotting methods. These can then be executed at predefined time points during the simulation and interactively after the simulation.

\subsection{General Structure of the WARP Script}

The code used for the WARP simulations is listed in appendix C.3. This section discusses the general layout of a WARP code. We have used two injection methods throughout the simulations. First a direct injection from a replica of the 1 inch Hollow Electron Gun (HG1b) and second an injection of the measured profiles from the test bench at the z-coordinate of the cathode. The injection mechanisms will be discussed more closely in sec. 7.3 and 7.4.

The WARP code initially calls the WARP package. The code is separated into a few sections: file loading, options, headers, variables and the actual scripting, which is divided into further sections. The file loading sets the profile that is to be injected or simulated. In options (Code Line: 115) the injection type is defined in the variable machine_injtype and the cathode emittance method is set in machine_emittype. For gun injection, the cathode emits according to the Child-Langmuir law. For profile injection, it emits at a constant current. WARP requires the user to define a variable called runid, which sets the name of the current run. Additionally one can set some variables that are used as descriptors in the plots and the variable runmaker which defines the author of the run. In the section variables (Code Line: 144), the parameters of all lattice elements and some beam parameters are defined, in order to prevent the user from having to deal with the more complicated WARP codes in the section Script (Code Line: 267).

Having defined the runid and the runmaker, the setup (Code Line: 271) command must be called in order to initialize the graphic output file. The output is saved as Computer Graphics Metafile (CGM). A software called gist ${ }^{7}$ is used to view and manipulate the plots from the $C G M$ files. If profile injection is chosen, the selected measured profile from the test bench ${ }^{8}$ is loaded and saved in an array with the first column being the x-coordinates and the second column the y-coordinates. The number of particles that were loaded is printed to the standard output.

\footnotetext{
${ }^{7}$ Available at warp. Ibl.gov

${ }^{8}$ Profile measurements in the test bench are explained in section 5.4
} 
At this stage the code defines the particle species, in our case electrons are designated elec (Code Line: 303). It also defines the number of particles per macro-particles as sw. It then defines beam parameters such as the beam current, the kinetic energy and the time step used in the simulation. The time step is significant, because it needs to be small enough in order to consider all physical effects, such as the plasma frequency $\omega_{p}{ }^{9}$ and the cyclotron frequency $\omega_{c}{ }^{10}$. For the $H E B L$ simulations, the cyclotron frequency exceeds the plasma frequency by a few orders of magnitude. In the case of a $500 \mathrm{~V}$ beam with a magnetic configuration of $1-4-1 \mathrm{kG}, \omega_{c}=7.0 \times 10^{11} \mathrm{~s}^{-1}$ and $\omega_{p}=4.6 \times 10^{8} \mathrm{~s}^{-1}$. The time step is thus determined by the cyclotron frequency. In order to be certain that we use a small enough time step, it was chosen that

$$
\frac{\omega_{c} \Delta t}{2 \pi}=0.25 \Rightarrow \Delta t=\frac{\pi}{2 \omega_{c}} \approx 1 \times 10^{-12}
$$

The electron velocity is set to 0 by the code and deduced from the electron energy through the command derivqty() (Code Line: 315). Next the code sets whether calculations should be done in a relativistic manner. The thermal velocity of the particles is set to 0 . This should be changed in future versions of this code. Having set all the beam parameters, the number of steps necessary to complete the simulation are calculated via:

$$
n_{\text {steps }}=1.1 \times \frac{L}{v_{e} \Delta t}
$$

Here $L$ is the distance the beam travels from gun to collector, $v_{e}$ is the electron velocity and $\Delta t$ is the time step in eq. 7.1. The factor of 1.1 is used in order to increase the number of steps by $10 \%$ to accommodate for the fact that particles move slower than $v_{e}$ while they are in the gun. Next the code defines the size of the electron beam and where it is to be injected (323-357). Amongst others it defines what type of injection is to be used.

Having set up the particle injection, the code now defines the solenoids. It is important to note that these solenoids do not manifest themselves as conductors in the code, but only provide the necessary magnetic fields. It is not necessary to include the solenoids as conductors, since they are positioned outside the drift tube and thus do not affect the electric field inside of the beam tube and are not involved in scraping. Next the script defines the lab frame mesh. When running the code in parallel mode, it is important that the number of grid cells in each direction exceeds the number of processors used to run the script.

In WARP it is possible to set up diagnostic windows, limiting the number of particles that will be involved in the diagnostics. For example it is possible to set up a window so that only the particles in the first 2 time steps after the cathode are plotted. For further information on diagnostic windows please refer to [35, Chap. 7]. Once diagnostic windows are set, it is important to define how often simulation data should be saved (nhist) and plots are made (itplps, itplfreq and itplalways) (Code Lines: 417-433). One can then choose a couple of precompiled plots. These plots are generally less useful and it is recommended to create ones own plots. For more information on plotting refer to [35, Chap. 7].

Having set up the diagnostics and the mesh, the field solver needs to be chosen. First of all the conductor boundary walls are set to absorb particles. Several field solvers are available with WARP. For more information on the individual field solvers, refer to [35, p. 61]. The field solver used is set by the variable top.fstype (Code Line: 442) which is set to 7, 3D multi-grid solver. The multi-grid solver is an extension of the Successive Over-Relaxation Field Solver (SOR). It uses the finite difference form of Poisson's equation in combination with a relaxation parameter that is given by:

$$
\varphi_{i j k}=\omega \Delta^{2}\left(\frac{\left.\rho_{i j k}\right)}{\varepsilon_{0}}+\frac{\varphi_{i+1 j k}+\varphi_{i-1 j k}}{\Delta x^{2}}+\frac{\varphi_{i j+1 k}+\varphi_{i j-1 k}}{\Delta y^{2}}+\frac{\varphi_{i j k+1}+\varphi_{i j k-1}}{\Delta z^{2}}\right)+(1-\omega) \varphi_{i j k}
$$

$$
\begin{aligned}
{ }^{9} \omega_{p} & =\frac{n q^{2}}{\varepsilon_{0} m} \\
{ }^{10} \omega_{c} & =\frac{q B}{\gamma m}
\end{aligned}
$$




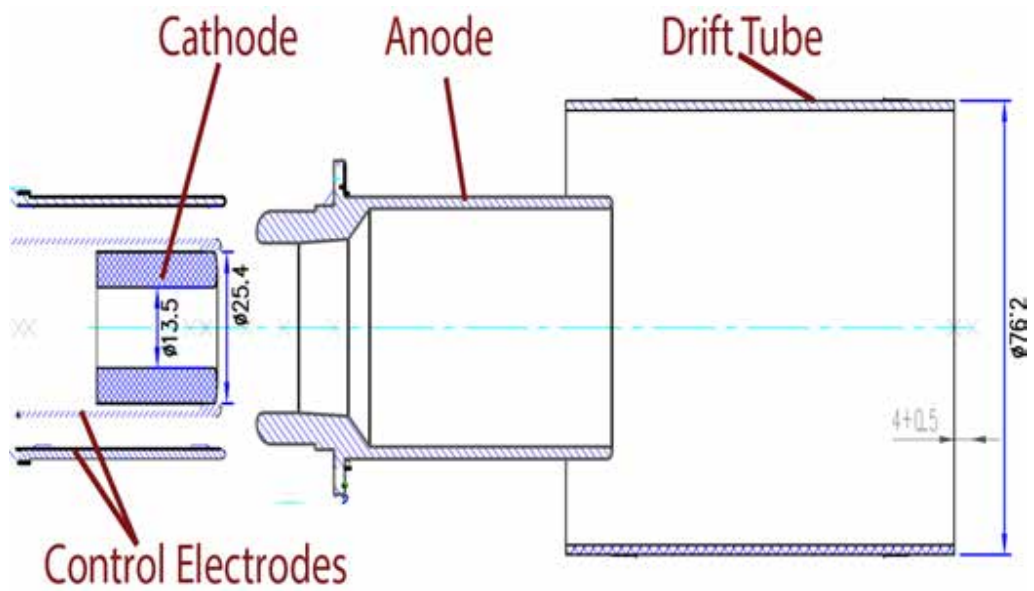

(a)Parts of gun from the technical drawing that are implemented.

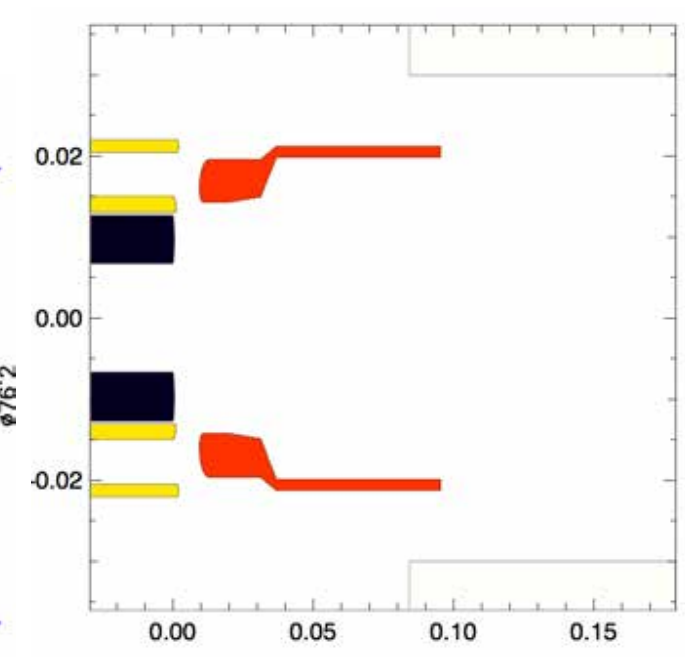

(b) Gun as it is implements in WARP.

Figure 7.1: Technical drawing and plot of the gun that was implemented into WARP.

here $\varphi$ is the potential of the respective cell designated by $i j k, \omega$ is the relaxation parameter, $\rho$ is the charge density and $\frac{1}{\Delta^{2}}=\frac{1}{\Delta x^{2}}+\frac{1}{\Delta y^{2}} \frac{1}{\Delta z^{2}}$ where $\Delta(x, y, z y)$ are the respective grid cell sizes [35, p. 69]. At this point the code is ready to initialize. A package is initialized using the command generate and a time step is made using the command step. The 3D model is initialized (allocating storage, load particles, etc...) using package ("w3d") ; generate() (Code Line: 464)). It is time to implement all the conductors. Both injection methods require the implementation of the drift tube which has a radius of $3 \mathrm{~cm}$. On top of that, the gun injection requires the implementation of all necessary conductors in the gun. The drift tube can be implemented using a precompiled version of a solenoid ZCylinderOut, which defines the outside of a cylinder as a conductor. All conductors that are defined must be installed using installconductors. Lastly self made plots are defined for the diagnostics. Plots that are to be repeated at the time steps given by itplalways and itplseldom must be defined as a function and installed using installplalways and installplseldom (Code Line: 567).

Having completed all the preparatory work, the simulation can now be executed. With the command step one can define how many time steps are to be made. By writing steps (nsteps) (Code Line: 570) the full simulation is done. In order to test new settings, it might be useful to just run a few iteration steps. Lastly a dump of the simulation can be made. This has not yet been implemented, since when running the code on 32 processors in parallel mode, 32 dump files are created, which is counterproductive.

\subsection{Gun Injection}

Gun injection refers to the injection of particles directly of the cathode of the gun. It is thus necessary to implement the gun in the code according to the specifications given in the technical drawings of the gun, fig. 3.7. Fig. 7.1a shows the parts that were implemented and fig. 7.1b shows a plot of the conductors in the WARP code. The most important dimensions of these conductors are listed in appendix D. All 4 conductors were installed using the function ZSrfrv which creates surfaces of revolution. The function takes 3 vectors containing the radial coordinates, the axial coordinates and the radii of curvature for the sections between the specified points. The surface spanned by the points in the vectors is then spun around the lab frame beam axis to produce the conductors in 3D space. The function immediately sets the voltage of the conductors. The conductors must be passed on as a list to the command installconductor in order to be implemented.

On top of installing the gun, the injection of the particles must be specified. This is done earlier in the code in the section labelled injection. First the cathode emission is set to Child-Langmuir law. This is set in the variable top. inject. Additionally the surface from which the particles are injected, their 


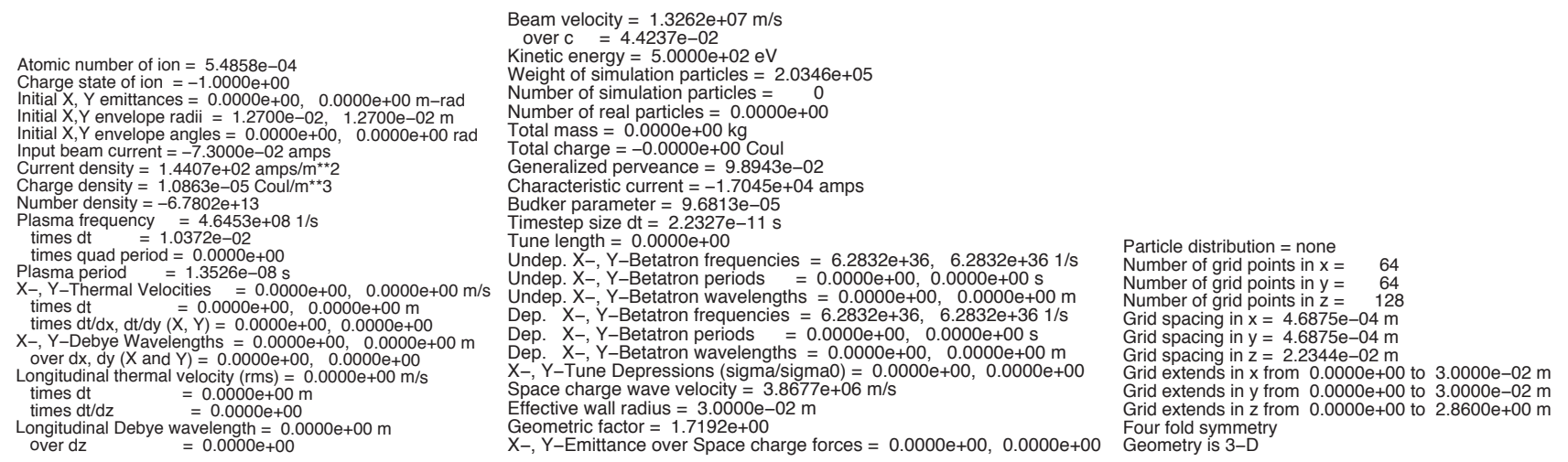

(a)Parts of gun fromt ehcnical drawing that are implemented.

(b) Gun as it is implements in WARP.

(c) Gun as it is implements in WARP.

Figure 7.2: Technical drawing and plot of the gun that was implemented into WARP.

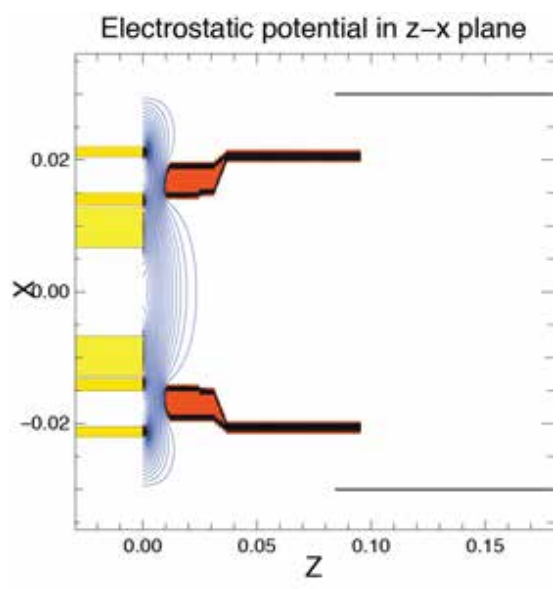

(a)Electric potential of gun.

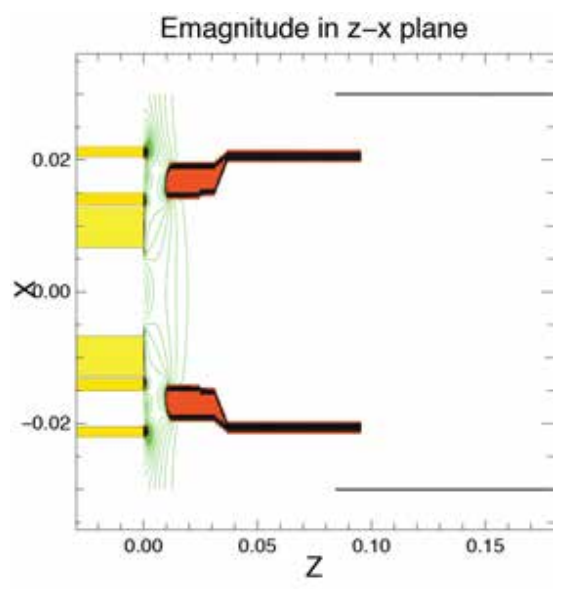

(b) Electric field of gun.

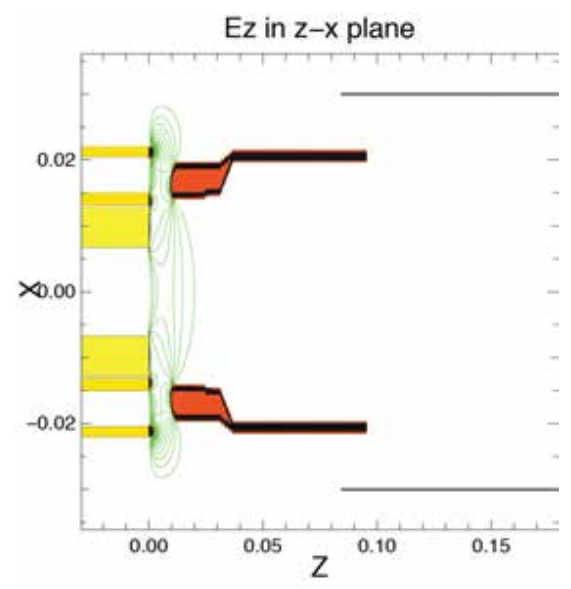

(c) Electric field in z of gun.

Figure 7.3: Plot of the electrostatic potential and electric field of the gun implemented in WARP.

angles and their velocities are defined. Lastly the voltage of the injector source is set with vinject. An additional variable called elec.npinject defines how many macro particles are injected during each time step. In the case of Child-Langmuir law injection, this value may be set to an approximate value, since the law will change the number of particles injected as necessary.

Fig. 7.2 shows the settings used for a normal run on the TEV. The generalized perveance in fig. $7.2 \mathrm{~b}$ serves as a reference to our measured gun perveance. In section 5.3.5 we measured a generalized perveance of $7.93(7) \times 10^{-3}$. The WARP simulation gives us a generalized perveance of $9.89 \times 10^{-2}$. Our measured generalized perveance is $\approx 80 \%$ of our simulated generalized perveance.

The electrostatic potential and electric field of the gun that was implemented in WARP is shown in fig. 7.3. It is important to notice that one needs to increase w3d.nz to a very high number in order to present the field correctly (2048). It is thus recommended to reduce the system length machin_syslen and only simulate the gun for these plots. The plots in fig. 7.3 were made with w3d. $\mathrm{nx}=128, \mathrm{w} 3 \mathrm{~d} \cdot \mathrm{ny}=128, \mathrm{w} 3 \mathrm{~d} \cdot \mathrm{nz}=512$.

Lastly fig. 7.4 shows the full trajectory of the beam. Three main features can be recognized. First, we see the clear magnetic compression of the beam as it enters the main solenoid that has a 4 -fold magnetic field, meaning a 2-fold compression. Additionally we see that the beam is nicely hollow, portrayed through the two yellow bars. And lastly we see some suspected micro-bunching as the beam reaches the end of the beam tube. 


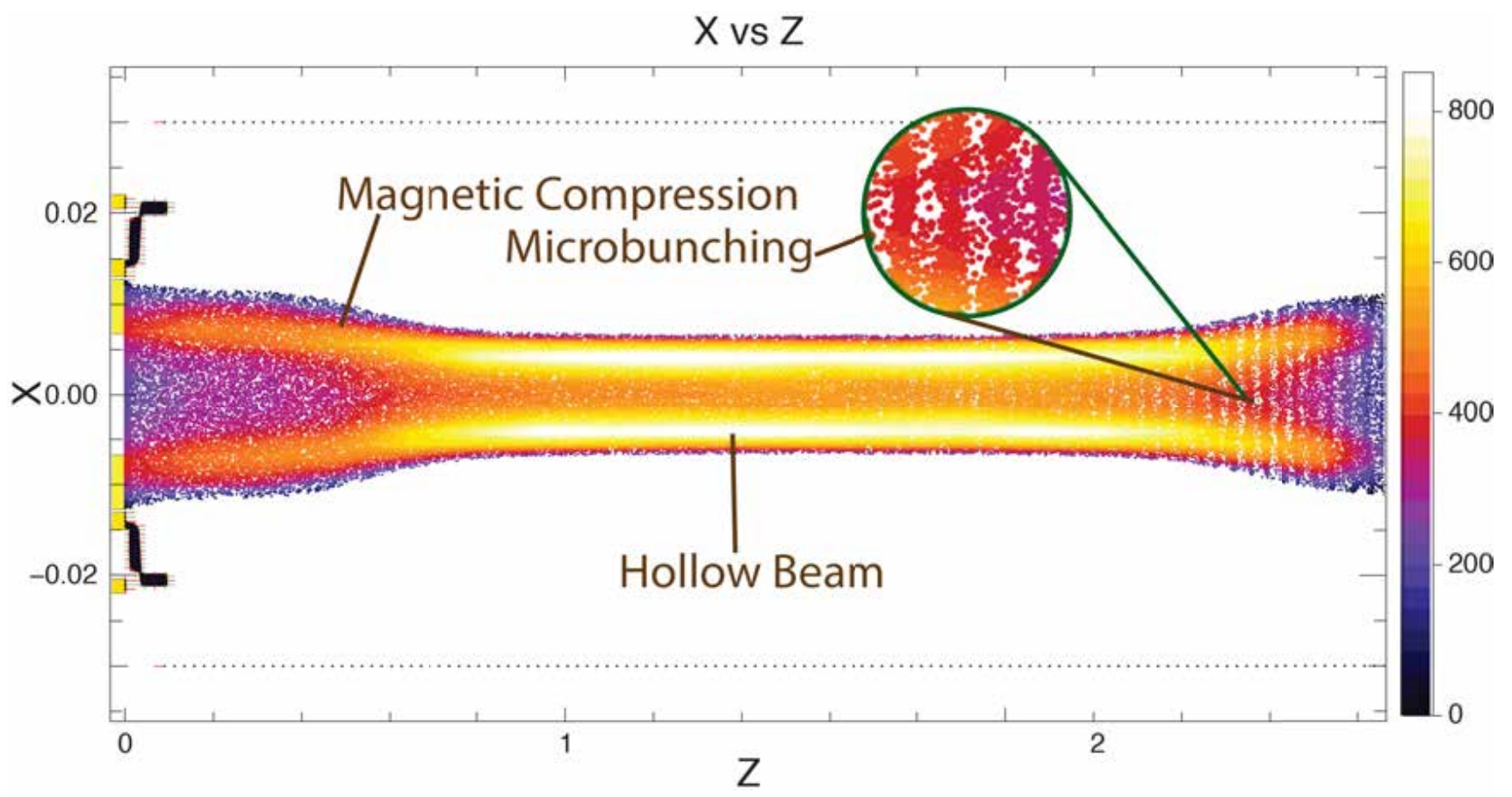

Figure 7.4: Full electron beam as implemented in WARP due to gun injection. Three important features are visible: the magnetic compression of the beam before and after the main solenoid, the hollow center of the beam and micro bunching towards the end of the beam.

\subsection{Profile Injection}

Profile injection refers to the injection of particle distributions measured in the test stand. The assumption is that the measured profile at high magnetic field and low cathode potential has not yet undergone significant space charge evolution (see sec. 5.4.2) and is thus very similar to profiles that are emitted directly from the gun. The measured profile that is used for profile injection is that at a magnetic field of $B=1-4-1 \mathrm{kG}$ and a cathode potential of $-500 \mathrm{~V}$. This measured profile distribution is shown in fig. 7.5.

The injection of this profile is a user defined injection method in which a vector fns passes the path of the profile to the code. The code then imports the particle positions through the command fromfile into a vector posi. The $\mathrm{x}$ and y coordinates are read into one long vector in sequence, requiring one to split the single vector into an array with a column of $\mathrm{x}$ coordinates and a column of y coordinates. This is done using reshape. During profile injection, a test is conducted to verify that the right particles are injected, which is given

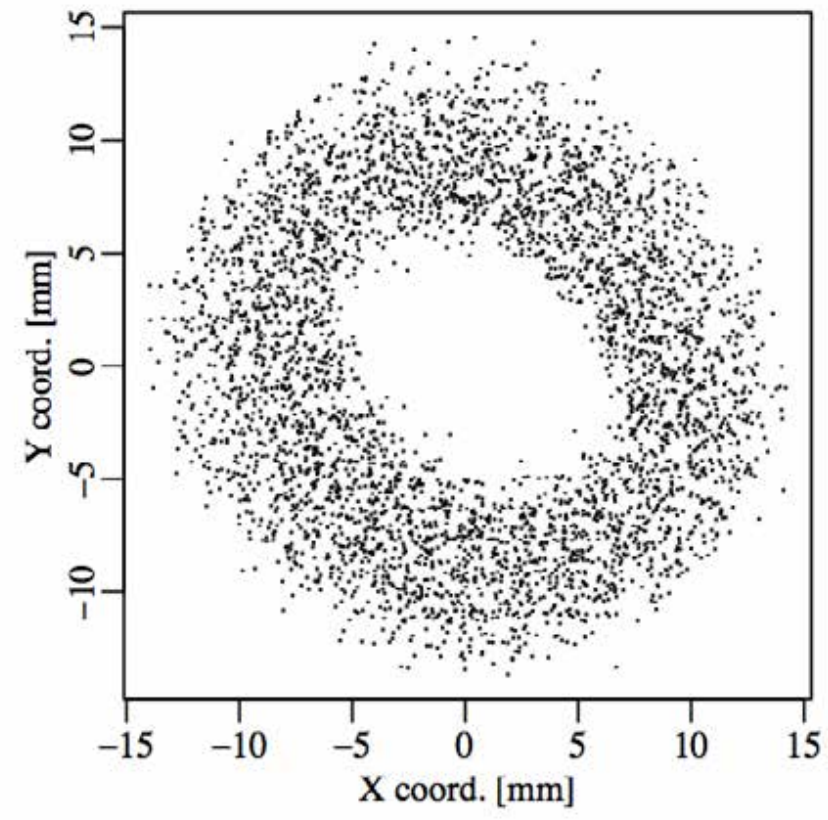

Figure 7.5: Particle distribution of measured profile at $B=1-4-1 k G$ and $V=500 \mathrm{~V}$ through the variable w3d.inj_js, and the number of particles to be injected each time step is defined. Next the coordinates are passed on to w3d.xt, w3d.yt and w3d.zt which define the particle positions in the 3D lab frame. Additionally the initial 


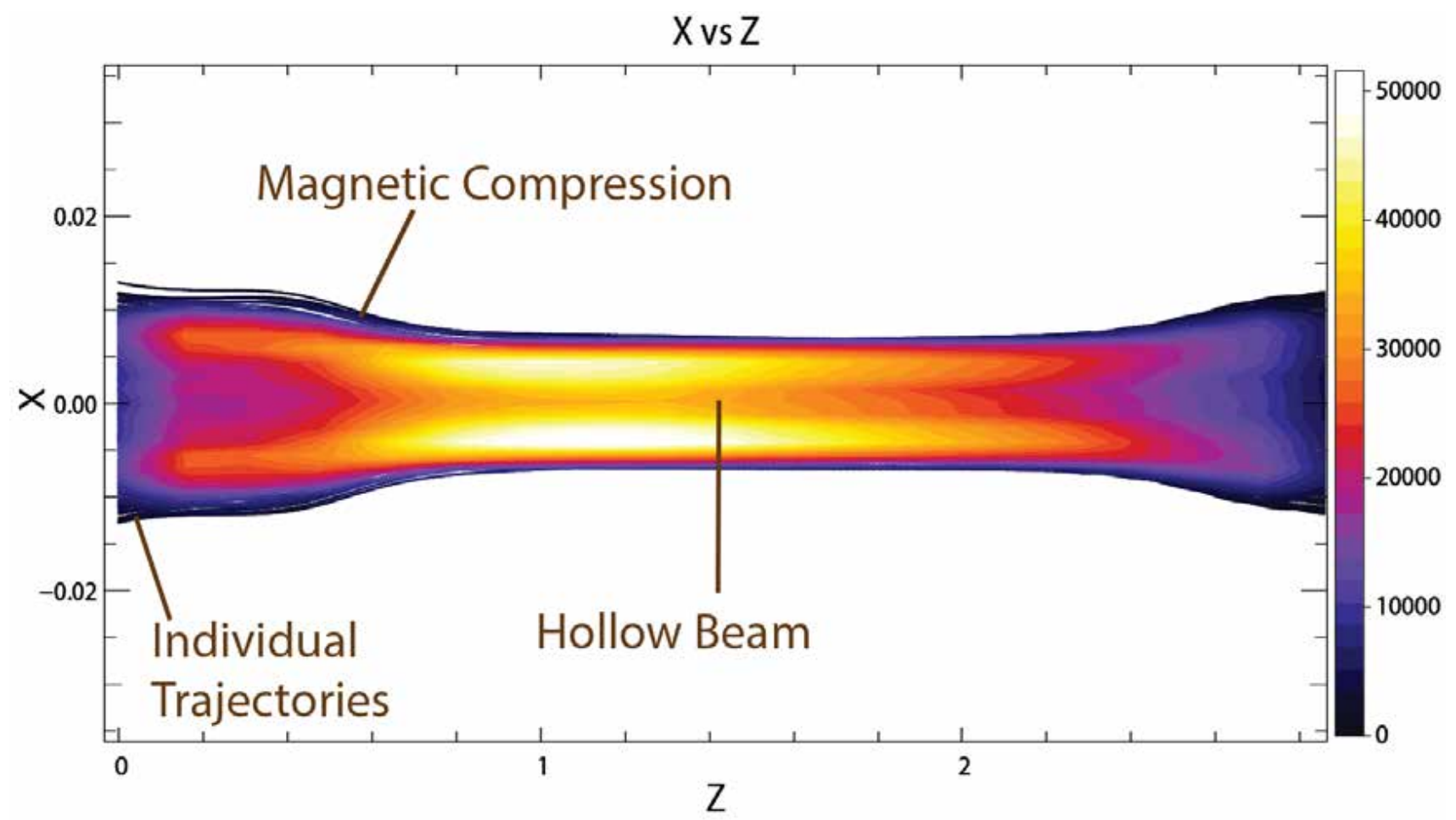

Figure 7.6: Full electron beam as implemented in WARP due to profile injection. The electron density is too high using this injection.

velocities are set to 0 for the transverse velocities and to the beam velocity for the axial velocity. To finalize injection, the source has to be installed using installuserparticlesinjection.

The injection method is not finalized at this stage, but initial runs have been conducted. The main issue with this injection type is that it injects too many particles. This is seen when comparing figure 7.6 with fig. 7.4 , which has $\mathrm{a} \approx 60$ times lower particle count. The high particle count makes it nearly impossible to process these profiles. It is assumed that this is a consequence of the whole loaded profile being injected at each time step. Further development on this injection was not done, since gun injection was considered to be more important.

\subsection{Directions for further studies on 3D WARP simulations}

WARP has successfully been installed on $T E V$ and the first few runs have been completed. Nonetheless, the simulations are far from complete and many things need to be improved. This section outlines some of the steps that need to be implemented in the current code:

- First of all the code needs to be optimized in order to use less computational resources.

- Additional diagnostics and plotting tools should be implemented. For example it would be interesting to implement a few diagnostic windows that allow us to compare the transverse profiles from the simulations with those from the measurements in Tevatron Electron Lens Test Stand (TELTS).

- As discussed previously, the profile injection needs to be optimized in order to reduce the number of particles that are injected. Additionally to test whether the perveance and the beam current resemble measured values needs to be performed.

- The dumping of the simulations needs to be implemented, so that runs, which sometimes take a whole day, do not need to be repeated. 
- Extraction of simulation data is possible through the package ENV and should be implemented.

Furthermore, the TEL2 lattice or an optimal electron lens lattice for the Large Hadron Collider $(L H C)$ should be implemented. Using this deductions can be made on the effect of the electron lens bends on the Hollow Electron Beam (HEB). Furthermore it is possible to implement multiple species and sources in WARP, allowing the implementation of the accelerator proton beam and thus the direct simulation in WARP of the effect of the $H E B$ on the proton beam in one single pass. 


\section{Chapter 8}

\section{Conclusion}

The studies discussed in this thesis were completed in the context of supporting a conceptual design report on a Hollow Electron Beam Lens (HEBL) for proton beams at the Large Hadron Collider (LHC) at European Organization for Nuclear Research (CERN). Experiments conducted at the Tevatron over recent years have provided a solid basis for this beam scraping and halo control technique. Nonetheless, several further issues need to be addressed in the extrapolation of the $H E B L$ from the Tevatron to the $L H C$. In this thesis, we have discussed measurements and considerations that pertain to the design and feasibility of Hollow Electron Gun (HEG) hardware parameters, and to the effects of the Hollow Electron Beam (HEB) on the dynamics of the circulating proton beam.

For halo scraping, the inner radius of the $H E B$ is envisioned to be placed between $\approx 3 \sigma$ and $6 \sigma$ of the $L H C$ proton beam size. For the purpose of this thesis, $\sigma$ was set to $4.7 \times 10^{-4} \mathrm{~m}$. For stability reasons, the field in the guiding solenoids should be as large as possible. Considering previous experience and technical feasibilities, we considered a magnetic configuration of $\mathrm{B}=0.43-5-0.43 \mathrm{~T}\left(B_{\text {gun }}-B_{\text {main }}-B_{\text {coll }}\right)$. This implies a magnetic compression of a factor 3.4, which sets the cathode inner radius to $4 \sigma$ and the outer radius to $\approx 7.5 \sigma$.

A 1 inch Hollow Electron Gun (HG1b) based on a tungsten dispenser cathode was developed and tested. The perveance of this gun was determined to be $5.3(1) \times 10^{-6}$ perv, which is within $15 \%$ of numerical SAM simulations. This perveance implies a peak yield of over $5 \mathrm{~A}$ at $10 \mathrm{kV}$. Similar beam currents as were produced by the 0.6 inch Hollow Electron Gun (HG06) of 1.2 A, were measured using the $H G 1 b$ at cathode voltages of 3-4 kV. Based upon Tevatron experiments and numerical simulations for the $L H C$, this yield should be sufficient to smoothly scrape $7-\mathrm{TeV}$ protons.

During HEG characterization studies at the Tevatron Electron Lens Test Stand (TELTS), it was found that this larger $H G 1 b$ required a compression in order to obtain a transmission of $100 \%$ in the beam tube. Previous cathodes did not require such compressions. The best achievable configuration in the TELTS was found to be $B_{\text {gun }}=B_{\text {coll }}=0.25 B_{\text {main }}$. This configuration results in a 2 -fold compression of the $H E B$. Because the actual $H E B L$ will have a larger inner aperture, we do not expect transmission degradation to be an issue. By examining the dependence of the cathode yield on filament current, it was found that the optimal filament current is about $8.5 \mathrm{~A}$.

The transverse $H E B$ profiles were measured to estimate the symmetry of the resulting electric fields and possible adverse effects on the proton beam core. Extensive profile measurements as a function of beam current and solenoidal magnetic field were completed. These allowed us to study the evolution of the magnetically confined $H E B$ due to space charge. The profiles showed a scaling proportional to the accelerating voltage $V_{a}$ and inversely proportional to the square of the axial magnetic field $B$, which is in qualitative agreement with the theory of the diocotron instability. Consequently, profiles measured at very low cathode voltages and at the highest possible magnetic fields in the conventional test stand solenoids will be similar to the profiles in the actual $H E B L$ for the $L H C$ using the parameters suggested above.

Effects on the beam dynamics were studied by calculating the transverse electric fields generated by the measured profiles, and by estimating the resulting emittance growth. For typical operational 
parameters, the effect was much smaller that the natural emittance growth in the LHC. At higher $H E B$ currents or very low magnetic fields, the $H E B$ loses its azimuthal symmetry and electric fields in the center can no longer be considered negligible. This, together with the Tevatron experiments, provides a strong indication that it is possible to operate the $H E B L$ without negatively affecting the $L H C$ proton core. Nonetheless, this aspect will have to be further investigated by implementing the fields generated by the $H E B$ in a numerical tracking code.

In view of a realistic representation of the $H E B L$ in tracking simulations, the first full $3 \mathrm{D}$ calculation of the magnetized electron beam dynamics was completed using the WARP Particle-In-Cell (PIC) code. The $H E G$ was implemented in WARP together with the geometry of the TELTS. Three main effects could be verified: the magnetic compression of the beam, the evolution of the hollow profile, and some longitudinal micro-bunching towards the end of the drift tube.

This thesis work advanced the understanding of the physics and technology of Hollow Electron Beam Collimation $(H E B C)$ and provided the basis for further studies on the implementation of this technique at CERN. 


\section{Works Cited}

[1] X. Zhang, V. Shiltsev, A. Valishev, G. Stancari, G. Kuznetsov, G. Saewert, and V. Kamerdzhiev, "Operations of the Tevatron Electron Lenses," arXiv preprint arXiv:1207.6413, 2012.

[2] S. Redaelli US-LARP - CM20 - LHC Collimation Project Status, April 2013.

[3] V. Previtali, "Numerical simulations of a proposed hollow electron beam collimator for the LHC upgrade at CERN," Technical Memo FERMILAB-TM-2560-APC, Fermilab, July 2013.

[4] E. Rutherford, "Stability of atoms," Royal Society of London, pp. 389-394, June 1931.

[5] J. Cockcroft and E. Walton, "Experiments with high velocity positive ions," Proceedings of the Royal Society of London. Series A, vol. 129, no. 811, pp. 477-489, 1930.

[6] A. M. Sessler and E. J. Wilson, Engines of discovery: A Century of particle accelerators, vol. 17. World Scientific Hackensack, NJ, 2007.

[7] O. S. Brüning, P. Collier, P. Lebrun, S. Myers, R. Ostojic, J. Poole, and P. Proudlock, "The LHC Design Report, Vol. 1," CERN, Geneva, 2004.

[8] G. Stancari, "New methods of particle collimation in colliders," No. FERMILAB-CONF-11-506-AD-APC, (Providence, Rhode Island), American Physical Society, Brown University, 102011.

[9] N. Mokhov, J. Annala, R. Carrigan, M. Church, A. Drozhdin, T. Johnson, R. Reilly, V. Shiltsev, G. Stancari, D. Still, et al., "Tevatron beam halo collimation system: design, operational experience and new methods," Journal of Instrumentation, vol. 6, no. 08, p. T08005, 2011.

[10] A. Chao and M. Tigner, Handbook of accelerator physics and engineering. World scientific, 1999.

[11] G. Stancari, A. Valishev, G. Annala, G. Kuznetsov, V. Shiltsev, D. Still, and L. Vorobiev, "Collimation with hollow electron beams," Physical Review Letters, vol. 107, no. 8, p. 084802, 2011.

[12] J. Jeanneret, "Optics of a two-stage collimation system," Physical Review Special Topics-Accelerators and Beams, vol. 1, no. 8, p. 081001, 1998.

[13] N. V. Mokhov, "Beam collimation at hadron colliders," in AIP Conference Proceedings, vol. 693, p. 14, 2003.

[14] J. Jeanneret, D. Leroy, L. Oberli, and R. Trenkler, "Quench Levels and Transient Beam Losses in LHC Magnets," LHC Project Report 44, European Organization For Nuclear Research, LHC Division, CERN, CH-1211 Geneva 23, Switzerland, July 1996.

[15] N. V. Mokhov, A. I. Drozhdin, and G. W. Foster, "Beam-induced energy deposition issues in the Very Large Hadron Collider," in Particle Accelerator Conference, 2001. PAC 2001. Proceedings of the 2001, vol. 4, pp. 3171-3173, IEEE, 2001. 
[16] V. Shiltsev, "Tevatron electron lenses: Design and operation," Physical Review Special Topics Accelerators and Beams, vol. 11, no. 10, 2008.

[17] V. Shiltsev, "Considerations on compensation of beam-beam effects in the Tevatron with electron beams," Physical Review Special Topics - Accelerators and Beams, vol. 2, no. 7, 1999.

[18] X.-l. Zhang, K. Bishofberger, V. Kamerdzhiev, V. Lebedev, V. Shiltsev, R. Thurman-Keup, and A. Tollestrup, "Generation and diagnostics of uncaptured beam in the fermilab tevatron and its control by electron lenses," Physical Review Special Topics-Accelerators and Beams, vol. 11, no. 5, p. 051002, 2008.

[19] G. Stancari, "Beam-Beam Compensation Studies in the Tevatron with Electron Lenses," February 2013.

[20] W. Fischer, Z. Altinbas, M. Anerella, M. Blaskiewicz, D. Bruno, W. Dawson, D. Gassner, X. Gu, R. Gupta, K. Hamdi, et al., "Commissioning progress of the RHIC electron lenses," tech. rep., Brookhaven National Laboratory (BNL) Relativistic Heavy Ion Collider, 2013.

[21] V. Previtali, G. Stancari, A. Valishev, and S. Redaelli, "Numerical simulations of a hollow electron lens as a scraping device for the LHC," MOPWO044, IPAC13.

[22] G. Stancari, G. Annala, T. Johnson, D. Still, and A. Valishev, "Measurements of transverse beam diffusion rates in the Fermilab Tevatron collider," PAC11, San Sebastian, Spain, p. 1882, 2011.

[23] G. Stancari, G. Annala, V. Shiltsev, D. Still, A. Valishev, and L. Vorobiev, "Experimental study of magnetically confined hollow electron beams in the Tevatron as collimators for intense high-energy hadron beams," in Conf. Proc. C, vol. 110328, p. 370, 2011.

[24] C. Crawford, A. Sery, V. Shiltsev, A. Aleksandrov, B. Skarbo, and B. Sukhina, "Magnetic field alignment in the beam-beam compensation device," in Particle Accelerator Conference, 1999. Proceedings of the 1999, vol. 5, pp. 3321-3323, IEEE, 1999.

[25] M. Reiser, Theory and design of charged particle beams. Weinheim: Wiley-VCH, 2nd, updated and expanded ed. ed., 2008.

[26] G. Stancari, A. Drozhdin, G. Kuznetsov, V. Shiltsev, D. Still, A. Valishev, L. Vorobiev, R. Assmann, and A. Kabantsev, "Development of hollow electron beams for proton and ion collimation," in Conf. Proc. C100523: tupeb076, 2010, no. FERMILAB-CONF-10-182-AD-APC, Fermi National Accelerator Laboratory (FNAL), Batavia, IL, 2010.

[27] A. Gilmour, Principles of Klystrons, Traveling Wave Tubes, Magnetrons, Cross-Field Amplifiers, and Gyrotrons. Artech House microwave library, Artech House, Incorporated, 2011.

[28] S. Li, "Characterization of an Electron Gun for Hollow Electron Beam Collimation," Tech. Rep. FERMILAB-TM-2542-APC, Fermilab, Fermi National Accelerator Laboratory, P.O. Box 500, Batavia, Illinois 60510, USA, August 2012.

[29] A. Ivanov and M. Tiunov, "ULTRASAM-2D code for simulation of electron guns with ultra high precision," Proceeding of EPAC-2002, Paris, pp. 1634-1636, 2002.

[30] L. Vorobiev, "Private communications." Fermilab, 2013.

[31] W. Umrath et al., "Fundamentals of vacuum technology," Leybold, Cologne, 1998.

[32] G. Stancari, "Private communications." Fermilab, 2013.

[33] R. C. Davidson, Physics of nonneutral plasmas, vol. 5. Addison-Wesley New York, 1990. 
[34] E. Buckingham, "On physically similar systems; illustrations of the use of dimensional equations," Physical Review, vol. 4, no. 4, pp. 345-376, 1914.

[35] D. P. Grote et al., "WARP Manual," Lawrence Livermore National Laboratory, vol. 7000, pp. 94550-9234, April 2000.

[36] M. Conte and W. W. MacKay, An introduction to the physics of particle accelerators, vol. 160. World Scientific, 1991.

[37] J. Rossbach and P. Schmüser, "Basic course on accelerator optics," CERN European Organization for Nuclear Research-Reports-CERN, pp. 17-17, 1994.

[38] D. Möhl, "Sources of emittance growth," Proceedings of the CAS. Specialised CAS Course on Small Accelerators, Zeegse, The Netherlands, May 24-June 2, pp. 2006-012, 2005.

[39] G. Valentino, R. Aßmann, R. Bruce, F. Burkart, V. Previtali, S. Redaelli, B. Salvachua, G. Stancari, and A. Valishev, "Beam diffusion measurements using collimator scans in the LHC," Physical Review Special Topics-Accelerators and Beams, vol. 16, no. 2, p. 021003, 2013.

[40] R. W. Hockney and J. W. Eastwood, Computer simulation using particles. CRC Press, 2010. 
Appendices 


\section{Appendix A}

\section{LHC and Tevatron Parameters}

\begin{tabular}{|llr|r|r|}
\hline \hline Quantity & Symbol & Unit & Tevatron $[16,9]$ & LHC [7] \\
\hline \hline Collider type & & $p-\bar{p}$ & $p-p$ \\
Circumference & $C$ & {$[\mathrm{~m}]$} & $6.28 \mathrm{~km}$ & $26.66 \mathrm{~km}$ \\
\# Beam Pipes & & 1 & 2 \\
\# of IRs & $N_{R}$ & & 2 & 8 \\
Injection Energy & $E_{\text {inj }}$ & {$[\mathrm{GeV}]$} & $120 / 150$ & 450 \\
Nominal Energy & $E_{n o m}$ & {$[\mathrm{TeV}]$} & 0.98 & 7 \\
Achieved Energy & $E$ & {$[\mathrm{TeV}]$} & 0.980 & 4.5 \\
\# of bunches & $N_{b}$ & & $36(3$ bunch trains $)$ & 2808 \\
Protons per bunch & $N_{p}$ & & $2.7 \times 10^{11} / 1.35 \times 10^{11}$ & $1.15 \times 10^{11}$ \\
Bunch spacing & $T_{b}$ & {$[\mathrm{~ns}]$} & 396 & 25 \\
Nominal Beam Intensity & $N_{p}, N_{\bar{p}}$ & & $9.72 \times 10^{12}, 4.86 \times 10^{12}$ & $3.23 \times 10^{14}$ \\
Peak Luminosity & $L_{0}$ & {$\left[\mathrm{~cm}^{-2} \mathrm{~s}^{-1}\right]$} & $3.15 \times 10^{32}$ & $2.3 \times 10^{34}$ \\
Nominal Emittance $(\mathrm{RMS})$ & $\varepsilon$ & {$[\mu \mathrm{m}]$} & $2.8 / 1.4$ & 3.75 \\
Nominal Energy Density & $U$ & {$\left[\mathrm{MJ} / \mathrm{mm}^{2}\right]$} & 2 & 360 \\
Cryogenic Temperature & $T$ & {$[\mathrm{~K}]$} & 4 & 1.9 \\
\hline \hline
\end{tabular}

Table A.1: Important parameters of Tevatron and LHC

It is important to notice that the proton and anti-proton beams in the Tevatron share the same beam pipe, in which they are transversely displaced. Beam-Beam effects are thus significantly more important at the Tevatron. In the LHC, both beams have their own channels, which intersect at 4 Interaction Regions (IRs), where the experiments ATLAS, CMS, ALICE, LHCb and others can be found. 


\section{Appendix B}

\section{Measured Profiles}

\section{B.1 List of measured profiles}

Table B.1: Table showing all profile measurements made.

\begin{tabular}{|c|c|c|c|c|c|c|c|c|c|}
\hline$\#$ & Date & $\begin{array}{l}\text { Magnetic Field } \\
{[\mathrm{T}]}\end{array}$ & $\begin{array}{l}\text { Cathode } \\
\text { Poten- } \\
\text { tial } \\
{[\mathrm{kV}]}\end{array}$ & $\begin{array}{l}\text { Cathode } \\
\text { Current } \\
{[\mathrm{mA}]}\end{array}$ & $\begin{array}{l}\text { Avg. } \\
\text { Current } \\
{[\mathrm{mA}]}\end{array}$ & $\begin{array}{l}\text { Collector } \\
\text { Current } \\
{[\mathrm{mA}]}\end{array}$ & $\begin{array}{l}\text { Rep. } \\
\text { Rate } \\
{[\mathrm{Hz}]}\end{array}$ & $\begin{array}{l}\text { Vacuum } \\
{[\mathrm{V}]}\end{array}$ & $\begin{array}{l}\text { Pulse } \\
\text { Length } \\
{[m u s]}\end{array}$ \\
\hline 1 & 20.02 .1311 .35 & $0.1-0.1-0.1$ & 0.9 & $\mathrm{~N} / \mathrm{A}$ & $\mathrm{N} / \mathrm{A}$ & 118 & 500 & $\mathrm{~N} / \mathrm{A}$ & 8 \\
\hline 2 & 21.02 .1311 .00 & $0.2-0.2-0.2$ & 3.6 & $\mathrm{~N} / \mathrm{A}$ & $\mathrm{N} / \mathrm{A}$ & 928 & 50 & $\mathrm{~N} / \mathrm{A}$ & 8 \\
\hline 3 & 21.02 .1311 .40 & $0.1-0.1-0.1$ & 2.0 & $\mathrm{~N} / \mathrm{A}$ & $\mathrm{N} / \mathrm{A}$ & 398 & 200 & $\mathrm{~N} / \mathrm{A}$ & 8 \\
\hline 4 & 21.02 .1313 .05 & $0.1-0.1-0.1$ & 3.0 & $\mathrm{~N} / \mathrm{A}$ & $\mathrm{N} / \mathrm{A}$ & 726 & 100 & $\mathrm{~N} / \mathrm{A}$ & 8 \\
\hline 5 & 04.04 .1309 .00 & $0.25-0.25-0.25$ & 5.6 & 2322 & 1.21 & 1770 & 20 & 6.48 & 8 \\
\hline 6 & 04.04 .13 & $0.25-0.25-0.25$ & 5.0 & 1980 & 1.25 & 1484 & 30 & 6.41 & 8 \\
\hline 7 & 04.04 .1312 .25 & $0.25-0.25-0.25$ & 2.1 & 548 & 1.33 & 412 & 200 & 6.47 & 8 \\
\hline 8 & 04.04 .1313 .45 & $0.25-0.25-0.25$ & 3.5 & 1156 & 1.35 & 865 & 80 & 6.41 & 8 \\
\hline 9 & 04.04 .1315 .08 & $0.3-0.3-0.3$ & 5.0 & 1964 & 1.24 & 1460 & 30 & 6.41 & 8 \\
\hline 10 & 05.04 .1308 .30 & $0.2-0.2-0.2$ & 1.3 & 282 & 1.33 & 212 & 400 & 6.57 & 8 \\
\hline 11 & 05.04 .1310 .00 & $0.2-0.2-0.2$ & 2.2 & 592 & 1.335 & 448 & 180 & 6.57 & 8 \\
\hline 12 & 05.04 .1311 .20 & $0.2-0.2-0.2$ & 3.1 & 972.6 & 1.35 & 744.4 & 100 & 6.52 & 8 \\
\hline 13 & 05.04 .1312 .45 & $0.15-0.15-0.15$ & 0.75 & 120.8 & 1.26 & 90.4 & 800 & 6.52 & 8 \\
\hline 14 & 05.04 .1314 .15 & $0.15-0.15-0.15$ & 2.0 & 506 & 1.25 & 390 & 200 & 6.51 & 8 \\
\hline 15 & 05.04 .1315 .47 & $0.15-0.15-0.15$ & 1.3 & 254 & 1.21 & 192 & 400 & 6.52 & 8 \\
\hline 16 & 02.05 .13 & $0.25-0.25-0.25$ & 0.50 & 69.6 & 0.96 & 51 & 1000 & 6.64 & 8 \\
\hline 17 & 02.05 .13 & $0.2-0.2-0.2$ & 0.50 & 68.4 & 0.94 & 49 & 1000 & 6.60 & 8 \\
\hline 18 & 02.05 .13 & $0.3-0.3-0.3$ & 0.50 & 68.4 & 0.94 & 56 & 1000 & 6.60 & 8 \\
\hline 19 & 07.05 .13 & $0.3-0.3-0.3$ & 0.50 & 70.8 & 0.98 & 50.4 & 7000 & 6.58 & 8 \\
\hline 20 & 13.05 .13 & $0.3-0.3-0.3$ & 0.50 & 74.4 & 1.05 & 50.2 & 1000 & 6.64 & 8 \\
\hline 21 & 13.05 .13 & $0.3-0.3-0.3$ & 0.50 & $\mathrm{~N} / \mathrm{A}$ & 0.36 & $\mathrm{~N} / \mathrm{A}$ & 300 & 6.64 & 8 \\
\hline 22 & 13.05 .13 & $0.3-0.3-0.3$ & 0.50 & 70.4 & 0.99 & 50.8 & 1000 & 6.60 & 8 \\
\hline 23 & 13.05 .13 & $0.3-0.3-0.3$ & 0.50 & $\mathrm{~N} / \mathrm{A}$ & 0.34 & $\mathrm{~N} / \mathrm{A}$ & 300 & 6.60 & 8 \\
\hline 24 & 13.05 .13 & $0.1-0.3-0.1$ & 0.50 & 72.4 & 1.02 & 69.8 & 1000 & $\mathrm{~N} / \mathrm{A}$ & 8 \\
\hline 25 & 14.05 .1320 .21 & $0.08-0.32-0.08$ & 0.50 & 74.4 & 1.02 & 72.8 & 1000 & $\mathrm{~N} / \mathrm{A}$ & 8 \\
\hline 26 & 14.05 .1320 .22 & $0.08-0.32-0.08$ & 1.0 & 198 & 0.98 & 195 & 400 & $\mathrm{~N} / \mathrm{A}$ & 8 \\
\hline 27 & 14.05 .1320 .24 & $0.08-0.32-0.08$ & 2.0 & 528 & 1.07 & 526 & 150 & $\mathrm{~N} / \mathrm{A}$ & 8 \\
\hline 28 & 14.05 .1320 .31 & $0.08-0.32-0.08$ & 4.0 & 1460 & 1.13 & 1450 & 40 & $\mathrm{~N} / \mathrm{A}$ & 8 \\
\hline 29 & 15.05 .1311 .49 & $0.08-0.32-0.08$ & 8.0 & 3760 & 1.57 & 3700 & 10 & $\mathrm{~N} / \mathrm{A}$ & 8 \\
\hline 30 & 15.05 .1312 .31 & $0.08-0.32-0.08$ & 7.0 & 3160 & 1.33 & 3100 & 10 & $\mathrm{~N} / \mathrm{A}$ & 8 \\
\hline 31 & 15.05 .1313 .22 & $0.08-0.32-0.08$ & 0.25 & 27.0 & 1.09 & 25.8 & 2500 & $\mathrm{~N} / \mathrm{A}$ & 8 \\
\hline 32 & 15.05 .13 & $0.08-0.32-0.08$ & 0.125 & 9.72 & 0.91 & 9.32 & 4000 & $\mathrm{~N} / \mathrm{A}$ & 8 \\
\hline 33 & 15.05 .13 & $0.08-0.32-0.08$ & 3.0 & 956 & 1.32 & 944 & 100 & $\mathrm{~N} / \mathrm{A}$ & 8 \\
\hline 34 & 15.05 .1315 .39 & $0.08-0.32-0.08$ & 5.0 & 7990 & 1.01 & 7990 & 15 & $\mathrm{~N} / \mathrm{A}$ & 8 \\
\hline 35 & 15.05 .13 & $0.08-0.32-0.08$ & 6.0 & 2560 & 1.14 & 2530 & 10 & $\mathrm{~N} / \mathrm{A}$ & 8 \\
\hline 36 & 15.05 .1317 .53 & $0.1-0.4-0.1$ & 1.0 & 197 & 0.97 & 196 & 400 & $\mathrm{~N} / \mathrm{A}$ & 8 \\
\hline 37 & 15.05 .1318 .51 & $0.1-0.4-0.1$ & 3.125 & 1024 & 1.4 & 1022 & 100 & $\mathrm{~N} / \mathrm{A}$ & 8 \\
\hline 38 & $15.05 .1319: 20$ & $0.1-0.4-0.1$ & 6.250 & 2780 & 1.16 & 2754 & 10 & $\mathrm{~N} / \mathrm{A}$ & 8 \\
\hline 39 & $16.05 .1310: 45$ & $0.06-0.24-0.06$ & 1.0 & 798 & 0.99 & 796 & 400 & $\mathrm{~N} / \mathrm{A}$ & 8 \\
\hline 40 & $16.05 .1311: 02$ & $0.04-0.16-0.04$ & 1.0 & 797 & 0.99 & 795 & 400 & $\mathrm{~N} / \mathrm{A}$ & 8 \\
\hline 41 & $16.05 .1311: 46$ & $0.04-0.16-0.04$ & 0.50 & 74.4 & 1.02 & 72.4 & 7000 & $\mathrm{~N} / \mathrm{A}$ & 8 \\
\hline 42 & $20.05 .1311: 40$ & $0.1-0.4-0.1$ & 0.50 & 73.6 & 1.02 & 72.8 & 1000 & $\mathrm{~N} / \mathrm{A}$ & 8 \\
\hline
\end{tabular}




\begin{tabular}{|l|l|l|l|l|l|l|l|l|l|}
\hline 43 & $20.05 .1311: 42$ & $0.1-0.4-0.1$ & 2.0 & 530 & 1.06 & 534 & 750 & N/A & 8 \\
\hline 44 & $20.05 .1312: 19$ & $0.1-0.4-0.1$ & 4.0 & 1463 & 1.13 & 1440 & 40 & N/A & 8 \\
\hline 45 & $20.05 .1312: 59$ & $0.1-0.4-0.1$ & 5.0 & 1990 & 1.01 & 1980 & 15 & N/A & 8 \\
\hline 46 & $20.05 .1313: 40$ & $0.1-0.4-0.1$ & 8.0 & 3880 & 1.56 & 3880 & 10 & N/A & 8 \\
\hline 47 & $20.05 .1314: 33$ & $0.04-0.16-0.04$ & 2.0 & 498 & 1.03 & 488 & 150 & N/A & 8 \\
\hline 48 & $20.05 .1315: 21$ & $0.04-0.16-0.04$ & 0.75 & 127.2 & 0.83 & 125.5 & 500 & N/A & 8 \\
\hline 49 & $21.05 .1310: 57$ & $0.04-0.16-0.04$ & 1.5 & 338.5 & 0.92 & 332.5 & 200 & N/A & 8 \\
\hline 50 & $21.05 .1314: 10$ & $0.04-0.16-0.04$ & 2.5 & 682 & 1.04 & 658 & 100 & N/A & 8 \\
\hline 51 & $21.05 .1314: 50$ & $0.06-0.24-0.06$ & 0.50 & 74.0 & 0.98 & 73.2 & 7000 & N/A & 8 \\
\hline 52 & 21.05 .13 & $0.06-0.24-0.06$ & 2.0 & 528 & 1.07 & 526 & 150 & N/A & 8 \\
\hline 53 & $21.05 .1315: 31$ & $0.06-0.24-0.06$ & 3.0 & 928 & 1.32 & 923.8 & 100 & N/A & 8 \\
\hline 54 & $21.05 .1316: 18$ & $0.06-0.24-0.06$ & 4.0 & 1384 & 1.11 & 1368 & 40 & N/A & 8 \\
\hline
\end{tabular}




\section{Appendix C}

\section{Codes}

\section{C.1 Profile Measurement}

\section{C.1.1 Data Acquisition - ACL script}

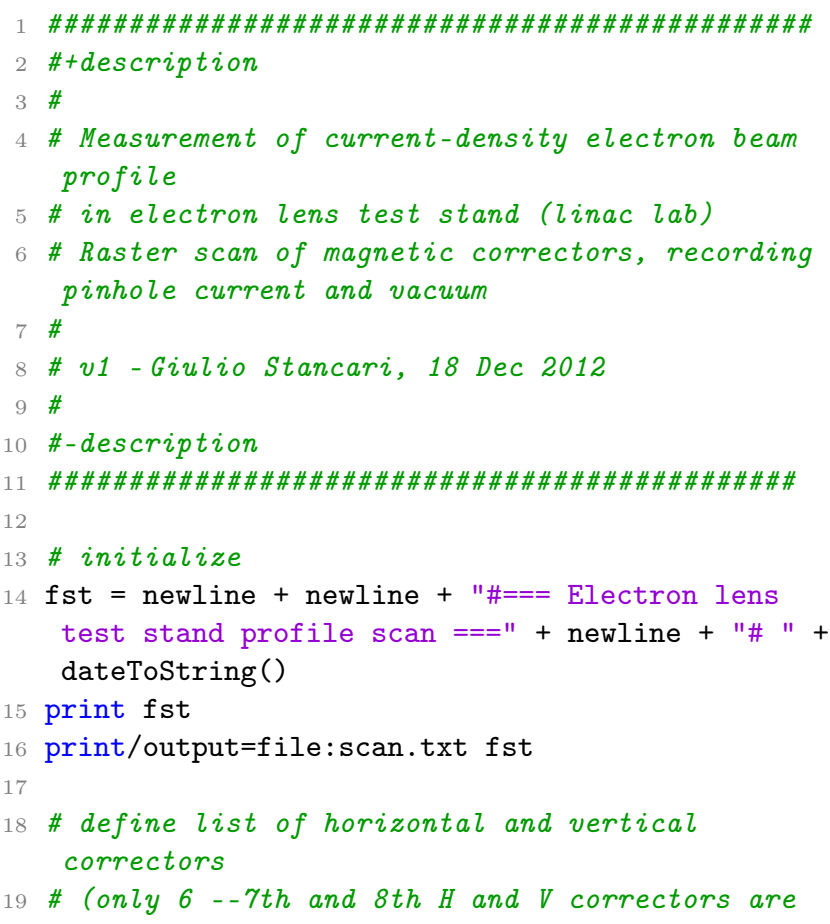

20 deviceList/create hcor devices=' Z:HCORO2, Z:HCOR06 , Z:HCORB2, Z:HCOR10, Z :HCOR14, Z:HCORB6'

21 deviceList/create vcor devices='Z:VCOR01,Z:VCOR05 , Z:VCORB1, Z:VCOR09, Z:VCOR13, Z :VCORB5,

\# define list of variables to read

\# $Z: B C 1 A D 3=$ probe current

\# Z:BC1AD5 = tube current

\# Z:BC2AD5 = diode pump vacuum

27 devicelist/create devin devices=' $\mathrm{Z}: \mathrm{BC} 1 \mathrm{AD} 3, \mathrm{Z}$ : BC1AD5, Z : BC2AD5, Z : HCOR02, Z : HCOR06, Z : HCORB2, Z : HCOR10, Z: HCOR14, Z: HCORB6, Z : VCOR01, Z : VCOR05, Z : VCORB1, Z:VCOR09, Z:VCOR13, Z:VCORB5,

8 devicelist/size/output=variable:ndevin devin 9 declare rd float [15]
31 \# define scan parameters

$2 \# \operatorname{mix}=$ minimum value of horizontal correctors [A]

$\# \max =\operatorname{maximum} .$. .

\# miy = minimum value of vertical correctors [A]

\# may = maximum. .

\# step = step size [A]

\#dt = time spent at each point [s]

38 input/user/float/default=-1 inmix "Min. H value ( $\operatorname{mix})[\mathrm{A}] "$

$39 \operatorname{mix}=\langle$ inmix $>$

40 input/user/float/default=1 inmax "Max. H value ( $\max )[\mathrm{A}] "$

$41 \max =\langle$ inmax $>$

42 input/user/float/default=-1 inmiy "Min. V value ( miy) $[\mathrm{A}] "$

43 miy $=\langle$ inmiy $>$

44 input/user/float/default=1 inmay "Max. V value ( may) $[\mathrm{A}] "$

45 may $=\langle$ inmay $>$

46 input/user/float/default=0.5 instep "Step size [A ]"

47 step $=$ <instep>

48 input/user/float/default=1 indt "Time interval [s ]"

$49 \mathrm{dt}=\langle$ indt $\rangle$

fst = "\# Scan parameters:" + newline + "\# $\operatorname{mix}=$ "

+ toString $(\operatorname{mix}, 8)+" \mathrm{~A}^{\prime}+$ newline $+" \# \max ="$

+ toString $(\max , 8)+" \mathrm{~A} "+$ newline + "\# miy = "

+ toString(miy, 8) + "A" + newline + 1

"\# may = " + toString (may, 8) + "A"+newline+"

\# step = "+toString (step,8)+" A"+newline+"\# dt

$=$ "+toString $(\mathrm{dt}, 8)+" \mathrm{~s} "$

print fst

print/output=file: scan.txt fst

\# validating scan parameters

if $(\operatorname{mix}<-5)$ or $(\max >5)$ or (miy $<-5$ ) or (may $>5$ ) or 1 $(\operatorname{mix}>\max )$ or $(\operatorname{miy}>\operatorname{may})$ or $\backslash$

(step <=0) or (step > 5) or 1

$(\mathrm{dt}<=0)$ or $(\mathrm{dt}>600)$ 

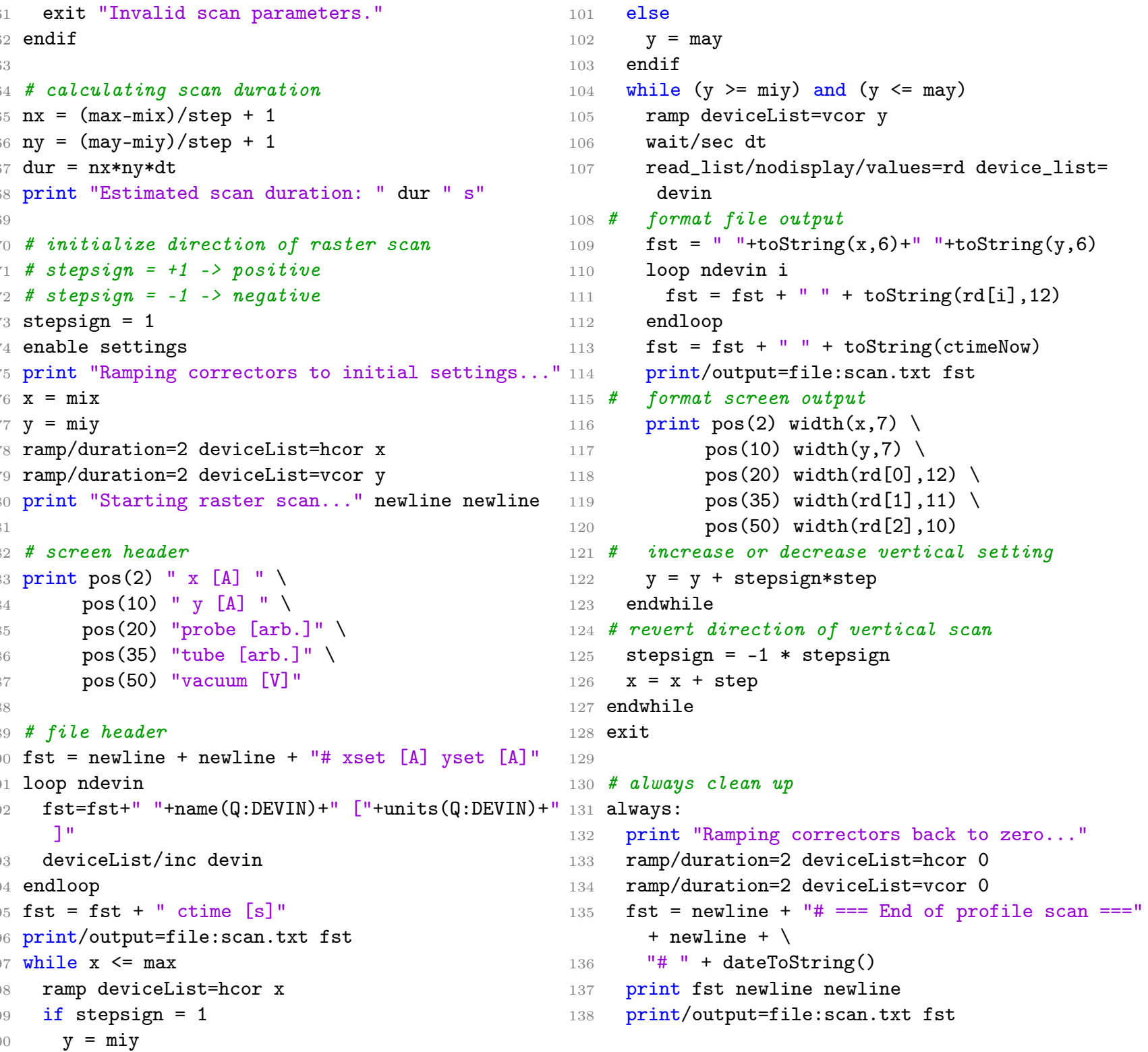

\section{C.1.2 Data Processing - R script}

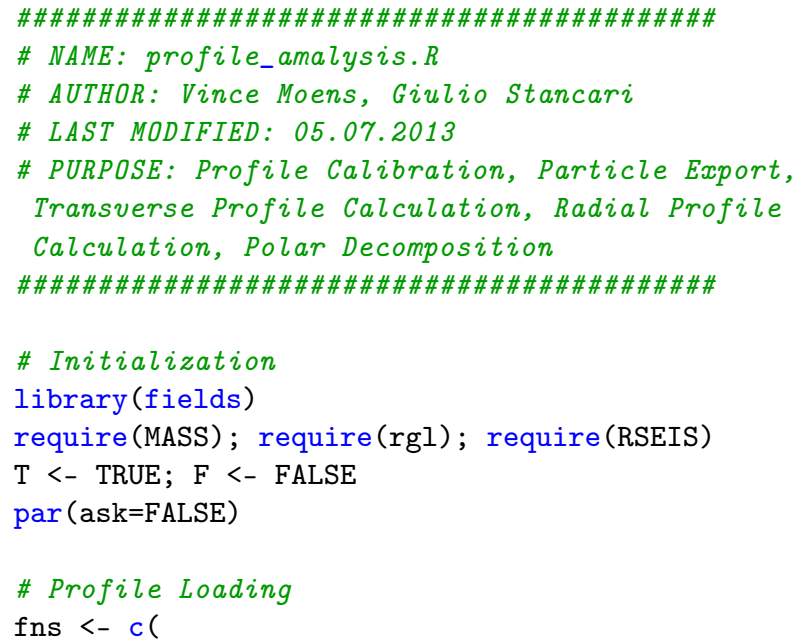

". ./Data/HG/HG_091023_775A_250V_303030kG_16mA. dat", \#1

". ./Data/HG1/HG1b_130521_9p25A_06-24-06kG_4kV_ 1368mA.txt.gz" \#136

\#\# CONTROL SWITCHES \#\#\#

profile.list <- 99:100 \#length(fns)

CORRECT . SCALLOPS <- T

FIND. SCALLOP. CORRECTION. PAR <- F

SUBTRACT.BACKGROUND <- T

CORRECT . BOTTOM. LEFT <- T

NORMALIZE. PROFILE $<-$ T

CALIBRATE. CORRECTORS <- F 


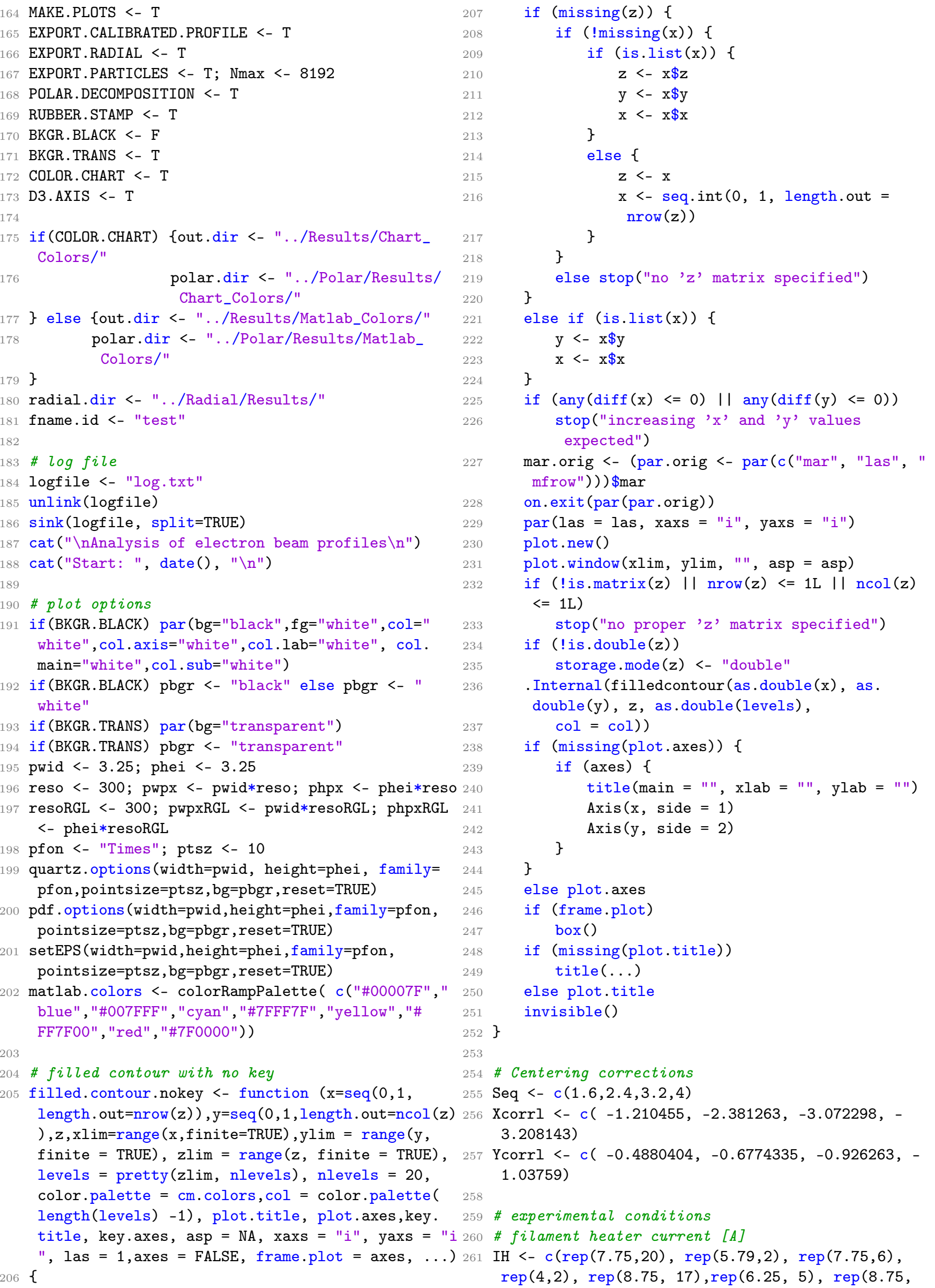


2), $\operatorname{rep}(9.25,10), \operatorname{rep}(7.25,2), \operatorname{rep}(9.25,30)$, $\operatorname{rep}(10.0,4), \operatorname{rep}(9.25,36))$

262 \# cathode voltage [V]

$263 \mathrm{~V}<-c(250,500,1000,1500,2000,3000,4000$, $5000,6000,7500,9000,3 e 3,3 e 3,9 e 3,9 e 3,9 e 3$, $250,250,1 \mathrm{e} 3,6 \mathrm{e} 3,500,7500,500,2000,7500$, $7500,50,2000,100,5000,500,1 \mathrm{e}, 2 \mathrm{e} 3,4 \mathrm{e}, 8$ e3, $1 \mathrm{e} 3,2 \mathrm{e} 3,500,4 \mathrm{e} 3,8 \mathrm{e} 3,500,500,1 \mathrm{e} 3,2 \mathrm{e} 3$, $4 \mathrm{e} 3,8 \mathrm{e}, 3 \mathrm{e} 3,8 \mathrm{e} 3,2 \mathrm{e} 3,500,500,500,8 \mathrm{e}, 500$, $1 \mathrm{e} 3,5 \mathrm{e} 3,500,8 \mathrm{e}, 4 \mathrm{e} 3,2 \mathrm{e}, 1 \mathrm{e} 3,6 \mathrm{e}, 3 \mathrm{e} 3,7 \mathrm{e} 3$ , rep $(8 \mathrm{e} 3,2), 889,2 e 3,3 e 3,3556,5556,5000$, $2083,3472,5000,1333,2222,3111,750,2000$, 1250, $\operatorname{rep}(500,23), 1 \mathrm{e} 3,2 \mathrm{e} 3,4 \mathrm{e}, 8 \mathrm{e} 3,7 \mathrm{e} 3,250$

$125,3 e 3,5 e 3,6 e 3,1 e 3,3125,6250, \operatorname{rep}(1 e 3,5)$ , 500, 500, 2000, 4000, 5000, 8000, 2000, 750, $1500,2500,500,2000,3000,4000)$

264 \# peak beam current at collector [A]

265 I <- $c(0.016,0.044,0.121,0.220,0.325,0.582$, $0.876,1.180,1.490,2.020,2.490,0.564,0.600$, $2.330,2.470,2.550,0.016,0.015,0.118,1.46$, $0.023,0.074,0.044,0.330,2.040,1.984,1460 \mathrm{e}-$ $6,0.348,0.00198,1.670,0.050,0.134,0.386$, $1.030,2.850,0.134,0.394,0.049,1.064,2.860$, $0.050,0.050,0.135,0.376,1.056,2.940$, $0.692,0.140,0.098, \operatorname{rep}(0.048,3), 2.860,0.050$, $0.004,0.013,0.051,2.94,1.1, .388, .14$, $1.924, .694,2.394, .440, .027,0.118, .398$, $.726, .928,1.770,1.484, .412, .865,1.460$, $.213, .448,0.744, .090, .390, .192, \operatorname{rep}(.051,3)$ , $\operatorname{rep}(.049,5), \operatorname{rep}(.051,6), \operatorname{rep}(0.050,5), \operatorname{rep}$ $(0.070,2), \operatorname{rep}(0.073,2), 0.195,0.526,1.45$, $3.7,3.1,0.026,0.009,0.944,1.99,2.53$, $0.195,1.022,2.754,0.196, \operatorname{rep}(0.195,4), 0.072$, $0.073,0.0534,1.44,1.98,3.88,0.488,0.125$, $0.332,0.658,0.073,0.526,0.924,1.368)$

266 \# solenoidal field [T]

267 B <- $c(r e p(.3,11), 0.1,0.4,0.1,0.2,0.4,0.1$, $0.033,0.133,0.223, \operatorname{rep}(0.3,7), 0.4, \operatorname{rep}(0.4,2)$, $\operatorname{rep}(0.3,5), \operatorname{rep}(0.1,5), 0.4, \operatorname{rep}(0.2,5), 0.3$, $\operatorname{rep}(0.3,7), \operatorname{rep}(0.2,2), \operatorname{rep}(0.3,10), \operatorname{rep}(0.1,3)$ , $0.2, \operatorname{rep}(0.25,4), 0.3, \operatorname{rep}(0.2,3), \operatorname{rep}(0.15,3)$ , $\operatorname{rep}(0.25,3), \operatorname{rep}(0.2,5), \operatorname{rep}(0.3,13)$, rep $(0.32,12), \operatorname{rep}(0.4,3), 0.24, \operatorname{rep}(0.16,5)$, rep $(0.4,5), \operatorname{rep}(0.16,4), \operatorname{rep}(0.24,4))$

268 \# number of correctors

$269 \mathrm{NC}<-c(\operatorname{rep}(4,11), 3,6,3,3,6,3,3,3,3$, rep $(6,7), 4, \operatorname{rep}(4,2), \operatorname{rep}(6,106))$

270 \# raster-scan correction Parameters

271 besttp <- c $(-0.6069,-0.5819,-0.7744,-0.8363$, $0.5632,-0.5392,-0.3877,-1.017,-0.5627$, $0.5051,-0.7392,0.0392,-1.9296,-0.1264,-$ $0.3909,-1.631,-0.2347,-0.2685,-0.1528$, $0.0197,-1.7174,-0.7895,-1.2949,-1.5334$, $1.6833,-1.2,-1.19,-4.02,-0.37,-0.44,-$ $0.3901180,-0.4279687,-0.4502339,-0.3418769$, $0.2973467,-0.3418769,-0.3203540,-0.3233227$, $0.2928937,-0.3671107,-0.4932798,-0.4583977$, $0.4895689,-0.4784364,-0.2832455,-0.2832455,-$ $0.3878915,-0.350783, \operatorname{rep}(-0.4,6), \operatorname{rep}(-0.62$, 2), $\operatorname{rep}(-0.62,8),-.18,-.62,-0.3418769$, $0.3203540,-0.3, \operatorname{rep}(-0.2832455,67))$

272 \# calibration constants [mm*T/A/corr]

273 calx.v <- $\operatorname{rep}(0.13$, length(fns))
274 caly.v <- rep (0.14, length(fns))

275 calx.v[which $(\mathrm{NC}==3)]<-0.1285$ \# checked

276 calx.v[20]<- $\quad 0.1526 \# N C=3$ but different settings

calx.v[which $(\mathrm{NC}==4)]<-0.1313$ \# checked

calx.v[which $(\mathrm{NC}==6)]<-0.1706$ \# checked

calx.v[29:30]<- $0.199 \# 3.5-4.0-1.5 k G$, Gaussian gun

caly.v[which $(\mathrm{NC}==3)]<-0.1389$ \# checked

caly.v[20]<- $\quad 0.1436$ \#NC=3 but different settings

caly.v $[$ which $(\mathrm{NC}==4)]<-0.1450$ \# checked

caly.v $[$ which $(\mathrm{NC}==6)]<-0.1793$ \# checked

caly.v[29:30]<- 0.216 \#3.5-4.0-1.5 kG, Gaussian gun

\# offsets [A]

xoff.v <- $\operatorname{rep}(0$, length(fns))

yoff.v <- rep (0, length(fns))

xoff.v[which $(\mathrm{NC}==4)]<--0.4109$

xoff.v[which $(\mathrm{NC}==3)]<-\mathrm{c}(-0.0199,-0.0895$, $0.3217,-0.0787,-0.0081,-0.1633,0)$ \# $N C=3$

291 xoff.v[20] <- $0.5942 \# N C=3$ special case

292 xoff.v[which $(\mathrm{NC}==6)]<--0.3265 \# N C=6$

xoff.v $[$ which $(\mathrm{NC}==8)]<--0.1772 \# N C=8$

294 xoff.v[29:30]<- -1.2 \# 3.5-4.0-1.5 kG

295 xoff.v[c(31:35, 47:54,57:66)]<- $-0.419 \# 1$-in hollow gun, $3 \mathrm{kG}$

xoff.v[c(36:40,67:69)]<- -0.114\#1-in hollow gun, $1 \mathrm{kG}$

297 xoff.v[41] <- $-0.58 \# 1$-in hollow gun, $4 k G$, to be verified

xoff.v[c(42:46, 55:56,70)]<- -0.23\#1-in hollow gun, $2 k G$

299 yoff.v[which $(\mathrm{NC}==4)]<-\quad-0.1157$

300 yoff.v[which $(\mathrm{NC}==3)]<-c(-0.0533,-0.0872$, $0.0786,-0.0469,-0.0330,-0.0582,0) \# N C=3$ yoff.v[20]<- $0.1957 \# N C=3$ special case yoff.v $[$ which $(\mathrm{NC}==6)]<--0.1000 \# N C=6$ yoff. $\mathrm{v}[$ which $(\mathrm{NC}==8)]<--0.0510 \# N C=8$

yoff.v[29:30]<- -0.40\#3.5-4.0-1.5 kG

305 yoff.v[c $(31: 35,47: 54,57: 66)]<-0.081 \# 1$-in hollow gun, $3 \mathrm{kG}$

306 yoff.v[c(36:40,67:69)]<- 0.033 \#1-in hollow gun, $1 \mathrm{kG}$

yoff.v[41]<- 0.067 \#1-in hollow gun, $4 k G$, to be verified

308 yoff.v[c(42:46, 55:56,70)]<- $0.07 \# 1$-in hollow gun, $2 k G$

309

310 \# inner ( $r 1$ ) and outer (r2) gun radii [m]

$311 \mathrm{r} 1<-c(\operatorname{rep}(0.00450,28), \operatorname{rep}(0.00000,2)$, rep $(0.00675,106))$

312 r2 <- c $(\operatorname{rep}(0.00762,28), \operatorname{rep}(0.00508,2)$, rep $(0.01270,106))$

313 xylim.fudge <- 1.2 \# fudge factor for xy limits = r2* xylim.fudge

\section{4}

315 \# constants

316 c $<-299792458$

317 eps $0<-8.854187817 \mathrm{e}-12$

318 me $<-9.1093826 \mathrm{e}-31$

$319 \mathrm{q}<-1.60217653 e-19$ 
$320 \mathrm{~L}<-\operatorname{rep}(2.86$, length(fns)) \# anode-collector distance $[\mathrm{m}]$

321 \# electron velocities

22 gamma $<-1+\mathrm{q} * \mathrm{~V} /\left(\right.$ me*c $\left.\mathrm{c}^{-2}\right)$

23 beta <- sqrt (gamma*gamma-1)/gamma

$24 \mathrm{v}<-$ beta*c

5 \# flight times

$6 \mathrm{t}<-\mathrm{L} / \mathrm{v}$

\# average densities

lambda $<-I /(q * v)$ \# linear

$\mathrm{n}<-\mathrm{lambda} /(\mathrm{pi} *(\mathrm{r} 2 * \mathrm{r} 2-\mathrm{r} 1 * \mathrm{r} 1))$ \# volume

\# plasma frequencies

wp <- $\operatorname{sqrt}(\mathrm{n} * \mathrm{q} * \mathrm{q} /(\mathrm{eps} 0 * \mathrm{me}))$

\# cyclotron frequencies

wc $<-\mathrm{q} * \mathrm{~B} / \mathrm{me}$

\# diocotron frequencies

wD <- wp*wp/ $(2 * w c)$

\# evolution numbers

nev <- wD*t/(2*pi)

\# assemble data frame

9 DSET <- data.frame(fns, IH, B, V, I, NC, besttp, calx.v, caly.v, xoff.v, yoff.v, $r 1, r 2$, L, beta, $t$, lambda, $n$, wc, wp, wD, nev)

340 write.table(DSET, file=paste(out.dir, fname.id, " 386 _data_set.txt", sep=""))

\# loop over profiles

for (l in profile.list) \{

if $(B[1]==0.16)\{$

$\mathrm{X} \operatorname{corr}=\mathrm{X} \operatorname{corrl}[1]$

Ycorr $=$ Ycorrl [1]

\}

else if $(B[1]==0.24)\{$

$\mathrm{X} \operatorname{corr}=\mathrm{X} \operatorname{corr} 1[2]$

Ycorr $=$ Ycorrl [2]

\}

else if $(B[1]==0.32)\{$

$\mathrm{X} \operatorname{corr}=\mathrm{X} \operatorname{corrl}[3]$

Ycorr $=Y \operatorname{corrl}[3]$

\}

else if $(B[1]==0.4)\{$

$\mathrm{Xcorr}=\mathrm{Xcorrl}[4]$

Ycorr $=Y \operatorname{corrl}[4]$

\}

else \{

$\mathrm{Xcorr}=0$

Ycorr $=0$

\}

fname.id <- substr(fns[1],13,nchar(fns[1])-7) \# filename prefix

message ("fname $=$ ", fname.id)

graphics.off ()

if $(\mathrm{rgl} \cdot \operatorname{cur}()>0) \mathrm{rgl} \cdot \operatorname{close}()$

\# read scan data

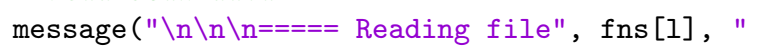
.. =====" )

cat("Hollow gun inner radius:", format(r1[1]*1 e3, di=3), "mm \n")

cat ("Hollow gun outer radius:", format(r2[1]*1 e3, di=3), "mm $\backslash n ")$

cat("Anode-collector distance:", format(L[1], $\mathrm{di}=3), \quad \mathrm{m} \backslash \mathrm{n} ")$
cat("Filament heater current:", format(IH[1], di =3), "A\n")

cat("Solenoidal field:", format(B[1]*10,di=4), "kG\n")

cat ("Cathode voltage:", format (V[1]/1e3,di=4), " $\mathrm{kV} \backslash \mathrm{n}$ ")

cat("Peak collector current:", format(I[1]*1e3, $\mathrm{di}=4), " \mathrm{~mA} \backslash \mathrm{n}$ ")

cat("Number of correctors:", format(NC[1]), "\n ")

cat ("Electron velocity:", format(v[1],di=3), "m $/ \mathrm{s}$ (beta $="$, format (beta $[1], \operatorname{di}=3), "$ ) $\backslash \mathrm{n} ")$ cat ("Flight time:", format (t [1],di=3), "s $\backslash n$ ") cat("Linear number density:", format(lambda[1], $\operatorname{di=3)}, " / \mathrm{m} \backslash \mathrm{n} ")$

cat("Volume number density:", format(n[1],di=3) , "/m3\n")

cat ("Cyclotron frequency:", format (wc[1]/(2*pi) , di=3), "Hz\n")

cat ("Plasma frequency:", format (wp [1]/(2*pi), di =3), "Hz\n")

cat("Diocotron frequency:", format (wD[1]/(2*pi) , di=3), "Hz\n")

cat("Evolution number:", format (nev[1],di=3), " rev/flight \n")

\# Finding the number of data points to plot.

message("Obtaining scan parameters...")

xraw <- round (D\$1, digits=3) \# horz. deflector , rounded

Xmin <- $\min (x r a w) ; X \max <-\max$ (xraw)

$\mathrm{Nx}<-$ nlevels (as.factor (xraw)) ; Xstep <- (Xmax$\mathrm{Xmin}) /(\mathrm{Nx}-1)$

yraw <- round(D\$V2, digits=3) \# vert. deflector , rounded

Ymin <- $\min$ (yraw); Ymax <- $\max$ (yraw)

Ny <- nlevels (as.factor (yraw)) ; Ystep <- (YmaxYmin)/(Ny-1)

\# define grid

$X<-\operatorname{seq}\left(X \min , X_{\max }\right.$, length. out=Nx)

$Y<-$ seq(Ymin, Ymax, length. out=Ny)

cat ("X scan: from", Xmin, "to", Xmax, "(", Nx, "points) $\backslash \mathrm{n}$ ")

cat ("Y scan: from", Ymin, "to", Ymax, "(", Ny, "points) \n")

\# new profiles have vacuum data in 5 th column ( ACL scripts)

VACUUM <- FALSE

if $(1>=57)$ VACUUM <- TRUE

\# calibration of $20-l / s$ Tevatron diode pump gauge

Vga <- $c(9,8.4,8,7,6.9,6,5.4,5,4,3.85,3)$ \#V

$\operatorname{logPtorr}<-\log 10(c(4 e-10,1 e-9,2 e-9,8.5 e-9$, $1 e-8,4 e-8,1 e-7,1.8 e-7,8 e-7,1 e-6,5.5 e-6)$ ) \#[torr], $\log$ scale

logPvsVsm <- smooth.spline(Vga, logPtorr)

PvsV <- function $(V=6)\{$

Vmin <- 2.8; Vmax <- 9.9

$\mathrm{P}<-1 \mathrm{e}-4+0 * \mathrm{~V}$

$\mathrm{P}[\mathrm{V}>=\mathrm{Vmax}]<-1 \mathrm{e}-10$

$\mathrm{P}[\mathrm{V}<=\mathrm{Vmin}]<-1 \mathrm{e}-4$ 
good <- Vmin<V \& V<Vmax

$\mathrm{P}$ [good] <- predict(logPvsVsm, $\mathrm{x}=\mathrm{V}$ [good]) $\$ \mathrm{y}$

$\mathrm{P}[$ good $]<-10^{\sim} \mathrm{P}[$ good] $* 1.333$ \# [mbar]

$\operatorname{return}(\mathrm{P})$

\}

message ("Defining profile matrices...")

Zprobe <- matrix (0, nrow $=\mathrm{Nx}$, ncol=Ny)

Ztube <- matrix $(0$, nrow $=\mathrm{Nx}, \mathrm{ncol}=\mathrm{Ny})$

Zvac <- matrix (NA, nrow $=\mathrm{Nx}, \mathrm{ncol}=\mathrm{Ny})$

\# assign value according to position in grid

for (k in $1:$ length $(\mathrm{D} \$ \mathrm{~V} 3))\{$

$i<-\operatorname{round}((\operatorname{xraw}[\mathrm{k}]-\mathrm{Xmin}) / \mathrm{Xstep})+1$

$j<-\operatorname{round}(($ yraw $[k]-Y m i n) /$ Ystep $)+1$

Zprobe $[i, j]<-$ D\$V3 $[k]$

Ztube $[i, j]<-D \$ 4[k]$

\# new profiles have vacuum data

\}

if (VACUUM) Zvac $[i, j]<-\operatorname{PvsV}(\mathrm{D} \$ \mathrm{VV}[\mathrm{k}])$

\# choose which variable to analyze and plot

$\mathrm{Z}<-$ Zprobe

\# correct for scallops due to raster scan?

if (CORRECT . SCALLOPS) \{

message("Correcting for scallops due to raster scan...")

\# find best correction parameter?

if (FIND . SCALLOP . CORRECTION . PAR) \{

message("Looking for best correction parameter 493 ...")

\# tpval <- seq $(-2,0$, by=0.05)

tpval <- seq(besttp[1]-0.2, besttp[1]+0.2, by

$=0.05$ )

hfc <- $0 *$ tpval

for (t in 1:length(tpval)) \{

tp $<-$ tpval [t]

Ztmp <- Z

for $(i \operatorname{in} \operatorname{seq}(1, N x))\{$

if $(f \operatorname{loor}(i / 2)==(i / 2)) \quad\{$ \# odd rows

for $(j$ in $2:(\mathrm{Ny}))\{$

$Z \operatorname{tmp}[i, j]<-Z[i, j-1]+(Z[i, j]-Z[i, j-1])$ / (1-tp) $\}\}$

else\{ \# even rows

for $(j$ in $1:(\mathrm{Ny}-1))\{$

$Z \operatorname{tmp}[i, j]<-Z[i, j+1]+(Z[i, j]-Z[i, j+1])$

/ (1-tp) \}\}\}

\# evaluate scallop frequency component

\# by looking at FFT of aligned rows if $(F)\{$

if $(\mathrm{floor}(\mathrm{Nx} / 2) \quad !=(\mathrm{Nx} / 2))\{$

$\mathrm{Zv}<-\mathrm{Ztmp}[1:(\mathrm{Nx}-1), 1]$

for $(j$ in $2: N y)\{Z v<-c(Z v, Z t m p[1 \cdot(N x-1), j$ ]) $\}\}$

else\{

$\mathrm{Zv}<-\mathrm{Ztmp}[1: \mathrm{Nx}, 1]$

for $(j \text { in 2:Ny) }\{\mathrm{Zv}<-c(Z v, Z \operatorname{tmp}[1: N x, j])\}\}_{516}$ $1 \mathrm{FZv}<-$ length $(\mathrm{Zv})$

win <- get.slepians (1FZv, 1, 3) [,1]

$\mathrm{FZv}<-\operatorname{abs}(\mathrm{fft}(\mathrm{Zv} *$ win $))-2$

centr $<-1 F Z v / 2$

hwdth $<-3$

hfc $[\mathrm{t}]<-\operatorname{sum}(\mathrm{FZv}[$ floor (centr-hwdth) : ceiling 520 (centr+hwdth)])
\}

\# by looking at spatial FFT

if $(\mathrm{T})\{$

icmtmp <- $\operatorname{sum}(t(1: \mathrm{Nx}) *$ Ztmp) / $\operatorname{sum}($ Ztmp)

jcmtmp <- $\operatorname{sum}(Z t m p * 1: N y)$ / sum(Ztmp)

scallop.filter <- abs ((1:Nx-icmtmp) \%०\% (1:

Ny-jcmtmp))

scallop.filter <- scallop.filter / $\max ($ scallop.filter)

scallop.filter <- 1 -scallop.filter

scallop.filter <- (scallop.filter $>0.8$ )

win <- get.slepians (Nx, 1,3) [,1] \% \% get.

slepians (Ny, 1, 3) $[, 1]$

FZ <- Mod (fft (Ztmp*win))

hfc[t] <- sum( FZ[scallop.filter] ) / sum(

FZ[!scallop.filter] ) \}

\# "maximum entropy"

if $(F)\{$

Zprb <- Ztmp / sum (Ztmp)

hfc [t] <- $\operatorname{sum}(Z p r b) * \log (\operatorname{sum}(Z p r b))$

for $(i$ in 1:Nx) \{ for $(j$ in 1:Ny) $\{h f c[t]<-h f c$

[t] $-\operatorname{Zprb}[i, j] * \log (\operatorname{Zprb}[i, j])\}\}$ $\mathrm{hfc}[\mathrm{t}]<--\mathrm{hfc}[\mathrm{t}]$

\}

\}

cat("Please select minimum of figure of merit (1 click) $\left.\backslash \mathrm{n}^{\prime \prime}\right)$

$\operatorname{par}(\operatorname{mar}=c(5,4,4,2)+0.1)$

plot (tpval, hfc, type="p", pch=16)

spf <- splinefun (tpval, hfc)

curve (spf (x), min(tpval), $\max (t p v a l)$, add=TRUE )

tp $<-$ locator $(1) \$ x$

\}

besttp $[1]<-$ tp

else \{

\}

tp $<-$ besttp [1]

cat("Raster-scan correction parameter:", tp, "\ $\left.\mathrm{n}^{\prime \prime}\right)$

\section{\# correct profile}

Zc $<-$ Z

for $(i$ in $\operatorname{seq}(1, N x))\{$

if $(f \operatorname{loor}(i / 2)==(i / 2)) \quad\{$ \# odd rows

for $(j$ in 2:(Ny)) \{

$Z c[i, j]<-Z[i, j-1]+(Z[i, j]-Z[i, j-1]) /(1$

-tp) $\}\}$

else\{ \# even rows

for $(j$ in 1: $(\mathrm{Ny}-1))\{$

$Z c[i, j]<-Z[i, j+1]+(Z[i, j]-Z[i, j+1]) /(1$ -tp) $\}\}\}$

$\mathrm{Z}<-\mathrm{Zc}$

\}

\# fix 250-V profile (power supply trip)?

if (T \& fns [1]==" ../Data/HG/HG_091023_775A_250V_ 303030kG_16mA.dat") \{

message("Correcting profile with HVPS trip..." )

itrip <- 63; ic <- 54; ioff <- 1; imag <- 1

jc $<-52.8$; joff $<-3$; jmag $<-1$ 


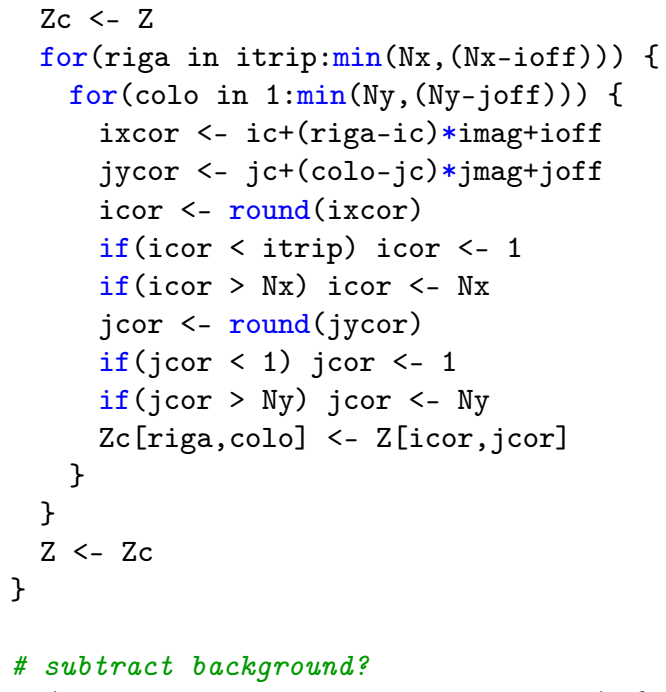

\section{.}

\section{.} ( 10 611 612

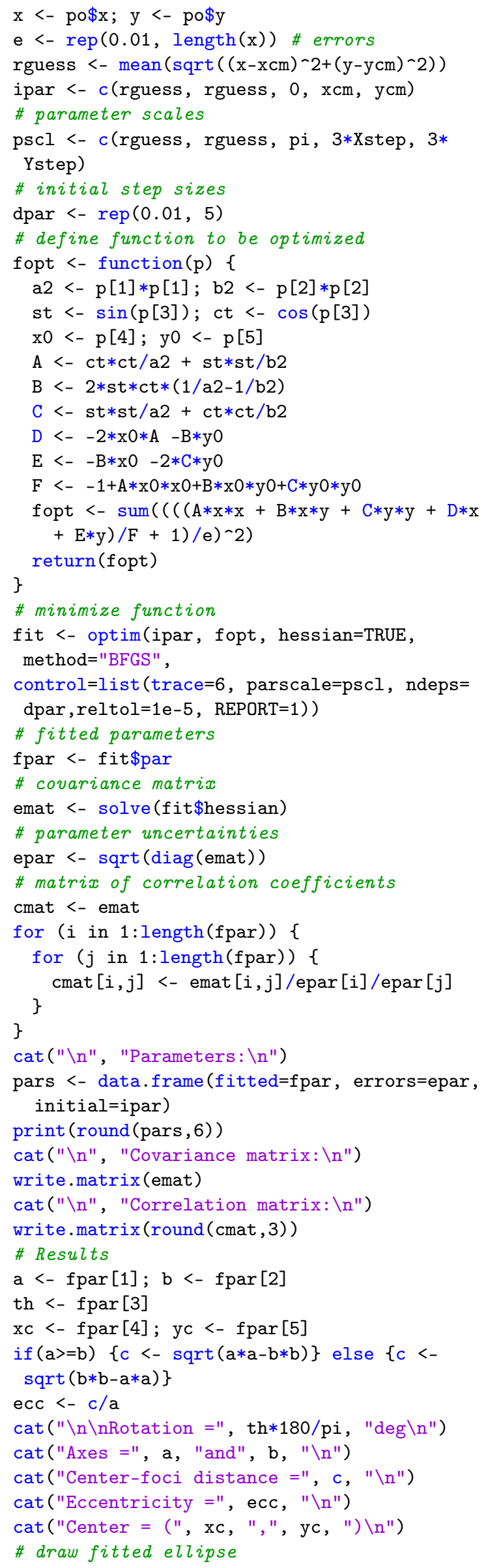




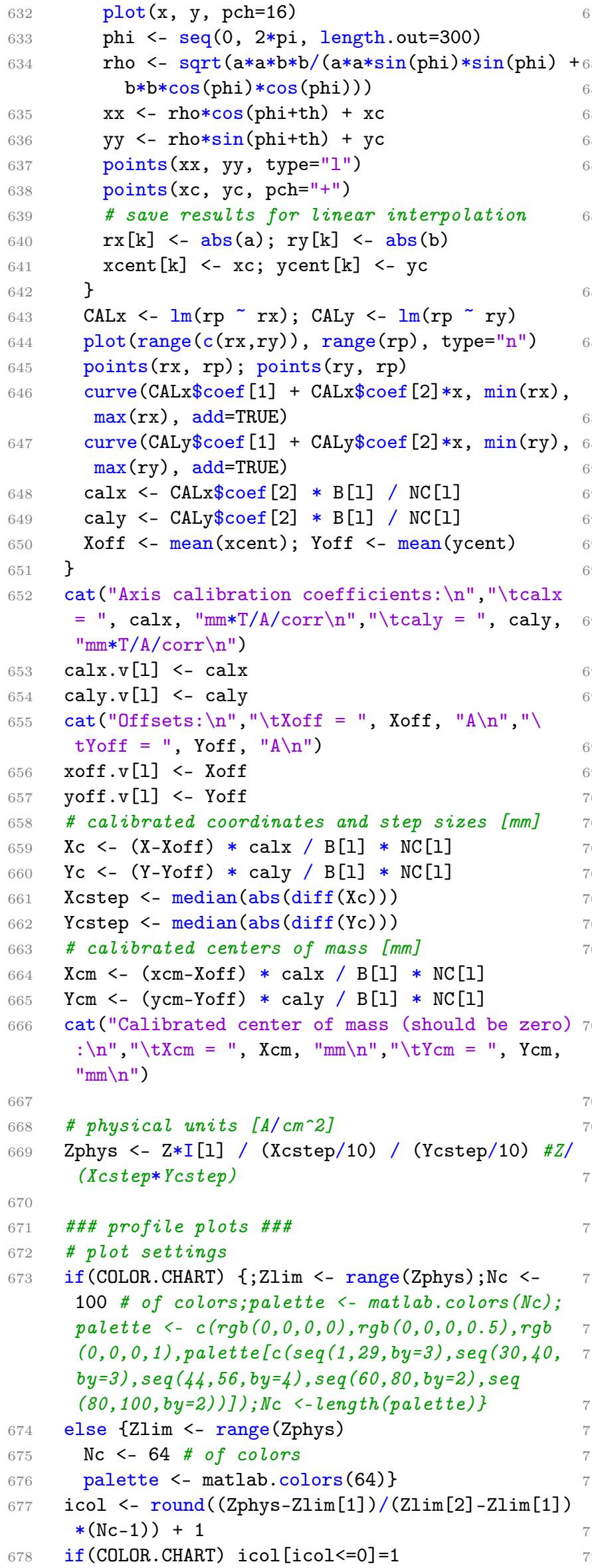

mcol <- matrix(palette[icol], nrow=Nx, ncol=Ny)

\# color matrix

\section{\# plot $1 D$ slices}

if (MAKE. PLOTS) \{

dev.new()

$\operatorname{par}($ oma $=r e p(0,4), \operatorname{mar}=c(3.5,3.5,0.5,0.5)$, $\mathrm{mgp}=\mathrm{c}(2,0.5,0), \mathrm{tcl}=-0.2)$

if (BKGR.BLACK) par ( $\mathrm{fg}="$ white", col="white", col.axis="white", col.lab="white", col.main=" white", col.sub="white")

$\mathrm{xx}<-\mathrm{Xc}$; yy <- Zphys[,jcm] \# horz. along c.m. slice

plot (xx, yy, type $=" n "$, axes=FALSE, $x l a b="$

Horizontal position [mm]",ylab=expression( paste ("Current density [", A/cm^2, "]")))

$\operatorname{axis}(1)$; $\operatorname{axis}(2, \operatorname{las}=1)$

$\operatorname{grid}($ lty="solid")

box ()

points (xx, yy, pch=16)

$f<-\operatorname{splinefun}(x x, y y)$

curve $(\mathrm{f}(\mathrm{x}), \min (\mathrm{xx}), \max (\mathrm{xx}), \mathrm{n}=301, \operatorname{add}=\mathrm{TRUE})$

file.prefix <- paste(out.dir, fname.id, "1Dx_" , sep="")

fout <- paste(file.prefix,formatC(1, width=2, flag="0"), ".pdf", sep="")

dev.print (pdf, file=fout)

fout <- paste(file.prefix, formatC(l, width $=2$, flag="0"), ".eps", sep="")

\}

dev.print (postscript, file=fout)

\# -- -2D PLOTS - - \#

if (MAKE. PLOTS) \{

\# raw $2 D$ measurement

dev.new()

$\operatorname{par}($ oma $=r e p(0,4), \operatorname{mar}=c(4.2,4.2,0.5,0.5))$

if (BKGR.BLACK) par ( $\mathrm{fg}="$ white", col="white", col.axis="white", col.lab="white", col.main=" white", col.sub="white")

image (X, Y, Zprobe, col=matlab.colors (64), asp $=1, \mathrm{xlab}=" \mathrm{H}$. corrector setting [A]",ylab="V. corrector setting [A]")

\# save to file

file.prefix <- paste(out.dir, fname.id, "raw2 $\left.D_{-} ", \quad s e p=" "\right)$

fout <- paste(file.prefix,formatC(1, width=2, flag="0"), ".png", sep="")

dev2bitmap (file=fout, width=pwid, height=phei, res=reso)

fout <- paste(file.prefix, formatC(1, width=2, flag="0"), ".pdf", sep="")

dev.print (pdf, file=fout)

fout <- paste(file.prefix, formatC(l, width $=2$, flag="0"), ".eps", sep="") \}

\# $2 D$ contours

xylim <- c(-1,1) * xylim.fudge * (r2[1]*1e3) \# plot limits [mm]

\# - - 2D CONTOUR PLOTS - - \#

if (MAKE. PLOTS) \{ 
\# plot 2D contour, bare dev.new ()

$\operatorname{par}(\operatorname{oma}=r e p(0,4), \operatorname{mar}=\operatorname{rep}(0,4))$

if (BKGR.BLACK) par (fg="white", col="white", col. axis="white", $\operatorname{col} .1 \mathrm{lab}=$ "white", $\operatorname{col}$.main=" white", col.sub="white")

filled.contour.nokey(Xc, Yc, Zphys, xlim=xylim , ylim=xylim, asp=1, levels=seq(Zlim[1], Zlim 752 [2], length.out=Nc), col=palette, frame.plot= FALSE)

\section{\# save to file}

file.prefix <- paste(out.dir, fname.id, "2 754 Dbare_", $\operatorname{sep}="$ ")

fout <- paste(file.prefix,formatC(1, width=2, flag="0"), ".png", sep="")

dev.print (png, file=fout, width=pwpx, height= phpx)

\#dev2bitmap ( $f i l e=f o u t$, width=pwid, height=phei759 , res=reso)

fout <- paste (file.prefix,formatC(l, width=2, 76 flag="0"), ".pdf", $\operatorname{sep}="$ ")

dev.print (pdf, file=fout)

fout <- paste(file.prefix,formatC(l,width=2, 764 flag="0"), ".eps", $\operatorname{sep}="$ ")

dev.print (postscript,file=fout)

if (MAKE.PLOTS) \{

\# plot 2D contour, legend,

wiland <- 7; heland <- 6

dev.new (width=wiland, height=heland)

$\operatorname{par}(\operatorname{oma}=\operatorname{rep}(0,4), \operatorname{mar}=c(4.2,3.2,4.2,0.5), \operatorname{mgp}=$

$c(1.5,0.5,0))$

if (BKGR.BLACK) par ( $\mathrm{fg="white",} \mathrm{col="white",}$ col.axis="white", $\operatorname{col} .1 \mathrm{ab}=$ "white", col.main=" 769 white", col.sub="white")

tick <- $\operatorname{diff}(\operatorname{range}(\mathrm{Xc})) * 0.015 ;$ tcol <- " 770 white"

plot.ticks <- function() \{axis(1); $\operatorname{axis}(2)$;

lines $(\mathrm{c}(\mathrm{Xcm}, \mathrm{Xcm}), \mathrm{c}(\min (\mathrm{Yc}), \min (\mathrm{Yc})+\mathrm{tick})$, col=tcol); lines $(\mathrm{c}(\mathrm{Xcm}, \mathrm{Xcm}), \mathrm{c}(\mathrm{Ycm}-\mathrm{tick}, \mathrm{Ycm}+772$ tick), col=tcol); lines $(c(X c m, X c m), c(\max (Y c)-773$ tick, $\max \left(\mathrm{Yc}_{\mathrm{c}}\right)$ ), col=tcol); lines (c(min (Xc), min ( $\mathrm{Xc})+\mathrm{tick}), \mathrm{c}(\mathrm{Ycm}, \mathrm{Ycm}), \mathrm{col}=\mathrm{tcol})$; lines $(\mathrm{c}(\mathrm{Xcm}-774$ tick, Xcm+tick), c(Ycm,Ycm), col=tcol); lines (c775 $(\max (X c)-t i c k, \max (X c)), c(Y c m, Y c m), c o l=t c o l) 776$ \}

plot.title <- function() \{title(main=bquote( 778 paste (I[fil], " = ", . $($ format $(\mathrm{IH}[1]$, nsmall 779 =2)), "A ", "B = ", . (format $(B[1] * 10, \operatorname{nsmall} 780$ =1)), " $\mathrm{kG}$ ", "V = ", . (format $(\mathrm{V}[1] / 1 \mathrm{e} 3$, nsmall=2)), " $\mathrm{kV} ", \mathrm{I}[\mathrm{e}], "$ " = ". (format (I [I] 781 *1e3, nsmall=0)), " $\left.\mathrm{mA}^{\mathrm{N}}\right)$ ), $\mathrm{xlab}=\mathrm{x}[\mathrm{mm}]$ ", ylab= "Y $[\mathrm{mm}] ")\}$

filled.contour (Xc, Yc, Zphys, xlim=xylim, ylim782 $=x y l i m, a s p=1, \operatorname{levels}=\operatorname{seq}(Z \lim [1], Z 1 i m[2]$, length. out=Nc), col=palette,plot.title=plot. 783 title(),plot. axes=plot.ticks (), key.title= title (main=expression (paste ( $j$, " [", A/ $\mathrm{cm}^{\wedge} 2$, "] "))), key $\cdot \operatorname{axes}=\operatorname{axis}(4))$ if (RUBBER. STAMP) \{mtext (paste (fns [1], "\n", " Analyzed on", date(), " ",R.version.string), side $=1$, line $=3$, adj $=1$, font $=3$, cex $=$ .7)\}
\# save to file

file.prefix <- paste(out.dir, fname.id, "2D_", $\operatorname{sep}="$ ")

fout <- paste(file.prefix,formatC(1, width=2, flag="0"), ".png", sep="")

\#dev.print(png, file=fout, width=wiland*reso, height=heland*reso)

dev2bitmap (file=fout, width=wiland, height= heland, res=reso)

fout <- paste(file.prefix,formatC(1, width=2, flag="0"), ".pdf", sep="")

dev.print (pdf, file=fout)

fout <- paste(file.prefix, formatC(1, width=2, flag="0"), ".eps", sep="")

\} dev.print (postscript,file=fout)

\#---2D CENTERED CONTOUR PLOTS ---\#

if (MAKE. PLOTS) \{

\# plot $2 D$ contour, bare, centered

dev.new()

$\operatorname{par}(\operatorname{oma}=r e p(0,4), \operatorname{mar}=r e p(0,4))$

if (BKGR.BLACK) par (fg="white", col="white", col.axis="white", $\operatorname{col} .1 \mathrm{ab}=$ "white", col.main=" white", col.sub="white")

filled.contour.nokey (Xc-Xcorr, Yc-Ycorr, Zphys

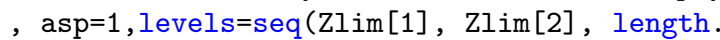
out=Nc), col=palette,frame.plot=FALSE)

\# save to file

file.prefix <- paste(out.dir, fname.id, "2

Dcentbare_", $\operatorname{sep}="$ ")

fout <- paste(file.prefix,formatC(1, width=2, flag="0"), ".png", sep="")

dev.print (png, file=fout, width=pwpx, height= phpx)

\#dev2bitmap (file=fout, width=pwid, height=phei , res=reso)

fout <- paste(file.prefix,formatC(1, width=2, flag="0"), ".pdf", sep="")

dev.print (pdf, file=fout)

fout <- paste(file.prefix, formatC(l, width=2, flag="0"), ".eps", sep="") \} dev.print (postscript,file=fout)

if (MAKE. PLOTS) \{

\# plot $2 D$ contour, legend, centered

wiland <- 7; heland <- 6

dev.new (width=wiland, height=heland)

$\operatorname{par}(\operatorname{oma}=\operatorname{rep}(0,4), \operatorname{mar}=c(4.2,3.2,4.2,0.5), \mathrm{mgp}=$ $c(1.5,0.5,0))$

if (BKGR.BLACK) par (fg="white", col="white", col. axis="white", $\operatorname{col} .1 \mathrm{ab}=$ "white", col.main=" white", $\operatorname{col}$.sub="white")

tick <- $\operatorname{diff}(\operatorname{range}(\mathrm{Xc})) * 0.015 ;$ tcol <- " white"

plot.ticks <- function() \{axis(1); axis(2); lines $(\mathrm{c}(\mathrm{Xcm}, \mathrm{Xcm}), \mathrm{c}(\min (\mathrm{Yc}), \min (\mathrm{Yc})+\mathrm{tick})$, col=tcol); lines $(\mathrm{c}(\mathrm{Xcm}, \mathrm{Xcm}), \mathrm{c}(\mathrm{Ycm}-\mathrm{tick}, \mathrm{Ycm}+$ tick $), c o l=t c o l) ; l i n e s(c(X c m, X c m), c(\max (Y c)-$ tick, $\left.\left.\max \left(Y_{c}\right)\right), \operatorname{col}=\mathrm{tcol}\right) ; \operatorname{lines}(\mathrm{c}(\min (\mathrm{Xc}), \min ($ $\mathrm{Xc})+\mathrm{tick}), \mathrm{c}(\mathrm{Ycm}, \mathrm{Ycm}), \mathrm{col}=\mathrm{tcol}) ; \operatorname{lines}(\mathrm{c}(\mathrm{Xcm}-$ tick, Xcm+tick), $c(Y \mathrm{~cm}, Y \mathrm{~cm}), \mathrm{col}=\mathrm{tcol}) ; \mathrm{lines}(\mathrm{c}$ $(\max (X c)-t i c k, \max (X c)), c(Y c m, Y c m), c o l=t c o l)$ 
\}

plot.title <- function() \{title(main=bquote( paste(I[fil], " = ", . (format (IH[I], nsmall 815 $=2)$ ), " A ", "B = ", . (format $(B[1] * 10$, nsmall =1)), " $k G$ ", "V = ", . (format $(V[1] / 1 e 3$, nsmall=2)), " kV ",I[e], " = ", . (format(I[I] *1e3, nsmall=0)), " $\mathrm{mA} ")$ ) $, \mathrm{xl} a b=" \mathrm{X}[\mathrm{mm}] ", \mathrm{ylab}=817$ "Y $[\mathrm{mm}]$ ")\}

filled.contour(Xc-Xcorr, Yc-Ycorr, Zphys,

levels=seq (Zlim[1], Zlim[2], length.out=Nc), 820 col=pplot . title=plot.tplot . axes=plot.tkey . $\quad 821$ title=title (main=expression (paste $(j$, " [", A/822 $\mathrm{cm}^{\wedge} 2$, key. $\left.\operatorname{axes}=\operatorname{axis}(4)\right)$

if (RUBBER. STAMP) \{ te (fns [1], "\n","Analyzed on", date() ${ }_{825}$ , " ",R.version.string), side $=1$, line $=3$, $\operatorname{adj}=1$, font $=3$, cex $=.7$ ) \}

\# save to file

file.prefix <- paste(out.dir, fname.id, "2 827 Dcent_", sep="")

fout <- paste(file.prefix, formatC(l, width=2, flag="0"), ".png", sep="")

\#dev.print(png, file=fout, width=wiland*reso, height=heland*reso)

dev2bitmap (file=fout, width=wiland, height= heland, res=reso)

fout <- paste(file.prefix,formatC(1, width=2, 828 flag="0"), ".pdf", sep="")

dev.print (pdf, file=fout)

fout <- paste(file.prefix, formatC(l, width=2, flag="0"), ".eps", sep="")

\}

dev $\cdot$ print (postscript, file=fout)

\#-- - 3D PLOTS ---\#

if (MAKE. PLOTS) \{

\# plot 3D

rgl.open()

\#par3d(windowRect=c(0, O, pwpxRGL, phpxRGL), 832

family="serif", cex=2.5)

par3d (windowRect=c $(0,0$, pwpxRGL, phpxRGL), 833

family="serif")

$\operatorname{bg} 3 d(p b g r)$

view3d ( fov $=45$, userMatrix $=\operatorname{matrix}(\mathrm{c}(0.7,-$

$0.7,0,0,0.54,0.54,0.57,0,-0.4,-$

$0.4,0.86,0,0,0,0,1)$, nrow $=4$, ncol $=4$, byrow $=$ TRUE) )

if (D3.AXIS) \{persp3d (Xc, Yc, Zphys, col=mcol, 83 lit=FALSE, $x l a b=" X[\mathrm{~mm}] "$, ylab="Y $[\mathrm{mm}] ", \mathrm{zlab}=$

"j $\left[\mathrm{A} / \mathrm{cm}^{\wedge} 2\right] "$, axes=TRUE, box=FALSE) $\}$

else\{persp3d(Xc, Yc, Zphys, col=mcol, lit $=839$

FALSE, $x l a b=" ", y l a b=" 1, \quad z l a b=" "$, axes=TRUE) $\}_{840}$ $\operatorname{grid3d}(c(" x+", " y+")$, at $=$ NULL, , lwd = 1, lty 841

$=1, \mathrm{n}=5)$

\#bbox3d(color=c("\#333377", "black"), emission 843

="\#333377", specular="\#3333FF", shininess $=5,844$ $a \imath p h a=0.8, x l a b=" X[\mathrm{~mm}] ", y l a b=" Y[\mathrm{~mm}] ", z l a b=" 845$ $j\left[\mathrm{~A} / \mathrm{cm}^{-2} 2\right] "$ ) \#axes3d(edges="bbox", labels = TRUE, tick =

TRUE, nticks = 5, main="Profile", $x l a b=" X[\mathrm{~mm}$

]", $\left.y \imath a b=" Y[\mathrm{~mm}] ", \quad z l a b=" j\left[\mathrm{~A} / \mathrm{cm}^{-2}\right] "\right)$

$\# b o x 3 d()$ \#title3d(main = "Profile", sub = NULL, $x l a b=$

"X", ylab= "Y", zlab= "Z", line = NA)

file.prefix <- paste(out.dir, fname.id, "3D_", sep=" ")

fout <- paste(file.prefix,formatC(1, width=2, flag="0"), ".png", sep="")

\}

if (MAKE. PLOTS) \{

\# 2D vacuum measurement

if (VACUUM) \{

\#Plim $<-c(1 e-8,1.5 e-7)$

Plim <- range (Zvac, na.rm=TRUE)

dev.new (width=wiland, height=heland)

$\operatorname{par}($ oma $=\mathrm{c}(0,0,0,1), \operatorname{mar}=\mathrm{c}$

$(4.2,3.2,4.2,0.5), m g p=c(1.5,0.5,0))$

if (BKGR.BLACK) $\operatorname{par}(\mathrm{fg}=$ "white", col="white",

col.axis="white", col.lab="white", col.main=

"white", col.sub="white")

plot.title <- function() \{title(main=bquote( paste(I[fil], " = ", . (format (IH[I], nsmall $=2)$ ), " A ", "B = ", . (format $(B[1] * 10$, nsmall=1)), " $k G$ ", "V = ", . (format $(V[1] / 1$ e3, nsmall=2)), " $k V$ ",I[e], " = ", . ( format (I[l]*1e3, nsmall=0)), " $m A ")), x l a b="$ $\mathrm{H}$ corrector setting $[\mathrm{A}] ", \mathrm{ylab}=" \mathrm{~V}$ corrector setting $[\mathrm{A}]$ ")\}

filled. contour (X, Y, Zvac, asp=1, levels $=$ seq ( $\mathrm{Plim}$ [1], Plim[2], length.out=Nc), col= palette,plot.title=plot.title ()$, \mathrm{key} \cdot \mathrm{title}=$ title (main=expression (paste (P, "[mbar]"))) )

if (RUBBER . STAMP) \{mtext (paste (fns [l] , "\n", " Analyzed on", date(), " ",R.version.string) , side $=1$, line $=3$, adj $=1$, font $=3$, cex $=.7)\}$

\# save to file

file.prefix <- paste(out.dir, fname.id, "vac2D_", sep=" ")

fout <- paste(file.prefix,formatC(1, width $=2$, flag="0"), ".png", sep="")

\#dev.print (png, file=fout, width=wiland*reso , height=heland*reso)

dev2bitmap (file=fout, width=wiland, height= heland, res=reso)

fout <- paste(file.prefix,formatC(1, width $=2$, flag="0"), ".pdf", sep="")

dev.print (pdf, file=fout)

fout <- paste(file.prefix,formatC(1, width

$=2$, flag="0"), ".eps", sep="")

\}

dev.print (postscript, file=fout)

\}

if (EXPORT . CALIBRATED. PROFILE) \{

message("Exporting calibrated profile...")

Xmat <- Xc \%०\% rep (1, Ny)

Ymat $<-\operatorname{rep}(1, \mathrm{Nx}) \%$ \% Yc

Zmat <- Zphys

CalProf <- data.frame (x.mm = as.vector (Xmat), $\mathrm{y}$ . $\mathrm{mm}=$ as.vector (Ymat), $\mathrm{j} \cdot \mathrm{Acm} 2=$ as.vector ( Zphys))

fout <- paste(out.dir, fname.id, "_",formatC(1 , width=2, flag="0"), "_calprof.txt", sep="") 
write.table(CalProf, file=fout, row.names=

FALSE, col. names=TRUE)

system(paste("gzip", fout))

if (EXPORT.RADIAL) \{ message("Calculating average radial profile...898 ")

Xmat <- (Xc-Xcorr) $\%$ \% rep (1, Ny)

Ymat <- $\operatorname{rep}(1, \mathrm{Nx}) \%$ \% (Yc-Ycorr)

$r<-$ as.vector (sqrt (Xmat^2 + Ymat^2))

rstep <- $\max$ (Xcstep, Ycstep) /4

$r f<-\operatorname{cut}(r, \operatorname{breaks}=\operatorname{seq}(\min (r), \min (r 2[1] *$

1500, $\max (r))$, by=rstep), ordered=TRUE)

$j<-$ as.vector(Zphys)

$r m<-$ tapply $(r, r f$, mean $)$

jm $<-$ tapply $(j, r f$, mean $)$

rpro <- data.frame ( $r . m m=r m, j . a u=j m)$

message("Plotting radial profile...")

dev.new ()

$\operatorname{par}($ oma $=r e p(0,4), \operatorname{mar}=c(3.2,3.2,0.5,0.5), 908$ $\operatorname{mgp}=c(1.5,0.5,0))$

plot (rm, jm, xlab="Radial distance [mm]", ylab909 ="Current density [arb. units]",pch="+", col=" blue")

file.prefix <- paste(radial.dir, fname.id, "_"911 , formatC(l, width=2, flag="0"), "_radial", $\operatorname{sep}="$ ")

fout <- paste(file.prefix, ".pdf", sep="")

dev.print (pdf, file=fout)

message("Exporting radial profile to text file913 ...")

fout <- paste(file.prefix, ".txt", sep="") 914 write.table (rpro, file=fout, row.names=FALSE, 915 col. names=TRUE)

\}

\# downsample a large array for plotting purposes

\# function returns ordered vector of indices 92 idwnsmpl <- function( $\mathrm{x}=\operatorname{runif}(100), \mathrm{n}=10)\{\mathrm{i}<-\quad 922$ $\operatorname{sort}(\operatorname{sample}(x=1: \operatorname{length}(x), \operatorname{size}=n)) ; \operatorname{return}(i)\} 923$

\# export particle coordinates

if (EXPORT.PARTICLES) \{

message("Exporting particle coordinates for 926

Warp field solver...")

Znorm $<-$ Z/sum(Z)

$\mathrm{Np}<-$ round (Nmax*Znorm)

cat ("Max \# of particles p $n ")$

cat ("Empty bins:", length (Np [Np==0]), "\n") 932

xpart <- numeric(Nmax); xpart <- NA

ypart <- numeric(Nmax); ypart <- NA

$\mathrm{k}<-1$

for $(i$ in $1: N x)\{$

for $(j$ in $1: \mathrm{Ny})\{$

if $(\mathrm{Np}[\mathrm{i}, j]>=1 \& \mathrm{k}<=\operatorname{Nmax})\{$

for (m in $1: \mathrm{Np}[i, j])\{$

\# generate coordinates uniformly within 937 each bin

$\operatorname{xpart}[\mathrm{k}]<-\mathrm{Xc}[\mathrm{i}]+\operatorname{runif}(1,-0.5 * \mathrm{Xcstep}, 938$ $0.5 *$ Xcstep) ypart $[\mathrm{k}]<-\mathrm{Yc}[j]+\operatorname{runif}(1,-0.5 *$ Ycstep $0.5 *$ Ycstep)

$\mathrm{k}<-\mathrm{k}+1$

\}\}

\}\}

cut <- $($ is .na $($ xpart $)==$ FALSE $)$ \& $($ is $. n a($ ypart $)==$ FALSE)

xpart <- xpart[cut]; ypart <- ypart [cut]

part_coord <- data.frame (xpart, ypart)

dev.new ()

$\operatorname{par}($ oma $=r e p(0,4), \operatorname{mar}=c(3.2,3.2,0.5,0.5)$, $\operatorname{mgp}=c(1.5,0.5,0))$

N.plot.max <- 4096

if (length (xpart) > N.plot.max) ii <- idwnsmpl( $\mathrm{x}=\mathrm{xpart}, \mathrm{n}=\mathrm{N} \cdot \mathrm{plot} \cdot \max )$

else ii <- 1:length (xpart)

plot (xpart [ii], ypart [ii], pch=".", asp=1,xlab

$=" X$ coord. [mm]", ylab="Y coord. [mm]")

message("Saving Particle Plot")

file.prefix <- paste(out.dir, fname.id, "_", sep=" ")

fout <- paste(file.prefix,formatC(1, width=2,

flag="0"), "_particles.eps", sep="")

dev.print (postscript, file=fout)

message("Writing coordinates of ", length(

xpart), " particles...")

fnpart <- paste(file.prefix,formatC( 1 , width

$=2$, flag="0"), "_", length(xpart), "particles.txt", sep="")

write.table (part_coord, file=fnpart, row. names $=$ FALSE, col. names=FALSE)

\}

system(paste("gzip", fnpart))

\# polar decomposition

if (POLAR. DECOMPOSITION) \{

dev. new (width=9, height $=7$ )

source(". ./Polar/polar_decomposition.R")

$\mathrm{nn}<-1: 32$ \#Was 128

$\mathrm{mm}<-0: 32$ \#Was 48

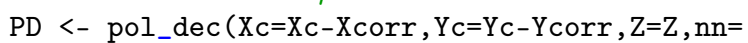
$\mathrm{nn}, \mathrm{mm}=\mathrm{mm}, \mathrm{a}=30$, BOUNDARY $=0$ )

P_mat <- PD\$P

\#rownames $\left(P_{-}\right.$mat $)<-1: 32$

\#colnames ( $P_{-}$mat $)<-0: 32$

nModes <- rowSums (Mod (P_mat))

mModes <- colSums (Mod (P_mat))

I_mat <- PD $\$$ I

\section{\# plots}

$\operatorname{par}($ oma $=c(0,0,3,0))$

a <- layout (matrix $(c(1,4,2,5,3,6), 2,3)$, widths $=$ $c(0.9,0.9,0.9))$

layout.show (a)

image (Xc-Xcorr, Yc-Ycorr, Z, asp=1, col=palette, main="Measurement")

image (Xc-Xcorr, Yc-Ycorr, Mod (I_mat), asp=1, col= palette,main="Reconstruction")

image (Xc-Xcorr, Yc-Ycorr, abs (Mod (I_mat) -Z) , asp

$=1, \operatorname{col}=\operatorname{gray}(0: 64 / 64)$, main="Errors")

ticks <- c(0.00001,

$0.0001,0.0001,0.001,0.01,0.10)$ 
image.plot (nn,mm,log10(Mod(P_mat)), col=heat. 949 colors (64), main="Mode amplitudes", $\mathrm{xlab}=" \quad 950$ Radial mode $\mathrm{n}$ ",ylab="Azimuthal mode $\mathrm{m}$ ",nlevel 951 $=64$, axis.args=list $($ at $=\log 10($ ticks $)$, labels $=$ ticks)) plot (nn, nModes, type="o", log="y",pch=16, main="953 Radial modes", xlab="Radial mode $n ", y l a b=" 954$ Amplitude")

plot (mm, mModes, type="o" , log="y",pch=16, main=" Azimuthal modes", $\mathrm{xlab}=$ "Azimuthal mode m", $\mathrm{ylab}=$ "Amplitude") mtext (fname.id, outer=TRUE)

dev.print (pdf,file="polar.pdf", width=9, height $=7$, pointsize=10,family="Times") layout (1)

file.prefix <- paste(polar.dir, fname.id, "_", $\operatorname{sep}="$ ")

\# save coefficients

fncoe <- paste(file.prefix,

formatC(l,width=2, flag="0"), ".RData", sep="966 $\operatorname{sink()}$ save (P_mat, file=fncoe)

\# save log

fnlog <- paste (file.prefix,formatC(l, width $=2$, flag="0"), ".txt", sep="")

cmnd <- paste("mv pol_dec_log.txt", fnlog)

system (cmnd)

\# save figures

fnfig <- paste (file.prefix, formatC(1, width=2 flag="0"), "_polar", ".pdf", sep="")

cmnd <- paste("cp polar.pdf", fnfig)

\} system (cmnd)

\section{\}}

960 if (MAKE.PLOTS) dev.off()

961 quartz.options (reset=TRUE)

pdf .options (reset=TRUE)

ps.options (reset $=$ TRUE)

cat ("\nStop: ", date (),"\n")

\section{C.2 Transverse Field Measurement}

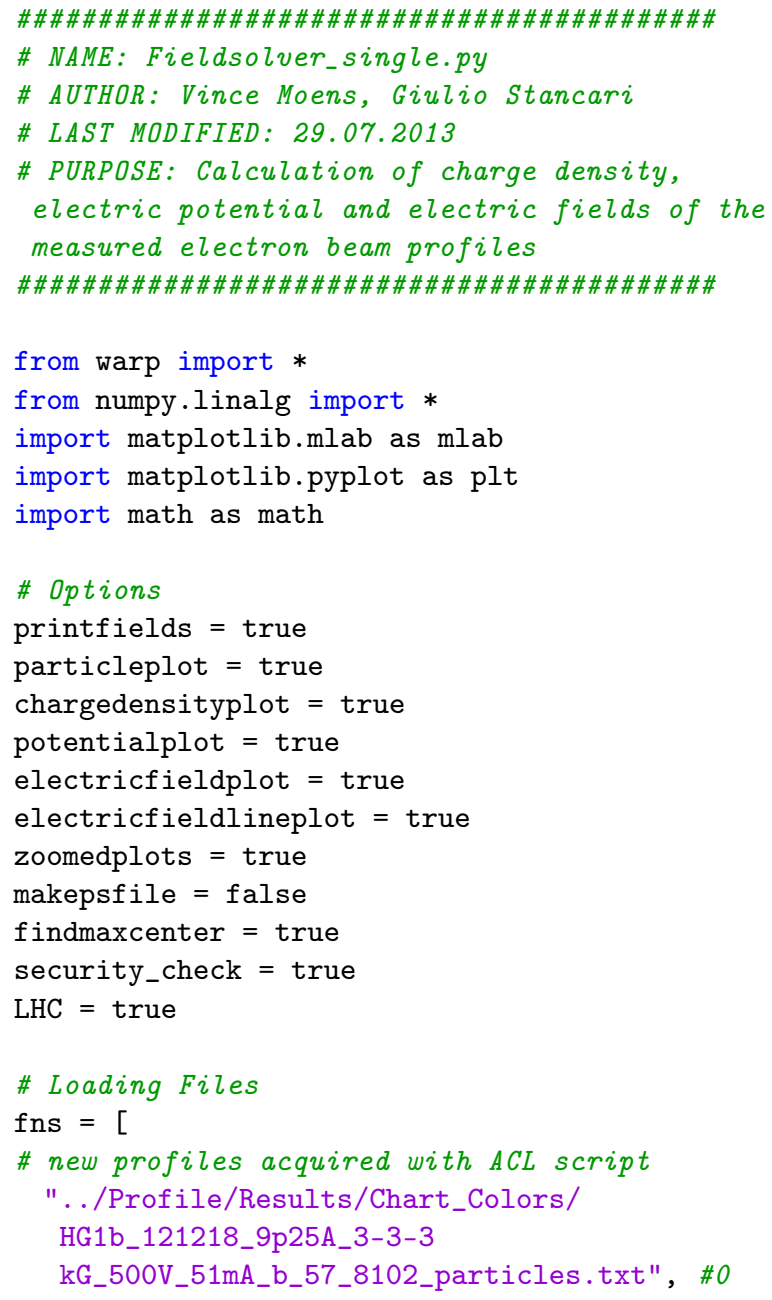

95 "...Profile/Results/Chart_Colors/ HG1b_130521_9p25A_06-24-06

98 Bgun $=[3,3,3,3,3,3,3,3,3,3,1,1,1,2.5$, $2,2.5,2.5,2.5,2.5,2,2,2,1.5,1.5,1.5$, $3,2.5,3,3,3,3,3,1,1,0.8,0.8,0.8,0.8$, $0.8,0.8,0.8,0.8,0.8,0.8,0.8,1,1,1$, $0.6,0.4,0.4,1,1,1,0.6,1,0.4,1,0.4$, $0.4,0.4,0.6,0.6,0.6]$

99 Bmain $=[3,3,3,3,3,3,3,3,3,3,1,1,1$, $2.5,2,2.5,2.5,2.5,2.5,2,2,2,1.5,1.5$, $1.5,3,2.5,3,3,3,3,3,3,3,3.2,3.2,3.2$, $3.2,3.2,3.2,3.2,3.2,3.2,3.2,3.2,4,4$, $4,2.4,1.6,1.6,4,4,4,2.4,4,1.6,4,1.6$, $1.6,1.6,2.4,2.4,2.4]$

$100 \mathrm{Bcoll}=[3,3,3,3,3,3,3,3,3,3,1,1,1$, $2.5,2,2.5,2.5,2.5,2.5,2,2,2,1.5,1.5$, $1.5,3,2.5,3,3,3,3,3,3,2,0.8,0.8,0.8$, $0.8,0.8,0.8,0.8,0.8,0.8,0.8,0.8,1,1$, $1,0.6,0.4,0.4,1,1,1,0.6,1,0.4,1,0.4$, $0.4,0.4,0.6,0.6,0.6]$

101

102 Voltage $=[500,8000,4000,2000,1000,6000,3000$, $7000,8000,8000,889,2000,3000,5556,3556$, $5000,2083,3472,5000,1333,2222,3111,750$, $2000,1250,3000,500,500,500,500,500,500$, $500,500,500,2000,4000,1000,8000,7000$, $250,125,3000,5000,6000,3125,1000,6250$, $500,1000,500,500,2000,4000,1000,5000$, $2000,8000,750,1500,2500,2000,3000,4000]$

103 Current $=[0.051,2.940,1.100,0.388,0.140$, $1.924,0.694,2.394,0.440,0.027,0.118,0.398$, $0.726,1.770,0.928,1.484,0.412,0.865$, $1.460,0.213,0.448,0.744,0.090,0.390,0.192$, $0.582,0.051,0.050,0.050,0.050,0.050$, 


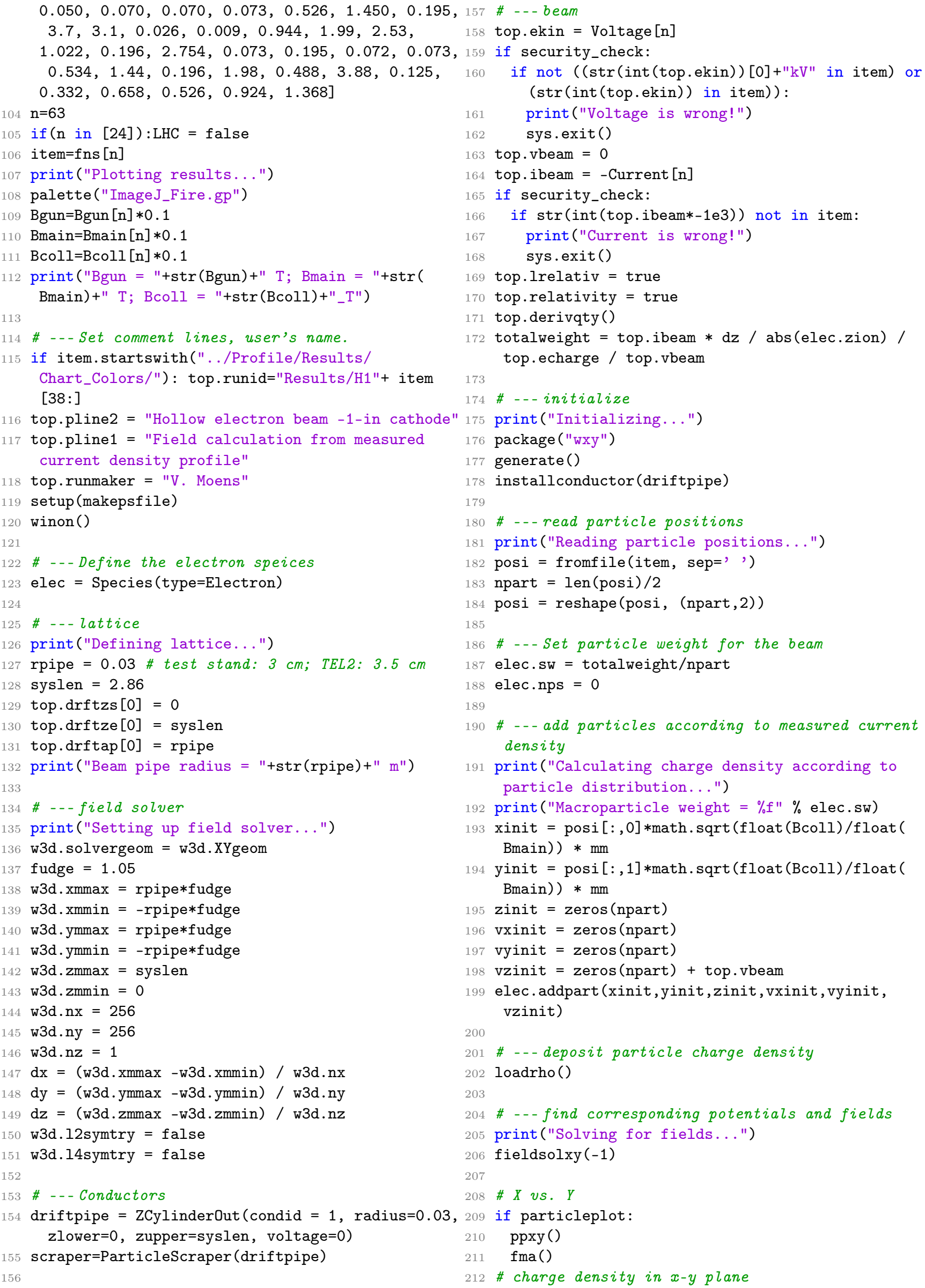




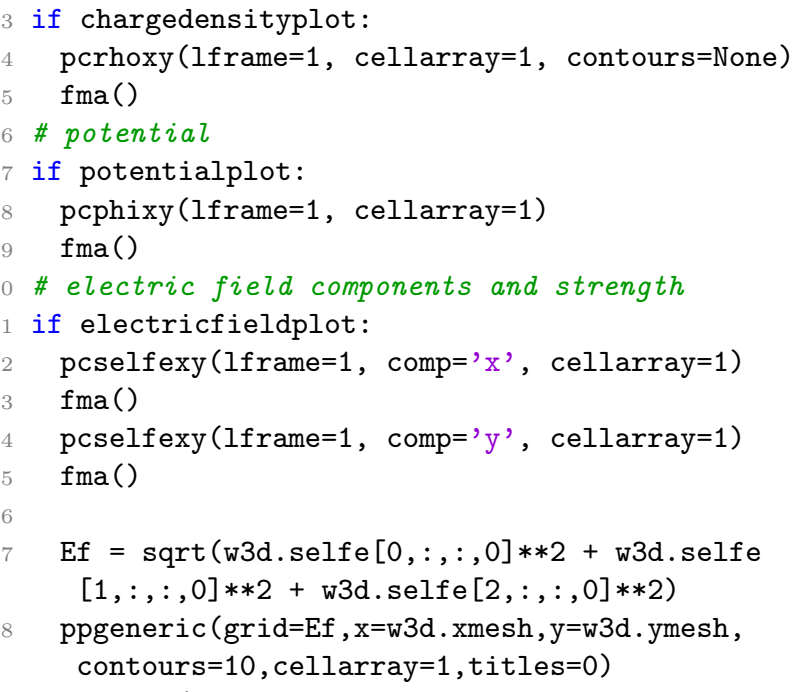

for $i$ in range(i1, i2):

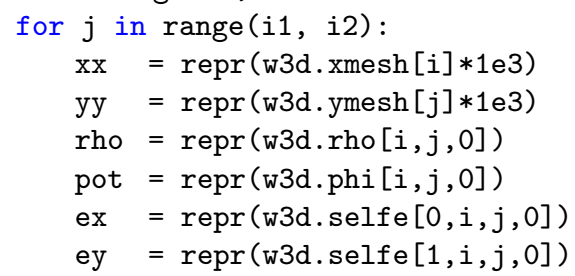

297 \#Calculating emittance growth at center

307 Er_center_6=Efcw_RMS* (6.75e-3*math.sqrt (float ( Bcoll)/float (Bmain)) /(6*sigma))

308 print "Maximum electric field in center is "+str( Er_center)+" V/m"

$309 \mathrm{~L}=2.65$

310 print "Length of interaction region is "+str(L)+" $\mathrm{m} "$

311 gamma $=$ Etot $/ \mathrm{m} 0$

312 print "gamma is "+str (Etot/m0)

313 if (LHC): beta $=250$

314 else: beta $=150$

315 print "beta is "+str(beta) 


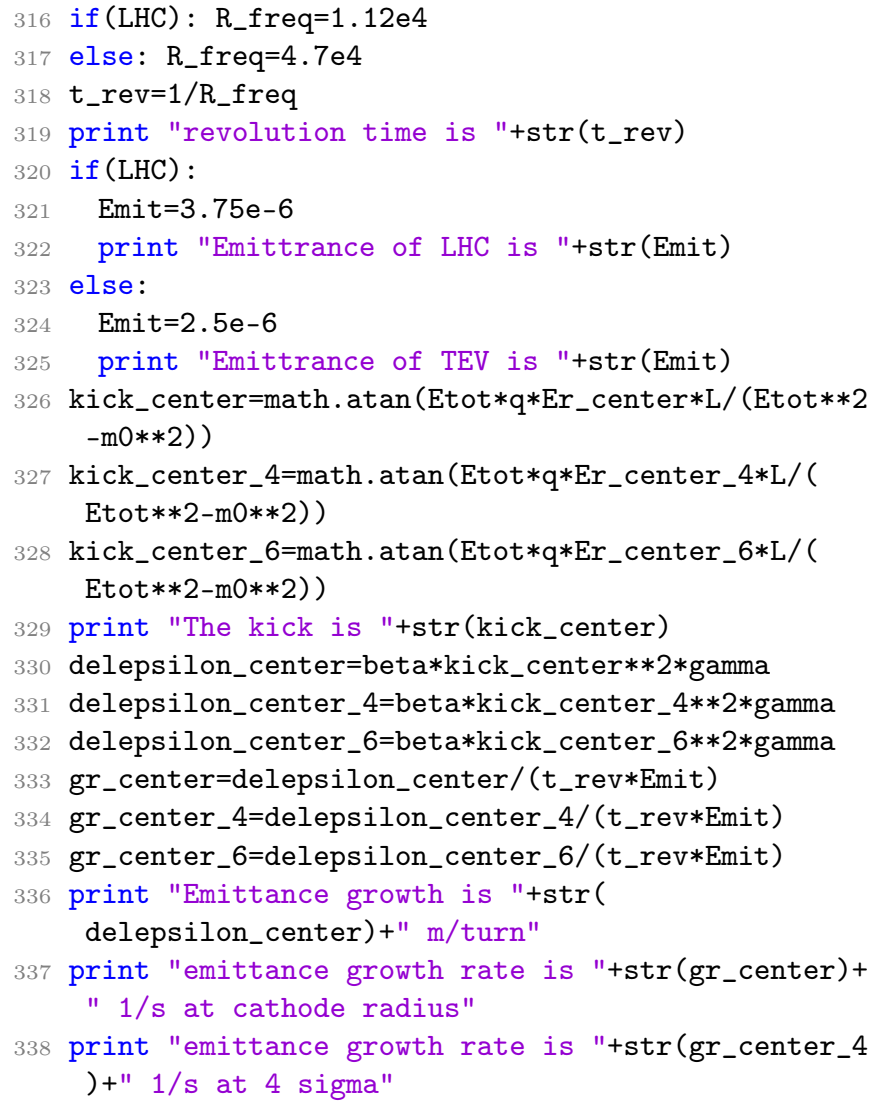

339 print "emittance growth rate is "+str(gr_center_6 )$+" 1 / \mathrm{s}$ at 6 sigma"

340 print " $\backslash \mathrm{n}$ "

341 print str (item[38:-4])

342 print " $\backslash \mathrm{n}$ Calculating Emittance Growth overall \n

343

344 name $=$ "Results/H1"+item[38:-4]+"_Emax_Gaussian. txt"

345 print "Saving data to text file: "+str(name)+" \n" out $=$ open (name, 'wb')

347 titleline $=$ item $[38:-4]+" \backslash$ tEmax $[\mathrm{kV} / \mathrm{m}] \backslash$ tKick []$\backslash$ tEmittance Growth [m/turn] tEmittance Growth Rate $[1 / \mathrm{s}] \backslash \mathrm{n} "$

348 out.write(titleline)

349 line = "Center: \t"+str (Er_center $)+" \backslash t "+\operatorname{str}($ kick_center)+" $\backslash t "+s t r($ delepsilon_center) +" $\backslash t "+$ $\operatorname{str}\left(g r_{-}\right.$center) $+" \backslash \mathrm{n} "$

350 out.write(line)

351 line $=$ "Center_6s: $\backslash \mathrm{t} "+\operatorname{str}($ Er_center_6)+"\t"+str ( kick_center_6)+"\t"+str (delepsilon_center_6)+"\t "+str (gr_center_6)+"\n"

352 out.write(line)

353 line = "Center_4s: $\backslash t "+\operatorname{str}\left(E_{-}\right.$center_4) +" $t t "+s t r($ kick_center_4)+"\t"+str(delepsilon_center_4)+"\t "+str (gr_center_4)+" $\backslash \mathrm{n} "$

354 out.write(line) out.close()

Attachments/Fieldsolver_single_app.py

\section{C.3 Numerical WARP simulations}

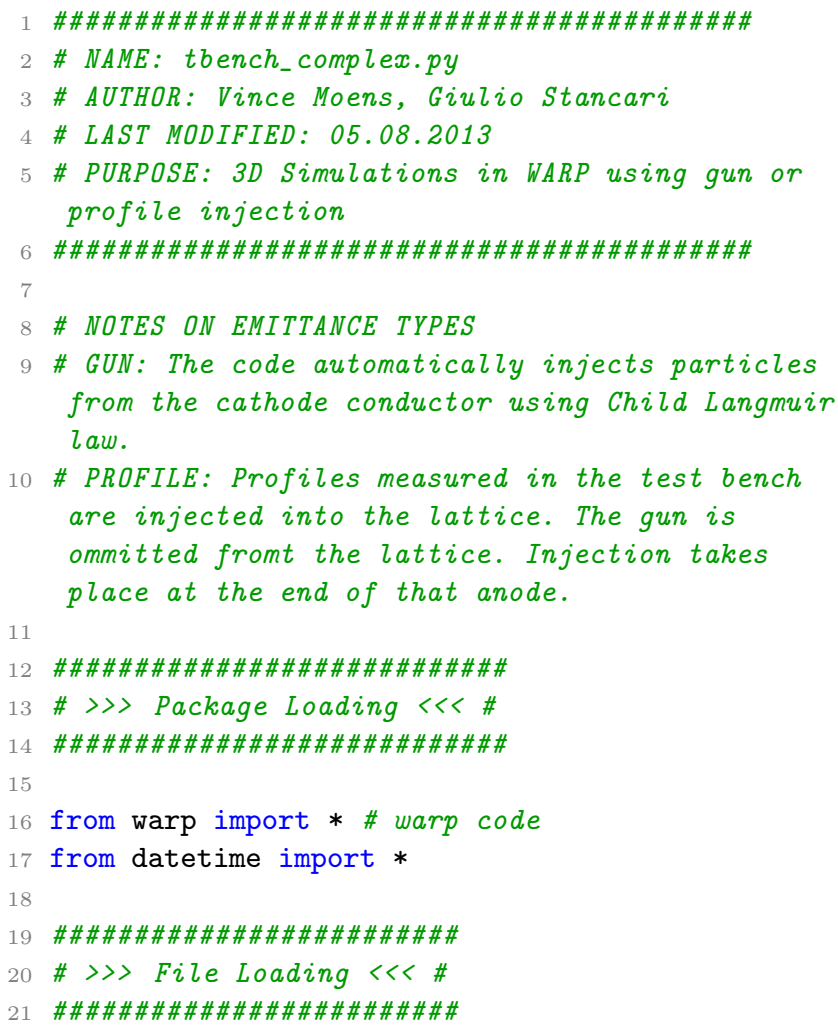

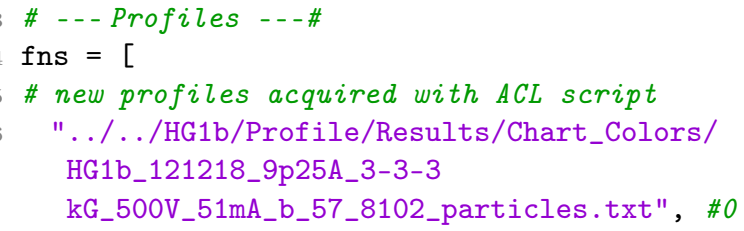


96 Bcoll $=[3,3,3,3,3,3,3,3,3,3,1,1,1$, $2.5,2,2.5,2.5,2.5,2.5,2,2,2,1.5,1.5$, $1.5,3,2.5,3,3,3,3,3,3,2,0.8,0.8,0.8,13$ $0.8,0.8,0.8,0.8,0.8,0.8,0.8,0.8,1,1$, $1,0.6,0.4,0.4,1,1,1,0.6,1,0.4,1,0.4$, $0.4,0.4,0.6,0.6,0.6]$

98 Voltage $=[500,8000,4000,2000,1000,6000,3000$ $7000,8000,8000,889,2000,3000,5556,3556$, $5000,2083,3472,5000,1333,2222,3111,750$, $2000,1250,3000,500,500,500,500,500,500$, $500,500,500,2000,4000,1000,8000,7000$, $250,125,3000,5000,6000,3125,1000,6250$, $500,1000,500,500,2000,4000,1000,5000$, $2000,8000,750,1500,2500,2000,3000,4000]$

99 Current $=[0.051,2.940,1.100,0.388,0.140$, $1.924,0.694,2.394,0.440,0.027,0.118,0.398$, $0.726,1.770,0.928,1.484,0.412,0.865$, $1.460,0.213,0.448,0.744,0.090,0.390,0.192$, $0.582,0.051,0.050,0.050,0.050,0.050$, $0.050,0.070,0.070,0.073,0.526,1.450,0.195$, $3.7,3.1,0.026,0.009,0.944,1.99,2.53$, $1.022,0.196,2.754,0.073,0.195,0.072,0.073$, $0.534,1.44,0.196,1.98,0.488,3.88,0.125$, $0.332,0.658,0.526,0.924,1.368]$

1 \#---Selecting profile ---\#

item $=51$

file_ending = "test"

Bmain $=$ float $($ Bmain $[$ item $]) / 10$

print("Magetic Field in Main Solenoid: \%g " \% Bmain)

106 Bgun = float $($ Bgun $[$ item $]) / 10$

$107 \mathrm{Bcoll}=$ float $(\mathrm{Bcoll}[$ item $]) / 10$

08 Cathode_Potential = -Voltage $[$ item $]$

09 print("Cathode Potential: \%g " \%

Cathode_Potential)

110 Current = Current [item]

print ("Current: \%g " \% Current)

npart $=-50 *$ Cathode_Potential $/ 500$

\section{\#\#\#\#\#\#\#\#\#\#\#\#\#\#\#\#}

\# $>>$ Options $<<$ \#

\section{\#\#\#\#\#\#\#\#\#\#\#\#\#\#\#\#}

machine_injtype = "profile"

print("Injection type is:"+machine_injtype)

if (machine_injtype=="gun"): machine_emittype $=2$

20 elif (machine_injtype=="profile"):

machine_emittype $=1$

21 else:

print("Wrong machine_injtype!!")

quit ()

machine_type = "tbench"

\#\#\#\#\#\#\#\#\#\#\#\#\#\#\#\#\#

$\#>>$ Headers $<<$ \#

\#\#\#\#\#\#\#\#\#\#\#\#\#\#\#\#

now=datetime.now ()

date=now.strftime $(11 \% \mathrm{y} \% \mathrm{~m} \% \mathrm{~d} ")$

time=now.strftime ( $1 \% \mathrm{H} \% \mathrm{M} ")$

if not os.path.exists("../Results/"+date+"/"): os.makedirs (". ./Results/"+date+"/")
134 os.system("cp tbench_complex.py* ../Results/"+ date+"/tbench_"+date+time+"_test.py")

top.runid = machine_type+"_"+date+time+"_"+ machine_injtype+"_"+file_ending

36 if machine_type == "tbench": top.pline $2=$ " Electron Lens Test Bench"

137 else: top.pline2 = "Tevatron Electron Lens 2" if machine_emittype $==1$ : top.pline $1=$ "Constant injection_" + machine_injtype

139 elif machine_emittype $==2$ : top.pline1 = "ChildLangmuir_" + machine_injtype

140 else: top.pline1 = "other injection method"

top.runmaker $=$ "V. Moens"

142

\section{\#\#\#\#\#\#\#\#\#\#\#\#\#\#\#\#\#}

\# $>>$ Variables $<<\#$

\section{\#\#\#\#\#\#\#\#\#\#\#\#\#\#\#\#\#}

\#---Machine Parameters -.-\#

machine_zstart $=.0 \mathrm{e} 0$

machine_syslen $=2.86$

machine_zplat $=$ machine_syslen

machine_piperad $=3 * \mathrm{~cm}$

zfinal = machine_zstart + machine_syslen

\#---Electron Gun -.-\#

\# - Cathode

Cathode_zstart $=-29.25 * \mathrm{~mm}$

Cathode_zend $=0.0 * \mathrm{~mm}$

Cathode_radi $=6.75 * \mathrm{~mm}$

Cathode_rado $=12.7 * \mathrm{~mm}$

Cathode_radcurvb $=10 * \mathrm{~mm}$

Cahtode_radcurvs $=0.5 * \mathrm{~mm}$

Cathode_voltage $=$ Cathode_Potential

\# - Anode

Anode_zstart $=9.48 * \mathrm{~mm}$

4 Anode_z1 = Anode_zstart $+1.5 * \mathrm{~mm}$

Anode_z2 $=$ Anode_zstart $+3.5 * \mathrm{~mm}$

Anode_z3 $=$ Anode_z1 $+9 * m m$

7 Anode_z4 $=$ Anode_z3 $+11.25 * \mathrm{~mm}$

Anode_z5 $=$ Anode_z4 + 5.625 $* \mathrm{~mm}$

Anode_zend $=$ Anode_z5 $+58.5 * \mathrm{~mm}$

Anode_ri $=14.25 * \mathrm{~mm}$

Anode_ro $=$ Anode_ri+5.33 $* \mathrm{~mm}$

Anode_r1 = Anode_ri

Anode_r2 = Anode_ro

Anode_r3 = Anode_ri

Anode_r4 = Anode_ri $+0.675 * \mathrm{~mm}$

6 Anode_radtipi $=$ Anode_ri $+1.5 * \mathrm{~mm}$

Anode_radtipo $=$ Anode_ro $-3.5 * \mathrm{~mm}$

Anode_r5 = Anode_ri + 5.625 $* \mathrm{~mm}$

9 Anode_rendi $=$ Anode_r5

80 Anode_rendo $=$ Anode_rendi $+1.35 * \mathrm{~mm}$

Anode_radcurvb $=3.5 * \mathrm{~mm}$

Anode_radcurvs $=-1.5 * \mathrm{~mm}$

Anode_voltage $=0.0 \mathrm{e} 0$

\#-Electrode $F$

85 ElectrodeF_zstart = Cathode_zstart

ElectrodeF_zend $=0.98 * \mathrm{~mm}$

87 ElectrodeF_z1 = ElectrodeF_zend $-0.5 * \mathrm{~mm}$

188 ElectrodeF_z2 = ElectrodeF_zend $-1.4 * \mathrm{~mm}$

189 ElectrodeF_ri $=13.1 * \mathrm{~mm}$

190 ElectrodeF_ro $=$ ElectrodeF_ri $+1.9 * \mathrm{~mm}$

191 ElectrodeF_r1 = ElectrodeF_ri $+0.5 * \mathrm{~mm}$ 
192 ElectrodeF radcurvs $=-0.5 * \mathrm{~mm}$

ElectrodeF_radcurvb $=1.4 * \mathrm{~mm}$

ElectrodeF_voltage $=$ Cathode_Potential

\#- Electrode C

ElectrodeC_zstart $=$ Cathode_zstart

ElectrodeC_zend $=1.97 * \mathrm{~mm}$

ElectrodeC_ri $=20.5 * \mathrm{~mm}$

ElectrodeC_ro $=22.0 * \mathrm{~mm}$

ElectrodeC_radcurv $=0.75 * \mathrm{~mm}$

ElectrodeC_z1 = ElectrodeC_zend-0.75*mm

ElectrodeC_voltage $=$ Cathode_Potential

\# - Gun drift pipe

Gun_pipe_zstart $=84.375 * \mathrm{~mm}$

Gun_pipe_zend $=178.875 * \mathrm{~mm}$

Gun_pipe_ri $=36 * \mathrm{~mm}$ \#Should this not be $3 \mathrm{~cm}$ ?

Gun_pipe_ro $=33.75 * \mathrm{~mm}$

Gun_pipe_voltage $=0.0$

\#--.-Solenoids -.-\#

\# - Gun Solenoid

tbench_solenoid_gun_zstart $=-13 * \mathrm{~cm}$

3 tbench_solenoid_gun_zend $=37 * \mathrm{~cm}$

tbench_solenoid_gun_radi $=28 * \mathrm{~cm}$

5 tbench_solenoid_gun_rado $=$

tbench_solenoid_gun_radi $+5.433 * \mathrm{~cm}$

tbench_solenoid_gun_b = Bgun

tbench_solenoid_gun_voltage $=0.0$

\# - Main Solenoid

tbench_solenoid_main_zstart $=0.60$

tbench_solenoid_main_zend $=2.52$

tbench_solenoid_main_radi $=0.20$

tbench_solenoid_main_rado $=$

tbench_solenoid_main_radi $+14.48 * \mathrm{~cm}$

tbench_solenoid_main_b = Bmain

tbench_solenoid_main_voltage $=0.0$

\# - Collector Solenoid

tbench_solenoid_col_zstart $=2.67$

tbench_solenoid_col_zend $=3.17$

tbench_solenoid_col_radi $=28 * \mathrm{~cm}$

tbench_solenoid_col_rado $=$

tbench_solenoid_col_radi $+5.433 * \mathrm{~cm}$

230 tbench_solenoid_col_b = Bcoll

1 tbench_solenoid_col_voltage $=0.0$

\#---Drift Spaces ---\#

\# - First Drift

tbench_drift1_zstart $=37 * \mathrm{~cm}$

tbench_drift1_zend $=0.60$

tbench_drift1_ap = machine_piperad

\#-Second Drift

tbench_drift2_zstart $=2.52$

tbench_drift2_zend $=2.67$

tbench_drift2_ap = machine_piperad

\#---Beam size \& position ---\#

beama $0=17.5 \mathrm{e} 0 * \mathrm{~mm}$

beamb0 $=17.5 \mathrm{e} 0 * \mathrm{~mm}$

beamap $0=.0 \mathrm{e} 0 * \mathrm{~mm}$

beambp0 $=.0 \mathrm{e} 0 * \mathrm{~mm}$

beamx $0=.0 \mathrm{e} 0 * \mathrm{~mm}$

beamy $0=.0 \mathrm{e} 0 * \mathrm{~mm}$

beamxp0 $=.0 \mathrm{e} 0 * \mathrm{~mm}$

beamyp0 $=.0 \mathrm{e} 0 * \mathrm{~mm}$

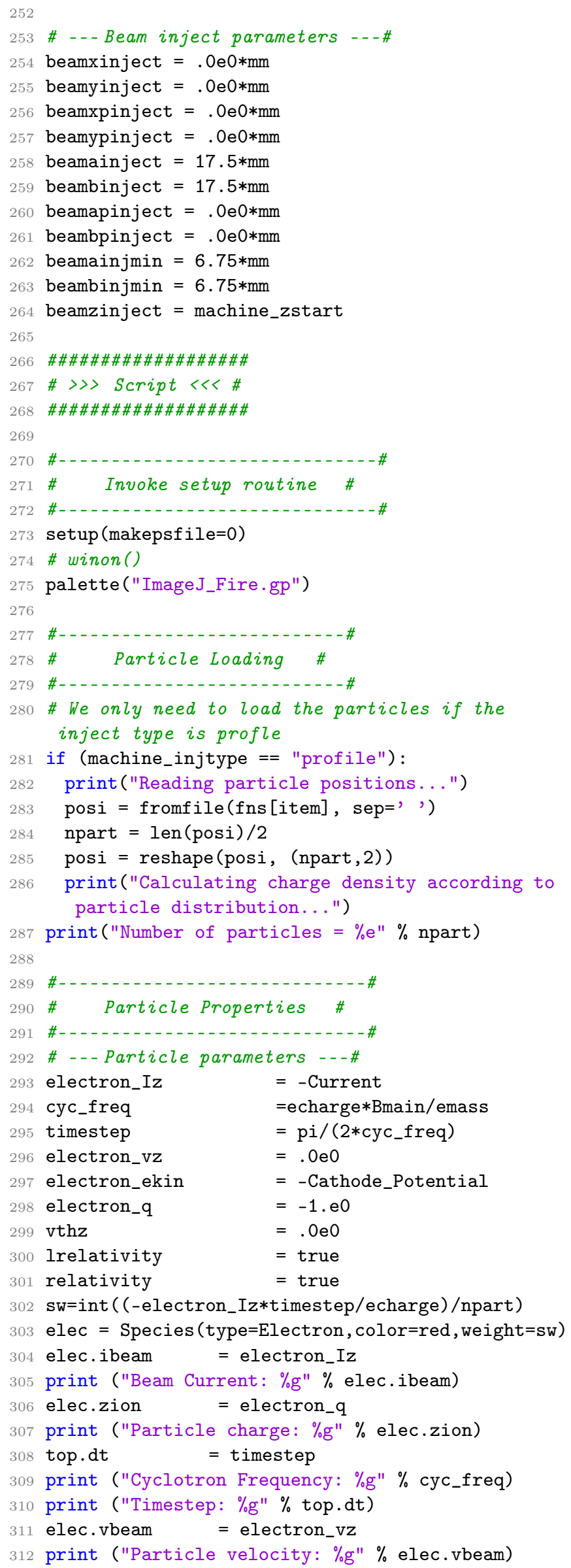




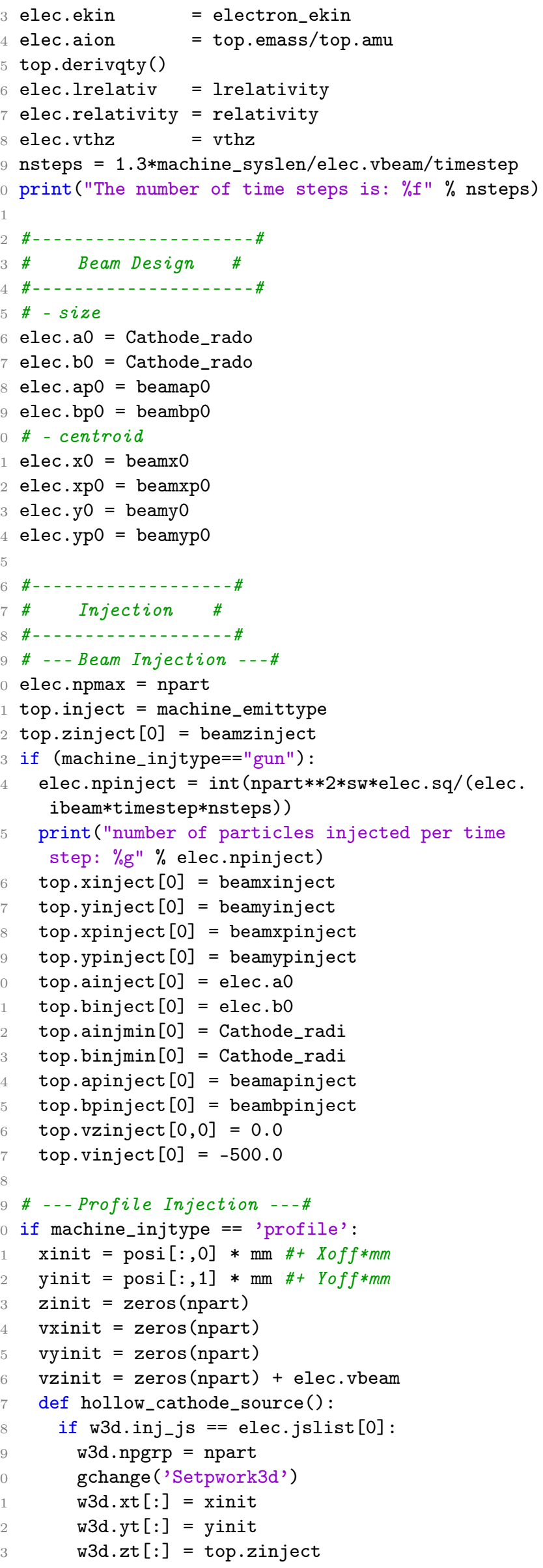

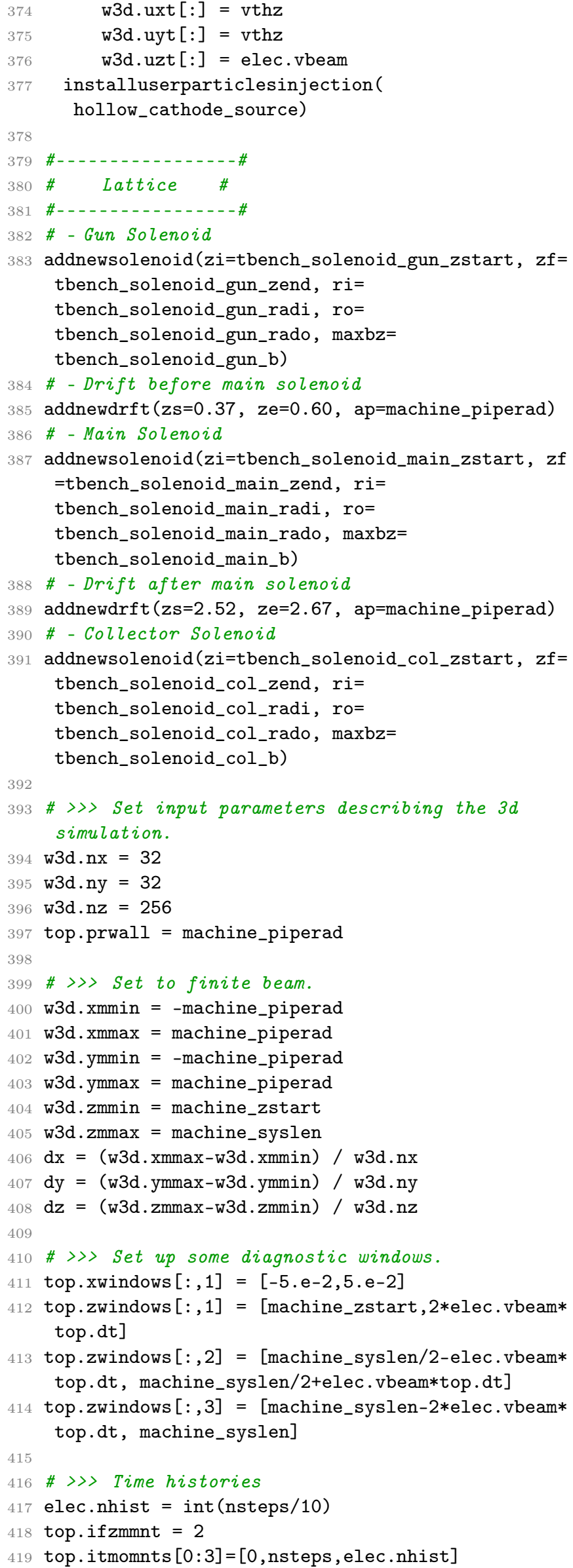




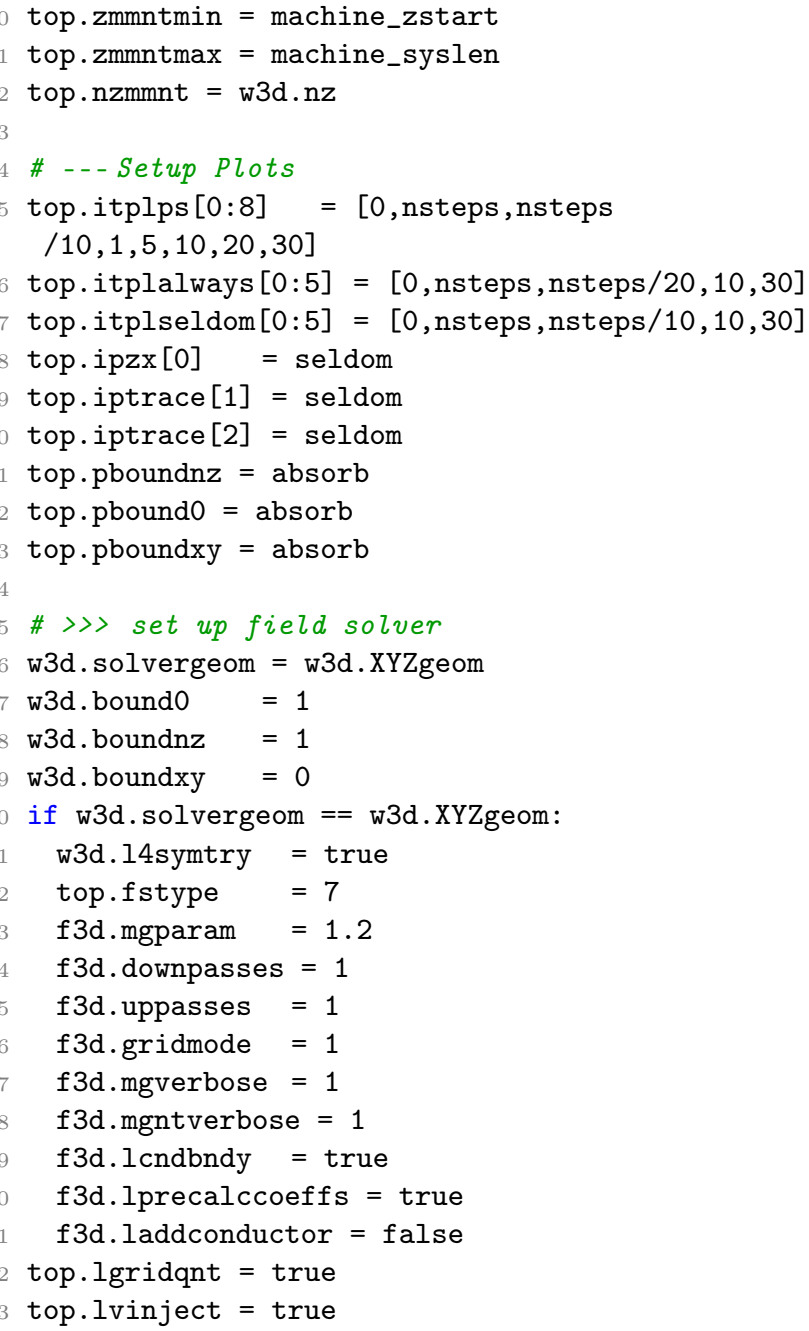




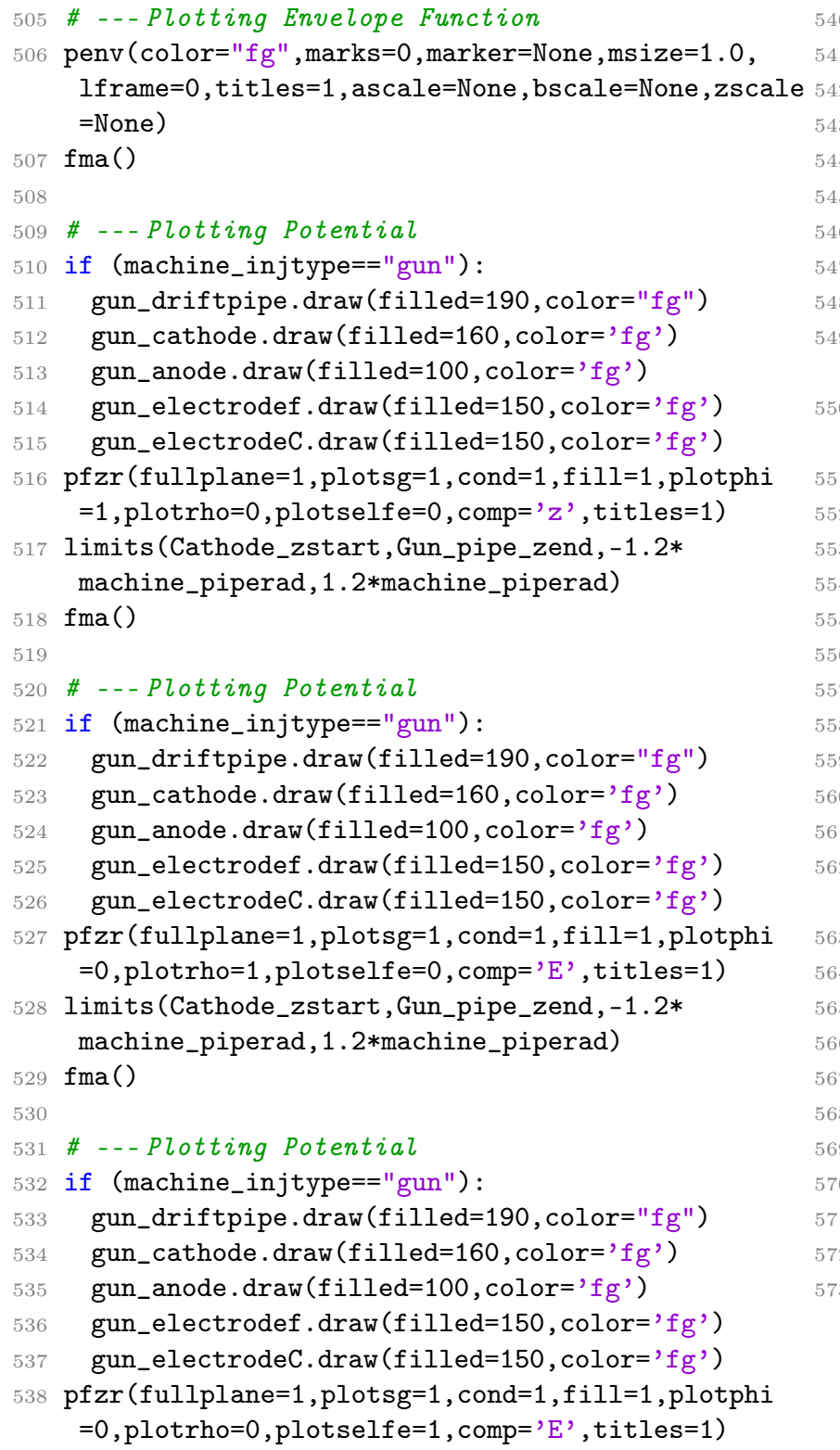

\section{0 fma()}

\#---Plotting Electric Field

if (machine_injtype=="gun"):

gun_driftpipe.draw (filled=190, color $=" f g "$ )

gun_cathode.draw ( $f$ illed $=160$, color $=$ ' $f g$ ')

gun_anode.draw (filled $=100$, color=' $f g$ ')

gun_electrodef .draw (filled $=150$, color $=$ ' $f g$ ')

gun_electrodeC.draw (filled=150, color=' $f g$ ')

9 pfzr (fullplane=1,plotsg=1, cond=1,fill=1,plotphi $=0$, plotrho $=0$, plotselfe $=1$, comp=' $z$ ', titles $=1$ )

550 limits (Cathode_zstart,Gun_pipe_zend, -1.2* machine_piperad,1.2*machine_piperad) machine_injtype+"_"+file_ending+"* . ./Results/"+ date+"/")

\section{Attachments/tbench_complex_app.py}




\section{Appendix D}

\section{Dimensions}

\section{D.1 Electron Guns}

Hollow Electron Gun 1 Inch

\begin{tabular}{|l|r|}
\hline Cathode & {$[\mathrm{mm}]$} \\
\hline Length & 29.25 \\
Inner Radius & 6.75 \\
Outer Radius & 12.7 \\
Radius of Curvature & 10 \\
\hline \hline Anode & {$[\mathrm{mm}]$} \\
\hline Distance to Cathode & 9.48 \\
Length & 85.2 \\
Inner radius & 14.25 \\
Outer Radius & $22 \mathrm{~d}$ \\
Anode radius of Curvature & 3.5 \\
\hline \hline Electrode F & {$[\mathrm{mm}]$} \\
\hline Length & 74 \\
Inner Radius & 13.1 \\
Outer Radius & 15 \\
Radius of Curvature & 1.4 \\
\hline \hline Electrode C & {$[\mathrm{mm}]$} \\
\hline Length & 49.5 \\
Inner Radius & 20.5 \\
Outer Radius & 22 \\
Radius of Curvature & 0.75 \\
\hline
\end{tabular}

\section{Hollow Electron Gun 0.6 Inch}

\begin{tabular}{|l|r|}
\hline Cathode & {$[\mathrm{mm}]$} \\
\hline Length & $\mathrm{e}$ \\
Inner Radius & 9 \\
Outer Radius & 15.24 \\
Radius of Curvature & 10 \\
\hline \hline Anode & {$[\mathrm{mm}]$} \\
\hline Distance to Cathode & 9.48 \\
Length & 90 \\
Aperture & 18 \\
Outer Radius & 40 \\
\hline \hline Electrode F & {$[\mathrm{mm}]$} \\
\hline Length & 38.9 \\
Inner Radius & 8.05 \\
Outer Radius & 11.1 \\
\hline \hline Electrode C & {$[\mathrm{mm}]$} \\
\hline Length & 20.5 \\
Inner Radius & 17.0 \\
Outer Radius & 20.5 \\
\hline
\end{tabular}




\section{D.2 Electron Lenses}

\section{Tevatron Electron Lens 2}

\begin{tabular}{|l|r|}
\hline \hline Bend Solenoids & {$[\mathrm{mm}]$} \\
\hline Width & 90 \\
Inner Radius & 193 \\
Outer Radius & 265 \\
\hline \hline Main Solenoid & {$[\mathrm{mm}]$} \\
\hline Length & 2688.5 \\
Inner Radius & 200 \\
Outer Radius & 344.8 \\
\hline \hline Collector and Gun Solenoid & {$[\mathrm{mm}]$} \\
\hline Length & 500 \\
Inner Radius & 280 \\
Outer Radius & 334.3 \\
\hline \hline Path lengths & {$[\mathrm{mm}]$} \\
\hline Main Solenoid - Nearest Bend & 82.7 \\
Inter Bend Distance & 52.9 \\
Cathode Surface - First Bend & 281.6 \\
\hline \hline
\end{tabular}

\section{Tevatron Electron Lens Test Stand}

\begin{tabular}{|l|r|}
\hline \hline Main Solenoid & {$[\mathrm{mm}]$} \\
\hline Length & 1920 \\
Inner Radius & 100 \\
\hline \hline Collector and Gun Solenoid & {$[\mathrm{mm}]$} \\
\hline Length & 500 \\
Inner Radius & 140 \\
\hline \hline Drift Pipe & {$[\mathrm{mm}]$} \\
Length & 2860 \\
Inner Radius & 30 \\
\hline Path lengths & {$[\mathrm{mm}]$} \\
\hline Main Solenoid - Nearest Bend & 82.7 \\
Inter Bend Distance & 52.9 \\
Cathode Surface - First Bend & 281.6 \\
\hline \hline
\end{tabular}




\section{Appendix E}

\section{Maps}

\section{E.1 Map of LHC Collimators}

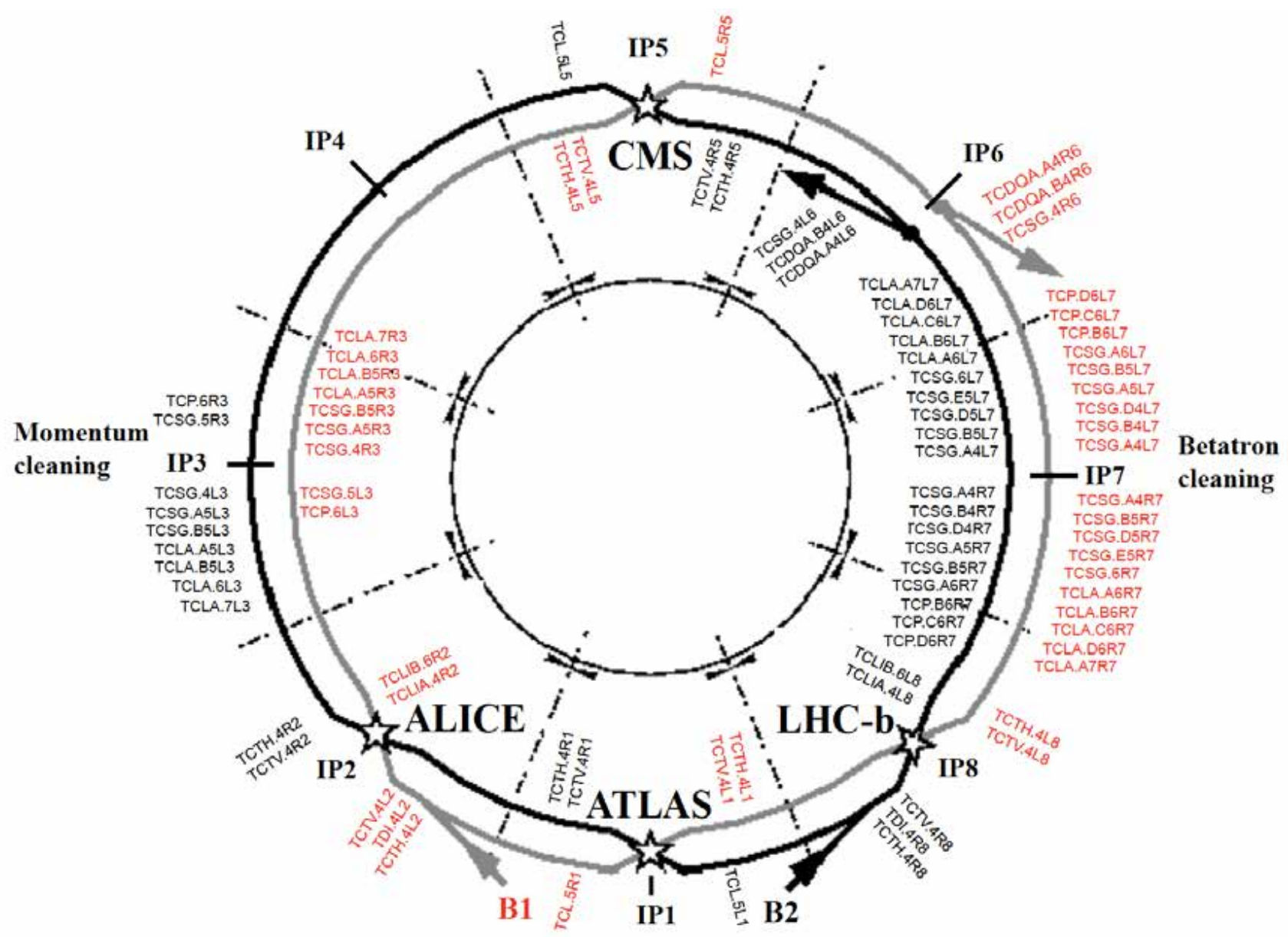

Figure E.1: The super-symmetry of the LHC. IP1, IP2, IP5 and IP8 are the experimental halls ALICE, ATLAS, CMS and LHC-b respectively. IP3 and IPr are the momentum and betatron cleaning sites and IP6 contains the beam dumps. 


\section{E.2 Map of Tevatron}

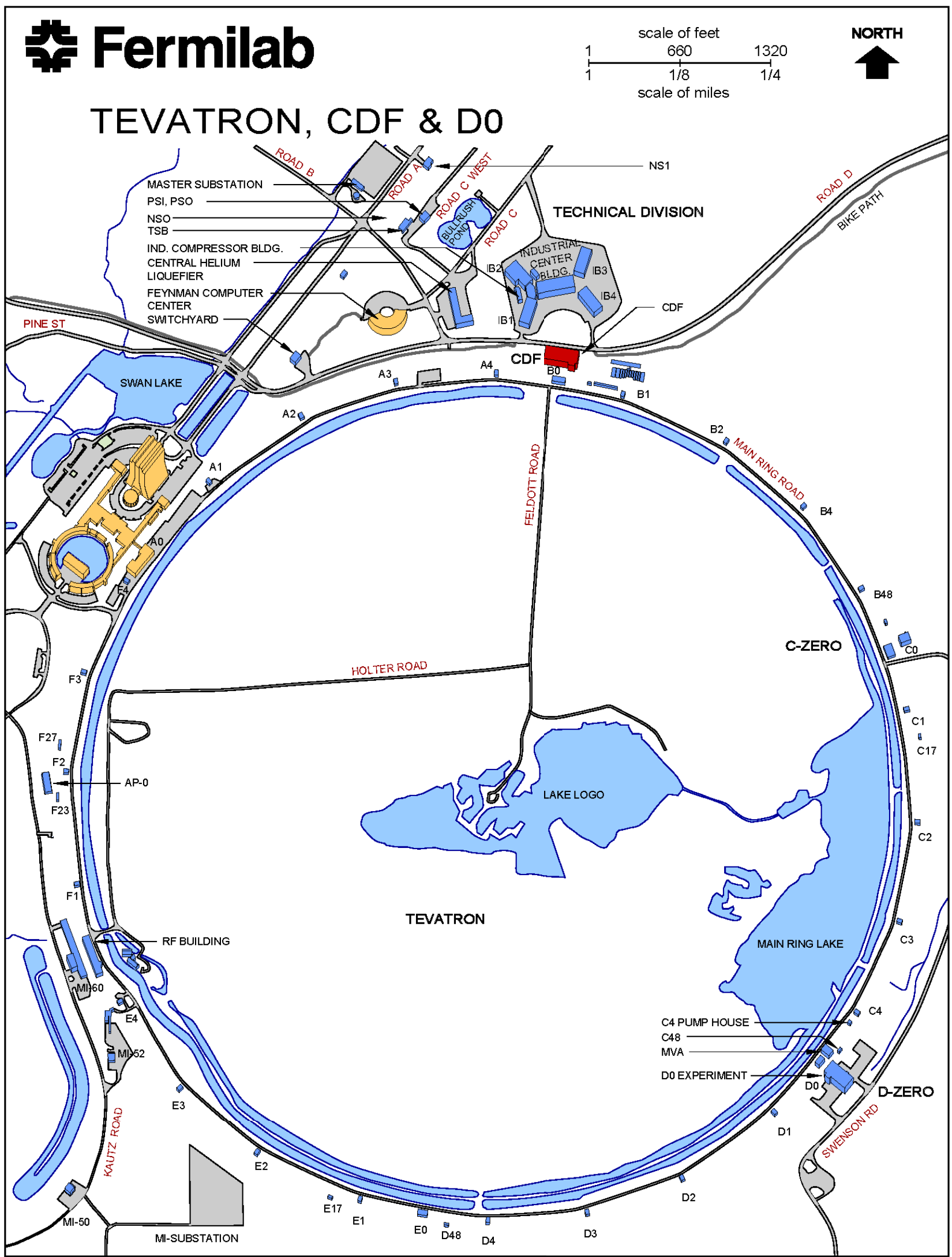

Figure E.2: Map of the tevatron and its various sectors 